\title{
Laser-Induced \\ Subsurface Modification \\ of Silicon Wafers
}

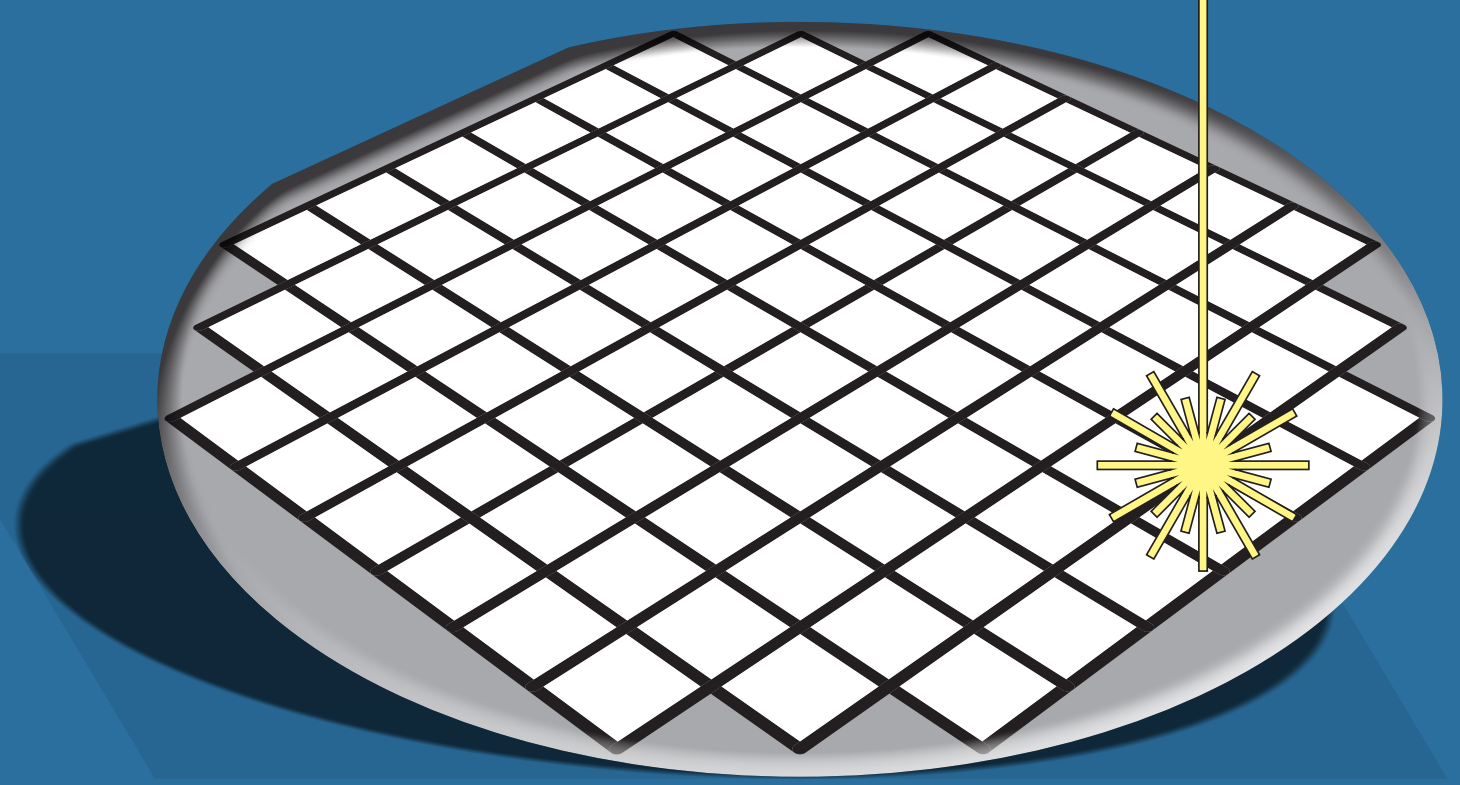

Paul Verburg 



\section{LASER-INDUCED SUBSURFACE MODIFICATION OF SILICON WAFERS}

Paul Christiaan Verburg 


\section{Promotiecommissie:}

Voorzitter en secretaris:

Prof. dr. G.P.M.R. Dewulf

Universiteit Twente

Promotor:

Prof. dr. ir. A.J. Huis in 't Veld

Universiteit Twente

Co-promotor:

Dr. ir. G.R.B.E. Römer

Universiteit Twente

\section{Leden:}

Prof. dr. ir. H.W.M. Hoeijmakers

Universiteit Twente

Prof. dr. J.G.E. Gardeniers

Universiteit Twente

Prof. Dr.-Ing. habil. A. Ostendorf

Ruhr-Universität Bochum

Prof. dr. ir. M.G.D. Geers

Prof. dr. ir. A. de Boer

Technische Universiteit Eindhoven

Universiteit Twente

This work was performed at the Chair of Applied Laser Technology, Group of Mechanical Automation and Mechatronics, Department of Mechanics, Solids, Surfaces \& Systems $\left(\mathrm{MS}^{3}\right)$, Faculty of Engineering Technology, University of Twente, P.O. Box 217, 7500 AE Enschede, The Netherlands.

Financial support was provided by Point-One R\&D project PNE09009 and Advanced Laser Separation International N.V. Point-One is an innovation programme of the Dutch ministry of Economic Affairs.

On the cover: a drawing of a wafer containing multiple identical dies.

Laser-Induced Subsurface Modification of Silicon Wafers

Paul Christiaan Verburg

PhD Thesis, University of Twente, Enschede, The Netherlands.

ISBN 978-90-365-3838-1

DOI 10.3990/1.9789036538381

Copyright (C)2015 P.C. Verburg.

Printed by Gildeprint Drukkerijen, Enschede, The Netherlands. 


\title{
LASER-INDUCED SUBSURFACE MODIFICATION OF SILICON WAFERS
}

\author{
PROEFSCHRIFT
}

ter verkrijging van de graad van doctor aan de Universiteit Twente, op gezag van de rector magnificus, prof. dr. H. Brinksma, volgens besluit van het College voor Promoties in het openbaar te verdedigen op vrijdag 13 maart 2015 om 14:45 uur

door

Paul Christiaan Verburg

geboren op 26 november 1985

te Nijmegen 
Dit proefschrift is goedgekeurd door de promotor:

Prof. dr. ir. A.J. Huis in 't Veld

en de co-promotor:

Dr. ir. G.R.B.E. Römer 


\section{Summary}

One of the unique possibilities that lasers offer is to modify the bulk of transparent materials, without inducing damage to the surface. Applications of subsurface processing are the production of optical components, the fabrication of complex three-dimensional objects and data storage. Most research in this field is targeted towards dielectric materials. The objective of this thesis is to investigate the laser-material interaction during the formation of subsurface modifications in crystalline silicon.

An application of subsurface modifications in silicon is wafer dicing, which is also known as die singulation or die separation. Dicing is the process to separate wafers in individual components called dies. The most common dicing technology is blade dicing, in which a grinding blade is used to separate the dies. This technology requires the use of cooling and lubrication agents during dicing. Moreover, a large amount of debris is generated. Alternatively, laser-induced subsurface modifications can be used to mechanically weaken the wafers, along tracks between the dies. When a force is subsequently exerted on the wafer, it will fracture along the modifications. In this manner, wafers can be separated without loss of material. Moreover, the production of contaminants is minimized.

The main challenge when machining the bulk of silicon is to trigger the absorption of laser energy in a confined volume, at the intended location. A numerical model was developed to gain insight in the laser-material interaction during the production of subsurface modifications in silicon. This model uses a nonlinear Schrödinger equation to simulate the propagation of the laser beam in the material. The response of the material to the absorption of light was modelled by a two-temperature model.

Apart from a numerical model, an experimental set-up was developed to focus laser pulses inside silicon, in order to produce subsurface modifications. Four laser sources were employed for the experimental study, to investigate the effect of various process parameters such as the energy, duration and wavelength of the laser pulse. Based on a comparison of experimental and numerical results, it was found that the numerical model is capable of predicting the required processing conditions for the fabrication of modifications. Moreover, the experimental investigation has shown that the laser modifications are suitable for wafer dicing.

It was concluded that both the temperature-dependence of the linear absorption coefficient and nonlinear absorption can be exploited for the production of modifications, by employing laser sources with wavelengths of 1064 and $1550 \mathrm{~nm}$ respectively. Suitable laser pulses have durations in the short to long nanosecond range, with pulse energies of a few tenths to a few microjoules. These pulses can be generated by common industrial laser sources. 



\section{Samenvatting}

Eén van de unieke mogelijkheden die lasers bieden is het van binnenuit bewerken van transparante materialen, zonder het oppervlak te beschadigen. Toepassingen van bewerkingen onder het oppervlak zijn de productie van optische componenten, de fabricage van complexe driedimensionale objecten en dataopslag. Het meeste onderzoek binnen dit vakgebied is gericht op diëlektrische materialen. De doelstelling van dit proefschrift is om de laser-materiaal interactie tijdens de productie van modificaties onder het oppervlak van kristallijn silicium te onderzoeken.

Een toepassing van modificaties binnenin silicium is het scheiden van wafers in losse onderdelen. De meest gangbare techniek voor het scheiden van wafers maakt gebruik van een roterende slijpschijf. Deze techniek vereist de toepassing van koel- en smeermiddelen tijdens het proces. Bovendien ontstaat een grote hoeveelheid vervuiling. Een alternatief is om met behulp van interne lasermodificaties de wafers mechanisch te verzwakken, langs sporen tussen de onderdelen. Wanneer vervolgens een kracht op de wafer wordt uitgeoefend, breekt deze langs de modificaties. Op deze wijze kan de wafer zonder materiaalverlies gescheiden worden. Bovendien wordt de productie van verontreinigingen tot een minimum beperkt.

De voornaamste uitdaging bij het uitvoeren van laserbewerkingen onder het oppervlak van silicium is om de laserenergie in een begrensd volume te laten absorberen, op de gewenste locatie. Om inzicht te verkrijgen in de laser-materiaal interactie tijdens de productie van modificaties in silicium is een numeriek model ontwikkeld. Dit model maakt gebruik van een niet-lineaire Schrödinger vergelijking om de voortplanting van de laserbundel binnenin het materiaal te simuleren. De reactie van het materiaal op de absorptie van licht is gemodelleerd met een twee-temperaturen model.

Naast een numeriek model is een experimentele opstelling ontwikkeld om laserpulsen binnenin silicium te focusseren, met als doel om modificaties onder het oppervlak te produceren. Vier laserbronnen zijn ingezet voor het experimentele onderzoek, om het effect van verschillende procesparameters zoals de energie, tijdsduur en golflengte van de laserpuls te onderzoeken. Op basis van een vergelijking van numerieke en experimentele resultaten is aangetoond dat het numerieke model in staat is om de vereiste bewerkingscondities voor de fabricage van modificaties te voorspellen. Bovendien is uit het experimentele onderzoek gebleken dat de lasermodificaties geschikt zijn voor het scheiden van wafers.

Er is geconcludeerd dat het mogelijk is om zowel de temperatuursafhankelijkheid van de lineaire absorptiecoëfficiënt als niet-lineaire absorptie in te zetten voor de productie van modificaties, door laserbronnen met golflengtes van respectievelijk 1064 en $1550 \mathrm{~nm}$ te gebruiken. Geschikte laserpulsen hebben een pulsduur in het korte tot lange nanoseconde bereik, met pulsenergieën van een paar tienden tot een paar microjoule. Deze pulsen kunnen gegenereerd worden door gangbare industriële laserbronnen. 



\section{List of publications}

- P. C. Verburg, L. A. Smillie, G. R. B. E. Römer, B. Haberl, J. E. Bradby, J. S. Williams and A. J. Huis in 't Veld. Crystal structure of laser-induced subsurface modifications in Si. Submitted for publication.

- P. C. Verburg, G. R. B. E. Römer, and A. J. Huis in 't Veld. Two-photon-induced internal modification of silicon by erbium-doped fiber laser. Optics Express, 22(18): 21958-21971, 2014.

- P. C. Verburg, G. R. B. E. Römer, and A. J. Huis in 't Veld. Two-temperature model for pulsed-laser-induced subsurface modifications in Si. Applied Physics A: Materials Science \& Processing, 144(4):1135-1143, 2014.

- P. C. Verburg, G. R. B. E. Römer, and A. J. Huis in 't Veld. Processing conditions for laser-induced subsurface modifications in Si. In Proceedings of the 14th International Symposium on Laser Precision Microfabrication, July 23-26, Niigata, Japan, 2013.

- P. C. Verburg, G. R. B. E. Römer, G. H. M. Knippels, J. Betz, and A. J. Huis in 't Veld. Experimental validation of model for pulsed-laser-induced subsurface modifications in Si. In Proceedings of the 13th International Symposium on Laser Precision Microfabrication, June 12-15, Washington DC, USA, 2012.

- L. A. Smillie, P. C. Verburg, J. E. Bradby, and J. S. Williams. Morphology of subsurface modification of Si by nanosecond laser exposure. Presented at the Australian Institute of Physics Congress, December 7-11, Canberra, Australia, 2014. 



\section{Nomenclature}

\section{Mathematical notation}

$\begin{array}{ll}x & \text { Scalar } \\ \mathbf{X} & \text { Matrix } \\ \vec{\bullet} & \text { Vector } \\ \bullet \perp & \text { Transverse } \\ \nabla^{2} \bullet & \text { Laplacian } \\ \nabla \bullet & \text { Gradient } \\ \nabla \cdot \bullet & \text { Divergence } \\ \bullet \bullet \bullet & \text { Inner product } \\ \bullet \times \bullet & \text { Cross product } \\ \mathscr{O}(\bullet) & \text { Order of magnitude }\end{array}$

\section{Abbreviations}

ASE

C-band

CCD

CMOS

$\mathrm{CT}$

DOF

EFD-BPM

FDHM

FEM

FIB

MEMS

MOPA

NA

ND

PBS

SED

SEM

STEM

TEM

WP
Amplified spontaneous emission

Conventional band

Charge-coupled device

Complementary metal-oxide-semiconductor

Computed tomography

Degrees of freedom

Explicit finite difference beam propagation method

Full duration at half maximum

Finite element method

Focussed ion beam

Microelectromechanical systems

Master oscillator power amplifier

Numerical aperture

Neutral density

Polarizing beamsplitter

Selected area diffraction

Scanning electron microscopy

Scanning transmission electron microscopy

Transmission electron microscopy

Waveplate 


\section{Greek symbols}

\begin{tabular}{|c|c|}
\hline$\alpha$ & Linear interband absorption coefficient \\
\hline$\alpha_{\text {exp }}$ & Exponential sampling coefficient \\
\hline$\alpha_{L}$ & Linear thermal expansion coefficient \\
\hline$\beta$ & Two-photon absorption coefficient \\
\hline$\gamma$ & Electron-phonon coupling coefficient \\
\hline$\gamma$ & Shear strain \\
\hline$\gamma_{3}$ & Auger recombination coefficient \\
\hline$\delta$ & Impact ionization coefficient \\
\hline$\epsilon$ & Permittivity \\
\hline$\epsilon$ & Error \\
\hline$\epsilon$ & Normal strain \\
\hline$\vec{\epsilon}$ & Array of strains \\
\hline$\theta$ & Angle with optical axis \\
\hline$\theta$ & Azimuthal coordinate \\
\hline$\lambda$ & Wavelength in a medium \\
\hline$\hat{\lambda}$ & Modified wavelength after beam quality compensation \\
\hline$\mu$ & Mobility \\
\hline$\mu$ & Permeability \\
\hline$v$ & Poisson ratio \\
\hline$v$ & Robert-Asselin filter parameter \\
\hline$\rho$ & Density \\
\hline$\rho$ & Radial coordinate after coordinate transformation \\
\hline$\sigma$ & Free carrier cross-section \\
\hline$\sigma$ & Electrical conductivity \\
\hline$\sigma$ & Standard deviation \\
\hline$\sigma$ & Normal stress \\
\hline$\vec{\sigma}$ & Array of stresses \\
\hline$\tau$ & Relaxation time \\
\hline$\tau$ & Shear stress \\
\hline$\chi$ & Electric susceptibility \\
\hline$\omega$ & Angular frequency \\
\hline$\Omega$ & Domain \\
\hline$\partial \Omega$ & Domain boundary \\
\hline
\end{tabular}

\section{Roman symbols}
A
Component of ray transfer matrix
$B$
Component of ray transfer matrix
$\vec{B}$
Magnetic flux density 
$d$

d

$D$

$\vec{D}$

DFT

$\mathrm{DFT}^{-1}$

e

E

E

$\vec{E}$

\section{E}

$f$

$f$

$f$

$\hat{f}$

F

$g$

$g$

$\hat{g}$

h

h

$\vec{H}$

i

I

j

j

$\hat{j}$

$J$

k

$k$

$k_{B}$

$L$

$L$

$m$

m

Speed of light in a medium

Constant

Specific heat capacity

Capacity

Component of ray transfer matrix

Beam diameter

Depth

Component of ray transfer matrix

Electric flux density

Discrete Fourier transform

Inverse discrete Fourier transform

Elementary charge $=1.602176487 \cdot 10^{-19} \mathrm{C}$

Energy

Young's modulus

Electric field

Constitutive matrix

Focal length

Input function of an integral transform

Component of constitutive matrix

Modified input function of an integral transform

Right hand side terms

Output function of an integral transform

Component of constitutive matrix

Modified output function of an integral transform

Height

Planck's constant $=4.135667516 \cdot 10^{-15} \mathrm{eV} \cdot \mathrm{s}$

Magnetic field

Discrete index

Light intensity

Imaginary unit

Discrete index

Modified Bessel kernel

Bessel function

Wavenumber

Diffusivity/Conductivity

Boltzmann constant $=1.3806504 \cdot 10^{-23} \mathrm{~J} / \mathrm{K}$

Length

Latent heat

Mass

Discrete index 


\begin{tabular}{|c|c|}
\hline$M^{2}$ & Beam quality factor \\
\hline$\vec{M}$ & Magnetization density \\
\hline M & Ray transfer matrix \\
\hline$n$ & Discrete index \\
\hline$n_{2}$ & Kerr coefficient \\
\hline$\vec{n}$ & Normal vector \\
\hline$\tilde{n}$ & Complex refractive index \\
\hline$n$ & Real part of the refractive index \\
\hline$N$ & Carrier density \\
\hline$N$ & Number of samples \\
\hline NA & Numerical aperture \\
\hline$\vec{P}$ & Polarization density \\
\hline$P$ & Power \\
\hline$q$ & Complex Gaussian beam parameter \\
\hline$\vec{q}$ & Flux \\
\hline Q & Source term \\
\hline$r$ & Radial coordinate \\
\hline$\Delta r$ & Discrete step in radial direction \\
\hline$R$ & Surface reflectivity \\
\hline$R$ & Radius \\
\hline$R_{a}$ & Average roughness \\
\hline$t$ & Time \\
\hline$t$ & Duration \\
\hline$\Delta t$ & Discrete step in time \\
\hline$T$ & Temperature \\
\hline$\Delta T$ & Mushy temperature interval to take latent heat into account \\
\hline$u$ & Component of the electric or magnetic field \\
\hline$u$ & Solution of a partial differential equation \\
\hline$\tilde{u}$ & Complex wave envelope \\
\hline$\hat{u}$ & Complex wave envelope after filtering \\
\hline$U$ & Electronic energy \\
\hline$\tilde{U}$ & Complex amplitude \\
\hline$w$ & Beam radius \\
\hline$\Delta \mathrm{wd}$ & Reduction of the working distance to focus inside a material \\
\hline$\vec{W}$ & Electronic heat flux \\
\hline$x$ & Spatial coordinate \\
\hline$\vec{x}$ & Vector containing spatial coordinates \\
\hline$y$ & Spatial coordinate \\
\hline$z$ & Coordinate along the optical axis \\
\hline$\Delta z$ & Discrete step along the optical axis \\
\hline
\end{tabular}




\section{Subscripts}

\begin{tabular}{|c|c|}
\hline$*$ & Effective \\
\hline 0 & Reference/initial value \\
\hline 0 & Vacuum \\
\hline 1,2 & Values before and after a beam propagation step \\
\hline 1,2 & Values before and after a change of the medium \\
\hline$\theta$ & Along the azimuthal coordinate \\
\hline $\mathrm{amb}$ & Ambient \\
\hline aper & Aperture \\
\hline app & Apparent \\
\hline Auger & Auger recombination \\
\hline$b$ & Boiling \\
\hline crit & Critical value \\
\hline C & Conduction band \\
\hline$e$ & Electron \\
\hline$e$ & Electron temperature \\
\hline eff & Effective \\
\hline eq & Equilibrium \\
\hline$f$ & Fluid \\
\hline foc & Focus \\
\hline$g$ & Band gap \\
\hline$h$ & Hole \\
\hline impact & Impact ionization \\
\hline$l$ & Lattice temperature \\
\hline $\operatorname{lin}$ & Linear \\
\hline$m$ & Melting \\
\hline $\max$ & Maximum \\
\hline$N$ & Carrier density \\
\hline$p$ & At constant pressure \\
\hline$p$ & Pulse \\
\hline phot & Photon \\
\hline$r$ & Along the radial axis \\
\hline$s$ & Solid \\
\hline $\operatorname{sim}$ & Simulated \\
\hline real & Physical reality \\
\hline$t$ & Thermal \\
\hline tot & Total \\
\hline trans & Transmitted \\
\hline waist & At the location of the beam waist \\
\hline$z$ & Along the optical axis \\
\hline
\end{tabular}





\section{Contents}

Summary i i

Samenvatting iii

$\begin{array}{ll}\text { List of publications } & \mathbf{v}\end{array}$

Nomenclature vii

\begin{tabular}{lll}
\hline & Introduction & 1
\end{tabular}

1.1 Laser materials processing . . . . . . . . . . . . . . . . . . . 1

1.2 Three-dimensional laser processing of bulk materials . . . . . . . . . . . . 1

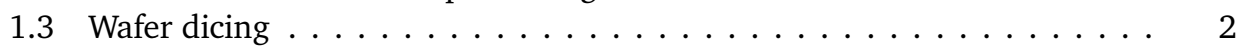

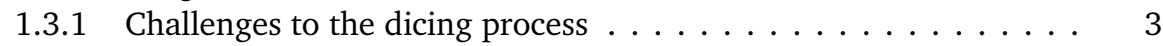

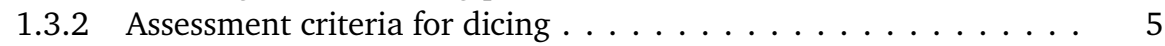

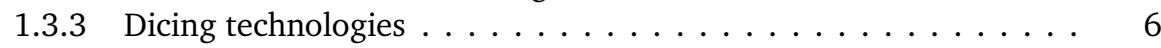

1.4 Laser-induced subsurface separation . . . . . . . . . . . . . . . 8

1.5 Formation of laser-induced subsurface modifications in silicon . . . . . . . 9

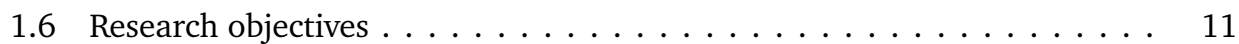

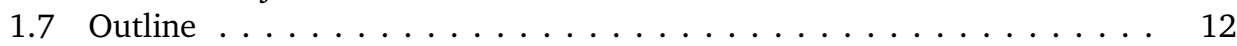

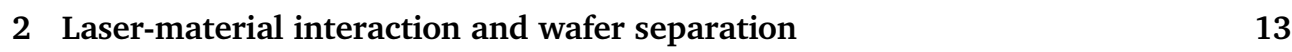

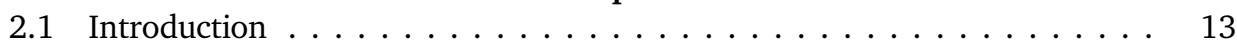

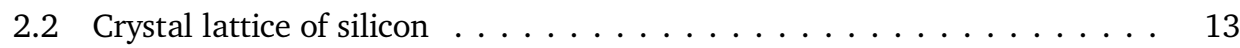

2.3 Laser beam propagation and energy absorption . . . . . . . . . . . . . . . . . 14

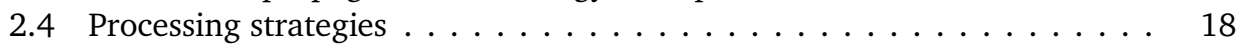

2.5 Time scales of physical phenomena . . . . . . . . . . . . . . . . . . . . . . . . . . . . . . . .

2.6 Modification mechanisms . . . . . . . . . . . . . . . . . . . . . . . . 19

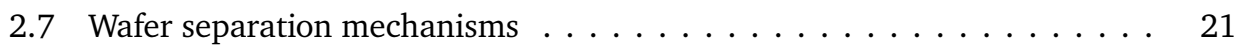

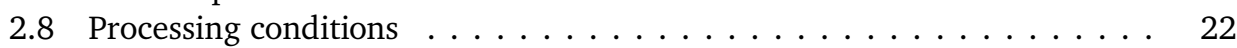

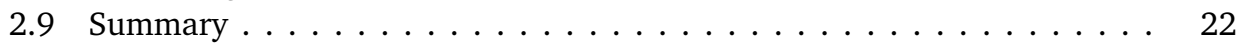

3 Numerical model for laser-material interaction in bulk silicon 25

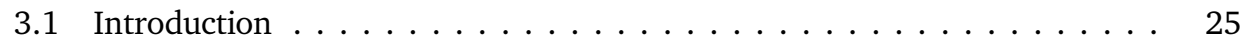

3.2 Two-temperature model . . . . . . . . . . . . . . . . . . . . . . . . . . 25

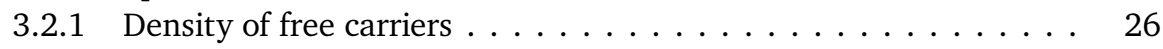

3.2 .2 Lattice temperature . . . . . . . . . . . . . . . . . . . . 26

3.2 .3 Electron temperature . . . . . . . . . . . . . . . . . . . . . 27

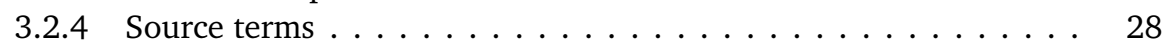

3.2 .5 Mechanical simulations . . . . . . . . . . . . . . . . . . . . . . . . . . 29

3.2 .6 Modification mechanisms . . . . . . . . . . . . . . 30 
3.3 Beam propagation model $\ldots \ldots \ldots \ldots \ldots \ldots \ldots \ldots$

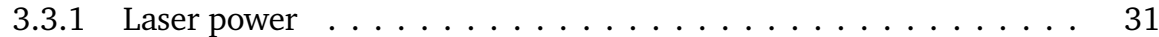

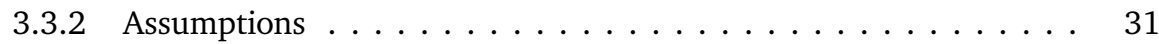

3.3 .3 Maxwell's equations . . . . . . . . . . . . . . . . . . . . . . . 32

3.3 .4 Helmholtz equation . . . . . . . . . . . . . . . . . . . 34

3.3 .5 Paraxial Helmholtz equation $\ldots \ldots \ldots \ldots \ldots \ldots \ldots$

3.3 .6 Nonlinear Schrödinger equation . . . . . . . . . . . . . . . . . . . . . . . . 37

3.3 .7 Collins' diffraction integral . . . . . . . . . . . . . . . . . . . . . . . . . . . . . . . . . .

3.3 .8 Gaussian beams and beam quality . . . . . . . . . . . . . . . . . . . . 39

3.3 .9 Summary of the beam propagation model . . . . . . . . . . . . . . . . 40

3.4 Numerical solvers . . . . . . . . . . . . . . . . . . . . . 41

3.4 .1 Boundary and initial conditions . . . . . . . . . . . . . . 42

3.4 .2 Two-temperature model . . . . . . . . . . . . . . . . . . . . 43

3.4 .3 Time stepping . . . . . . . . . . . . . . . . . . . . . . 44

3.4 .4 Collins' diffraction integral . . . . . . . . . . . . . . . . . . . . . . . 46

3.4 .5 Nonlinear Schrödinger equation . . . . . . . . . . . . . . . . . . 47

3.4.5.1 Finite difference beam propagation methods . . . . . . 47

3.4.5.2 Discretisation of the derivatives . . . . . . . . . . . . 48

3.4.5.3 Crank-Nicolson method . . . . . . . . . . . . . 51

3.4.5.4 Explicit finite difference beam propagation method . . . 52

3.4.5.5 Selection of the finite difference scheme . . . . . . . . 54

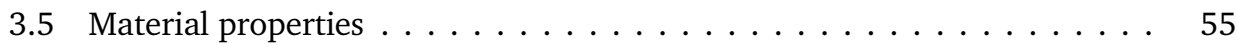

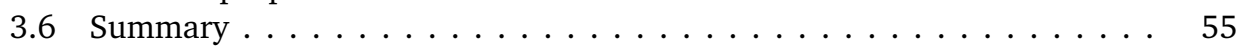

4 Simulation results $\quad 57$

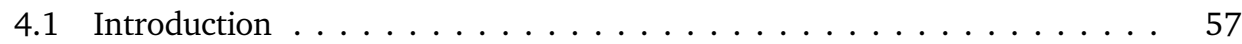

4.2 Laser beam profiles under equilibrium conditions . . . . . . . . . . . . . 58

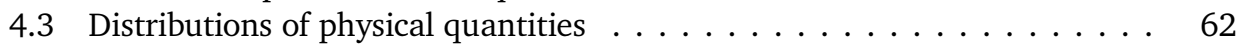

4.3.1 Lattice and electron temperatures . . . . . . . . . . . . . . . 62

4.3 .2 Carrier density . . . . . . . . . . . . . . . . . . . . . . . . 64

4.3 .3 Mechanical stress . . . . . . . . . . . . . . . . . . . . . . . 66

4.3 .4 Laser intensity . . . . . . . . . . . . . . . . . . . . . . . . . . 66

4.4 Modification mechanisms . . . . . . . . . . . . . . . . . . . . . 69

4.5 Parameter studies . . . . . . . . . . . . . . . . . . . . 70

4.5 .1 Parametrisation of the geometry of modifications . . . . . . . . . . . . . . . . . . . 70

4.5 .2 Pulse duration and energy . . . . . . . . . . . . . . . . . . . 71

4.5 .3 Initial temperature $\ldots \ldots \ldots \ldots \ldots \ldots$

4.5 .4 Doping concentration. . . . . . . . . . . . . . . . . . . . . 76

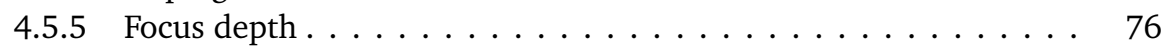

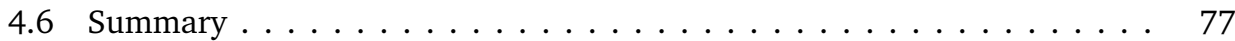

\begin{tabular}{|lll}
5 & Experimental set-up and analysis tools & 79
\end{tabular}

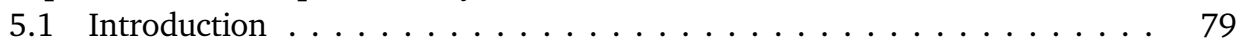

5.2 Laser sources. . . . . . . . . . . . . . . . . . . . . . . . . . . . . . . . . . . . . .

5.3 Laser processing set-up . . . . . . . . . . . . . . . . . 81 


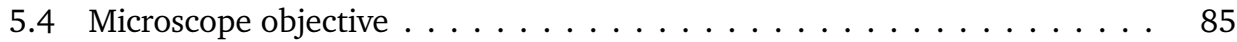

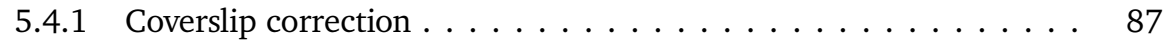

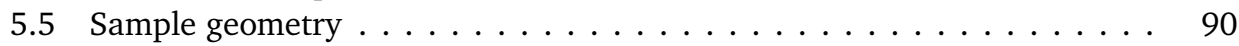

5.6 Non-destructive analysis tools $\ldots \ldots \ldots \ldots \ldots \ldots \ldots \ldots \ldots$. . . . . . 91

5.7 Destructive analysis tools $\ldots \ldots \ldots \ldots \ldots \ldots \ldots \ldots \ldots . \ldots \ldots$

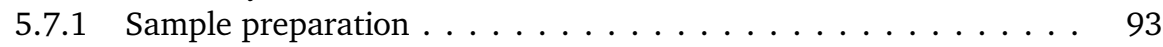

$5.7 .2 \quad$ Surface and lamella analysis tools $\ldots \ldots \ldots \ldots \ldots \ldots \ldots 9$

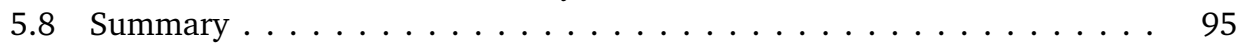

$\begin{array}{lll}6 & \text { Experimental results: geometry } & 97\end{array}$

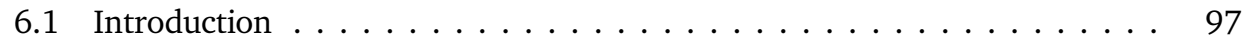

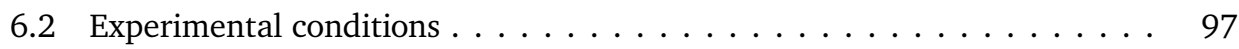

6.3 Model validation for surface damage thresholds $\ldots \ldots \ldots \ldots \ldots \ldots .98$

6.4 Measurement of the modification threshold and geometry . . . . . . . . 99

6.5 Parameter window for the formation of modifications . . . . . . . . . . . 101

6.6 Model validation for subsurface modifications . . . . . . . . . . . . . 104

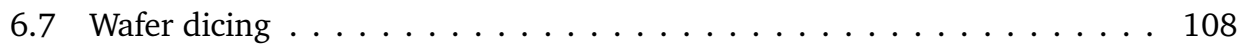

6.8 Modification mechanisms . . . . . . . . . . . . . . . . . . . . . . 112

6.9 Improving the usability of ultra-short pulses $\ldots \ldots \ldots \ldots \ldots \ldots$

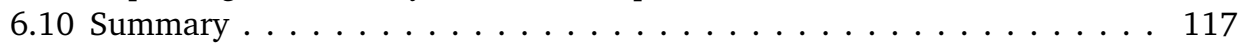

$\begin{array}{lll}7 & \text { Experimental results: crystal structure } & 119\end{array}$

7.1 Introduction . . . . . . . . . . . . . . . . . . . . . . . . 119

7.2 Lamella preparation . . . . . . . . . . . . . . . . . . . . . . . . . 119

7.2.1 Cross-sections along the optical axis . . . . . . . . . . . . 120

7.2.2 Cross-sections perpendicular to the optical axis . . . . . . . . . 122

7.3 Scanning transmission electron microscopy $\ldots \ldots \ldots \ldots \ldots \ldots \ldots$

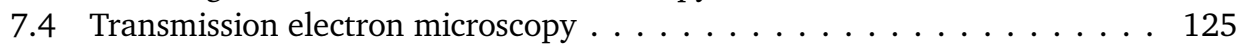

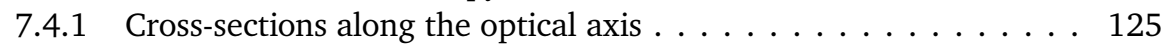

7.4.2 Cross-sections perpendicular to the optical axis . . . . . . . . . 127

7.5 Selected area diffraction $\ldots \ldots \ldots \ldots \ldots \ldots \ldots \ldots \ldots \ldots \ldots$

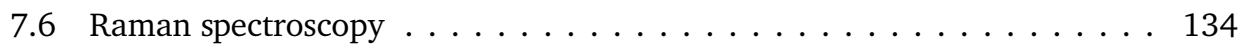

7.7 Modification mechanisms . . . . . . . . . . . . . . . . . . . . . . 135

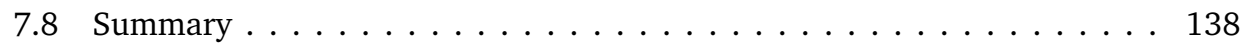

8 Conclusions and recommendations 139

8.1 Conclusions . . . . . . . . . . . . . . . . . . . . . . . . 139

8.2 Recommendations . . . . . . . . . . . . . . . . . . . 141

$\begin{array}{ll}\text { References } & 145\end{array}$

\begin{tabular}{ll}
\hline Acknowledgements & 159
\end{tabular} 



\section{Introduction}

This chapter provides an introduction to the fields of laser materials processing and wafer dicing. Dicing is the process to separate wafers into individual dies. Specific attention is given to the dicing of crystalline silicon wafers using laser-induced subsurface modifications. Based on a review of the existing literature on the formation of subsurface modifications in silicon, the research objectives are established.

\subsection{Laser materials processing}

A laser is a device that emits a coherent beam of light. Laser is an acronym for light amplification by stimulated emission of radiation. After the construction of the first laser in 1960 [1], a vast number of applications have been developed. Optical applications include distance measurements, holography, interferometry, atmospheric measurements, data storage and telecommunications [2].

This work is focussed on the application of lasers for materials processing. Examples of laser materials processing on a macroscopic scale are cutting, drilling, welding, cladding, alloying, surface hardening, marking and rapid prototyping [2, 3]. The application of lasers to process materials on a micrometre scale is referred to as laser micromachining. To machine materials with micrometre resolution, the deposition of laser energy into the material has to be precisely controlled. This requires a well-defined spatial and temporal laser beam profile and precise control over the position of the beam with respect to the sample. Moreover, short laser pulse durations in the nano- to femtosecond range have to be employed, to prevent the laser energy from being conducted away from the volume that is being processed [2]. Applications of laser micromachining are the removal of thin surface layers, surface texturing or the fabrication of micrometre-sized holes by means of ablation [2, 3].

\subsection{Three-dimensional laser processing of bulk materials}

The applications of laser materials processing that are listed in section 1.1 are based on the interaction between laser radiation and material that is located near the surface of a substrate. In this thesis, the formation of laser-induced modifications below the surface is investigated. When processing metals, the optical penetration depth of laser light with optical wavelengths is small, due to strong free carrier absorption. However, semiconductors and dielectric materials show limited absorption of laser light for a range of photon energies below the band gap. For these materials, lasers can be used for 
three-dimensional micromachining, by designing the laser process such that the energy absorption is confined to the bulk of the material [4-10].

So far, most research regarding laser-induced subsurface modifications has been performed on dielectric materials. The most commonly encountered subsurface modifications are decorative markings inside glass [2]. However, there are also many engineering applications of subsurface modifications. A non-exhaustive list of the most important applications is provided in the remainder of this section.

First, applications related to the formation of optical components exist. Many optical applications are based on laser-induced refractive index changes, which can be both positive and negative compared with the unmodified material [4]. A well-known example is the formation of waveguides [11]. Other types of optical components that can be produced are photonic crystals [12], gratings [13], mirrors and beamsplitters [14], lenses [15] and filters [16].

Secondly, subsurface modifications can be employed for three-dimensional data storage [17]. Optical storage media such as CD's, DVD's and Blu-ray discs typically contain a single or a small number of layers to store information. If a similar information density could be obtained along the optical axis of the laser beam as for the transverse directions, a vast increase in storage capacity would be feasible.

Moreover, complex microscopical three-dimensional shapes are fabricated by laserinduced subsurface modifications. The modifications determine the geometry of the shapes by changing the sensitivity of a material to a subsequent chemical etching process. This two-step technology has been used for the production of microfluidic channels [18], rotors [19] and valves [20]. Metal structures may also be produced by laser-induced selective metallization [21]. Additionally, plastic products can be made using two-photon polymerisation, by focussing laser pulses below the surface of a resin [22]. Finally, recent research has shown that patterns of internal modifications improve the strength of glass [23].

Besides engineering applications, confined laser-material interaction may be used to study the behaviour of matter under extreme conditions, by generating pressures in the TPa range as a result of confined microexplosions [8-10, 24]. Such pressures are beyond the hydrostatic stresses that can be induced inside solids by a diamond anvil cell [24].

\subsection{Wafer dicing}

The application of laser-induced subsurface modifications that is studied in this thesis is the dicing of silicon wafers, which is the procedure to separate wafers into individual dies. Wafer dicing is also referred to as die singulation or die separation.

Silicon is the material of choice for the fabrication of integrated circuits. For the production of integrated circuits, high quality monocrystalline silicon is needed, as very small impurities or lattice defects may have a large influence on the performance of the finished products [25]. The Czochralski process [26] is generally used to produce rod-shaped single crystals. The rod that is formed in this manner is cut into disks with standardised dimensions of 1 to 12 inch, called wafers.

A die is a piece of a wafer containing a single product (see figure 1.1). While these products are often integrated circuits, other devices such as optical components or micro- 
electromechanical systems are also produced on wafers. The size of the individual products that are fabricated on a wafer is significantly smaller than the size of the wafer itself. Consequently, many identical components can be fabricated on a single wafer. Hence, a dicing process is needed to separate the components. A single wafer may also contain components with dissimilar dimensions [27]. The lines along which the wafer is cut are referred to as "dicing streets". Wafer dicing is part of the back end processes that include wafer thinning, testing of components, die bonding, wire bonding and packaging [25].

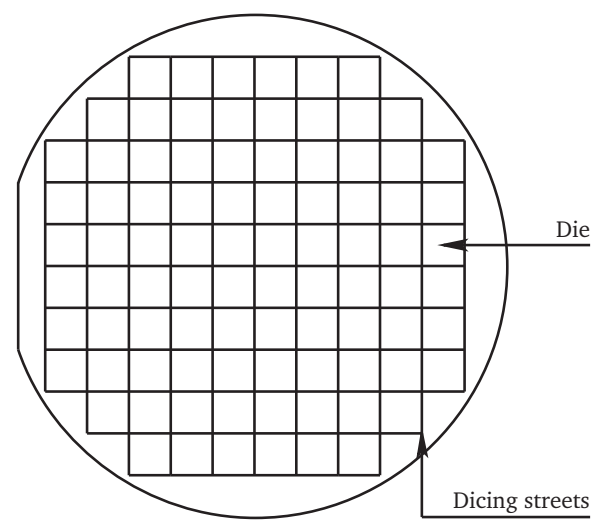

Figure 1.1: Drawing of a wafer containing dies. The tracks along which the wafer should be separated are referred to as "dicing streets".

In this section, an overview is given of the challenges to the wafer dicing process, the characteristics of a dicing process that determine its quality and the different dicing methods that have been proposed.

\subsubsection{Challenges to the dicing process}

There are several developments in the semiconductor industry that present significant challenges to the wafer dicing process. A summary of these challenges is given below.

\section{Low- $\kappa$ layers}

To improve the performance of integrated circuits, layers with a smaller dielectric constant than silicon dioxide (so called low- $\kappa$ layers) are being implemented. These layers present difficulties to the dicing process, due to their poor mechanical properties [27, 28]

\section{Thin wafers}

Ultrathin wafers with a thickness down to $20 \mu \mathrm{m}$ enable chips to be produced that can be integrated in flexible foils [29]. Additionally, thin dies can be stacked to produce three-dimensional integrated circuits $[29,30]$. Dicing wafers with a thickness below $100 \mu \mathrm{m}$, without causing catastrophic damage, is challenging [27]. Additionally, when dicing thin wafers using blade dicing (see section 1.3.3), the lifetime of the blades is negatively impacted as they are unable to self-sharpen [31]. 


\section{Die attach films}

Die attach films are thermoplastic films that are applied to support thin dies during handling or stack dies of varying dimensions [27]. Die attach films complicate the wafer dicing process, since the process has to be capable of dicing through both the wafer and the film [27, 31].

\section{The dicing of small components}

Every dicing process requires a certain street width between the individual components, to ensure that they are not damaged. When producing small components, the dicing streets can occupy a large percentage of the area of the wafer. This space constitutes a loss of potential financial revenue. For certain applications, reducing the width of the dicing streets can improve the productivity by up to 40 percent [32].

\section{The development of microelectromechanical systems}

Microelectromechanical systems (MEMs) consist of a large variety of devices that are produced on wafers, including sensors, mechanical actuators and lab-on-a-chip applications [33]. Besides integrated circuits, MEMs are an important development in the semiconductor industry. As MEMs devices are sensitive to contaminants and are difficult to clean, they require careful treatment during dicing [34]. A special challenge is the dicing of MEMs devices that consist of both silicon and glass [35].

\section{Structures in dicing streets}

Test structures that include metals are commonly placed in dicing streets [27]. Moreover, passivation layers consisting of polymers, oxides or nitrides may be present [31]. Depending on the dicing technology, these structures can affect the dicing process [27, 31].

\section{Three-dimensional die stacking}

The idea of three-dimensionally stacking dies has been proposed [29, 30]. There are several potential advantages of this approach. First, integrated circuits with three-dimensional structures can benefit from reduced latency, because the lengths of communication lines are reduced. For example the communication between logic, memory and interface blocks can be improved. Secondly, one might consider integrating dies that currently need to be packaged in separate chips because they are produced using different technologies, such as microprocessors and random access memory. Finally, three-dimensional stacking allows for improvements in the capabilities of integrated circuits by increasing the number of transistors, without the need to increase the surface area of the dies or to decrease the size of the individual transistors.

For die stacking, the dies should be sufficiently thin to be able to construct through silicon vias with suitable electrical characteristics. Depending on the process choice, the stacking involves complete wafers, individual dies or a combination of both [29, 30]. The advantage of stacking dies instead of complete wafers is that defective components can be excluded before integrating them in a stack.

The dicing of stacks of bonded wafers is challenging for the dicing process [27]. Additionally, when separating dies that need to be included in a stack during a subsequent process step, the geometrical accuracy of the dies and the absence of contamination are important. 


\subsubsection{Assessment criteria for dicing}

For industrial applications, the productivity of a dicing process is defined as the number of wafers that can be handled per hour. For most dicing methods, the throughput depends on the size of the dies, as the die size affects the total length of the dicing streets. However, speed is not the only requirement. Since wafer dicing is part of the back end processes, the production of the active components has already been finished. This means that the wafers represent a large economic value at the dicing stage. Therefore, yield losses due to the dicing process have to be kept to a minimum.

The main requirement for any dicing process is that almost all dies should be properly separated from their neighbours, while keeping the dies intact. Additionally, the dies should satisfy a number of quality criteria. A short overview of the criteria [27] that determine whether the dies can be integrated in finished products is provided below.

\section{Die strength}

The strength of the dies is defined as the amount of stress that they can withstand before they fail. This strength should be high enough such that the dies will not fail during use of the final product. The die strength depends on the edge quality of the separated dies. Defects at the side walls act as crack initiation points, because they induce local stress concentrations when an external force is exerted on the die. As stochastic variations are present in the die strength, it is usually characterised using Weibull statistics [27, 36].

Especially for thin dies, the die strength may be insufficient after the dicing process. Several methods exist to improve the side wall quality and consequently the die strength after dicing, including mechanical or chemical-mechanical polishing and etching.

\section{Chipping}

Chipping is the inadvertent removal of small fragments from the edges of the dies, near the wafer surface. If the size of the fragments exceeds a certain standard, the die has to be rejected. Chipping can affect the strength of a die.

\section{Side wall damage}

Like chipping, damage to the side walls of the dies can influence the die strength. Possible types of damage are deformation, scratching and cracking. Moreover, the material structure in the vicinity of the dicing streets may be altered by stresses or heat input caused by the dicing process.

\section{Kerf geometry}

The geometry of the kerf that remains between the dies after the dicing process is of importance. Damage to the active components should be prevented. Moreover, an incorrect geometry of a die could prevent successful wire bonding or packaging, even if the active component is fully functional.

\section{Surface contamination}

Contamination of the surface of the dies can affect the reliability of the end product. For example, contamination of terminal pads has a negative impact on the wire bonding process. Moreover, electrically conductive contamination may lead to device failure. 


\section{Electrostatic discharge}

The generation of electrostatic charges during dicing has to be prevented as they can induce device failure due to discharge.

\section{Delamination}

When dicing wafers containing low- $\kappa$ layers, delamination at the interface with adjacent materials is an issue, due to the poor adhesion characteristics of such layers. Trenches surrounding the chips that are intended to stop the propagation of cracks may have to be employed to mitigate this problem.

\subsubsection{Dicing technologies}

An overview of the different wafer dicing technologies that have been developed to date is presented below. The most commonly used technology is blade dicing [27]. Wafer dicing by means of laser-induced subsurface modifications, which is the dicing process that is investigated in this thesis, will be introduced in section 1.4 .

\section{Scribe and break}

Scribe and break is the oldest wafer dicing technology [27]. A scribe on the wafer surface can be produced using a diamond tip, a laser or a saw [37]. When bending the wafer around the location of the scribe, the wafer will fracture along the scribe. The ability to dice wafers by the scribe and break process depends on the orientation of the dicing streets with respect to the crystal, as cleavage occurs along one of the preferential cleavage planes.

\section{Blade dicing}

Blade dicing of wafers is a process that employs thin spinning discs with a diamond grit embedded on their edges [27]. Separation takes place by abrasion. An advantage of blade dicing is that it can separate thick wafers and multiple layers in a single pass. There are, however, also a number of drawbacks. Blade dicing generally employs brittle mode fracture that causes chipping and induces subsurface cracks [38]. Moreover, due to the mechanical forces that are exerted on the wafer, catastrophic failure of the wafer can occur when dicing thin wafers with a thickness below $100 \mu \mathrm{m}$ [27]. Additionally, particles are generated that need to be removed. Furthermore, the blades are affected by wear during use and therefore need regular replacement. Finally, blade dicing produces relatively wide dicing streets that cannot be reduced below approximately $30 \mu \mathrm{m}$ [27]. When dicing wafers with small components, this results in a large loss of surface area that could otherwise have been used to produce functional components.

It is possible to induce ductile failure during blade dicing by cutting at low feed rates, which significantly reduces chipping and the formation of microcracks [38]. However, the negative impact on the processing speed will have to be compensated for, e.g. by using gang wheels (parallel blades).

\section{Laser ablation dicing}

Another technique that is used for wafer dicing is ablation by pulsed lasers [27]. To induce ablation, the power of the laser beam should be high enough to cause evaporation or melt ejection. 
One of the main advantages of laser ablation dicing is that it is well suited for dicing thin wafers, as the mechanical forces that are exerted on the wafer are small [27, 39]. This is especially relevant for the dicing of very brittle materials. Additionally, it is possible to cut through layers consisting of different materials, including metals, passivation layers and die attach films [27, 31]. A disadvantage of laser ablation is the difficulty to generate smooth side walls, which negatively affects the die strength [31].

The die strength is related to the pulse duration of the laser source. Picosecond pulses result in a higher die strength compared with a nanosecond process [40]. Also the repeatability of the picosecond process was found to be better, as measured using the Weibull modulus [36, 40]. When comparing femtosecond and picosecond pulses, the shorter pulse duration again showed an improved die strength [41].

\section{Laser microjet separation}

Laser microjet separation is a laser ablation dicing technology that uses a thin water-jet [42]. This water-jet acts as an optical guide for the laser, a coolant and a means of removing the ablated material. Advantages are that contamination is prevented and heat-induced damage is reduced. A disadvantage is that certain devices may be damaged by the water-jet and the particles that it contains.

\section{Thermal laser separation}

Thermal laser separation is a dicing method that is based on the propagation of a crack by thermally-induced stresses [40, 43]. The most extensive version of this process consists of various steps [43]. First, a small defect is produced by a laser or by a mechanical tool. Secondly, stresses are induced by heating the wafer with a continuous wave laser. Finally, a gas or water-based cooling step is performed. A simplified version of the thermal separation process has also been proposed, in which both the production of the initial defect and the crack propagation are performed by a single laser beam, without a cooling step [40].

Advantages of thermal laser separation are a narrow dicing street width, a high edge quality, a high process speed and almost no chipping. However, if water-based cooling is needed, this may damage sensitive devices. Moreover, there are limitations concerning the wafer thickness [43]. Thin wafers may have insufficient thermal mass to generate sufficiently large separation forces. For thick wafers, depending on the laser power, heat conduction is a limiting factor. When employing a laser power of $200 \mathrm{~W}$, separation has been achieved for silicon wafers with a thickness between 50 and $250 \mu \mathrm{m}$ [43].

\section{Hybrid laser/mechanical dicing}

Apart from fully cutting wafers by means of laser ablation, lasers may be employed to produce partial cuts aimed at the removal of specific surface layers [27, 28, 31]. This process is also referred to as grooving [28]. After laser grooving, blade dicing will complete the dicing process. This technology aims to maximize productivity, while preventing damage to low- $\kappa$, passivation or metallic layers.

\section{Plasma dicing}

The dicing of wafers using a plasma has been proposed [27], which is a mask-based process. Therefore, the productivity does not depend on the total length of the dicing streets. Moreover, this process can handle dicing streets that are not straight lines. 


\subsection{Laser-induced subsurface separation}

The application of laser-induced subsurface modifications that is investigated in this thesis, is the dicing of crystalline silicon wafers. The technology to separate wafers using subsurface modifications is known as laser-induced subsurface separation [27]. Several patents on the subsurface separation of wafers have been published [44] 53], with priority dates from 2000 onwards. Nevertheless, there are many aspects of the formation of subsurface modifications in silicon that require further research (see section 1.6).

The subsurface separation method is based on focussing a laser beam inside a wafer, thereby causing local changes to the crystal structure of the semiconductor [54] (see figure 1.2). Then, in a subsequent step, an external force is exerted on the wafer, causing the wafer to separate along the laser modifications. The external force may be applied using tape stretching [55]. Alternatively, laser-induced thermal gradients can be used to introduce stresses, similar to the thermal laser separation process [35].

(a)

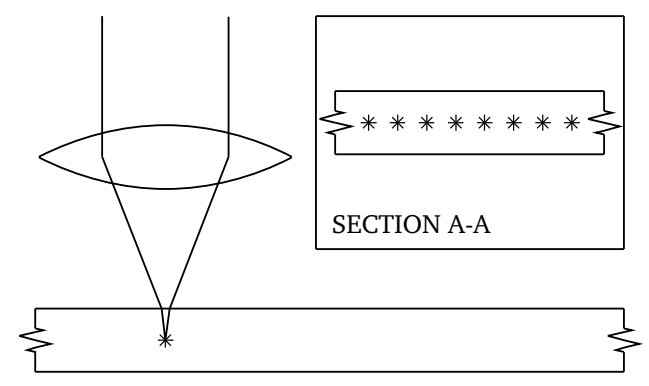

(b)

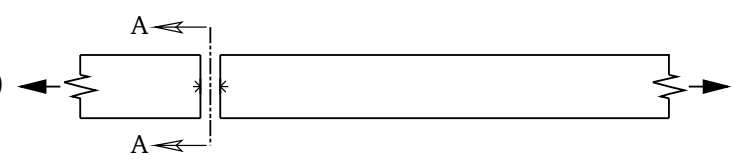

Figure 1.2: Schematic overview of the process to fracture silicon wafers, using pulsed-laser-induced subsurface modifications. (a) Modifications, indicated by asterisks (*), are created below the surface of the wafer. (b) An external force is exerted on the wafer, which causes the wafer to separate along the planes containing laser modifications.

An advantage of subsurface separation over blade dicing or laser ablation dicing is that the kerf width is zero, since no material is removed [31, 55]. Previous research has shown that as close as $10 \mu \mathrm{m}$ to the dicing street, no adverse effects of subsurface modifications in silicon could be found [56]. Moreover, the process is dry and nearly debris-free. Therefore, laser-induced subsurface separation is potentially an ideal technique to separate microelectromechanical devices, as they are sensitive to contamination [35, 57].

A disadvantage is that no opaque layers such as metals are allowed to be present in the path of the laser beam. While this problem might be overcome by irradiating the wafer from the backside, this is not possible in the case of backside metallization. Moreover, depending on the numerical aperture of the focussing objective, a cone has to be free of structures, to allow the laser beam to penetrate into the wafer. This is especially a concern when dicing wafers with a thickness beyond several hundred micrometres, as the radius 
of the cone increases with the distance to the focus of the laser beam. Additionally, the presence of layers of different materials can interfere with the crack propagation during separation [27]. Finally, the manufacturing of semiconductor devices includes doping, which increases the concentration of free carriers and thereby the absorption of laser energy by means of free carrier absorption. Depending on the wafer thickness, the ability of the laser beam to penetrate the wafer becomes an issue when the wafer resistivity is below approximately $0.1 \Omega \cdot \mathrm{cm}$ [58].

\subsection{Formation of laser-induced subsurface modifications in silicon}

The most important step during the subsurface dicing of silicon is the formation of laserinduced subsurface modifications, i.e. the first step in figure 1.2. In section 1.2, several studies regarding the production of subsurface modifications have been cited. However, these are mostly related to the machining of dielectrics. In this section, an overview is provided of the state of the art concerning the bulk processing of silicon by pulsed lasers.

Experiments Compared to the formation of modifications inside dielectrics, limited experimental research has been performed on the production of bulk modifications in silicon. It has been shown that modifications just below the surface can be created, by irradiating silicon samples using $800 \mathrm{~nm}$ femtosecond pulses [59, 60]. However, surface damage was also detected. Moreover, due to strong linear interband absorption, this wavelength cannot be employed to create modifications at a depth of more than tens of micrometres below the surface. Therefore, machining processes that are based on a $800 \mathrm{~nm}$ wavelength will not be considered in this thesis.

An overview of the processing conditions that have been applied during previous experiments concerning the formation of laser-induced subsurface modifications in silicon, using photon energies near and below the band gap, is given in table 1.1. For each experiment, the number of photons that are involved in the interband absorption of laser energy are indicated. Moreover, the outcomes of the experiments are listed.

Since silicon has an indirect band gap, its linear absorption coefficient strongly depends on temperature [67]. Previous work has shown that this characteristic allows for the synthesis of bulk modifications by triggering a thermal runaway, when selecting a wavelength of $1064 \mathrm{~nm}$ that corresponds to a photon energy near the band gap [35, 45, 54, 61]. However, the use of linear absorption results in the absorption of laser energy throughout the path of the laser beam inside silicon, resulting in a limited selectivity.

Several authors investigated the use of two- or three-photon absorption, by employing femtosecond pulses with wavelengths in the range of 1.2 to $2.4 \mu \mathrm{m}$ [62 66 . In these studies, linear interband absorption was prevented by selecting a photon energy well below the band gap. As multiphoton absorption depends on a power function of the light intensity, the laser energy absorption at low intensities is negligible, while strong absorption occurs at high intensities. This allows for selective three-dimensional machining of bulk materials, making it the technology of choice for the subsurface processing of dielectrics [4]. For 
Table 1.1: Overview of processing conditions that were previously investigated for the formation of laser-induced subsurface modifications in crystalline silicon, including the outcomes of the experiments. The numbers of photons correspond to the photons involved in the interband absorption of laser energy. NA is an abbreviation for numerical aperture. A dash indicates that the value was not specified.

\begin{tabular}{lcrrlll}
\hline Wavelength & Photons & $\begin{array}{r}\text { Pulse } \\
\text { duration }\end{array}$ & $\begin{array}{r}\text { Pulse } \\
\text { energy }\end{array}$ & NA & $\begin{array}{l}\text { Location of laser } \\
\text { modifications }\end{array}$ & Ref. \\
\hline $1064 \mathrm{~nm}$ & $1-2$ & $150 \mathrm{~ns}$ & $4 \mu \mathrm{J}$ & - & Subsurface (focus) & {$[61]$} \\
$1064 \mathrm{~nm}$ & $1-2$ & $30 \mathrm{~ns}$ & $20 \mu \mathrm{J}$ & 0.55 & Subsurface (focus) & {$[45]$} \\
$1064 \mathrm{~nm}$ & $1-2$ & $10 \mathrm{~ns}$ & $0.5-12 \mu \mathrm{J}$ & 0.7 & Subsurface (focus) & {$[35]$} \\
$1200 \mathrm{~nm}$ & $1-2$ & $250 \mathrm{fs}$ & $<90 \mu \mathrm{J}$ & 0.2 & No modifications & {$[62]$} \\
$1300 \mathrm{~nm}$ & 2 & $100 \mathrm{fs}$ & $<730 \mathrm{~nJ}$ & 0.3 & No modifications & {$[63]$} \\
$1550 \mathrm{~nm}$ & 2 & $800 \mathrm{fs}$ & $50 \mu \mathrm{J}$ & 1.25 & Surface/subsurface & {$[64]$} \\
$1552 \mathrm{~nm}$ & 2 & $900 \mathrm{fs}$ & $4 \mathrm{uJ}$ & 0.85 & Near back surface & {$[65]$} \\
$2400 \mathrm{~nm}$ & 3 & $70 \mathrm{fs}$ & $1.7 \mu \mathrm{J}$ & 0.5 & Near front surface & {$[66]$} \\
\hline
\end{tabular}

silicon, attempts to induce subsurface damage using multiphoton absorption have been unsuccessful in creating modifications that coincide with the focus of the laser beam (see table 1.1). Subsurface waveguides just below an oxide overcoat have been created by three-photon absorption [66]. However, the modifications could not be created deeper in the bulk of the material. Additionally, a combination of surface and subsurface damage was reported when focussing femtosecond pulses with a wavelength of $1550 \mathrm{~nm}$ inside silicon [64], while performing line scans at a relatively high pulse repetition frequency. The exact depth of the subsurface damage was not identified during this study. Similar experiments showed that subsurface damage could be created, but only for focus positions near the back surface of the wafer [65]. Other studies reported that no modifications were found, when applying single or multiple femtosecond pulses to the same location inside crystalline silicon wafers [62, 63], while using a low pulse repetition frequency to prevent accumulation effects. In spite of the issues that were encountered during previous experiments, there is a strong interest in the development of a multiphoton subsurface modification method for silicon. Four out of the five studies listed in table 1.1 that are based on multiphoton absorption, date from the period between 2012 and 2014.

A major difficulty when experimentally investigating subsurface modifications is their analysis after laser processing, as the modifications are contained in a large volume of unmodified material. Consequently, limited data is available on the geometry and crystal structure of subsurface modifications in silicon. Raman spectroscopy measurements have shown that transformations to amorphous and polycrystalline silicon occur [65].

Numerical modelling Numerical models have previously been published to simulate the lattice temperature during subsurface processing of silicon, based on shape-invariant 
propagation of the laser beam and linear absorption of laser energy [54, 68]. However, other relevant phenomena such as multiphoton absorption, free carrier absorption and physical optics were not considered.

A model for $1300 \mathrm{~nm}$ femtosecond laser pulses focussed inside silicon has been published [69], which simulates the generation of free carriers in detail, by combining a two-temperature model with the finite difference time domain method [70]. However, this model is aimed at simulating the dynamics during a pulse duration of only a hundred femtoseconds. Therefore, effects such as elevated lattice temperatures and the recombination of electron-hole pairs were neglected. Moreover, the finite difference time domain method does not scale well to long pulse durations, as the maximum time step is limited by a stability criterion [70]. Since experimental successes regarding the formation of subsurface modifications in silicon, which coincide with the focus of the laser beam, have so far been achieved using nanosecond pulses (see table 1.1), a numerical model has to be capable of accurately and efficiently simulating these conditions as well.

Another recent model considers the generation of carriers by two-photon absorption and the resulting subsurface refractive index gradients, with the aim to simulate the beam propagation inside silicon [71]. However, neither the electron nor the lattice temperature was included.

Compared with the modelling of the formation of subsurface modifications, models for surface modifications are well-developed. One-dimensional numerical models have been successfully used to quantitatively predict surface damage thresholds of silicon [72-76].

\subsection{Research objectives}

The primary objective of this thesis is to investigate the laser-material interaction during the formation of subsurface modifications in crystalline silicon.

When considering the literature on the formation of subsurface modifications in silicon (see section 1.5), the main gaps in the available knowledge are: (1) a multiphoton subsurface modification method, which performs satisfactorily, has not yet been developed for silicon, (2) a model to quantitatively predict the outcome of the subsurface modification process is not available, (3) the parameter window of the process, including the physics that govern its boundaries, has not yet been established and (4) limited knowledge is available about the crystal structure of subsurface modifications.

To address the issues stated above, the primary objective has been divided in a number of specific goals. These can be categorised in targets concerning the development of the laser process, the numerical model, the experimental investigation and wafer dicing. Regarding the laser process, the objectives are:

- To investigate the suitability of ultra-short pico- to femtosecond pulses for the formation of subsurface modifications in crystalline silicon.

- To develop a multiphoton subsurface modification method for crystalline silicon, which is capable of producing modifications near the focus of the laser beam.

The objective for the model development is: 
- To develop a numerical model to simulate the laser-material interaction inside crystalline silicon that is sufficiently accurate to select suitable laser processing conditions.

For the purpose of validating the numerical model and to obtain detailed information regarding the phase transformations that have occurred, two additional goals concerning the experimental investigation have been established:

- To develop an experimental set-up to produce subsurface modifications in crystalline silicon using different processing conditions.

- To develop analysis methods to identify the geometry and crystal structure of subsurface modifications in crystalline silicon.

Finally, the following objective related to the suitability of the laser-induced modifications for wafer dicing has been defined:

- To assess the suitability of subsurface modifications, obtained using different processing conditions, for the dicing of crystalline silicon wafers.

\subsection{Outline}

This thesis is divided in eight chapters. Chapter 2 provides a description of the laser-material interaction inside bulk silicon, including the relevant physical phenomena and the time scales on which they occur. Moreover, the wafer separation mechanisms are discussed and processing strategies are proposed that are potentially suitable for the subsurface modification of silicon.

Chapter 3 describes the numerical model that was developed to simulate the lasermaterial interaction during the formation of laser-induced subsurface modifications in silicon. The equations to model the response of the material to the laser energy absorption and the laser beam propagation will be discussed. Additionally, the numerical solvers are described.

Chapter 4 contains the results of the parameter studies that were performed using the numerical model. The parameters that were investigated are the wavelength, pulse duration, pulse energy, initial temperature, focus depth, beam profile and doping concentration.

Chapter 5 describes the experimental set-up that was designed to produce subsurface modifications under various processing conditions. Moreover, the sample analysis techniques are presented. The purpose of the experiments is threefold. That is, to validate the numerical results, to study the phase transformations that have taken place and to assess the suitability of the modifications for wafer dicing.

The experimental results are split between two chapters: chapter 6 describes the geometry of the subsurface modifications and their suitability for wafer dicing while chapter 7 is focussed on the identification of their crystal structure.

Finally, the conclusions and recommendations can be found in chapter 8 


\section{Laser-material interaction and wafer separation}

In this chapter, descriptions of the physical phenomena that occur during the interaction of a laser beam with bulk crystalline silicon are provided. Based on these phenomena, strategies to selectively absorb laser energy inside silicon are established.

\subsection{Introduction}

To accomplish the research objectives that were stated in section 1.6 , numerical simulations and experiments regarding the formation of laser-induced modifications inside crystalline silicon were carried out. To select the physics that should be included in the numerical model and to determine which processing conditions are worthwhile to investigate, an overview of the relevant physical phenomena and their characteristic time scales is required.

In the following sections, the underlying physics of the laser-material interaction inside silicon will be described, including the laser energy absorption, the mechanisms that induce permanent changes to the material structure and the optics that govern the laser beam propagation. Additionally, the role of the laser modifications during wafer separation is discussed. Special attention is given to the mechanisms of laser energy absorption that allow the absorption to be limited to a small subsurface volume, as this is one of the most important requirements for the formation of subsurface modifications in silicon. Based on these mechanisms and the availability of laser sources, the laser wavelengths that will be considered in this thesis are selected.

\subsection{Crystal lattice of silicon}

Diamond cubic silicon is a semiconductor. Semiconductors are materials with an electrical resistivity between $10^{-2}$ and $10^{9} \Omega \cdot \mathrm{cm}$ at room temperature [77]. They are characterised by a band gap, a range of electron energy states between the valence and conduction bands that cannot be occupied. When sufficient energy is supplied to an electron in the valence band, it transfers to the conduction band. Both the hole that remains in the valence band, and the excited electron itself, increase the conductivity of the semiconductor [77].

Under conditions of room temperature and atmospheric pressure, the stable crystalline phase of silicon is diamond cubic [77], which is also referred to as Si-I [78] (see figure 2.1). Silicon can also exist in amorphous phases with various densities [79]. In the current work, a modification is defined as any change to the material compared with the original almost defect-free diamond cubic silicon crystal.

Besides Si-I, other crystalline phases of silicon exist. Due to the high pressures that might be induced when focussing a laser beam inside bulk silicon, high-pressure phases could be generated. In table 2.1, the different known phases of silicon are listed, including the 


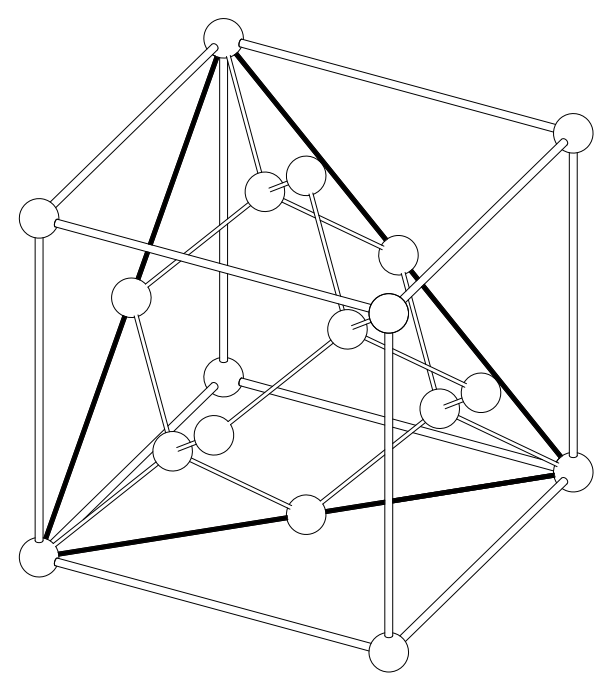

Figure 2.1: Unit cell of Si-I, the locations of atoms in the unit cell were obtained from reference [77]. The top and bottom of the cell are aligned with the surfaces of $<100>$ wafers. The $\{111\}$ plane is indicated using thick lines.

pressure and temperature ranges in which they exist. When the highest pressure level is listed first, the corresponding phase is a metastable phase that is formed during unloading from that pressure level. For some phases, large discrepancies exist between data obtained from different sources.

Si-XII and Si-III are formed from Si-II during unloading [78]. Therefore, at least a pressure of $8.8 \mathrm{GPa}$, corresponding to the start of the formation of Si-II, has to be overcome to form any phase other than Si-I. While the data in table 2.1, based on reference [78], indicate that $\mathrm{Si}$-XII does not exist at pressures below $2 \mathrm{GPa}$, a more recent study has shown that Si-XII persists to atmospheric pressure, albeit in a low concentration [80]. Si-IV is formed by heating of Si-III [78] or by inducing large shear stresses in Si-I, at temperatures of approximately 700 to $1000 \mathrm{~K}$ [81].

\subsection{Laser beam propagation and energy absorption}

The formation of laser-induced subsurface modifications inside crystalline silicon depends on the localised absorption of laser energy in the bulk of the material. The most important physical quantity that governs the absorption of laser energy is the distribution of the light intensity inside silicon. To establish what intensities occur inside silicon, the power density profile of the laser beam as it propagates through the material has to be determined. The most general description of the propagation of a laser beam, within the field of classical electrodynamics, is given by Maxwell's equations [85]. In section 3.3, the beam propagation model that was applied during the numerical simulations will be discussed, including the approximations compared with a full solution of Maxwell's equations. In addition to the 
Table 2.1: Overview of the known crystalline phases of silicon. Unless mentioned otherwise, the data were obtained from reference [78].

\begin{tabular}{llll}
\hline Phase & Stability & Structure & Pressure range (GPa) \\
\hline Si-I & Stable & Diamond cubic & $0-12.5$ \\
Si-II & Stable & Body centred tetragonal & $8.8-16$ \\
Si-III & Metastable & Body centred cubic & $2.1-0$ \\
Si-IV & Metastable & Diamond hexagonal & - \\
Si-V & Stable & Primitive hexagonal & $14-35$ \\
Si-VI & Stable & Orthorhombic [82] & $34-40$ \\
Si-VII & Stable & Hexagonal close packaged & $40-78.3$ \\
Si-VIII & Metastable & Tetragonal & $14.8-0$ \\
Si-IX & Metastable & Tetragonal & $12-0$ \\
Si-X & Stable & Face centred cubic & $78.3-230$ \\
Si-XI & Stable & Body centred orthorhombic & $13-15$ \\
Si-XII & Metastable & Trigonal (rhombohedral) & $12-2.0$ \\
Si-XIII [83] & Metastable & Unknown & Heating of Si-III or Si-XII. \\
\hline [84] & - & Possibly body centred cubic & $>250$ \\
\hline
\end{tabular}

wave-like behaviour of light as described by Maxwell's equations, it has been shown that light also exhibits a particle-like behaviour [86]. These particles, which contain a quantized amount of energy that depends on the wavelength of the light in vacuum, are known as photons. For the laser-processing of semiconductors, the ratio between the photon energy and the band gap of the semiconductor is of great importance. Consequently, depending on the physical phenomenon that is being considered, the wave or particle description of light has to be applied.

Changes to the refractive index The power density profile, of a laser beam inside silicon, changes during the laser pulse. Apart from the absorption of energy, the beam profile is affected by gradients in the refractive index. A positive change to the refractive index may be induced by:

- An increase in the lattice temperature [87].

- The optical Kerr effect, i.e. the dependence of the refractive index on the light intensity [88].

The Kerr effect results in self-focussing above the following critical power level [10]:

$$
P_{\text {crit }}=\frac{\lambda_{0}^{2}}{2 \pi n_{0} n_{2}},
$$


where $\lambda_{0}$ is the wavelength of the laser light in vacuum. The refractive index $n$ is related to $n_{0}$ and $n_{2}$ by $n=n_{0}+n_{2} I$, with $I$ the laser intensity. Equation (2.1) is an approximation, as the actual critical power depends on the beam profile [89]. Exceeding this power level results in delocalization of the laser energy due to self-focussing and filamentation and should therefore be avoided. For wavelengths up to $6 \mu \mathrm{m}$, the Kerr coefficient $n_{2}$ of silicon remains relatively constant with a strong peak around $1.9 \mu \mathrm{m}$ [90]. A decrease in the refractive index is caused by:

- An increase in the density of electron-hole pairs [91]. Electron-hole pairs are generated by interband absorption of photons, resulting in the promotion of electrons from the valence to the conduction band. Additionally, impact ionization, which is a process that uses energy from free carriers to generate additional carriers [92], may increase the density of free carriers.

Since the refractive index is simultaneously affected by multiple phenomena during the internal processing of silicon, complex refractive index distributions may arise. How these patterns affect the light intensity distribution inside silicon will be evaluated using numerical simulations (see chapter 4).

Laser energy absorption To produce subsurface modifications in any material, the laser energy has to be absorbed in a confined volume below the surface. This implies that the material has to be sufficiently transparent for a laser beam of a certain wavelength to reach the bulk of the material. This requirement is in direct competition with the necessity of effective absorption of laser energy in the volume that is to be modified. Therefore, a processing strategy has to be established that results in the silicon behaving opaque or transparent, depending on the location in the volume. Absorption and refraction of light are not independent phenomena. The wavelength-dependent absorptivity and refractive index are inherently linked by the Kramers-Kronig relations [85].

The ratio between the energy of the photons and the band gap determines which mechanisms of laser energy absorption are relevant. For crystalline silicon, the band gap energy $E_{g}$ is $1.11 \mathrm{eV}$ at a temperature of $300 \mathrm{~K}$ [77]. The wavelength in vacuum which corresponds to this energy is given by the Planck relation as [85]

$$
\lambda_{0}=\frac{h c_{0}}{E_{g}}=\frac{4.1357 \cdot 10^{-15} \mathrm{eV} \cdot \mathrm{s} 2.9979 \cdot 10^{8} \mathrm{~m} / \mathrm{s}}{1.11 \mathrm{eV}} \approx 1.1 \cdot 10^{-6} \mathrm{~m}=1.1 \mu \mathrm{m},
$$

in which $h$ is Planck's constant and $c_{0}$ the speed of light in vacuum. An overview of the different mechanisms of laser energy absorption in silicon is given below.

\section{Linear interband absorption}

Linear interband absorption is the excitation of an electron, from the valence to the conduction band, by the absorption of a single photon. To enable linear absorption, the energy of the photons should be close to or exceed the band gap energy. Because silicon has an indirect band gap, not only photons and electrons are involved in the linear absorption process. The absorption or generation of a phonon, which is a quantum description of lattice vibrations, is also required [77]. 
Since the band gap of silicon is a function of temperature [93] and because phonons are involved in the absorption of light, the linear absorption coefficient strongly depends on temperature for photon energies near the band gap (see figure 2.2).

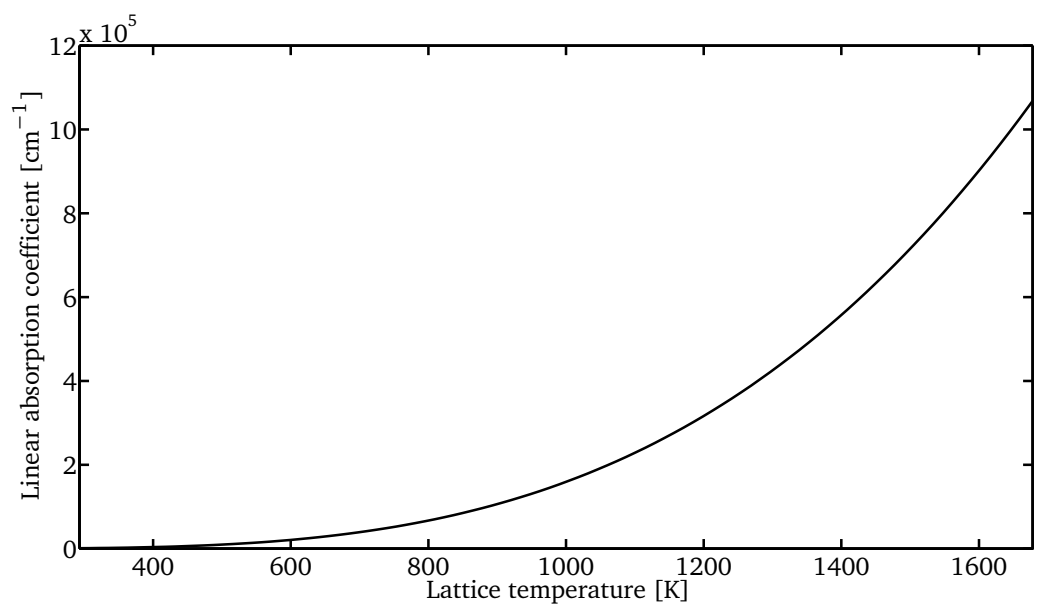

Figure 2.2: Linear absorption coefficient of diamond cubic silicon as a function of the lattice temperature, for a wavelength of $1064 \mathrm{~nm}$. The temperature ranges from room temperature to the melting point. The data were computed based on reference [67].

\section{Multiphoton interband absorption}

Apart from linear absorption, an electron may transfer from the valence to the conduction band by absorbing the combined energy of two or more photons [7]. The multiphoton ionization rate is described by a power function, where the power is given by the number of photons that are involved in the absorption process. Consequently, multiphoton absorption is a nonlinear phenomenon.

\section{Free carrier absorption}

Free carriers contribute to the absorption of laser energy. A certain concentration of carriers is always present depending on the temperature [77]. During laser processing, additional photo-generated carriers are formed. Their concentration will generally dominate the thermally created carriers. Free carriers may also be present as a result of doping the material.

\section{Lattice absorption}

For wavelengths longer than approximately $6 \mu \mathrm{m}$, laser energy is directly absorbed by the lattice [94]. As this prevents laser radiation from penetrating silicon, lattice absorption is undesirable for the formation of subsurface modifications.

\section{Tunnelling ionization}

In addition to linear and multiphoton absorption, tunnelling ionization [95] may contribute when tightly focussing short laser pulses inside bulk materials. It was found that even for near-infrared pulses as short as $100 \mathrm{fs}$, focussed inside silicon, the laser energy absorption 
shows a strong dependence on the wavelength [96]. This implies that multiphoton absorption is the dominant absorption process. Consequently, tunnelling ionization was neglected.

Energy storage after absorption Apart from lattice absorption, all absorption mechanisms discussed above are related to interactions between electrons and photons. As a result, the initial storage of absorbed energy will mainly be in the electronic subsystem in the form of [97]:

- Excess carrier energy, i.e. an increase in the kinetic energy of the free carriers.

- The generation of additional electron-hole pairs.

Finally, over time, the energy of the electrons is transferred to the lattice by the electronphonon coupling and photo-excited electron-hole pairs disappear due to recombination [97].

\subsection{Processing strategies}

An important aspect of the absorption mechanisms discussed in section 2.3 , for the formation of subsurface modifications, is to which extend they contribute towards the goal of triggering localised absorption of laser energy in the bulk of silicon. Based on the properties of these absorption mechanisms, two processing strategies may be employed to achieve this goal. In practise, depending on the wavelength of the laser source and the duration and energy of the laser pulses, a combination of both strategies may occur.

\section{Photon energy close to the band gap}

The first processing strategy is based on the selection of a photon energy that is close to the band gap of silicon, which requires a laser wavelength around $1.1 \mu \mathrm{m}$ (see section 2.3). The photon energy should be chosen such that a limited amount of linear absorption will occur. The absorptivity has to be low enough to allow the laser beam to propagate into a silicon sample. However, it should be high enough to cause moderate heating of the material. If the laser beam is focussed inside silicon, the temperature increase in the focus will be larger than in the surrounding material. Due to the temperature-dependence of the linear absorption coefficient, this means that the absorptivity in the focus will also be enhanced compared with the absorptivity elsewhere inside the material, resulting in an even faster increase in temperature. Consequently, a self-accelerating process known as a thermal runaway can be triggered [54].

Once a sufficiently high concentration of free carriers has been generated, free carrier absorption will contribute to the absorption of laser energy. Therefore, the selective generation of these carriers, in the volume that is to be modified, is of great importance for the subsurface modification process.

\section{Photon energy below the band gap}

The second processing strategy is to use a photon energy well below the band gap, corresponding to a wavelength above $1.1 \mu \mathrm{m}$. If the linear interband absorption is negligible at room temperature, multiphoton absorption is required to trigger the formation of a 
subsurface modification. Since multiphoton absorption depends on at least the square of the light intensity, silicon will behave transparent or opaque, depending on the local light intensity in the path of the laser beam. Due to this advantage, multiphoton absorption can be exploited for selective three-dimensional micromachining.

Since high laser intensities are required to induce multiphoton absorption, the Kerr effect also has to be considered (see section 2.3). It is important to ensure that instantaneous power levels beyond the critical power for self-focussing are not required to obtain efficient multiphoton absorption in the vicinity of the focus of the beam.

\subsection{Time scales of physical phenomena}

During the laser-material interaction inside silicon, many physical processes take place related to the energy exchange between photons, electrons and phonons. When developing a numerical model to investigate the laser-material interaction, the relevant physical phenomena should be included. Moreover, it is beneficial to neglect physical phenomena that are too slow or too fast to be of importance, to limit the complexity of the model and to reduce the computational load. When comparing the duration of the laser pulse with the time scale of physical processes, the following approximations can be made when modelling the behaviour of a laser pulse in a solid:

- Phenomena that have a significantly faster characteristic time scale than the duration of the laser pulse can be assumed to occur instantaneously.

- Processes that occur on a significantly longer time scale than the duration of the laser pulse can be neglected altogether.

If not only the dynamics during the laser pulse but also the physical phenomena that occur after the laser pulse are simulated, the second assumption should not be applied.

An overview of the characteristic time scales in the photon-electron-phonon coupling in semiconductors is shown in figure 2.3. As can be observed, the carrier-phonon scattering, i.e. the transfer of energy from excited electrons to the lattice, occurs on a lower pico- to femtosecond time scale. One of the objectives of this thesis is to investigate the suitability of ultra-short pico- to femtosecond pulses for the formation of subsurface modifications in silicon. Therefore, only the absorption of photons and the carrier-carrier scattering were assumed to occur instantaneously in the numerical model (see section 3.2). Since pulse durations beyond $100 \mathrm{~ns}$ have been applied for the formation of subsurface modifications in silicon (see section 1.5), none of the relatively slow phenomena in figure 2.3 were neglected.

\subsection{Modification mechanisms}

In this section, potential mechanisms to change the crystal structure of bulk crystalline silicon are discussed. Since only silicon atoms are involved in the process, chemical reactions between different elements can be excluded. Consequently, the possible types of modifications are: 


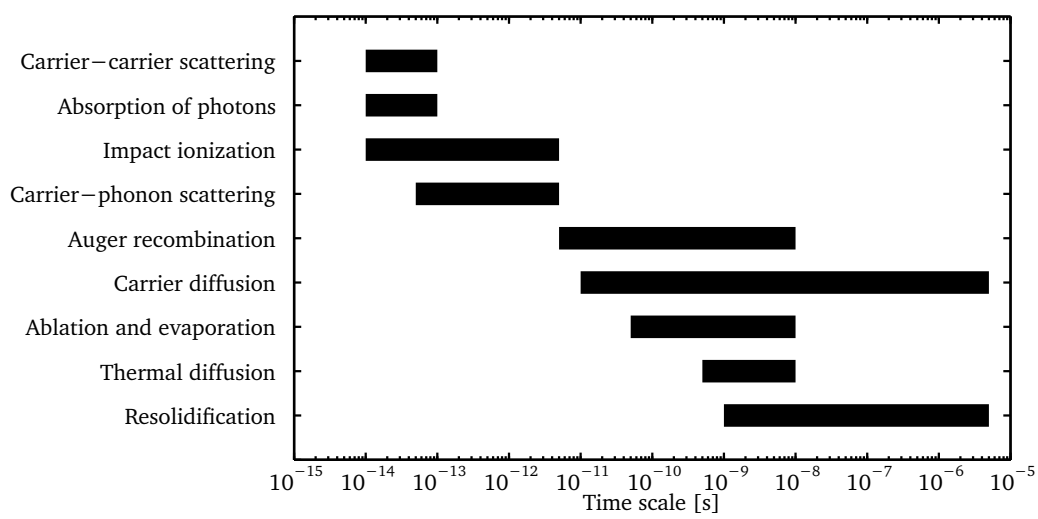

Figure 2.3: Approximate time scales of physical phenomena related to the processing of semiconductors by pulsed lasers. The data were obtained from reference [97]. The bars indicate the time ranges corresponding to concentrations of free carriers between $10^{17}$ and $10^{22} \mathrm{~cm}^{-3}$.

- A transformation from the monocrystalline diamond cubic phase to other crystalline, polycrystalline or amorphous phases. A transition to an amorphous and/or polycrystalline phase is often encountered after laser irradiation of crystalline silicon [65, 73, $98-100]$.

- A change in the density of microscopic lattice defects such as dislocations or stacking faults.

- The formation of macroscopic geometrical faults such as voids or cracks.

The changes that are listed above can be permanent, meaning that they remain after the laser pulse and are (meta)stable at room temperature and atmospheric pressure. Additionally, they may also disappear during or shortly after the laser pulse. An overview of the driving forces that may be responsible for changes to the material structure is given below.

\section{Thermal melting and resolidification}

Diamond cubic silicon will melt if the temperature exceeds $1687 \mathrm{~K}$ [101]. Melting is often found to be an accurate indicator for laser damage of silicon, as the fast resolidification that occurs after the pulse prevents the silicon from returning to its original low-defect monocrystalline state $[72+-75]$. The resolidification speed determines whether silicon can recrystallize into its original state or whether a permanent modification is left behind [102].

\section{Mechanical failure}

Mechanical failure of silicon, in the absence of defects, occurs when the tensile stress exceeds the ultimate tensile strength of $7 \mathrm{GPa}$ [103]. While silicon is brittle at room temperature, plastic deformation of crystalline silicon may also occur above the brittle to ductile transition, which takes place at a temperature around $1000 \mathrm{~K}$ [104]. The exact transition temperature depends on the dopant concentration [104]. 


\section{Pressure-induced phase transitions}

Apart from causing mechanical failure, stresses may also induce phase transitions. If the hydrostatic stress in silicon exceeds $8.8 \mathrm{GPa}$, a pressure-induced phase transition will occur, with several further transitions taking place at even higher pressures (see section 2.2).

The generation of pressures in the TPa range has been linked to confined microexplosions in the vicinity of the focus of the laser beam [8,-10, 24]. That is, a shock wave may be formed by the vaporization of a small volume of material below the surface [8]. This pressure wave could cause pressure-induced phase transformations in the vicinity of the vapour bubble. When the density of the material surrounding the vaporized volume is increased, a void is left behind [8].

\section{Non-thermal melting}

When a significant percentage of the electrons in the valence band are transferred to the conduction band, the silicon lattice becomes unstable, resulting in a non-thermal transition to the liquid phase. This is expected to occur when approximately $9 \%$ of the electrons have been removed from the valence band [105].

\subsection{Wafer separation mechanisms}

The modification mechanisms discussed in section 2.6 are related to the phenomena that occur during the laser-material interaction. However, the second step of the subsurface dicing process, when the dies are separated by exerting an external force on the wafer, is also of interest. At this stage, the mechanical failure of the wafer along predefined tracks is the desired outcome, i.e. the silicon should fracture along the dicing streets. Several mechanisms may contribute to the breaking of the wafer along the laser-modified tracks. An overview of these mechanisms is given below.

- A superposition of residual stresses and stresses as a result of external forces that together exceed the ultimate strength of silicon. Residual stresses may be present due to differences in density between the unmodified material and material phases that were created during the laser-material interaction. High pressure phases are denser than diamond cubic silicon [106], while both low and high density amorphous phases exist, depending on the pressure at which they were formed [79].

- Failure due to crack growth, induced by stress concentrations at the tips of microcracks that were created during the laser-material interaction.

- Failure due to solid phases that have a lower strength compared with the surrounding material that is still in the original crystalline phase.

- Failure due to cleavage along boundaries between different material phases.

The orientation of the subsurface modifications with respect to the crystallographic planes of the wafer is likely to play a role during separation. When introducing a line defect or a point defect on the surface of a silicon wafer, this defect will usually initiate cleavage along one of the lattice planes, when a force is applied on the wafer. Cleavage will generally 
occur along the $\{111\}$ planes [78] (see figure 2.1). For the subsurface dicing process to succeed, the wafer should fail along the planes containing the laser-induced modifications and inadvertent cleavage along $\{111\}$ planes has to be prevented.

\subsection{Processing conditions}

Several process parameters can be adapted to enable or optimize the formation of subsurface modifications in silicon. The most important laser parameters are the wavelength $\lambda_{0}$, the pulse duration $t_{p}$ and the pulse energy $E_{p}$. The pulse energy and pulse duration will be selected based on simulation results (see chapter 4).

The selection of the wavelength depends on the processing strategy that is employed. For the process that requires a limited amount of single-photon interband absorption (see section 2.4), a wavelength around $1.1 \mu \mathrm{m}$ is needed, which corresponds to a photon energy that is close to the band gap of silicon. The relevant wavelengths for the multiphoton process range from approximately $1.2 \mu \mathrm{m}$ to $6 \mu \mathrm{m}$, to prevent linear interband absorption at short wavelengths and lattice absorption at long wavelengths (see section 2.3).

For the choice of the wavelength, the availability of laser sources also has to be taken into account. For research applications, the wavelength can be adapted by means of optical parametric amplification [107]. However, this technology has a low efficiency and comes at a significant cost. Therefore, the application of laser sources that directly provide the required photon energy was preferred, to allow the laser process to be integrated in industrial equipment. When considering well-known industrial laser sources, the Yb:YAG, $\mathrm{Nd}$ :Glass, Nd:YAG, Nd:YVO $\mathrm{YN}_{4}$ and "Yb:Silica fibre" lasers provide wavelengths between 1030 and $1080 \mathrm{~nm}$ [85], corresponding to a photon energy just above the band gap of silicon. Within the wavelength range for the multiphoton process, the "Er:Silica fibre" laser, operating at approximately $1550 \mathrm{~nm}$ [85], is the most common source. At this wavelength, the absorption in silica fibres is minimal [108]. Consequently, it is frequently used in long distance telecommunications. The wavelength window around $1550 \mathrm{~nm}$ is referred to as the conventional or C-band in optical communications [108]. Because of its use in telecommunications, there is a good supply of laser sources, cameras and optical components for the $1550 \mathrm{~nm}$ wavelength.

Based on the availability of laser sources and their potential suitability for the formation of subsurface modifications in silicon, two wavelengths of 1064 and $1550 \mathrm{~nm}$ were selected to be evaluated by numerical simulations (see chapter 4) and experiments (see chapter 6). These can be generated by Nd:YAG or "Yb:Silica fibre" and "Er:Silica fibre" lasers respectively. Detailed information about the specific lasers sources that were employed for the experiments can be found in section 5.2 .

\subsection{Summary}

An overview has been given of the physics that govern the laser-material interaction, when laser pulses are focussed inside crystalline silicon. The main requirement for the production of subsurface modifications inside a silicon wafer is that the laser energy can be selectively 
absorbed in a small volume in the bulk of the material. Based on the mechanisms of laser energy absorption, two strategies to achieve this goal were identified.

The first strategy consists of selecting a photon energy near the band gap of silicon. This results in a limited amount of single-photon interband absorption. The absorption should be low enough to allow the laser beam to reach the focus inside silicon, without too much absorption in the preceding beam path. However, the absorption at room temperature should be high enough to induce an initial temperature increase near the location of the focus of the laser beam. As the single-photon interband absorption coefficient strongly increases with temperature, a thermal runaway can be triggered.

The second strategy is based on a photon energy well below the band gap. For such photon energies, linear interband absorption at room temperature is negligible. However, interband absorption is still possible by means of multiphoton absorption. Since multiphoton absorption depends on the square or higher powers of the light intensity, this mechanism allows for the laser energy to be selectively absorbed.

Two wavelengths of 1064 and $1550 \mathrm{~nm}$ were selected, based on their potential suitability for the single- and multiphoton processes respectively and the fact that they match the wavelengths of common industrial laser sources. In chapters 4 and 6 , the suitability of these wavelengths for the formation of subsurface modifications in silicon will be evaluated by numerical simulations and experiments. 


\section{Numerical model for laser-material interaction in bulk silicon}

In this chapter,* a numerical model to simulate the laser-material interaction during the formation of subsurface modifications in silicon is proposed. The governing equations that describe the response of the material to the absorption of laser energy will be considered. Moreover, the equations that were used to simulate the laser beam propagation are discussed.

\subsection{Introduction}

The primary goal of the model that is proposed in this chapter is to select suitable processing conditions for the formation of subsurface modifications in crystalline silicon (see section 1.6). While models to simulate certain aspects of the laser-material interaction inside silicon exist (see section 1.5), none of these are capable of quantitatively predicting whether subsurface modifications will be formed using a specific set of processing conditions. A secondary goal is to obtain a reasonable match between numerically computed and experimentally obtained shapes of the modifications, as it is beneficial to not only predict whether a modification will be formed but also to estimate its geometry.

The physics relevant for the processing with wavelengths between one and two micrometres were modelled, as this range includes the laser sources that were considered to be of practical use (see section 2.8). Moreover, it includes wavelengths that are suitable for both processing strategies that were discussed in section 2.4 .

In the next section, the equations that describe the distributions of the lattice and electron temperatures and the density of free carriers will be discussed. After that, the model to simulate the propagation of the laser beam is introduced. Finally, the numerical solvers and material properties are considered.

\subsection{Two-temperature model}

Based on the time scales of the relevant physical phenomena and the laser pulse durations that the model should be able to simulate (see section 2.5), a two-temperature model (тTM) [109] was selected. A тTM accounts for the fact that the energy of photo-excited electrons is not instantaneously converted into lattice heat, but remains in the electronic subsystem for a certain period of time. This implies that the time delay in the carrier-phonon scattering is modelled, enabling detailed simulations of the laser-material interaction during ultra-short pico- to femtosecond pulses. Only the absorption of photons and the carriercarrier scattering were assumed to occur instantaneously.

\footnotetext{
*Parts of the numerical model have been published in Applied Physics A: Materials Science \& Processing, 144(4):1135-1143, 2014 (P. C. Verburg, G. R. B. E. Römer, and A. J. Huis in 't Veld).
} 
A ттм includes separate distributions for the lattice and electron temperatures in the material. Apart from the temperatures, the carrier density was modelled. This is required because additional free carriers are generated during the laser pulse, due to electrons transferring from the valence to the conduction band upon the absorption of photons or by impact ionization.

Based on the above, a conservation equation has to be solved for each of the following distributions in the material: (1) the density of free carriers, (2) the lattice temperature and (3) the electron temperature. In this section, these equations will be derived. Additionally, the lattice temperature was coupled to the mechanical domain, in order to compute the stresses and strains as a result of thermal expansion. Finally, the modification mechanisms that were included in the numerical model will be discussed.

\subsubsection{Density of free carriers}

The material is considered to remain locally charge neutral during processing, i.e. the concentrations of electrons and holes are identical. This is a reasonable assumption, as charge separation generates a counteracting electric field, preventing significant differences between the electron and hole currents [72]. This leads to the following conservation law for the electron-hole pairs:

$$
\frac{\partial N}{\partial t}+\nabla \cdot \vec{q}_{N}=Q_{N}
$$

in which $N$ denotes the density of electron-hole pairs, $t$ the time, $\vec{q}_{N}$ the current and $Q_{N}$ the source term. The latter includes the generation and recombination of electron-hole pairs. The current $\vec{q}_{N}$ follows from [72]

$$
\vec{q}_{N}=-k_{N} \nabla N
$$

where $k_{N}$ is the ambipolar diffusivity of the material. Here, the Seebeck effect and the effect of spatial variations in the band gap were neglected. The ambipolar diffusivity, based on the Einstein relation, is given by [110]

$$
k_{N}=\frac{k_{e} \mu_{h}+k_{h} \mu_{e}}{\mu_{e}+\mu_{h}}=\frac{2 k_{B} T_{l}}{e} \frac{\mu_{e} \mu_{h}}{\mu_{e}+\mu_{h}},
$$

where $k$ is the diffusivity, $\mu$ the mobility, $k_{B}$ the Boltzmann constant, $T_{l}$ the lattice temperature, and $e$ the elementary charge. The subscripts $e$ and $h$ refer to the electrons and holes respectively.

The mechanism of non-thermal melting [97,111] has been taken into account by assuming the material to be molten if $N$ exceeds the critical value $N_{\text {crit }}$, the latter being a material property.

\subsubsection{Lattice temperature}

The conservation of lattice heat is given by the heat equation:

$$
\rho c_{\text {app }} \frac{\partial T_{l}}{\partial t}+\nabla \cdot \vec{q}_{l}=\gamma\left(T_{e}-T_{l}\right),
$$


in which $T_{l}$ denotes the lattice temperature, $\rho$ the density, $c_{\text {app }}$ the apparent lattice specific heat capacity, $\vec{q}_{l}$ the lattice heat flux and $\gamma$ the electron-phonon coupling coefficient. The only source of lattice heat originates from the electron-phonon coupling. The flux $\vec{q}_{l}$ is described by Fourier's law:

$$
\vec{q}_{l}=-k_{l} \nabla T_{l},
$$

with $k_{l}$ the thermal conductivity of the lattice.

The latent heat of fusion is accounted for in the apparent heat capacity [112] as

$$
c_{\mathrm{app}}= \begin{cases}\frac{\int_{T_{s}}^{T_{f}} c_{p} d T_{l}+L_{m}}{2 \Delta T} & T_{s}<T_{l}<T_{f} \\ c_{p} & \text { otherwise }\end{cases}
$$

where $c_{p}$ is the specific heat capacity and $L_{m}$ the latent heat of fusion. Further, $\Delta T$ represents a mushy temperature interval, to model the solid-liquid transition. The solid and fluid temperatures are defined as $T_{s}=T_{m}-\Delta T$ and $T_{f}=T_{m}+\Delta T$ respectively, with $T_{m}$ the melting temperature. A similar procedure was applied when simulating the liquid-vapour transition. Undercooling of the melt was not taken into account, as the detailed simulation of the solidification of molten material is beyond the scope of the model.

\subsubsection{Electron temperature}

To derive the conservation equation for the electron temperature, first a conservation equation for the total energy density $U$ of the electron-hole pairs has to be defined. This energy density is the sum of the kinetic and band gap energy of the electron-hole pairs [72], which reads

$$
U=3 k_{B} T_{e} N+E_{g} N,
$$

where $T_{e}$ is the electron temperature and $E_{g}$ is the band gap energy. The kinetic energy of the electrons was assumed to show a Boltzmann distribution. This choice was made because, for the ultra-short pulses for which a two-temperature model is relevant, high carrier densities are accompanied by high electron temperatures. Therefore, the electron-hole plasma will not be highly degenerate [72]. The specific heat capacity of the electron-hole pairs $C_{e}$ then reads

$$
C_{e}=\left.\frac{\partial U}{\partial T_{e}}\right|_{N}=3 k_{B} N
$$

The conservation equation for the electronic energy is

$$
\frac{\partial U}{\partial t}+\nabla \cdot \vec{W}=Q_{e}-\gamma\left(T_{e}-T_{l}\right)
$$

with $\vec{W}$ the electronic heat flux and $Q_{e}$ the source term representing all absorbed laser energy. To obtain a conservation equation for the electron temperature, equations (3.1), (3.7) and (3.8) are substituted into equation (3.9), which yields

$$
C_{e} \frac{\partial T_{e}}{\partial t}+\nabla \cdot \vec{W}=Q_{e}-\gamma\left(T_{e}-T_{l}\right)-\left(E_{g}+3 k_{B} T_{e}\right) \frac{\partial N}{\partial t}-N \frac{\partial E_{g}}{\partial t} .
$$


The electronic heat flux $\vec{W}$ consists of two terms [72]. The first term is the electron-hole current $\vec{q}_{n}$ multiplied by the sum of the band gap and the Peltier coefficient. The second term is the electronic thermal conduction $\vec{q}_{e}$, such that

$$
\vec{W}=\vec{q}_{n}\left(E_{g}+4 k_{B} T_{e}\right)+\vec{q}_{e} .
$$

Like the lattice heat flux (3.5), the electronic thermal conduction $\vec{q}_{e}$ is described by Fourier's law:

$$
\vec{q}_{e}=-k_{e} \nabla T_{e}
$$

where $k_{e}$ is the electronic thermal conductivity, which follows from the Wiedemann-Franz law [113]:

$$
k_{e}=\frac{2 k_{B}^{2} T_{e} N\left(\mu_{e}+\mu_{h}\right)}{e} .
$$

\subsubsection{Source terms}

In this section, the source terms $Q_{N}, \gamma$ and $Q_{e}$ in equations (3.1), 3.4 and (3.10) respectively will be derived. For the electron-phonon coupling a relaxation-time model was used [72], which implies that

$$
\gamma=\frac{C_{e}}{\tau_{e}}
$$

Here $\tau_{e}$ is the electron-phonon relaxation time.

Next, the carrier density source term $Q_{N}$ in equation (3.1) is to be determined. This term includes all mechanisms that generate or remove carriers. First, interband absorption will be described, based on ionization due to linear and multiphoton absorption of laser energy. Tunnelling ionization was neglected (see section 2.3). Each single or two-photon absorption event creates a free carrier, which yields the source term

$$
Q_{N_{\mathrm{phot}}}=\frac{\alpha I}{E_{\mathrm{phot}}}+\frac{\beta I^{2}}{2 E_{\mathrm{phot}}},
$$

with $\alpha$ the linear interband absorption coefficient, $\beta$ the two-photon absorption coefficient and $I$ the laser intensity. It is noted that $\alpha$ is strongly temperature-dependent (see section 2.3..$E_{\text {phot }}$ is the photon energy, which follows from the Planck relation:

$$
E_{\text {phot }}=\frac{h c_{0}}{\lambda_{0}}
$$

where $h$ is Planck's constant, $c_{0}$ the speed of light in vacuum and $\lambda_{0}$ the wavelength of the laser light in vacuum.

At the high carrier densities that are encountered during laser-material interaction, Auger recombination is dominant in silicon [114]. Therefore, radiative recombination [115], Shockley-Read-Hall recombination [116] and surface recombination [117] were neglected. During Auger recombination, the recombination energy is transferred to a free carrier. As 
an electron, a hole and another free carrier are involved, the source term of this process is expressed by a third order function [114]:

$$
Q_{N_{\text {Auger }}}=\gamma_{3} N^{3},
$$

in which $\gamma_{3}$ denotes the Auger recombination coefficient.

Additionally, impact ionization can occur at high electron temperatures and carrier densities. It is described by [72]

$$
Q_{N_{\text {impact }}}=\delta N,
$$

where $\delta$ is the impact ionization coefficient. The complete source term of the free carrier distribution is then given by

$$
Q_{N}=Q_{N_{\text {phot }}}-Q_{N_{\text {Auger }}}+Q_{N_{\text {impact }}} .
$$

Finally, a source term $Q_{e}$ is required for equation (3.10), describing the conservation law for the electron temperature. This term includes all energy absorbed from the laser beam:

$$
Q_{e}=\alpha I+\beta I^{2}+\sigma N I,
$$

in which $\sigma$ is the free carrier cross-section. Lattice absorption was neglected, as it does not provide a significant contribution within the wavelength range under consideration (see section 2.3).

\subsubsection{Mechanical simulations}

The two-temperature model was coupled to a mechanical model, in order to simulate thermally-induced stresses and strains in the solid phase. The mechanical phenomena were assumed to be quasi-static, i.e. the effect of inertia was neglected.

The relation between the stresses and strains is expressed as

$$
\vec{\sigma}=\mathbf{E} \vec{\epsilon}
$$

where $\vec{\sigma}$ is an array containing the stresses, $\vec{\epsilon}$ is an array containing the strains and $\mathbf{E}$ is the constitutive matrix. For the simulations, an axisymmetric coordinate system was applied and the material was assumed to be isotropic (see section 3.4.1). The coordinate along the optical axis is $z$, while $r$ and $\theta$ are the radial and azimuthal coordinates respectively. In this specific case, equation (3.21) can be written as [118

$$
\left\{\begin{array}{c}
\sigma_{r} \\
\sigma_{\theta} \\
\sigma_{z} \\
\tau_{z r}
\end{array}\right\}=\frac{(1-v) E}{(1+v)(1-2 v)}\left[\begin{array}{cccc}
1 & f & f & 0 \\
f & 1 & f & 0 \\
f & f & 1 & 0 \\
0 & 0 & 0 & g
\end{array}\right]\left\{\left\{\begin{array}{c}
\epsilon_{r} \\
\epsilon_{\theta} \\
\epsilon_{z} \\
\gamma_{z r}
\end{array}\right\}-\left\{\begin{array}{c}
\epsilon_{t} \\
\epsilon_{t} \\
\epsilon_{t} \\
0
\end{array}\right\}\right),
$$

where $E$ is Young's modulus, $v$ is Poisson's ratio and $\epsilon_{t}$ is the strain due to thermal expansion. Moreover, the normal stresses and strains are described by $\sigma$ and $\epsilon$ respectively, while $\tau$ and $\gamma$ are the shear stresses and strains. Further, $f$ and $g$ are defined as [118]

$$
f=\frac{v}{1-v} \quad \text { and } \quad g=\frac{1-2 v}{2(1-v)}
$$


For a constant linear thermal expansion coefficient $\alpha_{L}$, the thermal strain is given by

$$
\epsilon_{t}=\alpha_{L} \Delta T
$$

where $\Delta T$ is the change in temperature. Since the thermal expansion coefficient of silicon is temperature-dependent (see section 3.1), equation (3.22) was used to evaluate the incremental stresses for a certain time step. In the incremental formulation, equation (3.24) is employed to compute the incremental thermal strains, based on the change in temperature during these steps. This temperature change should be sufficiently small, such that the values of $\alpha_{L}$ corresponding to the temperatures at the beginning and end of the step are close to each other.

\subsubsection{Modification mechanisms}

Multiple modification mechanisms may play a role during the formation of a subsurface modification. For the numerical simulations, the aim was to include the primary modification mechanisms that occur first during the laser pulse, as they determine the feasibility of creating subsurface modifications. Additional secondary mechanisms may, however, have an effect on the final geometry and material structure of the subsurface modifications. In other words, primary mechanisms are a necessary condition to initiate the formation of modifications, while secondary mechanisms can contribute to their growth.

In the discussion on modification mechanisms in section 2.6 the lattice temperature, stresses and the electronic configuration were identified as the driving forces behind the subsurface modification of silicon. All three phenomena have been included in the numerical simulations.

It is known from literature that surface melting is an accurate indicator for surface modification of monocrystalline silicon wafers $[72-76]$. This is a result of the rapid resolidification that occurs, which prevents the silicon from regaining its nearly defect-free monocrystalline structure [102]. Similar high cooling rates occur when a subsurface liquid volume is surrounded by cold material. Therefore, it is reasonable to assume that once a subsurface liquid volume has been formed, a modification will be created. Based on this hypothesis, the numerical model was designed to accurately predict the temperatures, carrier density, beam propagation and stresses and strains inside silicon in the solid phase, as this choice allows all potential primary modification mechanisms to be included. Since melting was considered to induce a modification, any subsequent mechanism that occurs after melting is by definition a secondary mechanism. A potential secondary modification mechanism, which is not included in the model, is a confined microexplosion (see section 2.6).

\subsection{Beam propagation model}

To evaluate the source terms that were described in section 3.2.4 the distribution of the laser intensity inside the material is required. Therefore, the propagation of the laser beam has to be simulated. In this section, the equations that were employed for this purpose will be discussed. First, a description of the temporal profile of the laser beam is provided, 
followed by an overview of the approximations that were applied in the beam propagation model. Next, the propagation equations will be derived starting from Maxwell's equations. After that, a mathematical description of the beam emitted by the laser source will be presented. Last, an overview is provided of how the different parts of the laser beam path, starting from the laser source to the bulk of the sample, are modelled.

\subsubsection{Laser power}

Two different temporal pulse shapes of the laser beam were implemented in the numerical model. The shape that provides the best match to the temporal shape of the actual laser pulse will be employed, when comparing numerical results with experimental data.

The first pulse shape assumes a constant power during the pulse. For this shape, the power $P$ is defined by

$$
P=\frac{E_{p}}{t_{p}} \quad \text { for } t=0 \ldots t_{p},
$$

where $E_{p}$ is the on-sample pulse energy and $t_{p}$ is the pulse duration. Since $E_{p}$ represents the energy that reaches the sample that is being processed, the power $P$ is defined as the power after the focussing objective. The actual power emitted by the laser source may be higher if significant aperture losses are present.

The second pulse shape is a Gaussian profile. In this case, the laser power as a function of time is given by

$$
P=\frac{E_{p}}{\sigma \sqrt{2 \pi}} \exp \left[-\left(\frac{t-1 / 2 t_{\text {sim }}}{\sqrt{2} \sigma}\right)^{2}\right],
$$

where $t_{\text {sim }}$ is the time period that is simulated and $t$ is in the range of $0 \ldots t_{\text {sim }}$. The peak power occurs at $t=1 / 2 t_{\text {sim. }}$. $\sigma$ is similar to the standard deviation of a normal distribution and is defined as

$$
\sigma=\frac{t_{p}}{2 \sqrt{2 \ln (2)}} .
$$

Here, $t_{p}$ is the full duration at half maximum (FDHM) pulse duration. In order to ensure that the laser power at the beginning and end of the simulation was negligible compared with the peak power, $t_{\text {sim }}$ was set to $4 t_{p}$.

As the propagation equations that were applied are unidirectional, they do not take reflection losses into account (see section 3.3.5). Therefore, the power of the beam was reduced by a factor $1-R$, where $R$ is the reflectivity of the material.

\subsubsection{Assumptions}

To obtain a beam propagation model that can be implemented efficiently in numerical simulations on a nanosecond time scale, a number of assumptions have been applied:

1. The medium is non-dispersive and isotropic.

2. Solutions of the wave equation for the components of the electric and magnetic fields satisfy Maxwell's equations for a linear, non-dispersive, isotropic and homogeneous medium. 
3. The light wave is monochromatic.

4. The material properties vary gradually in space compared with the wavelength of the light.

5. The effect of the polarization may be neglected.

6. The optical rays in the laser beam propagate at small angles with respect to the optical axis.

7. The variations in the real part of the refractive index, excluding interfaces, are limited.

The necessity of these assumptions, even though they introduce deviations from the physical reality, lies in the vast reduction of the computational effort that can be achieved. They allow the propagation of the laser beam to be modelled by a nonlinear Schrödinger equation (see section 3.3.6).

For the subsurface processing of silicon, pulsed lasers will be employed. As given by the Fourier transform, a light wave can only be monochromatic if it is stationary. Because of the use of pulsed lasers and the fact that the material properties change over time, the amplitude of the light wave is modulated in time. Consequently, the monochromatic assumption induces some errors. For pico- to nanosecond pulses, the modulation of the wave amplitude is slow compared with the oscillations of the optical wave. Even when considering the propagation of pulses with a duration of 250 femtoseconds in silicon, it was found that neglecting the temporal gradients did not significantly affect the outcome of the simulations [71]. Therefore, the assumption of monochromaticity is considered to be reasonable.

The only fully reliable method to assess the impact of the assumptions in the beam propagation model would be to perform full Maxwell simulations. Within the current capabilities of computer systems, a full solution of Maxwell's equations (see section 3.3.3. on a nanosecond time scale is a formidable task. While this was considered to be infeasible for complete laser pulses, a solution for the simplified case of a homogeneous non-absorbing medium, which satisfies Maxwell's equations, will be presented in section 4.2. This solution enables a partial verification of the propagation model.

\subsubsection{Maxwell's equations}

On a macroscopic scale, the most general description of the propagation of an electromagnetic wave inside a material, in the field of classical electrodynamics, is provided by Maxwell's equations. Maxwell's equations in a source-free medium are given by [85]

$$
\begin{aligned}
\nabla \times \vec{H} & =\frac{\partial \vec{D}}{\partial t}, \\
\nabla \times \vec{E} & =-\frac{\partial \vec{B}}{\partial t}, \\
\nabla \cdot \vec{D} & =0, \\
\nabla \cdot \vec{B} & =0,
\end{aligned}
$$


where $\vec{H}$ is the magnetic field, $\vec{E}$ the electric field, $\vec{B}$ the magnetic flux density and $\vec{D}$ the electric flux density. The fields and flux densities are related by [85]

$$
\begin{aligned}
& \vec{D}=\epsilon_{0} \vec{E}+\vec{P}, \\
& \vec{B}=\mu_{0} \vec{H}+\mu_{0} \vec{M},
\end{aligned}
$$

where $\vec{P}$ and $\vec{M}$ are the polarization and magnetisation density respectively, which have to be expressed as functions of the fields by means of constitutive relations that describe the properties of the material. In vacuum, both $\vec{P}$ and $\vec{M}$ are equal to zero. Further, $\epsilon_{0}$ is the vacuum permittivity and $\mu_{0}$ is the vacuum permeability, which are both physical constants.

When the medium that is under consideration is dielectric, linear, isotropic, homogeneous and non-dispersive, the polarization density $\vec{P}$ is related to the electric field by [85]

$$
\vec{P}=\epsilon_{0} \chi \vec{E}
$$

in which $\chi$ is the electric susceptibility of the medium. The medium being isotropic and non-dispersive was listed as assumption (1) in section 3.3.2. Conduction effects and inhomogeneous material properties will be dealt with at the bottom of this section. Nonlinear effects are considered in section 3.3.6. Substitution of equation (3.34 in equation 3.32) yields

$$
\vec{D}=\epsilon \vec{E}
$$

where $\epsilon$ is the permittivity of the medium which is defined by [85]

$$
\epsilon=\epsilon_{0}(1+\chi)
$$

Similarly, for the magnetic flux density:

$$
\vec{B}=\mu \vec{H},
$$

where $\mu$ is the permeability of the medium. Both the permittivity and permeability can be complex due to the effect of absorption.

In a Cartesian coordinate system, substitution of equations (3.35) and (3.37) in Maxwell's equations (3.28)-3.31), yields that a necessary condition for each component of $\vec{E}$ and $\vec{H}$ to satisfy Maxwell's equations is that they satisfy the wave equation, which reads

$$
\nabla^{2} u-\frac{1}{c^{2}} \frac{\partial^{2} u}{\partial t^{2}}=0
$$

Here, $u$ represents any of the field components and $c$ is the speed of light in the medium, which follows from

$$
c=\frac{1}{\sqrt{\epsilon \mu}} .
$$

The derivation of the wave equation (3.38) from Maxwell's equations constitutes the connection between electromagnetic and wave optics. However, the requirement that the field components satisfy the wave equation is a necessary but not a sufficient condition [119]. Therefore, the validity of continuing the derivation of the beam propagation model based on the wave equation instead of Maxwell's equations was listed as assumption (2). 
Effect of conductivity Equation (3.34) is based on the assumption that the medium is dielectric. However, conductivity effects are also relevant in silicon, especially when a high concentration of photo-ionized carriers is present. According to assumption (3), the wave is monochromatic. For a monochromatic wave, Maxwell's equations for a dielectric medium are still applicable for a conducting medium, when replacing the permittivity $\epsilon$ by an effective value $\epsilon_{\text {eff }}[85]$. This value is defined as

$$
\epsilon_{\mathrm{eff}}=\epsilon+\frac{\sigma}{j \omega}
$$

in which $\omega$ is the angular frequency of the monochromatic wave, $j$ is the imaginary unit and $\sigma$ is the electrical conductivity.

Inhomogeneous material properties The wave equation 3.38 was derived for homogeneous media. However, in the case of the interaction of a laser beam with a semiconductor, the material properties will be inhomogeneous due to laser-induced gradients in the temperature and carrier density. This implies that in equation (3.35), the electric permittivity is position-dependent. In a Cartesian coordinate system, substitution of a position-dependent permittivity in Maxwell's equations yields [85]

$$
\nabla^{2} \vec{E}-\frac{1}{c^{2}} \frac{\partial^{2} \vec{E}}{\partial t^{2}}+\nabla\left(\frac{1}{\epsilon} \nabla \epsilon \cdot \vec{E}\right)=0 .
$$

The requirement that each field component should satisfy the wave equation 3.38 follows from the first two terms on the left hand side of equation (3.41). According to assumption (4), $\epsilon$ varies gradually with respect to the wavelength of the light. Therefore, the third term in equation (3.41) is negligible [85], implying that the wave equation remains approximately valid.

\subsubsection{Helmholtz equation}

Since the wave is monochromatic, as stated in assumption (3) in section 3.3.2, each component of the electric and magnetic field will oscillate at the same frequency, allowing the solution of equation (3.38) to be split into space and time contributions:

$$
u(\vec{x}, t)=\Re[g(t) \tilde{U}(\vec{x})],
$$

with $\vec{x}$ being a vector containing the spatial coordinates. Here, $\tilde{U}$ is referred to as the complex amplitude, which is complex as it describes both the phase and amplitude of the wave. Further, $g(t)$ is defined as

$$
g(t)=e^{-j \omega t}
$$

Substitution of equations (3.39), 3.42 and (3.43) in equation 3.38) yields the Helmholtz equation:

$$
\nabla^{2} \tilde{U}+k^{2} \tilde{U}=0
$$

in which $k$ is the wavenumber, which is defined as

$$
k=\omega \sqrt{\epsilon \mu} .
$$


The wavenumber is complex when absorption is present. It is related to the complex refractive index $\tilde{n}$ by [85]

$$
k=\frac{\tilde{n} \omega}{c_{0}}=\frac{2 \pi \tilde{n}}{\lambda_{0}},
$$

where $c_{0}$ is the speed of light in vacuum. The real and imaginary parts of $\tilde{n}$ are given by [85]

$$
\Re(\tilde{n})=n \quad \text { and } \quad \mathfrak{I}(\tilde{n})=-\frac{c_{0} \alpha_{\text {tot }}}{2 \omega},
$$

where $n$ is the real refractive index and $\alpha_{\text {tot }}$ is the total absorption coefficient. The different physical phenomena that affect the refractive index and absorptivity can be found in section 2.3 .

Since in addition to the wave being monochromatic, the polarization is not considered according to assumption (5), the intensity of the wave can be derived from a single complex scalar equation [120], instead of solving the Helmholtz equation for each component of the field. The intensity $I$ can be directly extracted from the complex amplitude $\tilde{U}$ by [85]

$$
I=|\tilde{U}|^{2}
$$

\subsubsection{Paraxial Helmholtz equation}

The solution $\tilde{U}$ of the Helmholtz equation (3.44) still contains harmonic oscillations in space related to the wavelength of the light, as the phase of the wave oscillates along its propagation direction. Such oscillations are undesirable as they are difficult to accurately represent on a discrete computational grid. Additionally, the Helmholtz equation supports wave propagation in any direction, implying that the solution at a specific location depends on the solution anywhere else in the space domain. This is a complicating factor when developing a numerical solver.

Based on assumption (6) that the rays make small angles with the optical axis (see section 3.3.2), the solutions of the paraxial Helmholtz equation provide good approximations of the solutions of the Helmholtz equation. Moreover, the paraxial Helmholtz equation is not affected by the issues discussed above. Therefore, in this section, the paraxial form of the Helmholtz equation will be derived.

First, without introduction any approximation, a plane carrier wave propagating in $z$-direction is factored out of the scalar complex amplitude $\tilde{U}$ :

$$
\tilde{U}=e^{-j k_{0} z} \tilde{u},
$$

in which $\tilde{u}$ is the complex wave envelope, $k_{0}$ is a reference wavenumber and $z$ is the coordinate along the primary propagation direction of the wave. Since the carrier wave is of unit intensity, the intensity of the wave $\tilde{u}$ can be computed similar to equation (3.48):

$$
I=|\tilde{u}|^{2} .
$$

In equation 3.49, $k_{0}$ is the reference wavenumber, which is defined by

$$
k_{0}=\frac{n_{0} \omega}{c_{0}}=\frac{2 \pi n_{0}}{\lambda_{0}},
$$


where $n_{0}$ is the reference refractive index. At this stage, the choice of the reference refractive index is arbitrary. It only affects the smoothness of the solution $\tilde{u}$. If $\tilde{U}$ represents a wave travelling primarily in $z$-direction and the actual wavenumber does not deviate too far from $k_{0}$, the complex envelope $\tilde{u}$ will be significantly smoother than the complex amplitude $\tilde{U}$. However, when the paraxial approximation is applied, the choice of $n_{0}$ affects the error that is introduced by the approximation. For this reason and to ensure that the complex wave amplitude is sufficiently smooth, $n_{0}$ should be a good estimate of the average value in space of the refractive index.

Substitution of equations 3.46, 3.49) and (3.51) in equation (3.44) yields, without any approximation

$$
-\frac{1}{2 k_{0}} \frac{\partial^{2} \tilde{u}}{\partial z^{2}}+j \frac{\partial \tilde{u}}{\partial z}=\frac{1}{2 k_{0}} \nabla_{\perp}^{2} \tilde{u}+\frac{k_{0}}{2}\left(\frac{\tilde{n}^{2}}{n_{0}^{2}}-1\right) \tilde{u},
$$

in which $\nabla_{\perp}^{2}$ is the transverse Laplace operator, which is the Laplace operator excluding the $z$-derivative. The solution $\tilde{u}$ of equation 3.52 is directly related to the solution of the Helmholtz equation by equation (3.49). When introducing the paraxial approximation, this relation will only remain approximately valid. To arrive at the paraxial Helmholtz equation, the first term in equation (3.52), comprising the second derivative in z-direction, is neglected. This approximation is based on the assumption that the wave is paraxial. If the light rays travel at small angles with respect to the optical axis of the laser beam, the gradients in propagation direction will be small. Then, equation (3.52) simplifies to

$$
\frac{\partial \tilde{u}}{\partial z}=-\frac{j}{2 k_{0}} \nabla_{\perp}^{2} \tilde{u}-\frac{j k_{0}}{2}\left(\frac{\tilde{n}^{2}}{n_{0}^{2}}-1\right) \tilde{u} .
$$

Equation (3.53) is the paraxial Helmholtz equation for inhomogeneous media. For homogeneous media, the second term on the right hand side is equal to zero.

Due to the paraxial approximation, the wave propagation becomes unidirectional. Therefore, equation (3.53) represents an initial value problem that can be solved by integrating in the z-direction, which allows for the use of efficient numerical solvers. When implemented in a numerical solver, the switch to unidirectional propagation results in an improvement in computational performance of the order of magnitude of the number of grid points in propagation direction. However, reflections of the wave due to gradients in the refractive index will not be resolved. Therefore, surface reflections were included by reducing the laser power (see section 3.3.1).

Instead of modelling absorption through a complex index of refraction, the wave attenuation was taken into account by adding an additional term to equation (3.53). For an attenuated plane wave travelling along the $z$-direction, the complex envelope is described by

$$
\tilde{u} \propto \sqrt{e^{-\alpha_{\text {tot }}}} .
$$

The additional term in the right hand side of equation (3.53) that is needed to make equation (3.54) a valid solution, is given by

$$
-\frac{\alpha_{\text {tot }}}{2} \tilde{u}
$$


such that the full propagation equation reads

$$
\frac{\partial \tilde{u}}{\partial z}=-\frac{j}{2 k_{0}} \nabla_{\perp}^{2} \tilde{u}-\frac{j k_{0}}{2}\left(\frac{n^{2}}{n_{0}^{2}}-1\right) \tilde{u}-\frac{\alpha_{\text {tot }}}{2} \tilde{u} .
$$

The advantage of this additional term, compared with the use of a complex refractive index, is that the attenuated wave described by equation (3.54) satisfies both equation (3.44) and equation (3.56). Since an attenuated plane wave is a valid solution of the Helmholtz equation and the paraxial approximation should not affect waves that travel parallel to the optical axis, this is a desirable property of the propagation equation.

\subsubsection{Nonlinear Schrödinger equation}

The paraxial Helmholtz equation (3.56) provides an approximate solution of the propagation of a wave through inhomogeneous material, including the effect of refraction and attenuation. However, due to the intense light that is produced by tightly focussed laser pulses, nonlinear effects in the material also have to be taken into account. The main nonlinear effects, for the wavelengths under consideration, are two-photon absorption and self-focussing due to the Kerr effect (see section 2.3). Therefore, in this section, additional terms will be added to the paraxial Helmholtz equation, to include these phenomena. A formal proof that these terms provide a leading order approximation of Maxwell's equations for a nonlinear medium can be found in reference [121].

The Kerr effect is described by a nonlinear refractive index coefficient $n_{2}$ [122]:

$$
n=n_{\text {lin }}+n_{2} I
$$

where $n_{\text {lin }}$ comprises the terms that are not intensity-dependent and $n_{2}$ is the Kerr coefficient. Based on assumption (7) that the variations in the refractive index are small (see section 3.3.2), the approximations $n_{2}^{2}=0$ and $n_{\operatorname{lin}}=n_{0}$ were applied. Substitution of $n=n_{0}+n_{2} I$ and $n_{2}^{2}=0$ in the second term on the right hand side of equation 3.56 yields the following term:

$$
-\frac{j k_{0} n_{2} I \tilde{u}}{n_{0}}
$$

Likewise, two-photon absorption may be included in equation (3.56), by considering an intensity-dependent absorption coefficient $\alpha_{\text {tot }}$. That is,

$$
\alpha_{\text {tot }}=\alpha_{\text {lin }}+\beta I \text {. }
$$

Here, $\beta$ is the two-photon absorption coefficient and $\alpha_{\text {lin }}$ comprises terms regarding the linear absorption across the band gap and the free carrier absorption:

$$
\alpha_{\text {lin }}=\alpha+\sigma N \text {. }
$$

After adding the term given by equation (3.58) to equation 3.56) and substituting equations (3.50), 3.59) and (3.60), the complete propagation equation reads

$$
\frac{\partial \tilde{u}}{\partial z}=-\frac{j}{2 k_{0}} \nabla_{\perp}^{2} \tilde{u}-\frac{j k_{0}}{2}\left(\frac{n_{\text {lin }}^{2}}{n_{0}^{2}}-1\right) \tilde{u}-\frac{\alpha+\sigma N}{2} \tilde{u}-\frac{\beta}{2}|\tilde{u}|^{2} \tilde{u}-\frac{j k_{0} n_{2}|\tilde{u}|^{2} \tilde{u}}{n_{0}} .
$$


This equation is known as a nonlinear Schrödinger-type equation [71, 123] and will be used to simulate the beam propagation inside the material, during all numerical simulations presented in this thesis.

\subsubsection{Collins' diffraction integral}

The paraxial Helmholtz equation that was discussed in section 3.3 .5 is a partial differential equation that is well suited for implementation in a finite difference solver. Since finite difference solvers require the differential equation to be discretised on a fine mesh, they are inefficient to compute the beam path above the surface of the material, including the focussing lens. In the case of circularly symmetric paraxial ray optics, optical components can be described by a ray transfer matrix, also referred to as an ABCD matrix, which alters the position and/or the direction of the rays [85]:

$$
\left[\begin{array}{l}
r_{2} \\
\theta_{2}
\end{array}\right]=\left[\begin{array}{ll}
A & B \\
C & D
\end{array}\right]\left[\begin{array}{l}
r_{1} \\
\theta_{1}
\end{array}\right]
$$

where $r$ is the distance and $\theta$ the angle between the ray and the optical axis. Here, the subscripts one and two refer to the situation before and after a certain optical component.

Ray optics cannot be applied when simulating the formation of laser-induced subsurface modifications, as the effect of diffraction cannot be neglected. Therefore, Collins' diffraction integral [124], which computes the beam propagation including optical components in a single step, was employed. This equation is based on the coefficients of the transfer matrix defined by equation (3.62) and is given by [124, 125]

$$
\tilde{u}_{2}(r)=\frac{2 \pi j}{B \lambda} \exp \left(\frac{-j \pi D r^{2}}{B \lambda}\right) \int_{0}^{\infty} \tilde{u}_{1}\left(r_{1}\right) \exp \left(\frac{-j \pi A r_{1}^{2}}{B \lambda}\right) J_{0}\left(\frac{2 \pi r r_{1}}{B \lambda}\right) r_{1} d r_{1} .
$$

Here, an axisymmetric coordinate system was assumed (see section 3.4.1) and the on-axis phase shift was omitted. Moreover, $J_{o}$ is the Bessel function of order zero of the first kind, $\tilde{u}_{1}$ is the initial complex wave envelope and $\tilde{u}_{2}$ is the envelope after propagation. The back aperture of the focussing objective was taken into account by truncating the beam supplied by the laser source: $\tilde{u}_{1}\left(r_{1}\right)=0$ for $r_{1}>R_{\text {aper }}$, where $R_{\text {aper }}$ is the aperture radius.

To evaluate equation 3.63), the matrix coefficients $A, B$ and $D$ are needed. The focussing objective is modelled as a single thin convex lens using the matrix [85]

$$
\mathbf{M}_{1}=\left[\begin{array}{cc}
1 & 0 \\
\frac{-1}{f} & 1
\end{array}\right]
$$

with $f$ the focal distance. Next, the transmission through the medium between the objective and the sample is described by the matrix [85]

$$
\mathbf{M}_{2}=\left[\begin{array}{cc}
1 & f-\frac{d_{\text {foc }}}{n_{2}} \\
0 & 1
\end{array}\right]
$$


where $d_{\text {foc }}$ is the focus depth inside the sample and $n_{2}$ is the refractive index of the sample. When focussing a laser beam with a Gaussian power density profile, the focus position does not exactly coincide with the geometrical focus [126]. This effect was found to be sufficiently small to neglect it.

The matrix describing the complete beam path up to the sample surface is constructed by matrix multiplications, while placing the first optical component that the laser beam encounters on the right [85], which yields

$$
\mathbf{M}=\mathbf{M}_{2} \mathbf{M}_{1} .
$$

The coefficients of the complete propagation matrix $\mathbf{M}$ provide the required data to evaluate Collins' integral (3.63).

\subsubsection{Gaussian beams and beam quality}

After considering the beam propagation through the material and the optical system above the sample surface, a description of the beam provided by the laser source is required. A solution of the paraxial Helmholtz equation (3.56) for $n=n_{0}$ is [126]:

$$
\tilde{u}=\frac{c}{q(z)} \exp \left(-j k \frac{r^{2}}{2 q(z)}\right) \quad q(z) \in \mathbb{C} \quad c \in \mathbb{R},
$$

where $c$ is a constant which was adapted such that the power of the beam after the aperture of the focussing lens matches the temporal profile of the laser pulse (see section 3.3.1). Choosing $q(z)$ as

$$
\frac{1}{q(z)}=\frac{1}{R(z)}-j \frac{\lambda}{\pi w^{2}(z)},
$$

yields the well-known Gaussian beam that is provided by many laser sources [126]. Here, $w(z)$ is the $1 / e^{2}$ beam radius and $R(z)$ is the radius of curvature of the wavefront. At the waist of the beam, the wavefront radius of curvature is infinite, making $q(z)$ purely imaginary:

$$
\left.q\right|_{\text {waist }}=j \frac{\pi w_{\text {waist }}^{2}}{\lambda}
$$

where $w_{\text {waist }}$ is the waist radius. As $R(z)$ is large for a collimated laser beam, the exact location of the waist is not critical in order to compute the power density profile of the beam after focussing. After the focussing lens, the beam can no longer be described by a Gaussian profile. This is due to the fact that the outer part of the Gaussian distribution is blocked by the aperture of the lens, which introduces appreciable diffraction effects.

Many practical laser sources aim to provide a Gaussian power density profile. However, small deviations from an ideal Gaussian profile are always present. A common method to approximate the effect of these deviations, while still being able to apply the laws for the propagation of Gaussian beams, is the $M^{2}$ factor [127], which is also known as the beam quality. The $M^{2}$ factor is the ratio between the waist-diameter-divergence product of a theoretical Gaussian beam and the one provided by a practical laser source and is expressed as [85]

$$
M^{2}=\frac{\pi}{\lambda} w_{\text {real }} \theta_{\text {real }}
$$


in which the subscript real refers to the actual measured values instead of the values corresponding to a perfect Gaussian beam. To allow the Gaussian beam descriptions that were introduced above to be applied, while compensating for an $M^{2}>1$, the wavelength of the laser beam was adapted by

$$
\hat{\lambda}=\lambda M^{2}
$$

where $\hat{\lambda}$ is the modified wavelength to compensate for the beam quality. This compensation should not be employed when calculating any of the material properties, as the actual wavelength and the corresponding photon energy are unchanged. $M^{2}$ values as supplied by the vendors of the laser sources have been applied when comparing numerical results with experimental data.

\subsubsection{Summary of the beam propagation model}

In sections 3.3.6 3.3.7 and 3.3.8, several equations to describe the power density profile of the laser beam and its propagation through optical components and media have been introduced and discussed. Below, an overview is provided of the equations that were applied to the different sections of the beam path.

Five distinct sections of the beam path can be identified (see figure 3.1). The numbers in the figure correspond to:

1. The laser beam as emitted by the laser source, before entering the focussing objective.

2. The focussing lens including its aperture.

3. Free-space propagation between the lens and the material.

4. Reflection at the interface between the air and the material.

5. The beam propagation through the material.

The following equations were applied to model the behaviour of the beam in these parts:

1. The beam provided by the laser source was described by a Gaussian beam including its beam quality (see section 3.3 .8 ), or by a plane wave.

2-3. The aperture, lens and free spacing propagation were described by Collins' diffraction integral (see section 3.3.7).

4. As the propagation equations do not take reflection losses into account, the power of the beam was reduced by a factor $1-R$, where $R$ is the reflectivity of the material.

5. The beam propagation inside the material was modelled by a nonlinear Schrödinger equation (see section 3.3.6). Reflections at the backside of the material were not considered as these are not expected to have an appreciable effect on the power density profile near the focus. 


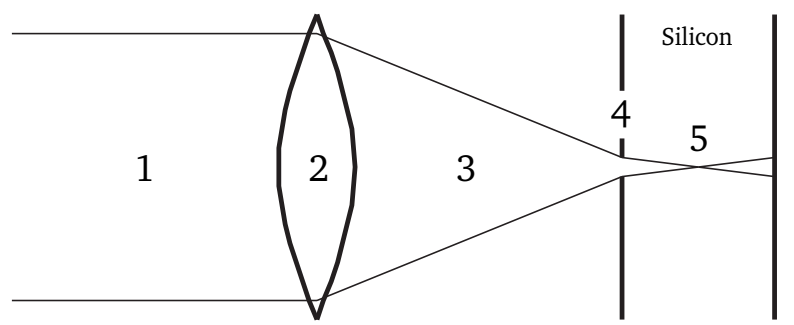

Figure 3.1: Path of the laser beam from the laser source to the bulk of the material that is to be processed.

When computing the radius of the aperture of the objective in the absence of an immersion medium, based on the numerical aperture NA and the focal length $f$, the paraxial approximation has also been applied resulting in

$$
R_{\text {aper }}=f \mathrm{NA} \text {. }
$$

Due to the paraxial approximation, $\lim _{\mathrm{NA} \rightarrow 1} R_{\text {aper }}$ will be a finite value instead of infinity in the non-paraxial case. The ratio between the beam radius and the aperture radius has been matched between the experiments and the simulations. This choice results in a good correspondence between the laser power density profiles in the region near the focus, when comparing the paraxial solution with a solution that satisfies Maxwell's equations (see section 4.2). If $R_{\text {aper }}$ would go to infinity for NA $\rightarrow 1$, infinitely high intensities in the focus would be predicted by the paraxial Gaussian beam solution, which is clearly non-physical.

\subsection{Numerical solvers}

In sections 3.2 and 3.3 , the equations that are part of the two-temperature, mechanical and beam propagation models were described. A combination of existing and custom solvers was employed to solve these equations.

The equations corresponding to the two-temperature model and the mechanical equations (see section 3.2) were solved using the commercial general purpose finite element solver MSC Marc [128] (see section 3.4.2). The computation of the source terms and material properties (see sections 3.2.4 and 3.5), the adaptive time stepping and the post-processing were performed by tailor-made code. The code to extract grid data from the finite element solver was partly based on code supplied by Dillingh [68].

The solvers for Collins' diffraction integral (see section 3.4.4) and the nonlinear Schrödinger equation (see section 3.4.5) were custom developed. The diffraction integral was solved using the quasi fast Hankel transform. Code that is available in the public domain by Sheng and Siegman [129] was used as an example for the implementation. All tailor-made code was written in Fortran 2008 [130].

Three substeps per time step were applied to solve the coupled physical phenomena, resulting in the following process flow for a single time step (see figure 3.2): 
1. Solving the beam propagation based on the distributions of the lattice temperature and carrier density from the previous time step.

2. Solving the conservation equations corresponding to the two-temperature model. These equations compute the distributions of the electron temperature, lattice temperature and carrier density. The material properties are evaluated using the values of the distributions from the previous time step. This also holds for the source terms, excluding the laser intensity.

3. Solving the mechanical equations to obtain the stresses and strains due to thermal expansion, based on the lattice temperature computed during substep (2).

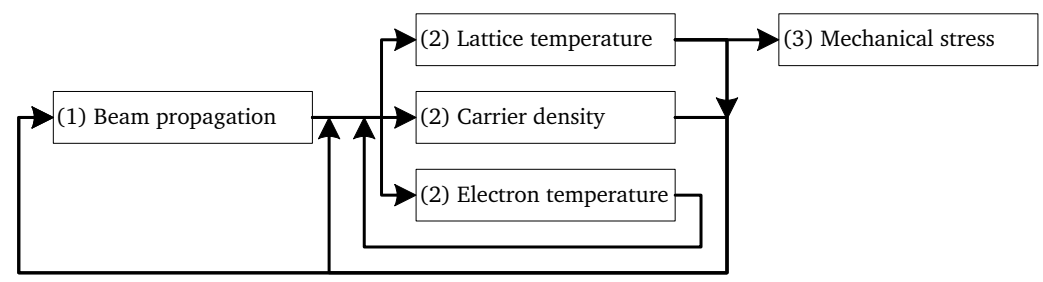

Figure 3.2: Flowchart of the numerical model. Substeps 1-3 are performed consecutively during each time step. When results from the same substep or a subsequent substep are used as input, the input data were obtained from the previous time step.

This approach is referred to as a weak coupling, i.e. the equations corresponding to different physical phenomena are not solved simultaneously. When the time step is sufficiently small, such that the change in any of the distributions between two time steps is limited, weak coupling provides a good approximation of the solutions of the coupled equations.

In this section, the numerical solution methods will be discussed, including the choice of the time step and the design of the computational grid.

\subsubsection{Boundary and initial conditions}

In monocrystalline silicon, material properties may be anisotropic. During experiments on laser melting of silicon using nanosecond pulses, no appreciable differences in processing results have been found for wafers with different crystal orientations [76], which suggests that the anisotropy in optical properties is limited. Therefore, in the simulations, the material has been assumed to be isotropic. This allows for the use of an axisymmetric computational domain, by exploiting the symmetry of the incident laser beam. When selecting an axisymmetric coordinate system, the problem is reduced to two dimensions, namely the radial coordinate $r$ and the coordinate along the optical axis $z$, which are indicated in the cylindrical coordinate system shown in figure 3.3 . Due to the axisymmetry, the solution does not depend on the azimuthal coordinate $\theta$.

The computational domain was chosen sufficiently large in radial direction, such that the material at the outer radius remains near equilibrium within the simulated time. As 


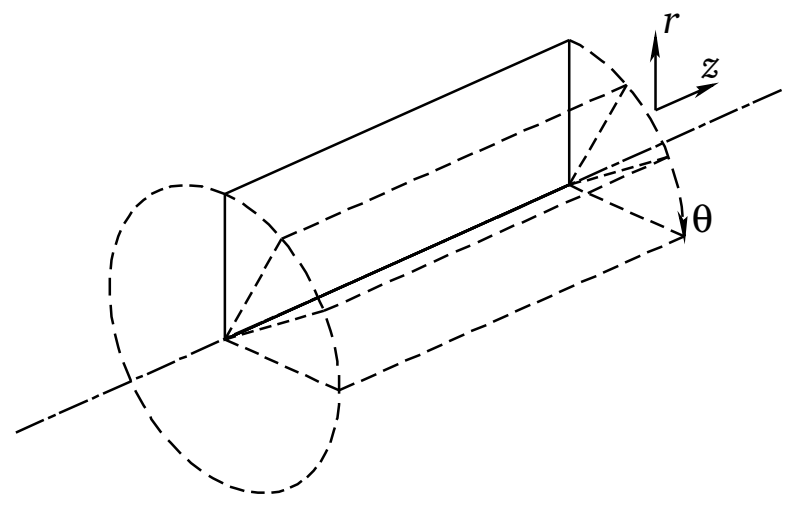

Figure 3.3: Cylindrical coordinate system, since axisymmetric computations were performed, the numerical solutions only depend on the coordinates $r$ and $z$.

long as this condition is satisfied, the size of the computational domain in radial direction may be smaller than the actual size of the sample.

The flux through the symmetry axis is equal to zero and surface recombination, radiative losses and convective losses were neglected. This implies that the following Neumann boundary condition should be applied, for all edges of the lattice temperature, electron temperature and carrier density distributions:

$$
\nabla u(\vec{x}, t) \cdot \vec{n}=0 \quad \forall \vec{x} \in \partial \Omega,
$$

where $u$ is the value of the distribution, which is a function of the location $\vec{x}$ and time $t$. Further, $\vec{n}$ is the outer normal to the edges $\partial \Omega$ of the computational domain $\Omega$. For both the lattice and electron temperature, the initial temperature, at $t=0$, was set to the ambient temperature:

$$
T_{l}(\vec{x}, 0)=T_{e}(\vec{x}, 0)=T_{\mathrm{amb}} .
$$

The initial carrier density, at $t=0$, was set equal to the equilibrium density at ambient temperature $N_{\text {eq }}$.

Regarding the solver for the beam propagation, the complex wave envelope was prescribed at the surface of the sample. The Neumann boundary condition given by equation (3.73) was applied at the symmetry axis and the outer radius of the computational domain. While this does not prevent reflections at the outer radius, a transparent boundary condition was not required since the beam intensity at this radius was verified to be negligible due to the choice of the size of the computational domain.

\subsubsection{Two-temperature model}

As mentioned in section 3.4 the nonlinear finite element solver MSC Marc [128] was used to solve the equations introduced in section 3.2 . These are the conservation equations corresponding to the two-temperature model and the mechanical equations. A detailed description of the solver, including the underlying equations, can be found in reference [128]. 
The conservation equations that belong to the two-temperature model were solved using the transient thermal solver. For the mechanical part, the quasi-static mechanical solver was employed. Quasi-static implies that time-dependent stresses and strains, including mechanical properties that depend on the temperature, can be solved. However, the effect of inertia is neglected.

MSC Marc is an implicit solver that employs a first order accurate backward Euler scheme to discretise the time-derivatives [128]:

$$
\frac{\partial u}{\partial t}=\frac{u_{n}-u_{n-1}}{\Delta t}
$$

in which $u$ is the solution of the conservation equation of either the lattice temperature, electron temperature or carrier density. Further, the indices $n$ and $n-1$ refer to the current and previous time step respectively and $\Delta t$ is the time step. After assembly of the stiffness matrix in the mechanical domain or the capacity and conductivity matrices in the thermal domain, a linear system of equations has to be solved. The incomplete Cholesky-conjugate gradient iterative solution method [131] was selected to solve the system of equations. The solution obtained from the previous time step was used as an initial estimate. As the changes in the solution from time step to time step are relatively small, this solver provides a computational performance benefit over the use of a direct solver, since the initial estimate will be close to the converged solution. Convergence checks were performed to select the maximum allowed residual of the iterative solver.

The capacity matrix was lumped to prevent spurious oscillations while solving the conservation equations [132]. Bi-linear axisymmetric elements were used for both the thermal and mechanical simulations. The thermal expansion coefficients, the constitutive stress-strain matrix, the capacity matrix, the conductivity matrix, the nodal influxes and the initial conditions were defined in custom routines.

Figure 3.4 shows the computational grid used for the two-temperature and mechanical models. For clarity, this figure shows a reduced number of elements. The mesh has been refined in the vicinity of the location of the focus of the laser beam. As the modified volume mainly grows towards the incoming beam during the laser pulse, the focus is located near the edge of the zone comprising the smallest elements. The required size of the elements near the focus, to obtain a converged solution, was $40 \mathrm{~nm}$ in radial direction and $112.5 \mathrm{~nm}$ along the optical axis.

Due to the varying requirements for the design of the grids for the two-temperature and beam propagation models, two different computational grids were used, in order to obtain good overall performance of the numerical code. Since the solution of one of these solvers depends on the field distributions of the other, the grids need to be coupled. At locations where the grids did not overlap, interpolation was employed.

\subsubsection{Time stepping}

When running transient simulations, a suitable time step $\Delta t$ has to be selected. An appropriate choice of the time step depends on the gradients in time of all distributions that are being simulated. Large gradients in time require small time steps, to ensure that the accuracy of the solution is satisfactory. 


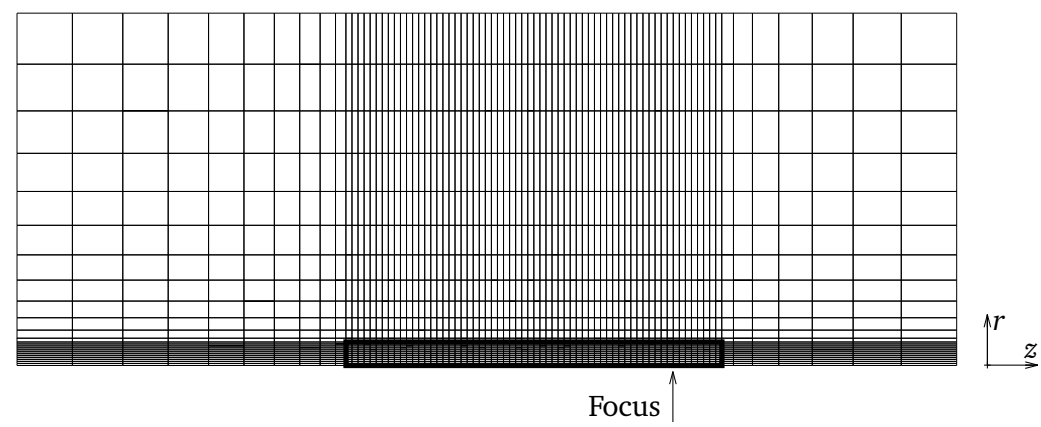

Figure 3.4: Computational grid for the two-temperature and mechanical model. A reduced number of elements have been drawn for visibility purposes. The outer extent of the volume that could possibly contain modified material is plotted using thick lines. The beam propagation direction is from left to right.

As thermal runaways are simulated, the time-gradients can change dramatically during the simulations. Therefore, adaptive time stepping was implemented. The choice of the time step was based on the following criteria:

- A maximum value of the time step of $1 / 1000 \cdot t_{\text {sim }}$.

- A maximum allowed change of the value of the solution at any node of $10 \%$, compared with the previous time increment.

- A maximum change in temperature of $0.1\left(T_{f}-T_{s}\right)$, compared with the previous time step, when the lattice temperature falls within the range in which the latent heat is modelled (see section 3.2.2). If the steps here are too large, the elevated specific heat capacity that accounts for the latent heat could be applied to incorrect temperatures, or be neglected altogether.

It is only possible in retrospect, after the simulation of a time step is complete, to determine if the choice of the time step was correct. Therefore, if the time step was too large, the simulation was restarted at the previous step. All equations that are solved during the simulations use the same step size. Consequently, the distribution that imposes the strongest restraint on the time step determines the selection of $\Delta t$.

The backward Euler scheme that was used to approximate the time-derivatives (see section 3.4.2) is unconditionally stable. Consequently, a stability criterion did not have to be considered when selecting the step size. However, spurious oscillations at the grid boundaries may occur if the ratio between the time step and element size is too small [133]. This is due to the fact that the boundary conditions cannot be properly fulfilled, when the time step does not allow for a meaningful amount of diffusion between neighbouring elements.

To verify whether the criteria that govern the adaptive time stepping were correctly selected, convergence checks were performed. Typical numbers of time steps to simulate a complete laser pulse were between a thousand and ten thousand. 


\subsubsection{Collins' diffraction integral}

The beam propagation above the surface of the material was computed using Collins' diffraction integral (3.63). To evaluate this integral in an axisymmetric coordinate system, it was rewritten as a Hankel transform, allowing it to be solved using an efficient numerical algorithm. Applying the coordinate transform $\rho=r_{1} / B \lambda$ to Collins' integral (3.63) yields

$$
\tilde{u}_{2}(r)=2 \pi j B \lambda \exp \left(\frac{-j \pi D r^{2}}{B \lambda}\right) \int_{0}^{\infty} \tilde{u}_{1}(\rho B \lambda) \exp \left(-j \pi B \lambda A \rho^{2}\right) J_{0}(2 \pi r \rho) \rho d \rho .
$$

The Hankel transform is defined as [134]

$$
g(r)=2 \pi \int_{0}^{\infty} \rho f(\rho) J_{0}(2 \pi r \rho) d \rho,
$$

which allows equation 3.76 to be rewritten as

$$
\tilde{u}_{2}(r)=j B \lambda \exp \left(\frac{-j \pi D r^{2}}{B \lambda}\right) g(r) \text { where } f(\rho)=\tilde{u}_{1}(\rho B \lambda) \exp \left(-j \pi B \lambda A \rho^{2}\right) .
$$

An efficient numerical method to solve the Hankel transform is the quasi fast Hankel transform [134]. This numerical solution method for the Hankel transform starts with the change of variables $\rho=\rho_{0} \exp \left(\alpha_{\exp } x\right)$ and $r=r_{0} \exp \left(\alpha_{\exp } y\right)$, which yields

$$
\hat{g}(y)=\int_{-\infty}^{\infty} \hat{f}(x) \hat{j}(x+y) d x,
$$

where $\hat{f}(x)=\rho f(\rho), \quad \hat{j}(x+y)=2 \pi \alpha_{\exp } \rho r J_{0}(2 \pi \rho r) \quad$ and $\quad \hat{g}(y)=r g(r)$.

Equation (3.79) is identical to the cross-correlation integral except for the absence of the complex conjugate. By defining the discrete sample points

$$
\rho_{n}=\rho_{0} \exp \left(\alpha_{\exp } n\right) \quad \text { and } \quad r_{m}=r_{0} \exp \left(\alpha_{\exp } m\right) \quad n, m \in \mathbb{N}
$$

and the sampled values

$$
f_{n}=\rho_{n} f\left(\rho_{n}\right), \quad g_{m}=r_{m} g\left(r_{m}\right) \quad \text { and } \quad j_{n+m}=2 \pi \alpha_{\exp } \rho_{n} r_{m} J_{0}\left(2 \pi \rho_{n} r_{m}\right),
$$

where $n, m$ are in the range of $0 \ldots N-1, g_{m}$ can be approximated by [134]

$$
g_{m} \approx \sum_{n=0}^{N-1} f_{n} j_{n+m} .
$$

Again, equation (3.82) is similar to the cross-correlation for discrete functions. Equation 3.82) can be evaluated by [134]

$$
\begin{aligned}
& g_{m}=\operatorname{DFT}\left[\operatorname{DFT}\left(f_{m}\right) \times \operatorname{DFT}^{-1}\left(j_{m}\right)\right], \\
& \text { where } m=0 \ldots 2 N-1 \text { with } f_{m}=0 \text { for } m>N-1 .
\end{aligned}
$$


Here, DFT and $\mathrm{DFT}^{-1}$ are forward and backward discrete Fourier transforms respectively. The results for $m=N \ldots 2 N-1$ are aliased [134]. Criteria for the selection of $\alpha_{\text {exp }}, \rho_{0}$ and $r_{0}$ can be found in reference [134].

The discrete Fourier transforms were numerically solved by fast Fourier transforms [135]. Consequently, using the big O notation, equation 3.76 is solved by applying $\mathscr{O}[N \log (N)]$ operations instead of the required $\mathscr{O}\left(N^{2}\right)$ operations when employing direct numerical integration.

In the original publication on the quasi fast Hankel transform [134], the issue of "lower end corrections" is not discussed. When applying equation (3.83), the part of the integral in equation (3.77) between zero and the first discrete sample point is not taken into account. To compensate for this issue, the following lower end correction terms were added to the computed values of $g(r)$ [136]:

$$
f(0) \frac{\rho_{0}}{r} J_{1}\left(2 \pi r \rho_{0}\right)+\left.\frac{d^{2} f}{d \rho^{2}}\right|_{\rho=0} \frac{\rho_{0}^{3}}{4 r}\left[J_{1}\left(2 \pi r \rho_{0}\right)-J_{3}\left(2 \pi r \rho_{0}\right)\right],
$$

where $J_{1}$ and $J_{3}$ are first and third order Bessel functions of the first kind respectively. To evaluate equation (3.84), the second derivative of $f$ was approximated by a second order accurate finite difference scheme.

Since the quasi fast Hankel transform requires an exponential distribution of the sample points, an interpolation step was required when transferring the complex wave envelope to the grid used to compute the beam propagation inside the material.

\subsubsection{Nonlinear Schrödinger equation}

In addition to the solver for the diffraction integral that computes the propagation of the laser beam through the space above the material, a solver for the nonlinear Schrödinger equation (3.61) to simulate the beam propagation inside silicon is required. The solver for this equation is responsible for a large part of the computational effort that is required to model the formation of laser-induced subsurface modifications. Therefore, both a semiimplicit and an explicit finite difference beam propagation method have been evaluated, to select the most efficient numerical solver.

\subsubsection{Finite difference beam propagation methods}

To obtain a finite difference form of the nonlinear Schrödinger equation, the derivatives have to be approximated by discrete equations (see section 3.4.5.2). The two terms including derivatives in this equation are the first order derivative in propagation direction and the transverse Laplace operator.

Practical implementations of finite difference schemes cause two types of errors. The first type of error is the truncation error, which is related to the accuracy of the discrete approximation of the derivatives. The speed of the reduction of the truncation error, when reducing the spacing between the grid points, defines the order of accuracy of the scheme. The second type of error is the algebraic error. This type is related to the finite precision at which real or complex numbers are stored in computer memory, causing rounding errors. 
The most important aspect of finite difference methods is convergence, which implies that the numerical solution approaches the true solution of the continuous partial differential equation when both $\Delta r$ and $\Delta z$ tend to zero [137]. This is not the same as consistency, which only implies that the discrete formulas reproduce the continuous ones when $\Delta r$ and $\Delta z$ go to zero, without ensuring the correctness of the solution [137]. For linear partial differential equations, the Lax equivalence theorem states that for a finite difference scheme to converge, it is a necessary and sufficient condition that the scheme is stable and consistent [138]. For a finite difference beam propagation method to be considered stable, truncation or algebraic errors made during previous steps should not grow while performing additional propagation steps. In the current model, a nonlinear partial differential equation has to be solved to compute the propagation of the laser beam. For nonlinear partial differential equations, the Lax equivalence theorem is not valid and it is hard to obtain formal stability proofs [137]. Therefore, the solver that will be introduced in section 3.4 .5 .3 has been used as a reference implementation, as it is known to provide correct solutions [122].

For the numerical simulations, an axisymmetric coordinate system was used to exploit the rotational symmetry of the laser beam (see section 3.4.1). When employing a uniform grid, the $r$ and z coordinates are related to the discrete indices $(i, j)$ by

$$
\tilde{u}(r, z)=\tilde{u}(i \Delta r, j \Delta z)=\tilde{u}_{i, j} \quad i, j \in \mathbb{N},
$$

in which $\Delta r$ and $\Delta z$ are the grid spacings in radial direction and along the optical axis respectively. In the following sections, the nonlinear Schrödinger equation (3.61), evaluated at location $(i, j)$ on the discrete grid, is rewritten in the following form:

$$
\begin{aligned}
\left.\frac{\partial \tilde{u}}{\partial z}\right|_{(i, j)}= & -\left.\frac{j}{2 k_{0}} \nabla_{\perp}^{2} \tilde{u}\right|_{(i, j)}-\frac{j k_{0}}{2}\left(\frac{n_{\operatorname{lin}}^{2}}{n_{0}^{2}}-1\right) \tilde{u}_{i, j} \\
& -\frac{\alpha+\sigma N}{2} \tilde{u}_{i, j}-\frac{\beta}{2}\left|\tilde{u}_{i, j}\right|^{2} \tilde{u}_{i, j}-\frac{j k_{0} n_{2}\left|\tilde{u}_{i, j}\right|^{2} \tilde{u}_{i, j}}{n_{0}}=\left.c \nabla_{\perp}^{2} \tilde{u}\right|_{(i, j)}+F_{i, j},
\end{aligned}
$$

in which $c$ represents the constant in front of the transverse Laplace operator and $F_{i, j}$ includes all right hand side terms, except for the first term that describes free-space propagation.

\subsubsection{Discretisation of the derivatives}

In this section, discrete approximations of the derivatives in equation 3.86 are derived. First, the transverse Laplace operator will be discretised, including the treatment of the singularity at the symmetry axis of the axisymmetric coordinate system. Next, different discretisations of the first derivative in $z$-direction will be discussed.

Discretisation of the transverse Laplace operator The transverse Laplace operator in an axisymmetric coordinate system is given by

$$
\nabla_{\perp}^{2} \tilde{u}=\frac{\partial^{2} \tilde{u}}{\partial r^{2}}+\frac{1}{r} \frac{\partial \tilde{u}}{\partial r} .
$$


When substituting equation (3.85) in equation (3.87), the transverse Laplace operator evaluated at the discrete grid point $(i, j)$ equals

$$
\left.\nabla_{\perp}^{2} \tilde{u}\right|_{(i, j)}=\left.\frac{\partial^{2} \tilde{u}}{\partial r^{2}}\right|_{(i, j)}+\left.\frac{1}{i \Delta r} \frac{\partial \tilde{u}}{\partial r}\right|_{(i, j)} .
$$

To obtain a discrete form of equation (3.88), the solutions at $r=(i+1) \Delta r$ and $r=(i-1) \Delta r$ are expressed using Taylor expansions in terms of the function values and derivatives at $r=i \Delta r$. The resulting set of equations can then be solved for the derivative that is to be approximated. The Taylor expansions read

$$
\begin{aligned}
& \tilde{u}_{i+1, j}=\tilde{u}_{i, j}+\left.\Delta r \frac{\partial \tilde{u}}{\partial r}\right|_{(i, j)}+\left.\frac{(\Delta r)^{2}}{2 !} \frac{\partial^{2} \tilde{u}}{\partial r^{2}}\right|_{(i, j)}+\left.\frac{(\Delta r)^{3}}{3 !} \frac{\partial^{3} \tilde{u}}{\partial r^{3}}\right|_{(i, j)}+\mathscr{O}\left[(\Delta r)^{4}\right], \\
& \tilde{u}_{i-1, j}=\tilde{u}_{i, j}-\left.\Delta r \frac{\partial \tilde{u}}{\partial r}\right|_{(i, j)}+\left.\frac{(\Delta r)^{2}}{2 !} \frac{\partial^{2} \tilde{u}}{\partial r^{2}}\right|_{(i, j)}-\left.\frac{(\Delta r)^{3}}{3 !} \frac{\partial^{3} \tilde{u}}{\partial r^{3}}\right|_{(i, j)}+\mathscr{O}\left[(\Delta r)^{4}\right],
\end{aligned}
$$

where the term $\mathscr{O}(\ldots)$ indicates the order of magnitude the truncation error. Note that the sign of this term is irrelevant; the truncation error can be a positive or a negative value.

Adding equations 3.89 and 3.90 results in

$$
\tilde{u}_{i+1, j}+\tilde{u}_{i-1, j}=2 \tilde{u}_{i, j}+\left.2 \frac{(\Delta r)^{2}}{2 !} \frac{\partial^{2} \tilde{u}}{\partial r^{2}}\right|_{(i, j)}+\mathscr{O}\left[(\Delta r)^{4}\right] .
$$

Then, the second order derivative is approximated by solving this equation for $\left.\frac{\partial^{2} \tilde{u}}{\partial r^{2}}\right|_{(i, j)}$ :

$$
\begin{aligned}
\left.\frac{\partial^{2} \tilde{u}}{\partial r^{2}}\right|_{(i, j)}=\frac{1}{(\Delta r)^{2}}\left(\tilde{u}_{i+1, j}+\tilde{u}_{i-1, j}-2 \tilde{u}_{i, j}\right. & \left.+\mathscr{O}\left[(\Delta r)^{4}\right]\right) \\
& =\frac{\tilde{u}_{i+1, j}+\tilde{u}_{i-1, j}-2 \tilde{u}_{i, j}}{(\Delta r)^{2}}+\mathscr{O}\left[(\Delta r)^{2}\right] .
\end{aligned}
$$

To approximate the first derivative in equation (3.87), equations 3.89) and 3.90 are subtracted, which yields

$$
\tilde{u}_{i+1, j}-\tilde{u}_{i-1, j}=\left.2 \Delta r \frac{\partial \tilde{u}}{\partial r}\right|_{(i, j)}+\left.2 \frac{(\Delta r)^{3}}{3 !} \frac{\partial^{3} \tilde{u}}{\partial r^{3}}\right|_{(i, j)}+\mathscr{O}\left[(\Delta r)^{4}\right] .
$$

Then, solving for $\left.\frac{\partial \tilde{u}}{\partial r}\right|_{(i, j)}$ gives

$$
\begin{array}{r}
\left.\frac{\partial \tilde{u}}{\partial r}\right|_{(i, j)}=\frac{1}{2 \Delta r}\left(\tilde{u}_{i+1, j}-\tilde{u}_{i-1, j}-\left.2 \frac{(\Delta r)^{3}}{3 !} \frac{\partial^{3} \tilde{u}}{\partial r^{3}}\right|_{(i, j)}+\mathscr{O}\left[(\Delta r)^{4}\right]\right) \\
=\frac{\tilde{u}_{i+1, j}-\tilde{u}_{i-1, j}}{2 \Delta r}+\mathscr{O}\left[(\Delta r)^{2}\right] .
\end{array}
$$


Finally, substituting equations 3.92 and 3.94 in equation 3.88, yields a second order accurate discrete approximation of the transverse Laplace operator in an axisymmetric coordinate system:

$$
\begin{aligned}
\left.\nabla_{\perp}^{2} \tilde{u}\right|_{(i, j)} & =\left.\frac{\partial^{2} \tilde{u}}{\partial r^{2}}\right|_{(i, j)}+\left.\frac{1}{i \Delta r} \frac{\partial \tilde{u}}{\partial r}\right|_{(i, j)} \\
& =\frac{\tilde{u}_{i+1, j}+\tilde{u}_{i-1, j}-2 \tilde{u}_{i, j}}{(\Delta r)^{2}}+\frac{1}{i \Delta r} \frac{\tilde{u}_{i+1, j}-\tilde{u}_{i-1, j}}{2 \Delta r}+\mathscr{O}\left[(\Delta r)^{2}\right] \\
& =\frac{1}{(\Delta r)^{2}}\left[\left(1-\frac{1}{2 i}\right) \tilde{u}_{i-1, j}+\left(1+\frac{1}{2 i}\right) \tilde{u}_{i+1, j}-2 \tilde{u}_{i, j}\right]+\mathscr{O}\left[(\Delta r)^{2}\right] .
\end{aligned}
$$

Second order accurate means that the truncation error scales with $(\Delta r)^{2}$.

Singularity and pole condition Equation (3.88) is singular for $r=0$. Therefore, a pole condition is required. When an axisymmetric beam is solved in a Cartesian coordinate system, this singularity is not present. Consequently, the singularity for $r=0$ is a coordinate singularity and is not related to the physics of the problem.

To solve the issue of the singularity, the transverse Laplace operator at $r=0$ has been replaced by its limit for $r \rightarrow 0$ [139]. This limit is given by

$$
\lim _{r \rightarrow 0} \nabla_{\perp}^{2} \tilde{u}=\lim _{r \rightarrow 0}\left(\frac{\partial^{2} \tilde{u}}{\partial r^{2}}+\frac{1}{r} \frac{\partial \tilde{u}}{\partial r}\right)=\left.\frac{\partial^{2} \tilde{u}}{\partial r^{2}}\right|_{r=0}+\lim _{r \rightarrow 0}\left(\frac{1}{r} \frac{\partial \tilde{u}}{\partial r}\right) .
$$

Since an axisymmetric coordinate system is employed, $\lim _{r \rightarrow 0} \frac{\partial \tilde{u}}{\partial r}=0$. Therefore, the second term in the right hand side of equation (3.96) can be evaluated using l'Hôpital as

$$
\lim _{r \rightarrow 0}\left(\frac{1}{r} \frac{\partial \tilde{u}}{\partial r}\right)=\lim _{r \rightarrow 0} \frac{\frac{\partial}{\partial r} \frac{\partial \tilde{u}}{\partial r}}{\frac{\partial}{\partial r} r}=\lim _{r \rightarrow 0} \frac{\partial^{2} \tilde{u}}{\partial r^{2}}=\left.\frac{\partial^{2} \tilde{u}}{\partial r^{2}}\right|_{r=0} .
$$

Substituting equations 3.85, 3.92 and 3.97 in equation 3.96, yields the following discrete approximation of the limit of the transverse Laplace operator for $r \rightarrow 0$ :

$$
\lim _{r \rightarrow 0} \nabla_{\perp}^{2} \tilde{u}=2 \frac{\tilde{u}_{1, j}+\tilde{u}_{-1, j}-2 \tilde{u}_{0, j}}{(\Delta r)^{2}}+\mathscr{O}\left[(\Delta r)^{2}\right] .
$$

Finally, the fact that $r=0$ is a symmetry axis implies that $\tilde{u}_{1, j}=\tilde{u}_{-1, j}$, which results in

$$
\lim _{r \rightarrow 0} \nabla_{\perp}^{2} \tilde{u}=4 \frac{\tilde{u}_{1, j}-\tilde{u}_{0, j}}{(\Delta r)^{2}}+\mathscr{O}\left[(\Delta r)^{2}\right]
$$

Discretisation of the first derivative in propagation direction Apart from the transverse Laplace operator, the first derivative in $z$-direction in equation (3.86) has to be discretised. Three different discretisations of this derivative will be applied for the finite difference schemes that are discussed in the following sections. Equivalent to the discrete 
form of the first derivative in $r$-direction (3.94), the $z$-derivative can be discretised by a central difference scheme:

$$
\left.\frac{\partial \tilde{u}}{\partial z}\right|_{(i, j)}=\frac{\tilde{u}_{i, j+1}-\tilde{u}_{i, j-1}}{2 \Delta z}+\mathscr{O}\left[(\Delta z)^{2}\right] .
$$

Similarly, a central difference scheme to compute the first derivative between two grid points is

$$
\left.\frac{\partial \tilde{u}}{\partial z}\right|_{\left(i, j+\frac{1}{2}\right)}=\frac{\tilde{u}_{i, j+1}-\tilde{u}_{i, j}}{\Delta z}+\mathscr{O}\left[(\Delta z)^{2}\right] .
$$

Finally, a forward difference scheme is derived based on the following Taylor expansion:

$$
\tilde{u}_{i, j+1}=\tilde{u}_{i, j}+\left.\Delta z \frac{\partial \tilde{u}}{\partial z}\right|_{(i, j)}+\mathscr{O}\left[(\Delta z)^{2}\right] .
$$

Then, solving for $\left.\frac{\partial \tilde{u}}{\partial z}\right|_{(i, j)}$ yields

$$
\left.\frac{\partial \tilde{u}}{\partial z}\right|_{(i, j)}=\frac{1}{\Delta z}\left(\tilde{u}_{i, j+1}-\tilde{u}_{i, j}+\mathscr{O}\left[(\Delta z)^{2}\right]\right)=\frac{\tilde{u}_{i, j+1}-\tilde{u}_{i, j}}{\Delta z}+\mathscr{O}(\Delta z) .
$$

This scheme is known as the forward Euler scheme [137]. Since the truncation error is of order $\Delta z$, the forward Euler scheme is only first order accurate.

\subsubsection{Crank-Nicolson method}

An unconditionally stable finite difference scheme, for the case of free-space propagation, is given by substituting equation (3.101) in equation 3.86 and taking the average of equation (3.95) at grid points $j$ and $j+1$ to approximate the transverse Laplace operator at point $j+1 / 2$ :

$$
\begin{aligned}
\frac{\tilde{u}_{i, j+1}-\tilde{u}_{i, j}}{\Delta z}+\epsilon & =\frac{1}{2} c \frac{1}{(\Delta r)^{2}}\left[\left(1-\frac{1}{2 i}\right)\left(\tilde{u}_{i-1, j}+\tilde{u}_{i-1, j+1}\right)+\right. \\
& \left.\left(1+\frac{1}{2 i}\right)\left(\tilde{u}_{i+1, j}+\tilde{u}_{i+1, j+1}\right)-2\left(\tilde{u}_{i, j}+\tilde{u}_{i, j+1}\right)\right]+\mathscr{O}\left[(\Delta r)^{2}\right]+F .
\end{aligned}
$$

This scheme is known as the Crank-Nicolson scheme [140]. The truncation error $\epsilon$ is $\mathscr{O}\left[(\Delta z)^{2}\right]$ for $F=0$, which holds for free-space propagation. This is an implicit scheme, as the solutions at locations $(i+1, j+1)$ and $(i-1, j+1)$ are required to compute the solution at $(i, j+1)$. Consequently, a system of equations has to be solved to obtain the solutions at the discrete grid points corresponding to index $j+1$.

Discretisation of the source terms The discretisation of the combination of source terms $F$ (see section 3.4.5.1), which is required to include the terms that describe the propagation through a material instead of free space, is not considered in equation (3.104). Since $F$ contains nonlinear terms, this implies that an implicit scheme requires a system of 
nonlinear equations to be solved. Solving such a system of equations is computationally far more expensive than solving a linear system of equations. Consequently, the source terms have been evaluated in an explicit manner. Therefore, the unconditional stability of the Crank-Nicolson scheme is lost [122].

The linear terms in $F$ that depend on the temperature or carrier density were also treated in an explicit manner. Consequently, for a fixed $\Delta z$, the system of equations can be solved for each propagation step by a vector addition and two multiplications of vectors with precomputed matrices [122]. To fill one of these matrices, a matrix inversion is required, which was performed numerically using LAPACK [141].

The remaining question is what explicit scheme should be used to discretise the source terms $F$. A straightforward choice would be to apply forward Euler 3.103 : $F=F_{i, j}$. A disadvantage of the forward Euler scheme is that it is only first order accurate, while the Crank-Nicolson scheme is second order accurate. Therefore the truncation error $\epsilon$ in equation (3.104) becomes $\mathscr{O}(\Delta z)$. An alternative explicit forward difference scheme is the second order accurate two-step Adams-Bashforth scheme [142], which is given by

$$
F=\frac{3}{2} F_{i, j}-\frac{1}{2} F_{i, j-1}
$$

resulting in a truncation error $\epsilon$ of $\mathscr{O}\left[(\Delta z)^{2}\right]$, which is identical to the free-space propagation case. No significant changes in the simulation results were found when employing the second order accurate Adams-Bashforth scheme compared with the forward Euler scheme. This is due to the fact that stability and not accuracy was the limiting factor for the choice of the grid spacing along the optical axis (see section 3.4.5.5). Therefore, the forward Euler scheme was selected for its simplicity.

The semi-implicit Crank-Nicolson scheme that is described in this section is known to provide correct solutions, when solving nonlinear Schrödinger-type equations including two-photon absorption and the Kerr effect [122]. Therefore, solutions obtained using this solver have been used as reference solutions.

\subsubsection{Explicit finite difference beam propagation method}

In addition to the implicit Crank-Nicolson method, an explicit scheme that is conditionally stable, known as the explicit finite difference beam propagation method (EFD-BPM) [143], was considered. The EFD-BPM is based on substituting equations 3.95) and (3.100) in equation 3.86):

$$
\begin{aligned}
& \frac{\tilde{u}_{i, j+1}-\tilde{u}_{i, j-1}}{2 \Delta z}+\mathscr{O}\left[(\Delta z)^{2}\right]= \\
& \quad c \frac{1}{(\Delta r)^{2}}\left[\left(1-\frac{1}{2 i}\right) \tilde{u}_{i-1, j}+\left(1+\frac{1}{2 i}\right) \tilde{u}_{i+1, j}-2 \tilde{u}_{i, j}\right]+\mathscr{O}\left[(\Delta r)^{2}\right]+F_{i, j} .
\end{aligned}
$$

This is an explicit scheme, as the right hand side is evaluated based on data from the previous grid layer in propagation direction. Consequently, $\tilde{u}_{i, j+1}$ can be computed without solving a system of equations. The grid points used to approximate the $z$-derivative and transverse Laplacian and the function $F$ do not overlap during a propagation step. 
Figure 3.5 shows the grid points that are used for the discretisation of the different terms in equation 3.106 .
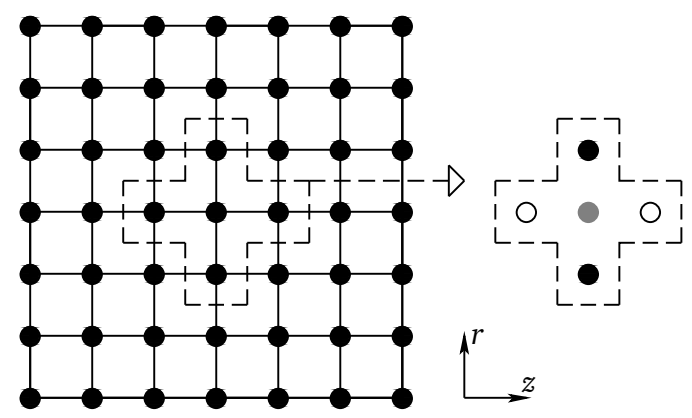

Transverse Laplacian

and source terms

oz-derivative

- Transverse Laplacian

Figure 3.5: Grid points used for the discretisation of the terms in the nonlinear Schrödinger equation, when applying the explicit finite difference beam propagation method.

Since the EFD-BPM is a two-step scheme, a single-step finite difference scheme is required to start the computation at the surface of the material, where the initial complex wave envelope $\tilde{u}$ is defined. To derive an explicit forward Euler single-step scheme, equations (3.95) and (3.103) are substituted in equation 3.86) which gives

$$
\begin{aligned}
& \frac{\tilde{u}_{i, j+1}-\tilde{u}_{i, j}}{\Delta z}+\mathscr{O}(\Delta z)= \\
& \quad c \frac{1}{(\Delta r)^{2}}\left[\left(1-\frac{1}{2 i}\right) \tilde{u}_{i-1, j}+\left(1+\frac{1}{2 i}\right) \tilde{u}_{i+1, j}-2 \tilde{u}_{i, j}\right]+\mathscr{O}\left[(\Delta r)^{2}\right]+F_{i, j} .
\end{aligned}
$$

The forward Euler method is unconditionally unstable for the current problem [143]. This means that a growth rate of truncation and/or algebraic errors smaller than one cannot be obtained for every possible error, regardless of the choice of the grid spacing. However, for a single propagation step at a boundary, this does not constitute a problem. As forward Euler is only first order accurate, an additional half-step was employed for improved accuracy.

In contrast to the forward Euler method, the EFD-BPM scheme is stable for a sufficiently small propagation step [143]. For the case of an unattenuated wave and excluding nonlinear effects, the stability criterion is given by [143]

$$
\Delta z<\frac{2 k_{0}}{\frac{4}{(\Delta r)^{2}}+\frac{k_{0}^{2}}{n_{0}^{2}}\left|n_{\operatorname{lin}}^{2}-n_{0}^{2}\right|_{\max }},
$$

where the subscript max refers to the maximum value when considering all discrete grid points.

Computational mode In the presence of nonlinearities, tests using the EFD-BPM showed that it was unstable when simulating certain laser processing conditions, due to the presence of an undamped computational mode. A computational mode is a solution allowed by the discrete scheme that is non-physical. The computational mode was found 
to have a period of $2 \Delta z$, i.e. it was related to the complex wave envelope at the odd and even grid points in propagation direction drifting away from each other. This is a consequence of the use of a two-step scheme. It can be observed in figure 3.5 that the odd and even grid points are only coupled by the Laplace operator and the source terms. For the discretisation of the $z$-derivative, the odd and even grid points are uncoupled.

Similar issues have been observed when solving hyperbolic advection equations using a leap frog scheme [144], in which the computational mode affects the time integration. Two solutions which are known to be suitable to damp the computational mode in advection equations are [144]:

- To apply a Robert-Asselin filter [145].

- To periodically restart the two-step scheme using a single-step scheme, thereby discarding the solution corresponding to the odd or even grid points.

The Robert-Asselin filter is defined as [145]

$$
\hat{u}_{i, j}=\tilde{u}_{i, j}+\frac{v}{2}\left(\tilde{u}_{i, j-1}-2 \tilde{u}_{i, j}+\tilde{u}_{i, j+1}\right),
$$

where $\hat{u}$ is the complex wave envelope after filtering and $v$ is the Robert-Asselin filter parameter, which is a value in the range of $[0,1]$. For $v=0$, the filter is disabled. The required value of $v$ to effectively suppress the computational mode is problem-dependent.

Numerical experiments showed that both solutions were suitable to suppress the computational mode induced by the scheme given by equation (3.106). However, the solution to periodically restart the EFD-BPM was preferred as it does not require artificial changes to the complex wave amplitude. A restart every fifty steps was found to be a satisfactory choice for all laser processing conditions that were considered. Equivalent to the initial excitation of the two-step scheme at the boundary of the computational domain, forward Euler was selected for the restarts. Also for the restarts, an extra half-step was employed for improved accuracy.

To test the correctness of the EFD-BPM combined with periodic restarts using the forward Euler scheme, a comparison with the Crank-Nicolson scheme was performed. One of the aspects that had to be tested was the stability. Since the finite difference scheme that was used for the periodic restarts of the two-step scheme is unstable when used on its own, the question is how this will affect the stability of the complete solver. When comparing the results of the Crank-Nicolson method with the EFD-BPM including periodic restarts, no significant differences were found between the obtained solutions.

\subsubsection{Selection of the finite difference scheme}

Both the Crank-Nicolson (see section 3.4.5.3) and the explicit finite difference beam propagation method (see section 3.4.5.4) were found to be suitable to solve the nonlinear Schrödinger equation. Therefore, the selection of the finite difference scheme was based on the computational performance. The performance of the fully explicit method was found to be better by approximately a factor hundred, for the specific problem that is considered in this work. Therefore, the EFD-BPM was selected to solve equation (3.61). 
Even in the absence of nonlinear terms, resulting in unconditional stability of the CrankNicolson method, the EFD-BPM was found to have a performance benefit. In spite of the fact that the EFD-BPM requires small steps in propagation direction to satisfy the stability criterion, the speed increase due to the reduced computational effort per step was found to outweigh the benefit of the reduced number of steps when using the Crank-Nicolson method. In the presence of nonlinear effects, the speed advantage of the EFD-BPM becomes even larger. For nonlinear problems, the step size in propagation direction has to be reduced when employing the Crank-Nicolson method, due to the conditional stability criterion imposed by the explicit treatment of the nonlinear terms.

The stability criterion was found to be the limiting factor regarding the selection of the grid spacing along the optical axis. The grid spacing in radial direction was governed by the accuracy and was selected sufficiently small to obtain a converged solution. The spacings between the grid points in radial direction and along the optical axis were $\Delta r=40 \mathrm{~nm}$ and $\Delta z=5 \mathrm{~nm}$ respectively.

\subsection{Material properties}

The material that was selected for the simulations is intrinsic monocrystalline silicon. The parameters that were used are listed in table 3.1. Most data is for the solid phase. For the liquid and gaseous phase, only data regarding the thermodynamic properties were included. Since melting and resolidification was considered to induce a subsurface modification, the physics that occur after melting will not lead to a different answer to the question whether a modification was formed (see section 3.2.6). Therefore, the primary goal of the model, which is to select suitable processing conditions, can be satisfied.

If available, material data that was experimentally validated was preferred. Such validations generally do not cover the full range of temperatures and carrier densities that are encountered during the laser-material interaction. When the range of the experimental validation is exceeded, the accuracy of the material data is unknown. Consequently, the uncertainties in the numerical simulations cannot be quantified. Therefore, the simulation results will be compared with experimental data in chapter 6 .

\subsection{Summary}

In this chapter, a numerical model to predict suitable processing conditions for the formation of subsurface modifications in silicon has been proposed. The model is based on a twotemperature model to simulate the response of the material to the absorbed energy, a beam propagation model to simulate the propagation of the laser light and a mechanical model to predict stresses due to thermal expansion. Equations and solvers to efficiently simulate the beam propagation inside and outside the material were described, including their relation to Maxwell's equations. An explicit finite difference solver was selected to compute the beam propagation inside the material, while a solver based on the quasi fast Hankel transform was chosen to simulate the rest of the beam path. In chapter 4 , the results of the numerical simulations will be presented. 


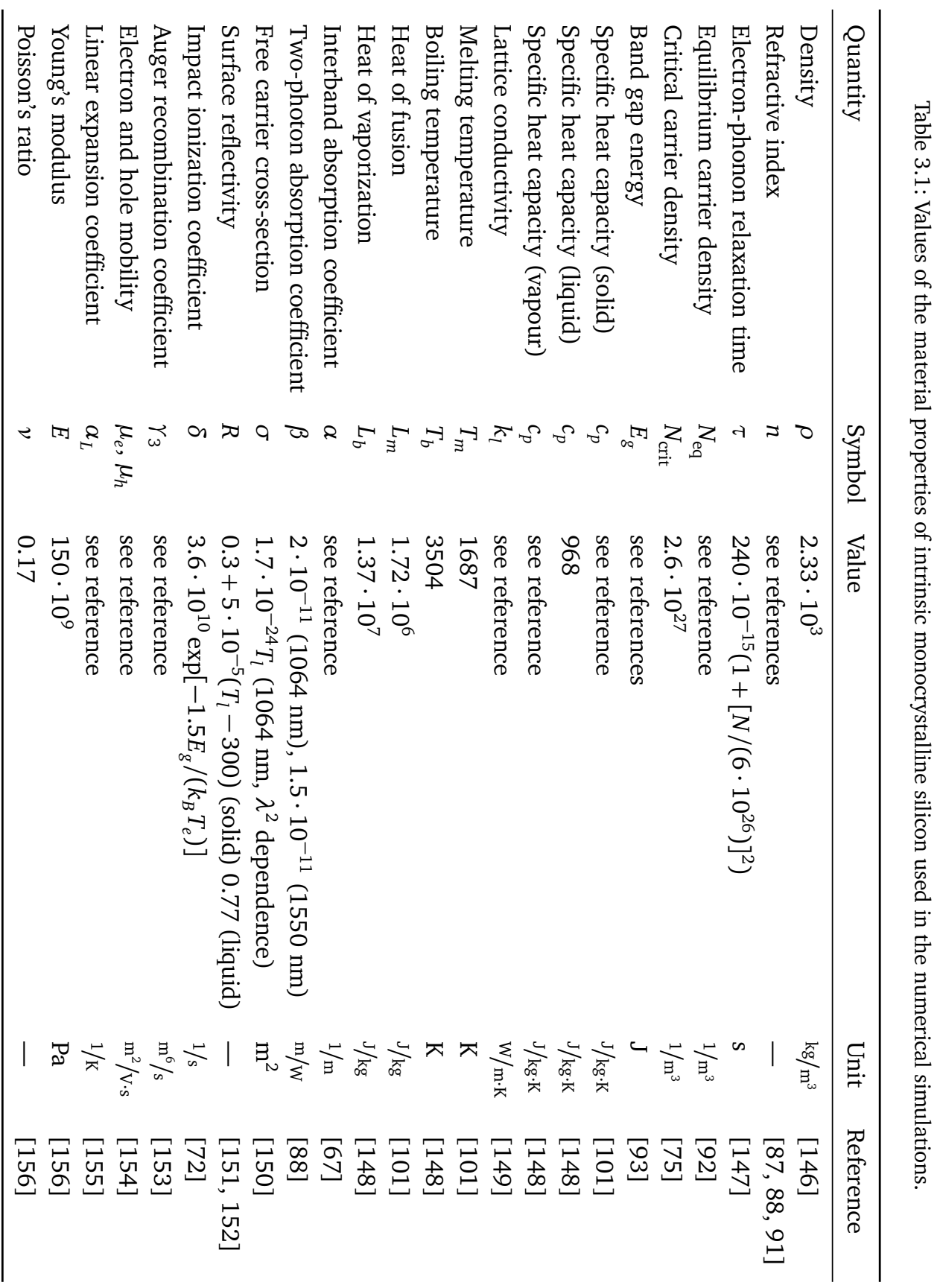




\section{Simulation results}

This chapter* describes the simulation results that were obtained from the numerical model that was proposed in chapter 3. Parameter studies were performed to predict the required processing conditions for the formation of subsurface modifications in silicon. Additionally, the impact of variations in the laser parameters, material properties and initial conditions on the shape of the subsurface modifications was investigated.

\subsection{Introduction}

Two wavelengths were considered during the numerical simulations, namely 1064 and $1550 \mathrm{~nm}$ (see section 2.8). Both wavelengths enable two-photon absorption at sufficiently high light intensities. However, at room temperature, the $1064 \mathrm{~nm}$ wavelength also results in a limited amount of single-photon interband absorption. Consequently, a thermal runaway can be triggered by exploiting the temperature-dependence of the linear absorption coefficient (see section 2.4). Linear interband absorption can also occur at $1550 \mathrm{~nm}$, but only at lattice temperatures beyond $1000 \mathrm{~K}$, when the band gap has shrunken considerably. Only single-pulse modifications were considered. Therefore, the simulation results are not representative for overlapping laser pulses, as used during the experiments in references [64,66].

The process parameters that were employed for the numerical simulations are listed in table 4.1. The numerical aperture of the focussing objective was set to 0.7 , to match the objective that was selected for the experimental set-up (see section 5.4). Although simulations with picosecond pulses were performed, most of the results that are presented for the 1064 and $1550 \mathrm{~nm}$ wavelengths are based on pulse durations of 5 and 1 ns respectively. These correspond to near-optimal processing conditions (see section 4.5.2). Optimal is defined as providing the largest modifications for a fixed pulse energy. Simulating the optimum pulse duration for each wavelength was preferred over selecting a fixed pulse duration, to obtain a fair comparison between the performance of both wavelengths.

In the next section, the laser beam profile under equilibrium conditions will be considered, including a comparison with a solution satisfying Maxwell's equations to verify the correctness of the beam propagation model. Then, figures showing the distributions of relevant physical quantities during the laser pulse will be presented. After that, the modification mechanisms that are responsible for the formation of subsurface modifications will be evaluated. Finally, the results of parameter studies are discussed. During these

\footnotetext{
*Simulation results have been published in Applied Physics A: Materials Science \& Processing, 144(4):1135-1143, 2014 (P. C. Verburg, G. R. B. E. Römer, and A. J. Huis in 't Veld), Proceedings of the 14th International Symposium on Laser Precision Microfabrication, 2013 (P. C. Verburg, G. R. B. E. Römer, and A. J. Huis in 't Veld) and Proceedings of the 13th International Symposium on Laser Precision Microfabrication, 2012 (P. C. Verburg, G. R. B. E. Römer, G. H. M. Knippels, J. Betz, and A. J. Huis in ’t Veld).
} 
studies, the influence of the pulse duration, pulse energy, wavelength, initial temperature, doping concentration and focus depth on the geometry of the subsurface modifications has been investigated.

Table 4.1: Process parameters used for the numerical simulations. The pulse duration is defined as the full duration at half maximum (FDHM).

\begin{tabular}{ll}
\hline Property & Value \\
\hline Wavelength & 1064 or $1550 \mathrm{~nm}$ \\
Pulse duration & $50 \mathrm{ps}-500 \mathrm{~ns}$ \\
Pulse energy & $0.5-2 \mu \mathrm{J}$ \\
Numerical aperture of the objective & 0.7 \\
Spatial power density profile of the beam & Gaussian $\left(M^{2}=1\right)$ \\
Temporal profile of the laser power & Gaussian \\
Beam diameter $\left(1 / e^{2}\right)$ & $80 \%$ of aperture \\
Wafer thickness & $160 \mu \mathrm{m}$ \\
Focus depth & $100 \mu \mathrm{m}$ \\
Ambient temperature & $293 \mathrm{~K}$ \\
\hline
\end{tabular}

\subsection{Laser beam profiles under equilibrium conditions}

The spatial profile of the laser beam that is emitted by the laser source influences the distribution of the laser intensity inside silicon, after the beam has been focussed by a microscope objective. The scaling of the beam with respect to the back aperture of the objective is of importance. A too narrow beam results in a larger than optimal spot size in the focal plane. However, a too wide beam induces power losses and aperture diffraction effects.

To assess the effect of the laser beam profile on the subsurface intensity distribution of the beam, three different profiles have been simulated for a wavelength of $1064 \mathrm{~nm}$ :

1. A Gaussian beam with $99 \%$ of the power contained within the back aperture of the objective (see figure 4.1).

2. A Gaussian beam with the $1 / e^{2}$ beam width filling $80 \%$ of the back aperture of the objective (see figure 4.2)

3. A plane wave that fills the back aperture of the objective (see figure 4.3). A similar result may be obtained by strongly overfilling the aperture with a Gaussian beam.

The laser intensity distributions were computed for silicon at room temperature, without the presence of photo-ionized carriers. Consequently, only a small amount of linear interband absorption and free carrier absorption occurs, resulting in a slight asymmetry of the laser intensity distribution around the focus. 
To establish the beam diameter that corresponds to profile (1), the aperture losses have to be evaluated. The power which is transmitted through a circular aperture is given by [126]

$$
P_{\text {trans }}=P\left[1-\exp \left(\frac{-2 R_{\text {aper }}^{2}}{w^{2}}\right)\right] .
$$

To obtain a transmission of $P_{\text {trans }}=0.99 P$, the $1 / e^{2}$ beam radius $w$ at the focussing lens should be chosen as follows:

$$
w=R_{\text {aper }} \sqrt{1 / \ln (10)} \approx 0.66 R_{\text {aper }}
$$

where $R_{\text {aper }}$ is the aperture radius. Consequently, profile (1) corresponds to a smaller laser beam radius than profile (2).

In figures $4.1,4.2$ and 4.3 , the intensity profiles corresponding to the above mentioned profiles have been plotted. A logarithmic scale is used, to ensure that the local maxima and minima due to the effect of aperture diffraction are clearly visible. The intensity is given in an arbitrary unit; the peak intensity that was obtained during each simulation was set to one.

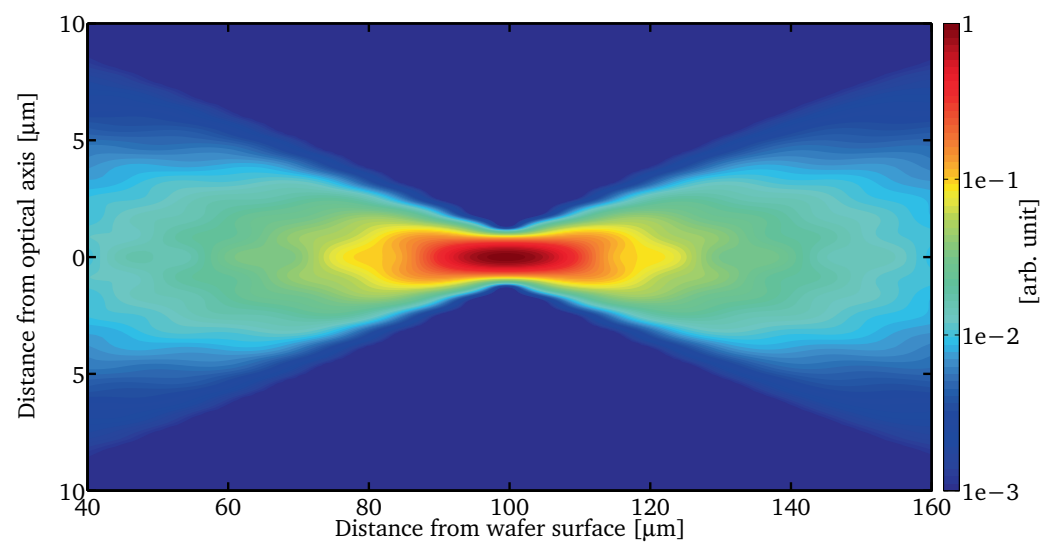

Figure 4.1: Cross-section of the laser intensity distribution [arbitrary unit] in the bulk of silicon. The laser beam at the back of the objective is Gaussian with $99 \%$ percent of the power contained within the aperture. Wavelength: $1064 \mathrm{~nm}$. The beam propagation direction is from left to right. Focal diameter: $1.60 \mu \mathrm{m}\left(1 / e^{2}\right)$.

The laser intensity distributions show that filling the back aperture with a plane wave provides the smallest focal spot, with a $1 / e^{2}$ diameter of $1.20 \mu \mathrm{m}$ (see figure 4.3). The resulting intensity distribution is known as the Airy pattern [157]. When underfilling the objective with a Gaussian beam (see figures 4.1 and 4.2), the effect of diffraction from the aperture gradually disappears when decreasing the laser beam diameter. On the other hand, the size of the focal spot also increases. The diameters of the focal spot for beam profiles (1) and (2) are $1.60 \mu \mathrm{m}$ and $1.44 \mu \mathrm{m}$ respectively. For both the experiments and the numerical simulations, beam profile (2) was selected, as it provides a good compromise between obtaining a small spot size and preventing power losses and diffraction effects due to the aperture of the focussing objective. 


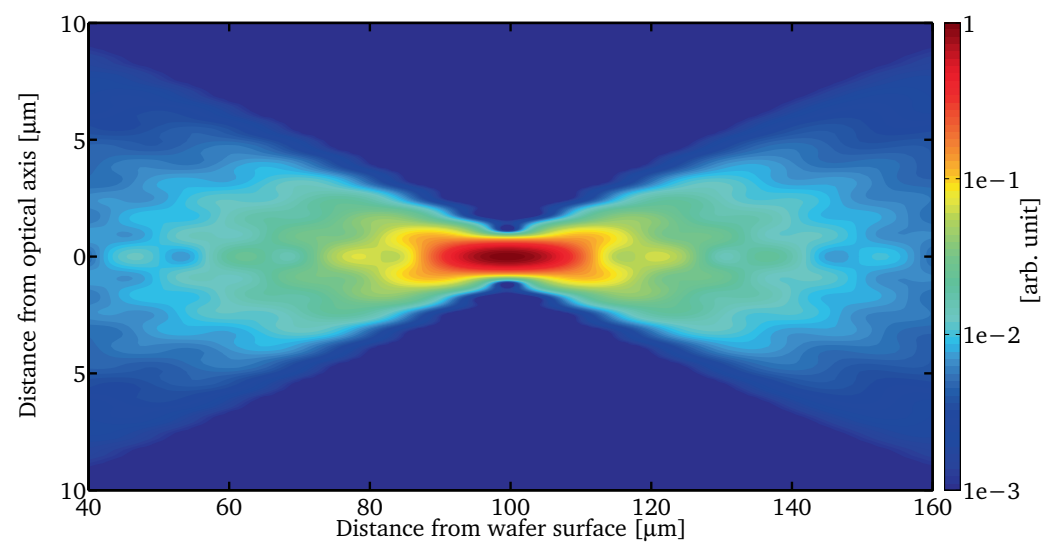

Figure 4.2: Cross-section of the laser intensity distribution [arbitrary unit] in the bulk of silicon. The laser beam at the back of the objective is Gaussian with the $1 / e^{2}$ beam width filling $80 \%$ of the aperture. Wavelength: $1064 \mathrm{~nm}$. The beam propagation direction is from left to right. Focal diameter: $1.44 \mu \mathrm{m}\left(1 / e^{2}\right)$.

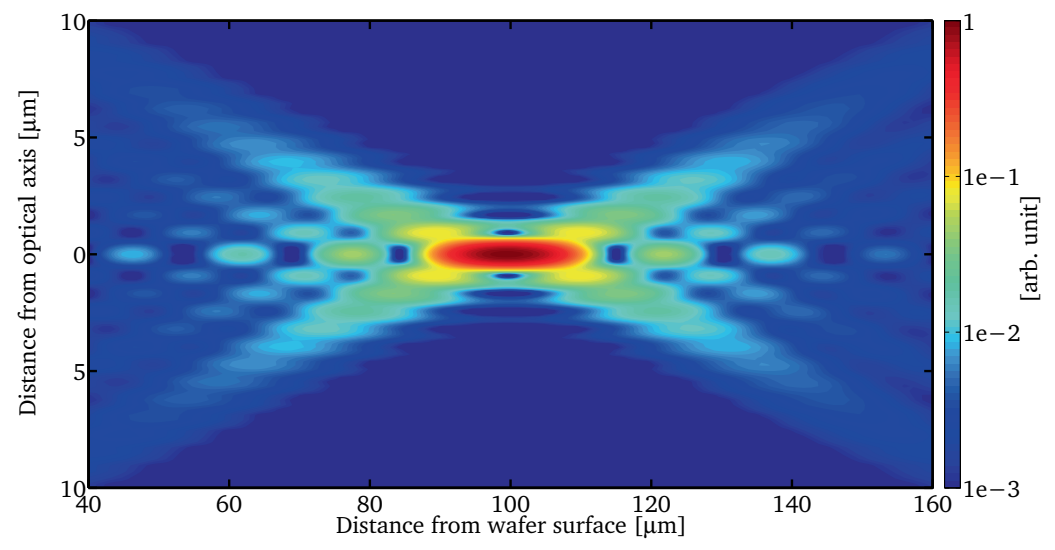

Figure 4.3: Cross-section of the laser intensity distribution [arbitrary unit] in the bulk of silicon. The laser beam at the back of the objective is a plane wave. Wavelength: $1064 \mathrm{~nm}$. This intensity pattern in known as the Airy pattern [157]. The beam propagation direction is from left to right. Focal diameter: $1.20 \mu \mathrm{m}\left(1 / e^{2}\right)$. 
Verification of the beam propagation model Beam profiles (1) and (3) have been compared with analytical descriptions of a Gaussian beam and the Airy pattern, to verify the correctness of the numerical code.

The $1 / e^{2}$ waist diameter $d_{0}$ of a Gaussian beam can be approximated by [126]

$$
d_{0} \approx \frac{2 f \lambda_{0}}{\pi w}
$$

In this analytical expression, the effect of aperture diffraction has been neglected, since $99 \%$ of the power is transmitted through the aperture for beam profile (1). To replace the focal length $f$ in equation (4.3) by the numerical aperture and aperture radius, equation (3.72) is substituted, which results in

$$
d_{0} \approx \frac{2 R_{\mathrm{aper}} \lambda_{0}}{w \pi \mathrm{NA}}
$$

Substitution of equation (4.2) and the numerical values $\lambda_{0}=1064 \mathrm{~nm}$ and NA $=0.7$ in equation (4.4) yields $d_{0}=1.47 \mu \mathrm{m}$ This value is slightly smaller than the simulated spot size of $1.60 \mu \mathrm{m}$. This difference can be explained by the neglect of aperture diffraction effects in the analytical approximation.

For beam profile (3), the radial location $R$ of the first minimum on the Airy disk is given by [158]

$$
R=\frac{1.22 \lambda_{0}}{2 \mathrm{NA}} \approx 0.93 \mu \mathrm{m},
$$

which is close to the numerical value of $0.92 \mu \mathrm{m}$, which was computed on a grid with a radial spacing of $0.04 \mu \mathrm{m}$.

One of the approximations that were applied in the beam propagation model, is that the wave is paraxial (see section 3.3.2). To assess the error that is introduced by this approximation, the beam profile shown in figure 4.2 has been recomputed using the PSF Lab software [159]. This solver is based on an angular decomposition method for stratified media described in reference [160]. The obtained solutions satisfy Maxwell's equations. In the non-paraxial case, the coverslip correction needs to be considered (see section 5.4.1). Here, it was assumed that the coverslip correction is matched to the focus depth and the refractive index of silicon.

The simulated intensity profile without the paraxial approximation, computed with the PSF Lab software, is shown in figure 4.4. When comparing this profile with the computation which includes the paraxial approximation shown in figure 4.2 , it can be observed that the paraxial approximation provides an excellent match to the non-paraxial solution, despite the relatively high numerical aperture of 0.7 .

While the non-paraxial model only computes the propagation through a homogeneous medium, taking no absorption or changes in the refractive index into account, it requires at least three orders of magnitude more computation time compared with the paraxial beam propagation model presented in section $3 \cdot 3$.

The error caused by the paraxial solver, for the case of a homogeneous medium, is not fully representative for the conditions encountered during the laser pulse. Therefore, the actual error that is induced by the paraxial approximation may be larger than the comparison between figures 4.2 and 4.4 suggests. 


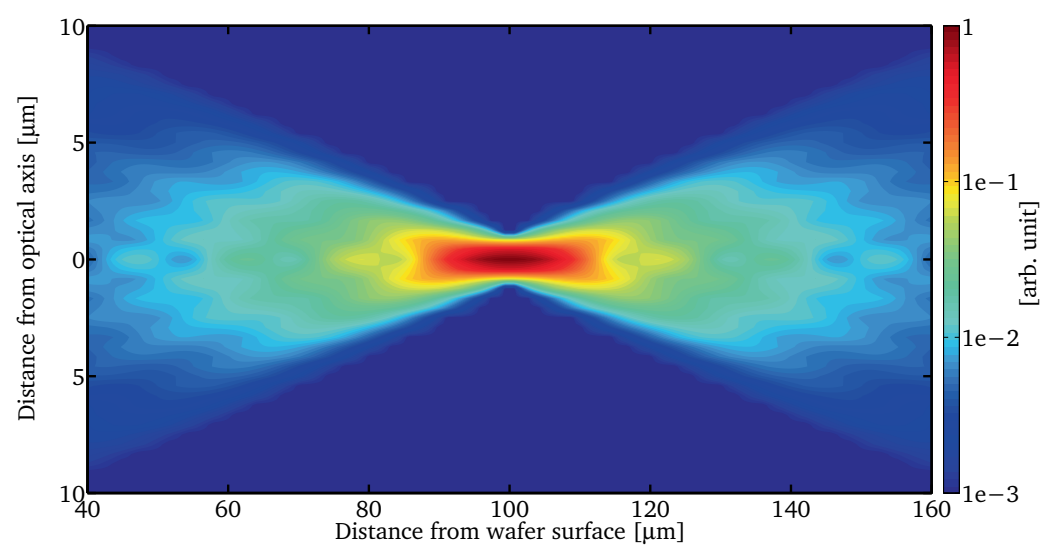

Figure 4.4: Cross-section of the laser intensity distribution [arbitrary unit] in the bulk of silicon, based on an electromagnetic wave that satisfies Maxwell's equations. No absorption was included. The laser beam at the back of the objective is Gaussian with the $1 / e^{2}$ beam width filling $80 \%$ of the aperture. Wavelength: $1064 \mathrm{~nm}$. The beam propagation direction is from left to right.

\subsection{Distributions of physical quantities}

In this section, simulated distributions of several physical quantities inside silicon will be presented and discussed. The quantities that are considered are the lattice and electron temperatures, the carrier density, the mechanical stress and the laser intensity.

\subsubsection{Lattice and electron temperatures}

The maximum lattice temperatures that were reached during the simulated time period of four times the FDHM pulse duration are shown in figures $4.5 \mathrm{a}$ and $4.5 \mathrm{~b}$. The laser parameters correspond to near-optimal processing conditions (see section 4.5.2). The black areas indicate the regions where the silicon has been molten. For a wavelength $\lambda_{0}$ of $1064 \mathrm{~nm}$ and a pulse duration $t_{p}$ of $5 \mathrm{~ns}$, the volume in which the temperature is significantly above the initial temperature is larger compared with $\lambda_{0}=1550 \mathrm{~nm}$ and $t_{p}=1 \mathrm{~ns}$, in spite of the smaller spot size. Consequently, the total molten volume is also smaller for $1064 \mathrm{~nm}$, as more energy is used for heating but not melting of material away from the focal plane.

The delocalization of the laser energy absorption at $1064 \mathrm{~nm}$ is caused by linear interband absorption. The $1550 \mathrm{~nm}$ wavelength requires two-photon absorption to initiate the absorption of laser energy, which is negligible at low intensities. Consequently, efficient absorption of laser energy only takes place in the proximity of the focal plane. At $1064 \mathrm{~nm}$, linear interband absorption occurs throughout the entire path of the laser beam inside silicon. Apart from energy losses, this results in the generation of photo-ionized carriers above the focus (see section 4.3.2), causing plasma defocussing of the laser beam (see section 4.3.4) and free carrier absorption. 


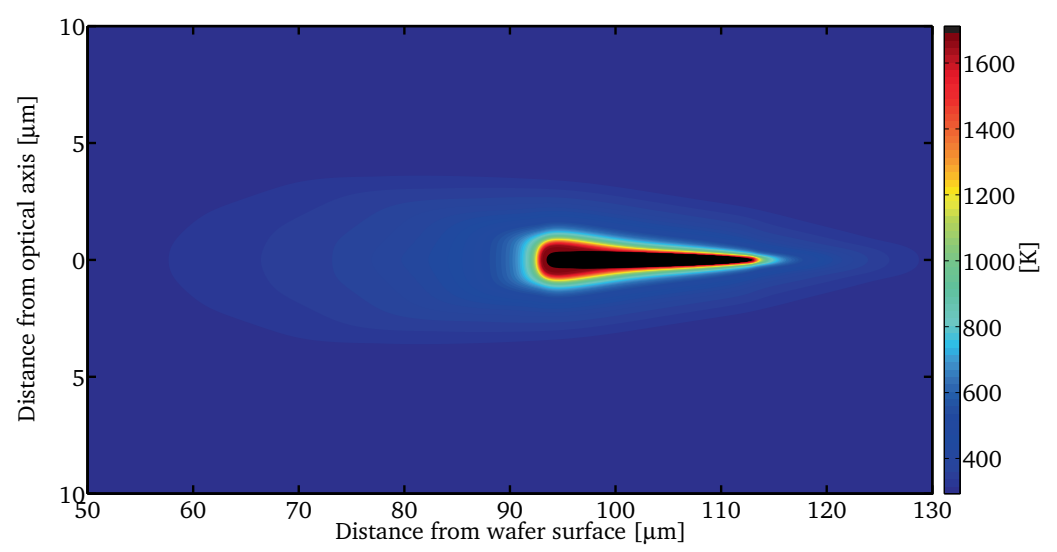

(a) Wavelength: $1064 \mathrm{~nm}$, pulse duration (FDHM): $5 \mathrm{~ns}$.

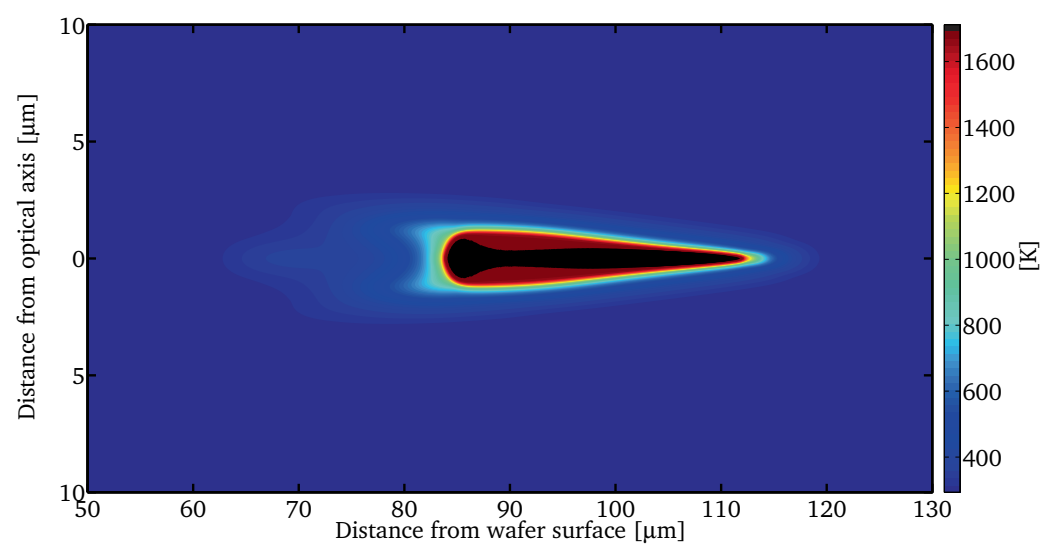

(b) Wavelength: $1550 \mathrm{~nm}$, pulse duration (FDHM): $1 \mathrm{~ns}$.

Figure 4.5: Cross-sections of the maximum lattice temperatures [K], during the entire simulated time period. Pulse energy: $1 \mu \mathrm{J}$. The beam propagation direction is from left to right. 
Figure 4.6 shows the time history of the lattice temperature, electron temperature and instantaneous laser power, for a pulse duration of $500 \mathrm{ps}$ and a wavelength of $1550 \mathrm{~nm}$. The temperatures are plotted at the centroid of the volume that reached the liquid phase during the laser pulse. A shorter pulse duration was selected compared with figures $4.5 \mathrm{a}$ and $4.5 \mathrm{~b}$ to demonstrate the effect of the electron-phonon coupling. The distributions were stored every $40 \mathrm{ps}$, the time steps used by the numerical model were significantly smaller. The electron temperature is plotted until the material has reached the liquid phase.

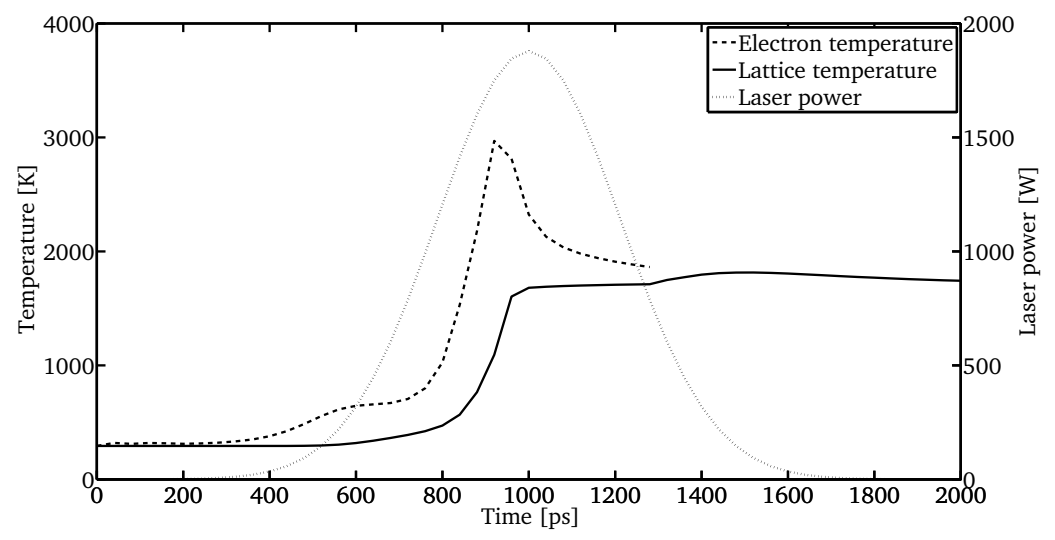

Figure 4.6: Lattice and electron temperatures and laser power as a function of time, at the centroid of the volume that reached the liquid phase during the pulse. Wavelength: $1550 \mathrm{~nm}$, pulse energy: $1 \mu \mathrm{J}$, pulse duration (FDHM): 500 ps.

At first, the lattice temperature shows a strong positive gradient until the temperature approaches the melting temperature. A slight positive gradient remains due to the apparent specific heat capacity method that was employed to take the latent heat into account (see section 3.2.2. After the material has reached the liquid phase, the further temperature increase is limited. This is caused by two phenomena. First, the laser power is decreasing at this point in time. Secondly, a high density of photo-ionized carriers has been formed above the location at which the temperatures are plotted. Therefore, the majority of the laser energy is absorbed before it reaches this location.

The largest imbalance between the lattice and electron temperatures in figure 4.6 is $1814 \mathrm{~K}$, which occurs just before the silicon reaches the liquid phase. At longer pulse durations, this temperature difference will be smaller. Shorter pulse durations than $500 \mathrm{ps}$ are not relevant, as they do not result in the formation of subsurface modifications (see section 4.5.2.

\subsubsection{Carrier density}

The maximum carrier densities that occurred during the time period until melting took place are shown in figures $4.7 \mathrm{a}$ and $4.7 \mathrm{~b}$. Material first reached the liquid phase at times of 123 ps and 46 ps before the peak power of the laser pulse, for the wavelengths of 1064 and $1550 \mathrm{~nm}$ respectively. The maximum carrier densities that were predicted by the 
simulations are $3 \cdot 10^{26} 1 / \mathrm{m}^{3}$ for $1064 \mathrm{~nm}$ and $3.5 \cdot 10^{26} 1 / \mathrm{m}^{3}$ for $1550 \mathrm{~nm}$. As is shown in section 4.5.2, the effect of carrier diffusion is not strong for short nanosecond pulses. Consequently, the carrier density is mainly determined by the absorption of photons and Auger recombination.

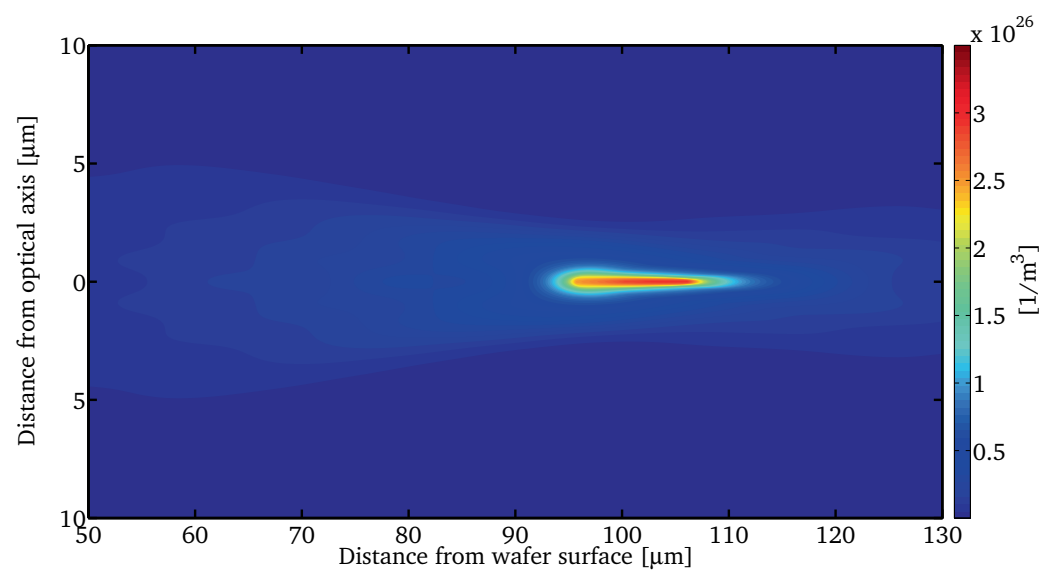

(a) Wavelength: $1064 \mathrm{~nm}$, pulse duration (FDHM): $5 \mathrm{~ns}$.

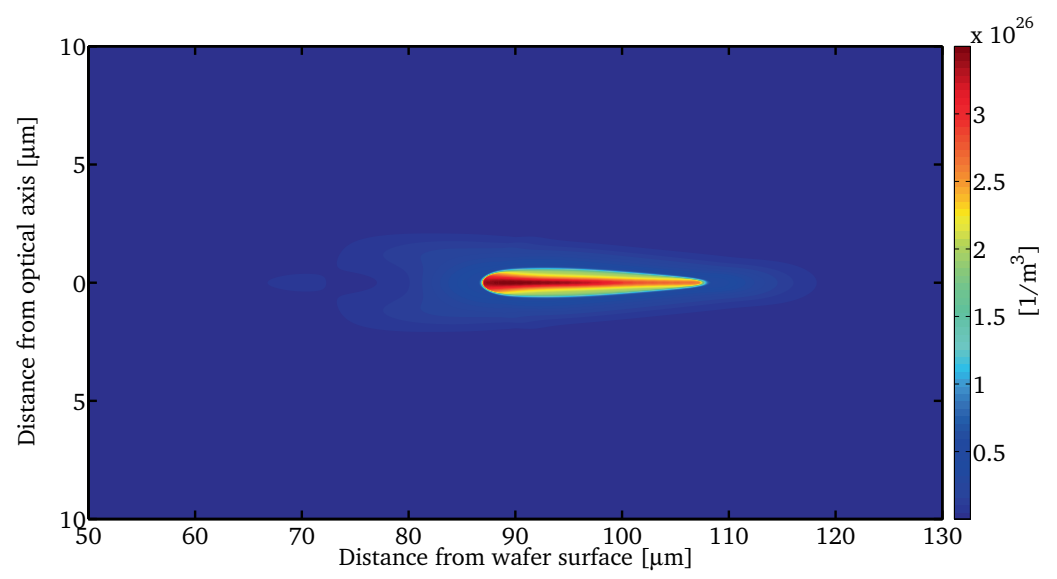

(b) Wavelength: $1550 \mathrm{~nm}$, pulse duration (FDHM): $1 \mathrm{~ns}$.

Figure 4.7: Cross-sections of the maximum carrier densities $\left[1 / \mathrm{m}^{3}\right]$ that occurred until the onset of melting. Pulse energy: $1 \mu \mathrm{J}$. The beam propagation direction is from left to right.

At distances of less than $60 \mu \mathrm{m}$ from the surface, no appreciable increase in carrier density is visible for the $1550 \mathrm{~nm}$ wavelength, while this does not hold for a wavelength of $1064 \mathrm{~nm}$. This improved confinement is due to the fact that the $1550 \mathrm{~nm}$ process relies solely on two-photon absorption to generate free carriers, for moderate lattice temperatures. Therefore, no effective absorption mechanism that moves electrons from the valence to the conduction band is present at low light intensities, i.e. at locations close to the surface. 


\subsubsection{Mechanical stress}

In this section, simulation results which include the modelling of stresses that are induced by thermal expansion are discussed. For a pulse energy of $1 \mu \mathrm{J}$, a pulse duration of $5 \mathrm{~ns}$ and a wavelength of $1064 \mathrm{~nm}$, the largest major principal stress, in the time period before the onset of melting, was equal to $1.4 \mathrm{GPa}$ (see figure 4.8). Here, largest is defined as having the greatest absolute value. For the same pulse energy, a pulse duration of $1 \mathrm{~ns}$ and a wavelength of $1550 \mathrm{~nm}$, a nearly identical value was found. In both cases, this value corresponds to a compressive stress. Note that the anisotropy of silicon cannot be taken into account in an axisymmetric coordinate system. Therefore, the values provided above are an approximation.

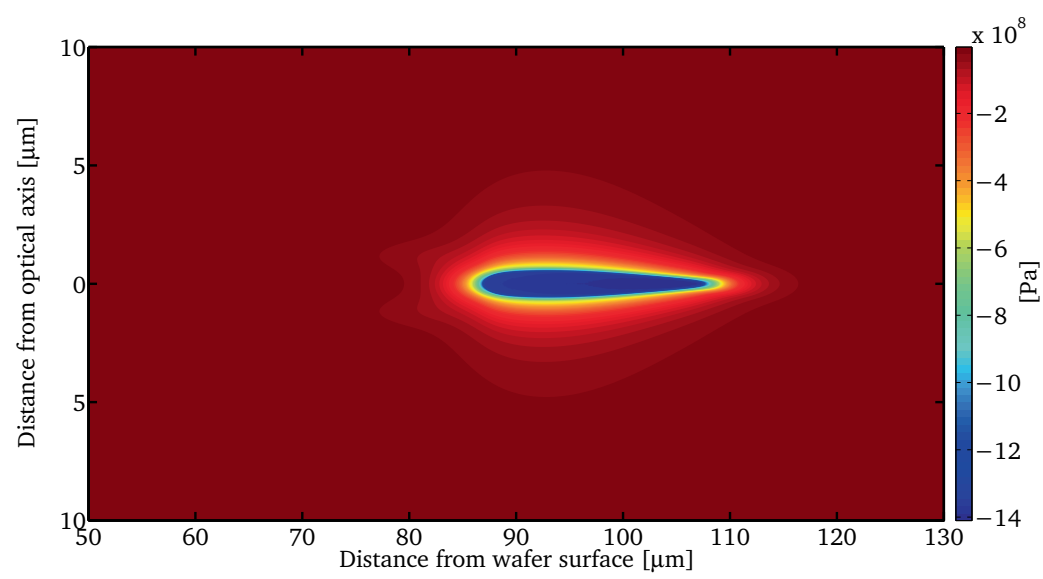

Figure 4.8: Cross-section of the largest major principal stresses [Pa] that occurred until the onset of melting. Negative values are compressive stresses while positive values are tensile stresses. Wavelength: $1064 \mathrm{~nm}$, pulse energy: $1 \mu \mathrm{J}$, pulse duration (FDHM): $5 \mathrm{~ns}$. The beam propagation direction is from left to right.

The major principal stress is the largest of the principal stresses. The sign indicates whether the stress is a tensile or a compressive stress. Only the magnitude of the stress is plotted in figure 4.8 , the direction of the stress is location-dependent. Since the azimuthal stress $\sigma_{\theta}$ is by definition a normal stress in an axisymmetric coordinate system, the principal stresses were computed by rotating orthogonal coordinates in the $r \%$ plane such that the shear stress is equal to zero. The normal stresses in this orientation together with $\sigma_{\theta}$ constitute the three principal stresses. The principal stresses in the $r z$ plane are given by

$$
\frac{\left(\sigma_{z}+\sigma_{r}\right)}{2} \pm \sqrt{\left(\frac{\sigma_{z}-\sigma_{r}}{2}\right)^{2}+\sigma_{r z}^{2}}
$$

\subsubsection{Laser intensity}

The laser intensity distributions inside a silicon wafer, at the time step just before melting took place, are shown in figures 4.9 and 4.10 . Note that different scales are used in these 
figures due to the difference in pulse duration. It can be observed that the beams retain an intensity profile that is close to the profile under equilibrium conditions, which is shown in figure 4.2 for a wavelength of $1064 \mathrm{~nm}$, until they reach the vicinity of the geometrical focus, where the temperatures and carrier densities are well above their initial values.

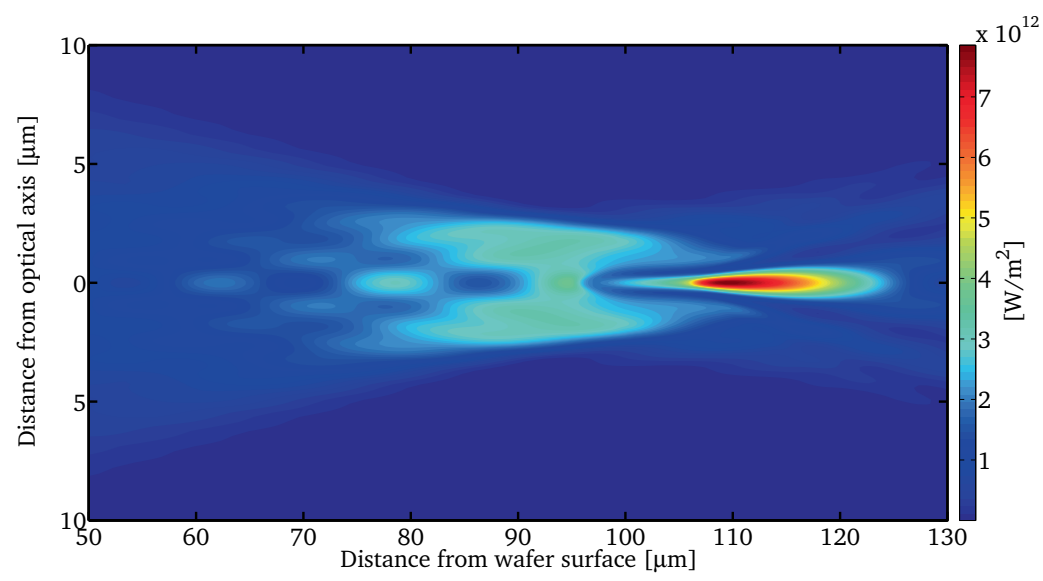

Figure 4.9: Cross-section of the laser intensity distribution $\left[\mathrm{w} / \mathrm{m}^{2}\right]$ just before subsurface melting. Wavelength: $1064 \mathrm{~nm}$, pulse energy: $1 \mu \mathrm{J}$, pulse duration (FDHM): $5 \mathrm{~ns}$. The beam propagation direction is from left to right.

The intensity distribution for the $1550 \mathrm{~nm}$ wavelength shows a sharp transition from a regime with little absorption to a regime with strong absorption of laser energy, including a near-zero intensity near the geometrical focus of the beam. This is due to the fact that two-photon absorption is exclusively responsible for the generation of electron-hole pairs at room temperature. Two-photon absorption scales with the square of the light intensity, resulting in preferential absorption of laser energy near the centre of the Gaussian beam.

The $1064 \mathrm{~nm}$ process is affected by plasma-induced beam defocussing, since this wavelength induces the generation of free carriers throughout the beam path inside silicon, irrespective of the laser intensity (see section 4.3.2). Plasma defocussing is caused by the dependence of the refractive index on the density of free carriers (see section 2.3). When disabling this dependence in the numerical simulations, it was found that the volume of molten material at $1064 \mathrm{~nm}$ is significantly enlarged and is comparable to the volume at $1550 \mathrm{~nm}$. At $1550 \mathrm{~nm}$, the influence of disabling this effect was found to be negligible.

The subsurface refractive index distribution corresponding to figure 4.9 is shown in figure 4.11. A refractive index drop towards the optical axis is visible before the beam reaches the vicinity of the focal plane, which is responsible for the defocussing of the laser beam. Close to the geometrical focus, the refractive index is above its equilibrium value of 3.57 , as a result of the high temperatures in this area. The refractive index changes that take place here are less relevant than those in the preceding beam path. Close to the focus, the laser energy is absorbed over a distance of a few micrometres. Because of this small distance, moderate focussing or defocussing of the beam due to index gradients does not have a significant impact on the subsurface laser intensity distribution. 


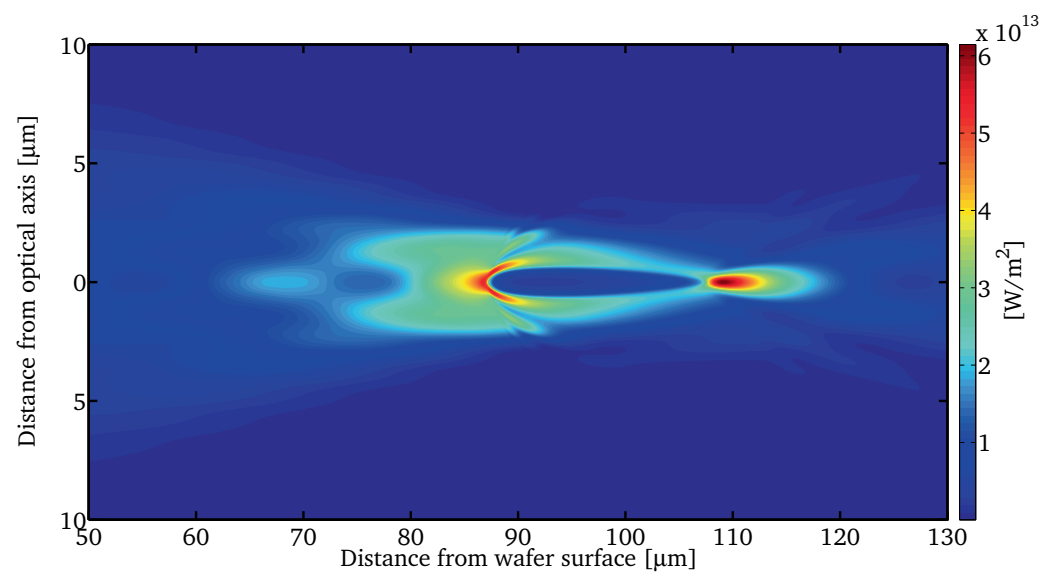

Figure 4.10: Cross-section of the laser intensity distribution $\left[\mathrm{w} / \mathrm{m}^{2}\right]$ just before subsurface melting. Wavelength: $1550 \mathrm{~nm}$, pulse energy: $1 \mu \mathrm{J}$, pulse duration (FDHM): $1 \mathrm{~ns}$. The beam propagation direction is from left to right.

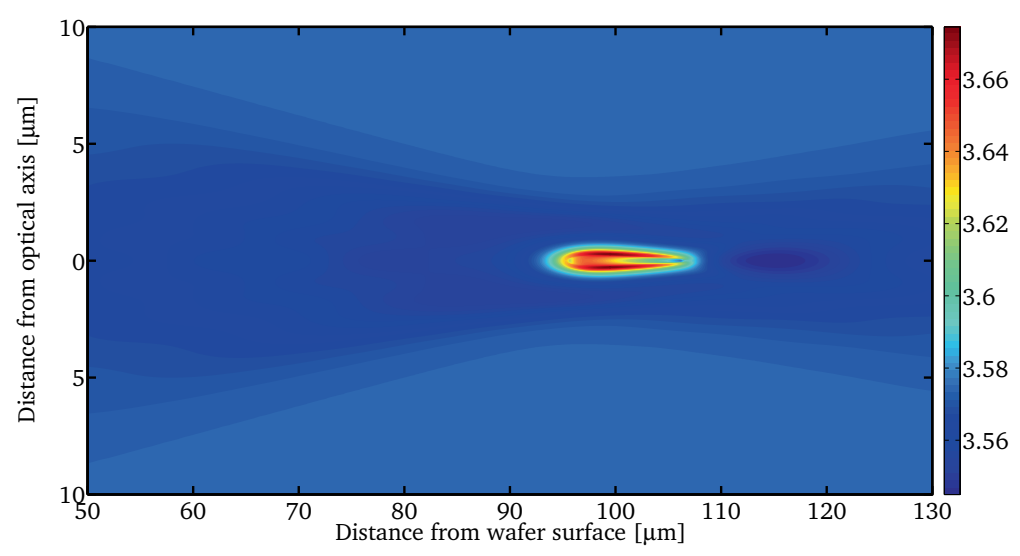

Figure 4.11: Cross-section of the refractive index just before subsurface melting. Wavelength: $1064 \mathrm{~nm}$, pulse energy: $1 \mu \mathrm{J}$, pulse duration (FDHM): $5 \mathrm{~ns}$. The beam propagation direction is from left to right. The refractive index under equilibrium conditions is 3.57 . 


\subsection{Modification mechanisms}

Three mechanisms that can cause the formation of a subsurface modification are included in the model (see section 3.2.6). These mechanisms are thermal melting, non-thermal melting and mechanical stresses. In this section, the simulation results are interpreted in terms of which mechanisms are responsible for the production of modifications.

\section{Non-thermal melting}

Non-thermal melting of silicon occurs when the carrier density exceeds the critical value $N_{\text {crit }}$, which is approximately $2.6 \cdot 10^{27} 1 / \mathrm{m}^{3}$ (see section 3.5 . . It was found that the density for non-thermal melting has not been exceeded during any of the simulations. The maximum carrier densities before the onset of melting, shown in section 4.3.2, are about a factor ten below the critical value. These conditions correspond to those that yield the largest modifications, as measured by their length along the optical axis.

The simulation result that non-thermal melting does not occur is plausible, as it was found that pulses in the short to long nanosecond range are required to selectively absorb laser energy in silicon, for the wavelengths and optical system that were considered (see section 4.5.2. For surface modifications, short picosecond pulses instead of nanosecond pulses are required to induce non-thermal melting of silicon [75]. For nanosecond pulses, the Auger recombination of electron-hole pairs is too strong to reach sufficiently high carrier densities for non-thermal melting.

\section{Mechanical stresses}

The largest major principal stress for near-optimal processing conditions, before the onset of subsurface melting, was found to be $1.4 \mathrm{GPa}$ (see section 4.3.3). This value is well below the ultimate tensile stress of silicon of $7 \mathrm{GPa}$ (see section 2.6). Moreover, as can be observed in figure 4.8 , the major principal stresses are almost exclusively compressive stresses, implying that even higher stresses are required to induce a permanent change to the material. The mechanical simulations include some uncertainty due to the use of an axisymmetric coordinate system. However, the differences between the simulated stresses and the ultimate tensile stress are sufficiently large to exclude the possibility of stress-induced modifications before the onset of melting.

\section{Thermal melting and resolidification}

The numerical simulations showed that thermal melting of silicon occurs (see section 4.3.1). Since non-thermal melting and stress-induced modifications were ruled out before the onset of melting, thermal melting and resolidification is predicted to be the primary modification mechanism for the laser conditions that were considered. Because the numerical model is only capable of accurately simulating the stresses while the material is in the solid phase, stresses may contribute as a secondary mechanism after melting.

Melting and resolidification results in a permanent modification, in case the resolidification speed is too high for the silicon to recrystallize into its original crystalline structure, without the formation of lattice defects (see section 3.2.6). It is possible that part of the melt resolidifies into almost defect-free diamond cubic silicon, which cannot be distinguished from the base material. Moreover, strong anisotropy can occur when molten silicon resolidifies into a crystalline phase. Both effects were not included in the numerical model. 
The exact extent of the laser-modified volume will be evaluated experimentally in chapter 7 based on transmission electron microscopy.

To achieve thermal melting of silicon, the material has to be heated from room temperature to the melting point and the latent heat of fusion has to be overcome. The corresponding energy density is given by

$$
E_{m}=\int_{T_{a m b}}^{T_{m}} \rho c_{p}\left(T_{l}\right) d T_{l}+L_{m} .
$$

Based on the material data in table 3.1 , this yields an energy density of $6.97 \cdot 10^{-3} \mu \mathrm{J} / \mu \mathrm{m}^{3}$. The required absorption of laser energy, to induce subsurface melting, may be larger than the energy density defined by equation (4.7), when laser energy moves away from the location where it was initially absorbed due to the effect of diffusion. Diffusion effects are especially of importance when employing laser pulses with a duration beyond $100 \mathrm{~ns}$ (see section 4.5.2. .

\subsection{Parameter studies}

In this section, parameter studies using the numerical model are presented, to assess the influence of variations in the processing conditions, material properties and initial conditions on the subsurface modification process. First, a characteristic dimension is selected to parametrise the shape of the modifications. Next, the influence of the pulse duration and energy, initial temperature, doping concentration and focus depth on this dimension will be evaluated, for both the 1064 and $1550 \mathrm{~nm}$ wavelengths.

\subsubsection{Parametrisation of the geometry of modifications}

To analyse the geometry of the modifications induced by different processing conditions, a characteristic parameter has to be selected that is suitable for comparison with experimental results. Based on the consideration of various modification mechanisms (see section 4.4), thermal melting and resolidification was found to be the primary modification mechanism. Therefore, the shape of the modifications was assumed to coincide with the volume of material that has been molten during the laser pulse.

Changes to the laser parameters were mainly found to affect the length of the volume of molten material along the optical axis. Therefore, the modification length (see figure 4.12) was selected to characterize the geometry of the modifications. Additionally, the modification length can be measured with sufficient accuracy during experimental parameter studies, making it a suitable choice for the comparison of experimental and numerical results (see section 6.4).

For pulse energies well above the modification threshold, discontinuous modifications may be found due to periodic fluctuations in the laser intensity along the optical axis, as a result of aperture diffraction. In these cases, the modification length was defined as the total length along the optical axis, from the first to the last modified material. 


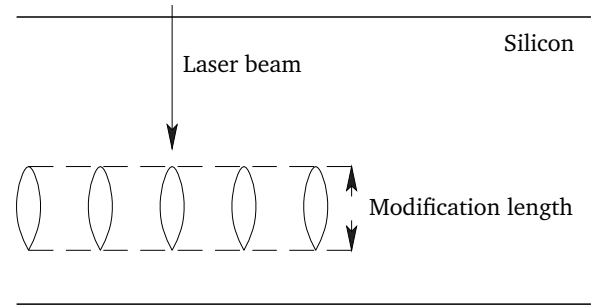

Figure 4.12: Definition of the length of subsurface modifications along the optical axis, inside the silicon wafer. The beam propagation direction is from top to bottom.

In the following parameter studies, the modification length along the optical axis will be plotted as a function of the parameter that is under investigation. The piecewise linear curves in these plots connect the data points. They do not resemble the actual distributions between these points.

\subsubsection{Pulse duration and energy}

For both the 1064 and $1550 \mathrm{~nm}$ wavelengths, the lengths of the subsurface modifications along the optical axis were determined from the simulation results, as a function of the pulse duration and pulse energy (see figures $4.13 \mathrm{a}$ and $4.13 \mathrm{~b}$ ).

According to the simulation results, both wavelengths can be applied for the production of subsurface modifications in silicon. For the pulse energies that were simulated $(0.5,1$ and $2 \mu \mathrm{J}$ ), the suitable pulse durations range from 500 ps to $250 \mathrm{~ns}$ for the $1064 \mathrm{~nm}$ process and from $500 \mathrm{ps}$ to $50 \mathrm{~ns}$ for a wavelength of $1550 \mathrm{~nm}$. The optimum pulse durations are around 5 ns and $1 \mathrm{~ns}$ for 1064 and $1550 \mathrm{~nm}$ respectively. These conditions result in the longest modifications for a fixed pulse energy.

Advantage of the $1064 \mathrm{~nm}$ process is that it is less sensitive to the choice of the pulse duration, as both single- and two-photon absorption may be employed, depending on the pulse duration. However, the $1550 \mathrm{~nm}$ wavelength is more efficient, as more material is modified while applying the same pulse energy, if the optimum processing conditions are selected. An important cause for the lower efficiency of the $1064 \mathrm{~nm}$ wavelength is the delocalization of the laser energy absorption due to plasma defocussing (see section 4.3.4). In the next sections, a more detailed comparison of both wavelengths will be performed, while varying the initial temperature, doping concentration and focus depth.

Boundaries of the process window To investigate the physical phenomena that govern the boundaries of the process window in terms of the pulse duration and energy, simulations have been run for a wavelength of $1064 \mathrm{~nm}$, while artificially enhancing Auger recombination and disabling two-photon absorption, or while disabling the effect of diffusion (see figure 4.14). The Auger recombination was enhanced by multiplying the Auger recombination coefficient by a factor hundred. Two-photon absorption was disabled by setting the two-photon absorption coefficient to zero. Finally, diffusion effects were 


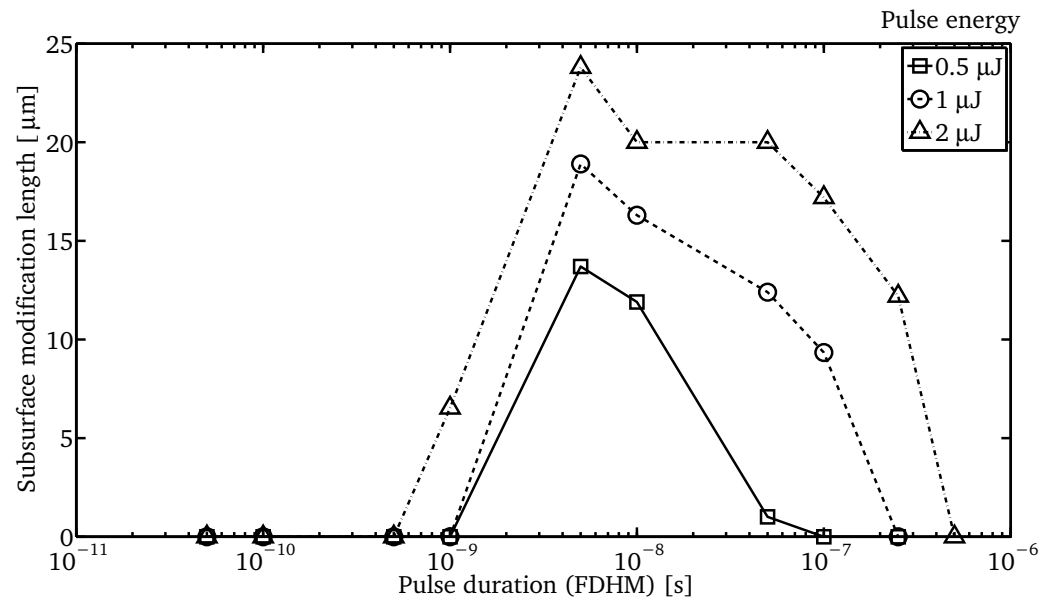

(a) Wavelength: $1064 \mathrm{~nm}$.

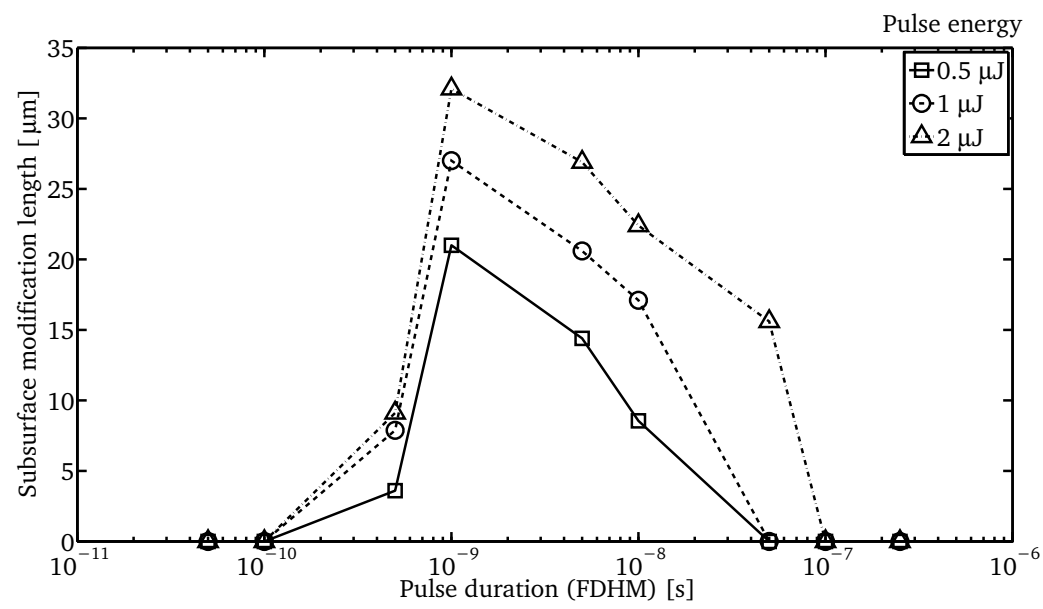

(b) Wavelength: $1550 \mathrm{~nm}$.

Figure 4.13: Subsurface modification length as a function of the pulse duration for three pulse energies. 


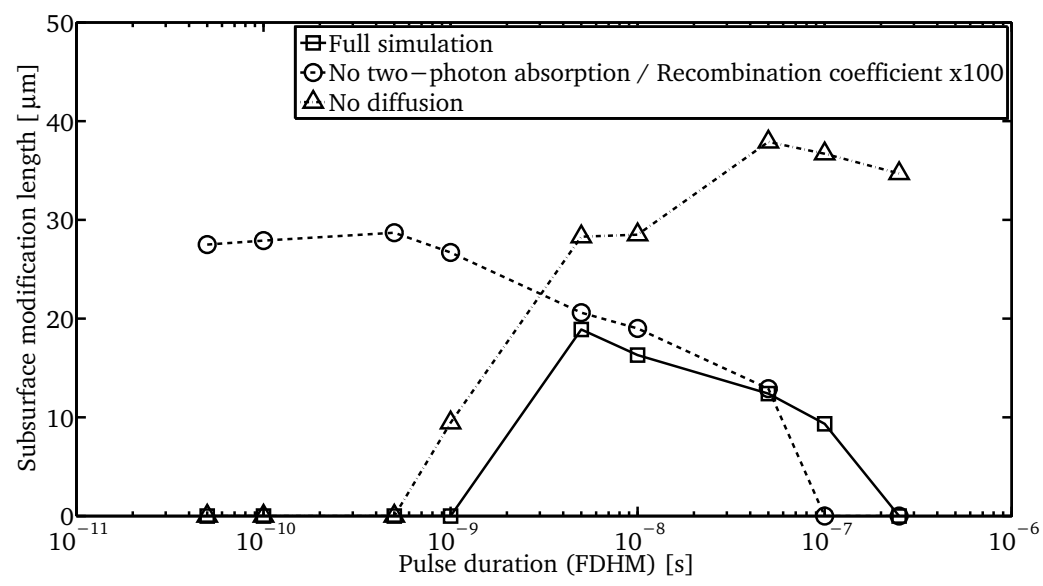

Figure 4.14: Subsurface modification length as a function of the pulse duration, while enhancing or disabling several physical phenomena. Wavelength: $1064 \mathrm{~nm}$, pulse energy: $1 \mu \mathrm{J}$.

disabled by zeroing the diffusion coefficients corresponding to the lattice heat conduction, electronic heat conduction and ambipolar diffusion of electron-hole pairs.

Upper boundary of the pulse duration For the $1064 \mathrm{~nm}$ wavelength, the upper limit to the pulse duration is related to the diffusion of heat and carriers away from the focus, which limits the ability to selectively generate high temperatures near or in the focus. When disabling this effect, long pulses of hundreds of nanoseconds hardly show any decrease in performance compared with shorter pulse durations (see figure 4.14). For the $1550 \mathrm{~nm}$ process, this effect is less relevant as the process already fails at shorter pulse durations, due to the lack of two-photon absorption (see figure 4.13b). The upper boundaries shown in figures $4.13 \mathrm{a}$ and $4.13 \mathrm{~b}$ are not absolute limits. When further increasing the pulse energy, modifications can also be created using longer pulses.

Lower boundary of the pulse duration The lower limit to the pulse duration is due to delocalization of the laser energy absorption. The laser pulse has to contain sufficient energy to melt a volume of material below the surface, as given by equation (4.7). If the laser pulse is too short, while keeping the pulse energy at the required level, the laser intensity well above the focus exceeds the threshold for effective two-photon absorption. Consequently, laser energy is absorbed at undesired locations and a too dense electron-hole plasma is formed above the focus of the beam. A contributing factor to the formation of this plasma is that less time is available for the recombination of electron-hole pairs, when reducing the pulse duration. The electron-hole plasma above the focal plane results in (1) energy losses due to free carrier absorption of laser energy and (2) plasma-induced defocussing of the beam. If a too short pulse duration is selected, eventually a surface instead of a subsurface modification will be created when increasing the pulse energy. When artificially disabling two-photon absorption and increasing the Auger recombination 
coefficient in the simulations, ultra-short pulses in the picosecond range also result in the formation of subsurface modifications (see figure 4.14).

The refractive index profile at the peak power of a 50 ps pulse with a wavelength of $1064 \mathrm{~nm}$ is shown in figure 4.15. The refractive index drops towards the optical axis due to its dependence on the density of free carriers, causing the laser beam to defocus. Contrary to the situation for a nanosecond pulse (see figure 4.11), the refractive index is below its equilibrium value of 3.57 throughout the entire computational domain.

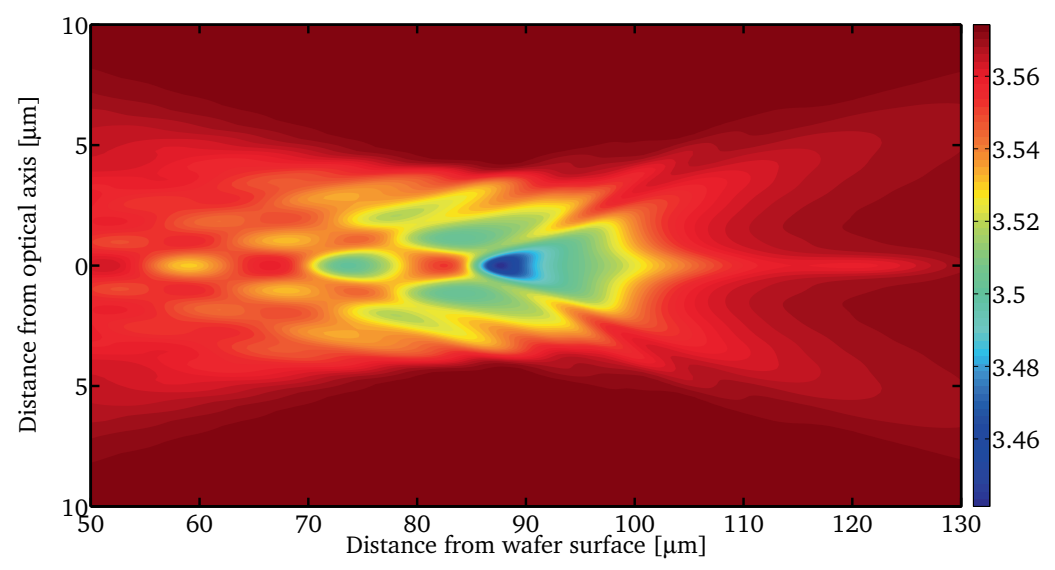

Figure 4.15: Cross-section of the refractive index at the peak power of the laser pulse. Wavelength: $1064 \mathrm{~nm}$, pulse energy: $1 \mu \mathrm{J}$, pulse duration (FDHM): 50 ps. The beam propagation direction is from left to right. The refractive index under equilibrium conditions is 3.57 .

For the pulse energies that were employed during the simulations shown in figures $4.13 \mathrm{a}$ and $4.13 \mathrm{~b}$, a positive relation between the pulse energy and modification length exists. This trend is not consistent when further increasing the pulse energy. If the pulse duration is fixed, higher pulse energies result in higher instantaneous light intensities, enabling two-photon absorption at larger distances from the focal plane. Therefore, the lower boundary of the pulse duration will shift to longer pulse durations.

Influence of the Kerr effect For a pulse energy of $1 \mu \mathrm{J}$, the lower boundary of the pulse duration at $1064 \mathrm{~nm}$ was found to be around $1 \mathrm{~ns}$ (FDHM). This corresponds to a peak power of $0.94 \mathrm{~kW}$, for a laser pulse with a Gaussian temporal profile. At wavelength of $1064 \mathrm{~nm}$, the approximate critical power for self-focussing according to equation (2.1) is $17 \mathrm{~kW}$. Consequently, the lower limit to the pulse duration is not governed by the Kerr effect for the conditions under consideration.

Due to the Kerr effect, the hypothetical case in which two-photon absorption is disabled and the Auger recombination is enhanced, as shown in figure 4.14, will not result in the formation of subsurface modifications, when decreasing the pulse duration below the range that is plotted. Such hypothetical conditions cannot be modelled by solving a nonlinear Schrödinger equation in an axisymmetric coordinate system, as it becomes mathematically 
singular [122, 161]. This singularity does not occur when considering the Kerr effect and two-photon absorption together [122].

\subsubsection{Initial temperature}

The length of the subsurface modifications along the optical axis, as a function of the wavelength and initial lattice temperature, is shown in figure 4.16. In practise, such an increase in the initial temperature may be caused by heat input from neighbouring pulses or by the accumulation of heat over long processing times. This is especially expected to be an issue when machining at high laser repetition frequencies.

While a constant initial temperature can represent overall heating of the wafer, nonaxisymmetric temperature profiles will occur when producing line patterns of subsurface modifications. Such profiles cannot be simulated in an axisymmetric coordinate system as used by the current numerical model. Non-homogeneous initial temperatures will have a stronger detrimental effect on the performance of the process than homogeneous heating, due to the corresponding refractive index gradients which affect the intensity profile of the laser beam.

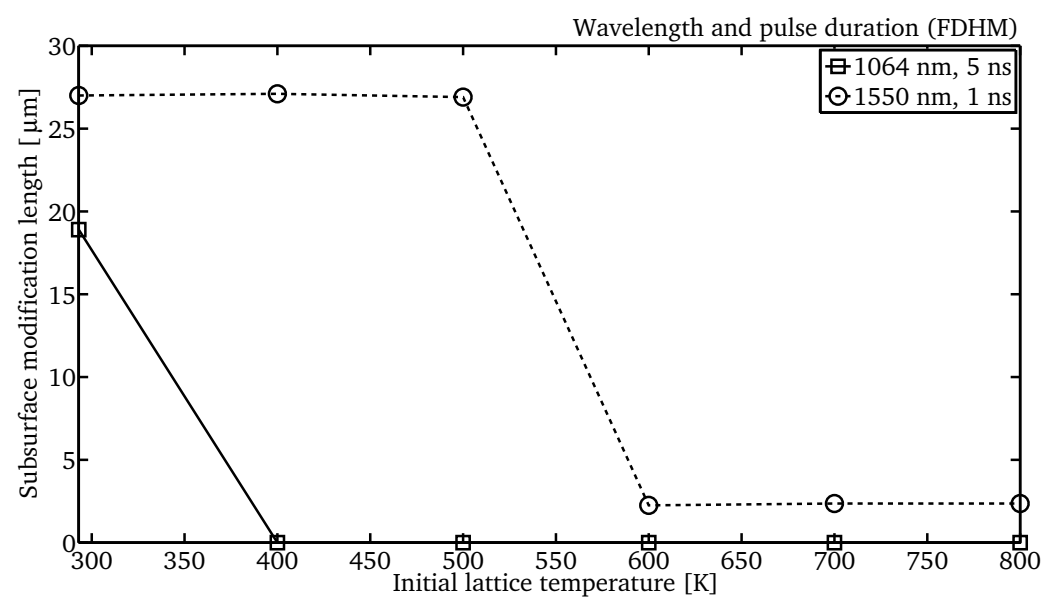

Figure 4.16: Subsurface modification length as a function of the initial lattice temperature for two wavelengths. Pulse energy: $1 \mu \mathrm{J}$.

When comparing the 1064 and $1550 \mathrm{~nm}$ wavelengths, a large difference in performance at elevated initial lattice temperatures can be observed. An increase in temperature of $100 \mathrm{~K}$ greatly enhances the linear interband absorption for the $1064 \mathrm{~nm}$ wavelength, by shrinking the band gap and increasing the probability of phonon absorption (see section 2.3). Consequently, sufficient laser energy does not reach the vicinity of the focal spot, causing the process to fail. The $1550 \mathrm{~nm}$ process is unaffected by a temperature increase of about $200 \mathrm{~K}$. Only at temperatures around $600 \mathrm{~K}$, it no longer performs satisfactorily. This is due to the increase in intrinsic carrier density and free carrier cross-section, resulting in stronger free carrier absorption. 


\subsubsection{Doping concentration}

In addition to the initial temperature, the concentration of dopants influences the formation of laser-induced subsurface modifications. While the band gap of silicon shrinks with an increasing dopant concentration due to band gap narrowing, the optical band gap remains relatively constant, as the band gap narrowing is counteracted by band filling [162]. Since the optical band gap determines the laser energy absorption, only the effect of ionized impurities on the free carrier absorption was considered. As the numerical model assumes an equal number of electrons and holes, it does not distinguish between $\mathrm{p}$ - and n-doping when computing the material properties. It was assumed that the contributions of holes and electrons to the free carrier absorption are identical.

The length of the subsurface modifications, as a function of the wavelength and the concentration of ionized impurities, is shown in figure 4.17. A focus depth of $500 \mu \mathrm{m}$ was selected for these simulations, as the effect of ionized impurities is especially of importance when processing relatively thick wafers. The processing strategies based on the 1064 and $1550 \mathrm{~nm}$ wavelengths both show a strong decrease in performance, when the concentration of ionized impurities is increased beyond $10^{18} 1 / \mathrm{cm}^{3}$. The effect on the $1550 \mathrm{~nm}$ process is somewhat stronger, due to the larger free carrier cross-section at this wavelength (see section 3.5). The concentrations of ionized impurities at which the process fails, which are shown in figure 4.17, are not absolute limits. It is possible to compensate for the effect of free carrier absorption to some extent by increasing the pulse energy.

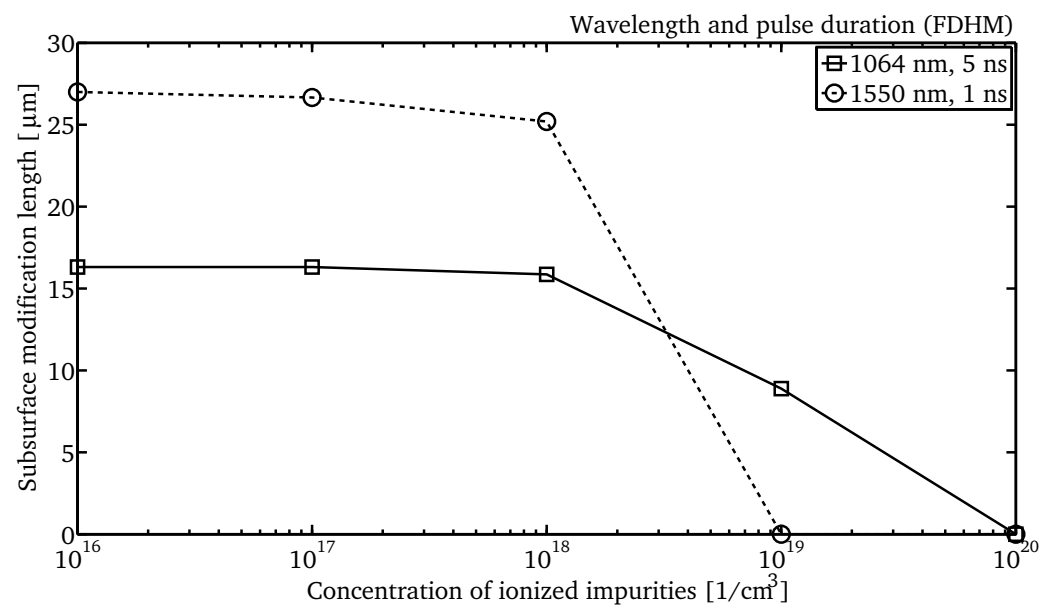

Figure 4.17: Subsurface modification length as a function of the concentration of ionized impurities for two wavelengths. Pulse energy: $1 \mu \mathrm{J}$, focus depth: $500 \mu \mathrm{m}$.

\subsubsection{Focus depth}

Finally, the influence of the focus depth on the length of the subsurface modifications has been investigated (see figure 4.18). The focus depth is defined as the distance between the wafer surface and the geometrical focus of the laser beam. For the case of intrinsic silicon, the $1550 \mathrm{~nm}$ process was found to be unaffected by any change in focus depth in the range 


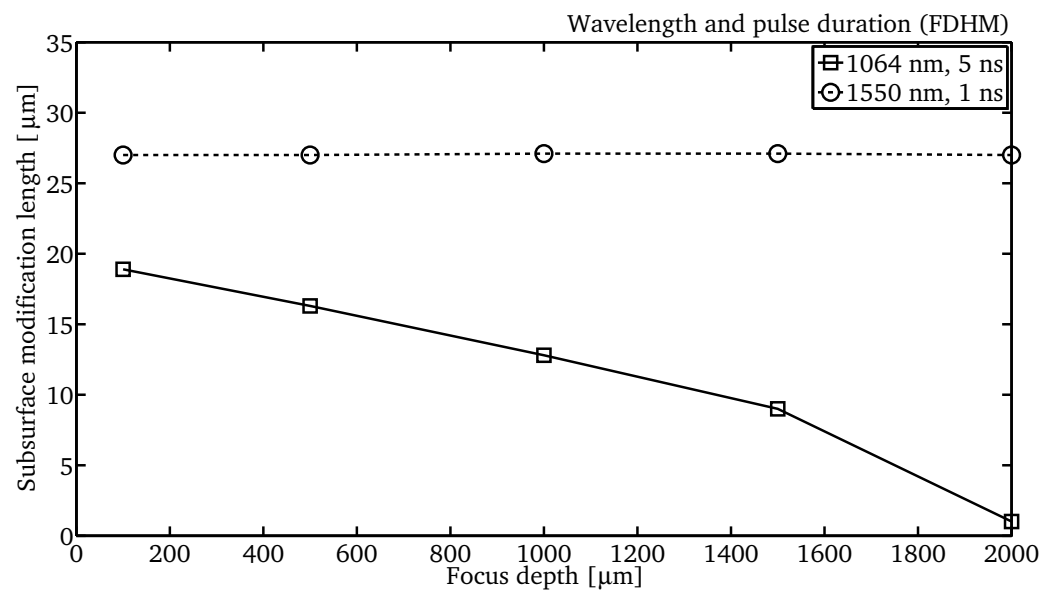

Figure 4.18: Subsurface modification length as a function of the focus depth for two wavelengths. Pulse energy: $1 \mu \mathrm{J}$.

that was simulated (up to $2 \mathrm{~mm}$ ). At $1064 \mathrm{~nm}$, the modification length decreases for larger focus depths.

The $1550 \mathrm{~nm}$ process is robust when varying the focus depth, because the laser energy absorption at low light intensities is limited to free carrier absorption by the intrinsic concentration of carriers. Interband absorption does not occur at room temperature, as the photon energy is well below the band gap. At $1064 \mathrm{~nm}$, losses due to linear interband absorption occur throughout the beam path inside silicon. Moreover, the free carriers that are generated in this manner further enhance the absorptivity.

As long as the doping concentration is not too high, the $1550 \mathrm{~nm}$ process allows for the production of laser-induced modifications deep inside silicon, without significant energy losses compared with the fabrication of near-surface modifications.

\subsection{Summary}

Numerical simulations of the subsurface laser-material interaction in silicon have been performed, while varying the wavelength, pulse duration, pulse energy, beam shape, initial temperature, focus depth and doping concentration. Results of simulations that were carried out for wavelengths of 1064 and $1550 \mathrm{~nm}$ were compared. Both wavelengths were found to be suitable for the formation of subsurface modifications in silicon, if appropriate processing conditions are selected.

The simulations predicted that non-thermal melting does not occur. Moreover, stresses exceeding the ultimate tensile strength were not found, while the material was in the solid phase. Since thermal melting was predicted, subsurface melting of silicon followed by fast resolidification is expected to be the primary modification mechanism.

For the $1064 \mathrm{~nm}$ wavelength, the simulations showed that pulse durations up to a few hundred nanoseconds can be employed to induce modifications, based on single-photon 
absorption of laser energy. Longer pulses are ineffective to form modifications, due to the diffusion of heat and free carriers. For the $1550 \mathrm{~nm}$ wavelength, two-photon absorption is required to initiate the formation of subsurface modifications. Since high light intensities are required to trigger two-photon absorption, it was found that the upper limit to the pulse duration is in the order of tens of nanoseconds. The pulse energies that were needed, for both wavelengths, are in the order of a microjoule.

For both wavelengths, a lower limit to the pulse duration, to form modifications, was also found to exist. The pulse energy is determined by the required energy to melt a volume of silicon. When combining this energy level with a too short pulse duration, the instantaneous power of the laser beam becomes too high to limit the multiphoton absorption of laser energy to the vicinity of the focus. Consequently, apart from direct energy losses due to multiphoton absorption, a dense electron-hole plasma is formed above the focus. This plasma results in free carrier absorption of laser energy and plasma-induced beam defocussing. For the conditions under consideration, these phenomena limit the usability of short pulses before the Kerr effect becomes relevant.

A comparison was performed regarding the performance of the processing strategies based on wavelengths of 1064 and $1550 \mathrm{~nm}$. The $1550 \mathrm{~nm}$ process was found to result in longer modifications for the same pulse energy. At $1064 \mathrm{~nm}$, free carriers are generated throughout the path of the laser beam inside silicon, due to linear interband absorption, resulting in energy losses and plasma defocussing. Moreover, the $1064 \mathrm{~nm}$ process is precisely matched to the band gap of silicon, making it sensitive to band gap narrowing caused by an increase in the initial temperature. Since energy losses at low laser intensities are negligible at $1550 \mathrm{~nm}$, this wavelength is well suited for the production of subsurface modifications at a depth of several millimetres below the surface.

In order to assess the accuracy of the numerical simulations, simulation results will be compared with experimental data in chapter 6 . 


\section{Experimental set-up and analysis tools}

In this chapter, the experimental set-up that was designed to tightly focus laser pulses inside silicon is discussed. In addition to the laser sources, optics and mechanical components of the set-up, the sample analysis tools will be presented.

\subsection{Introduction}

In chapter 4 simulation results were presented that predict the feasibility of producing subsurface modifications in silicon under various processing conditions. To validate the numerical results using experimental data, an experimental set-up has been constructed to tightly focus laser pulses inside silicon. In this chapter, the design of the set-up will be discussed. Special attention is given to the focussing objective, as this is one of the most important components for the success of the subsurface modification process.

Besides the laser processing set-up, methods to analyse the presence of subsurface modifications, their geometry and their crystal structure will be discussed. These methods include non-destructive methods, as well as destructive methods that require additional machining of the samples after laser processing. Moreover, sample geometries are proposed that are adapted to the requirements of the analysis methods.

The processing conditions that can be generated by the set-up are not limited to those that were predicted to be suitable for the formation of subsurface modifications in silicon. This enables the validation of both positive and negative results of the numerical simulations.

\subsection{Laser sources}

Four laser sources were employed for the experiments on the formation of subsurface modifications in silicon. Their properties are listed in table 5.1. The wavelengths of the lasers are a close match to the wavelengths that were used during the numerical simulations (see section 4.1). The energy of the pulses emitted by these sources is a least $20 \mu \mathrm{J}$.

Two of the lasers sources that were employed are fibre lasers. Fibre lasers are well suited for the subsurface modification process, as they have an excellent beam quality and can produce medium energy pulses of tens to hundreds of microjoules. For the formation of subsurface modifications, low pulse energies of a few microjoules are sufficient, as the material only has to be locally modified instead of being removed. A high beam quality is beneficial to obtain a small focal spot inside the material.

Both fibre lasers employ a master oscillator power amplifier (MOPA) architecture. The $1549 \mathrm{~nm}$ fibre laser provides a fixed pulse duration of $3.5 \mathrm{~ns}$ (FDHM). At this wavelength, the laser process relies on two-photon absorption to generate an initial electron-hole plasma (see section 2.3). The $1061 \mathrm{~nm}$ fibre laser includes the option of changing the temporal 
Table 5.1: Properties of the laser sources that were employed to produce subsurface modifications in silicon. The pulse duration is defined as the full duration at half maximum (FDHM).

\begin{tabular}{llrl}
\hline $\begin{array}{l}\text { Manufacturer and } \\
\text { type }\end{array}$ & $\begin{array}{l}\text { Lasing medium and } \\
\text { pump source }\end{array}$ & $\begin{array}{r}\text { Wavelength and } \\
\text { pulse duration }\end{array}$ & $\begin{array}{l}\text { Beam quality and } \\
\text { polarization }\end{array}$ \\
\hline $\begin{array}{l}\text { Trumpf } \\
\text { Trumicro 5050 }\end{array}$ & Diode pumped & $\begin{array}{r}1030 \mathrm{~nm} \\
6.6 \mathrm{ps}\end{array}$ & $\begin{array}{l}M^{2}<1.3 \\
\text { Linear }\end{array}$ \\
\hline Spectra Physics & Nd:YAG rod & $1064 \mathrm{~nm}$ & Undefined \\
Quanta Ray GCR-270 & Flashlamp pumped & $8 \mathrm{~ns}$ & Linear \\
\hline SPI & Yb-doped silica fibre & $1061 \mathrm{~nm}$ & $M^{2}<1.3$ \\
SP-020P-A-EP-S-A-Y & Diode pumped & $2-460 \mathrm{~ns}$ & Random \\
\hline MWTechnologies & Er-doped silica fibre & $1549 \mathrm{~nm}$ & $M^{2}<1.1$ \\
MOPA-LF-1550 & Diode pumped & $3.5 \mathrm{~ns}$ & Random \\
\hline
\end{tabular}

shape of the seed pulses, to obtain amplified pulses with different pulse durations. The ability to generate longer nanosecond pulses at this wavelength allows for the investigation of a modification process that is solely based on single-photon absorption.

The manufacturers of the laser sources listed in table 5.1 use different definitions to quantify the power and energy stability of the laser output. For the experiments that will be presented in chapters 6 and 7 , the stability of the pulse energy is the most important. For the Spectra Physics source, $99 \%$ of the pulse energies are within $\pm 2 \%$ of the average value. For the SPI source, the root mean square of the energy deviations is smaller than $3 \%$. The мwтеchnologies source has a short term peak power stability of $\pm 5 \%$. For the Trumpf source, only the long term stability of the average power is specified as $\pm 1.7 \%$.

Pumping scheme of the fibre lasers Low pulse repetition frequencies in the order of $100 \mathrm{~Hz}$ were required to obtain a sufficiently large spacing between adjacent modifications (see section 5.5). The Spectra Physics laser source has a repetition rate of $10 \mathrm{~Hz}$. The Trumpf laser source includes a pulse picker to enable operation at low repetition frequencies. However, fibre lasers are typically designed to operate at far higher pulse repetition rates.

The MOPA architecture of the fibre lasers contains a seed laser that provides short pulses with a low pulse energy. In one or more amplification stages, the seed pulses are amplified to obtain the required pulse energy. While the amplifiers are intended to amplify seed pulses by means of stimulated emission, a certain amount of energy loss due to spontaneous emission will also occur. As part of the photons emitted by spontaneous emission are also guided by the fibre and are subsequently amplified, this will result in amplified spontaneous emission (ASE).

When intermittently triggering single pulses from a fibre laser or running the laser at low repetition frequencies, while continuously pumping the amplifiers, the contribution 
of ASE to the total average power of the laser will be large. This is due to the fact that the amplifiers are almost continuously in a state of maximum gain and energy storage, resulting in strong ASE, while the output power corresponding to the laser pulses is limited. Too strong ASE can result in the sample being damaged at undesired locations. Moreover, it complicates the measurements of the pulse energy, as one has to distinguish between the contribution of the actual laser pulses and the ASE. Typical repetition rates for which the average power due to ASE exceeds the power corresponding to the short pulses are in the order of several kilohertz [163].

To address the issue of ASE, the lasers were configured for a pulsed pumping scheme [164]. This means that the diodes are briefly switched on for a period of tens to hundreds of microseconds before a seed pulsed is released. After the laser pulse has been released, the pumping diodes are switched off. This pumping scheme results in minimal energy storage in the amplifiers between the laser pulses. The pulse energy can be controlled by adapting either the pumping time or the diode current.

Temporal pulse shapes To compare experimental results with numerical simulations, the temporal shapes of the laser pulses have to be known. The temporal profiles of the pulses emitted by the Spectra Physics, Trumpf and MwTechnolgies laser sources are best approximated by a Gaussian profile. The SPI laser source has a tunable pulse duration. For this source, the pulse shape depends on the pulse duration.

The temporal pulse shapes corresponding to the different pulse durations of the SPI source have been measured by a Thorlabs SV2-FC biased photodiode and a Lecroy WavePro 7200A oscilloscope. Both the diode and the oscilloscope have a bandwidth of $2 \mathrm{GHz}$. The pulse shapes were digitized using a sample frequency of $20 \mathrm{GS} / \mathrm{s}$ and were averaged over 1024 pulses.

The shapes of 8,240 and 460 ns (FDHM) pulses, which will be used for quantitative comparisons between experimental and modelling results (see section 6.6), are shown in figures 5.1, 5.2 and 5.3 respectively. Based on the measurement results, the $8 \mathrm{~ns}$ pulses of the SPI laser source will be approximated by a Gaussian temporal profile during numerical simulations, while the longer pulses will be modelled by assuming a constant power during the pulse (see section 3.3.1).

\subsection{Laser processing set-up}

In this section, the experimental set-up to focus laser pulses inside crystalline silicon is presented. Based on the wavelengths of the laser sources listed in section 5.2. the set-up was designed for wavelengths around 1064 and $1550 \mathrm{~nm}$. The microscope objective, including the influence of the coverslip correction, will be discussed in section 5.4.

A drawing of the experimental set-up is shown in figure 5.4. A photograph of the set-up connected to the $1549 \mathrm{~nm}$ laser source is presented in figure 5.5 . The manufacturers and part numbers of the components that are shown in these figures are listed in table 5.2 When different components were required for the 1064 and $1550 \mathrm{~nm}$ wavelengths, two part numbers are listed. The Trumpf and Spectra Physics lasers directly provide a linearly polarized free space beam. The optical fibres coming from the 1061 and $1549 \mathrm{~nm}$ fibre laser 


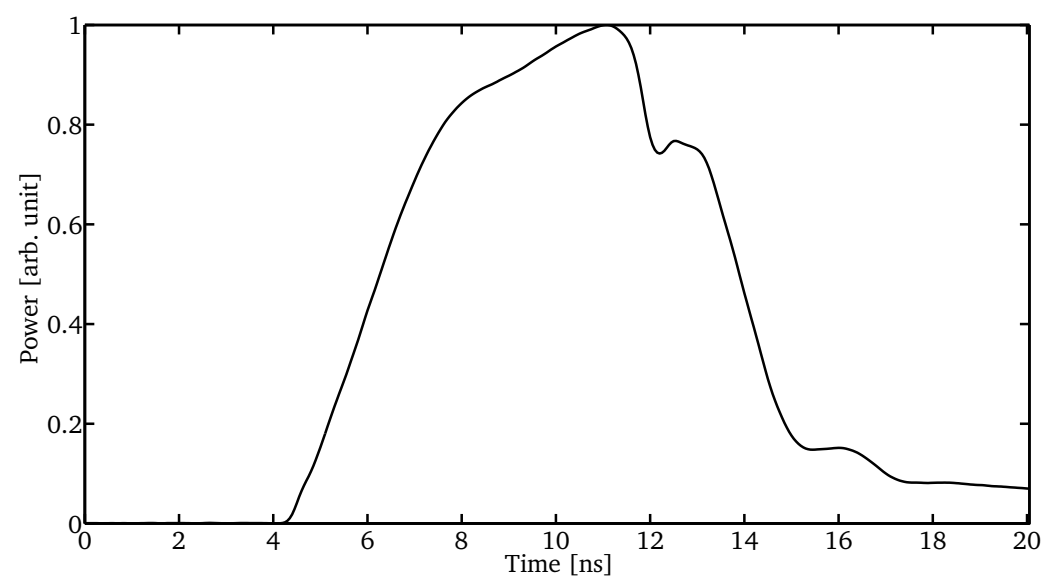

Figure 5.1: Measured temporal pulse shape of a 8 ns (FDHM) laser pulse emitted by the sPI laser source.

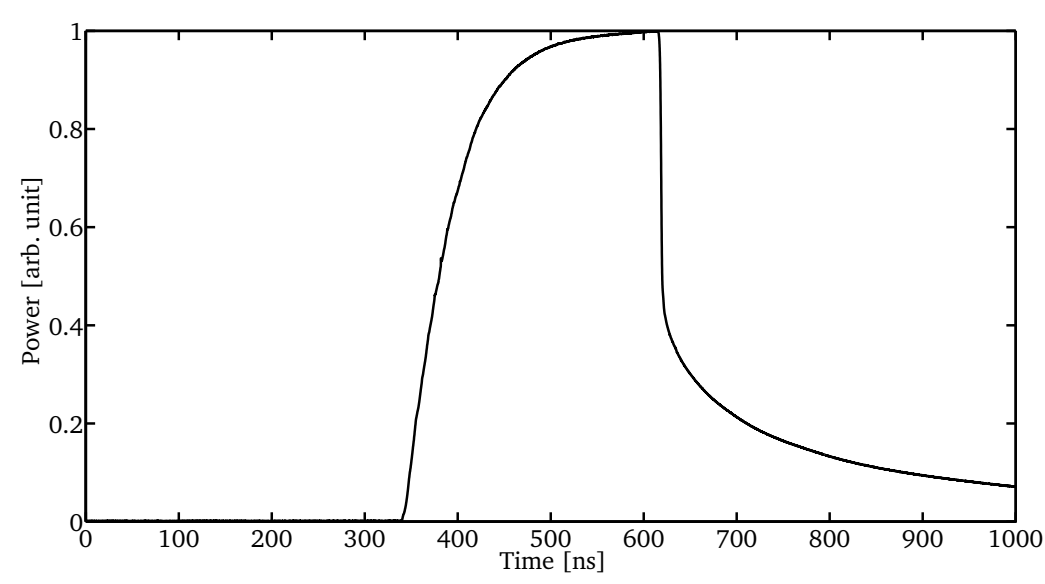

Figure 5.2: Measured temporal pulse shape of a 240 ns (FDHM) laser pulse emitted by the SPI laser source. 


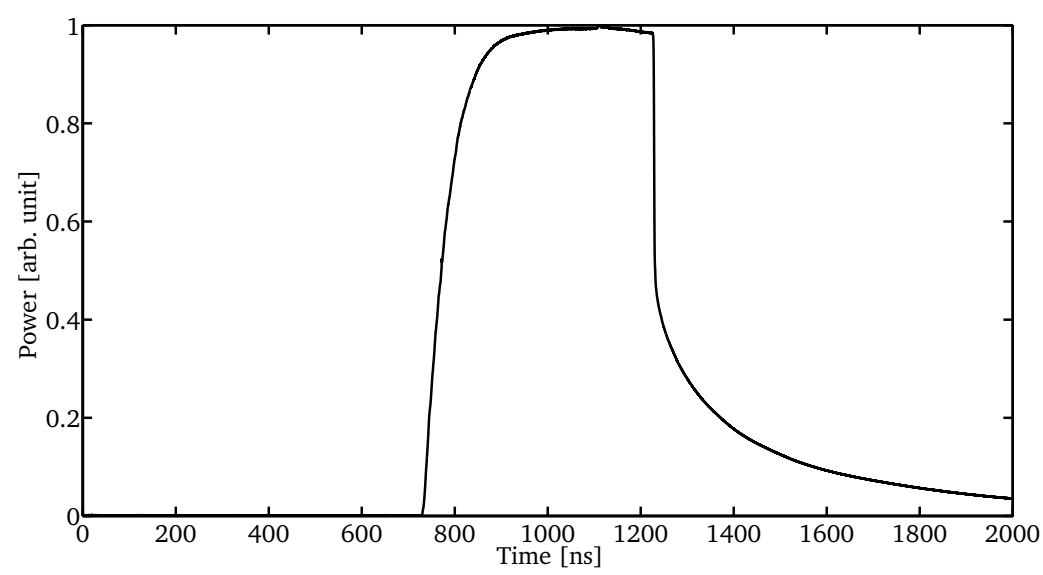

Figure 5.3: Measured temporal pulse shape of a 460 ns (FDHM) laser pulse emitted by the SPI laser source.

Table 5.2: List of components used in the experimental set-up.

\begin{tabular}{lll}
\hline Component & 1064 nm wavelength & 1550 nm wavelength \\
\hline $\begin{array}{l}\text { Polarizing beamsplitter } \\
\text { Beam attenuator }\end{array}$ & $\begin{array}{l}\text { Thorlabs CM1-PBS253 } \\
\text { Thorlabs VBA05-1064 }\end{array}$ & $\begin{array}{c}\text { Thorlabs CM1-PBS254 } \\
\text { Thorlabs VBA05-1550 } \\
\text { Camera }\end{array}$ \\
& Thorlabs BB1-E03P & Thorlabs BSX12 \\
Thaging lens & & App. Scin. Tech. \\
Translation axes & Thorlabs LA1257-C \\
DC servo motors & Thorlabs RB13M/M \\
Motor controllers & Thorlabs Z812B \\
Rotation axes & Thorlabs TDC001 \\
\hline
\end{tabular}




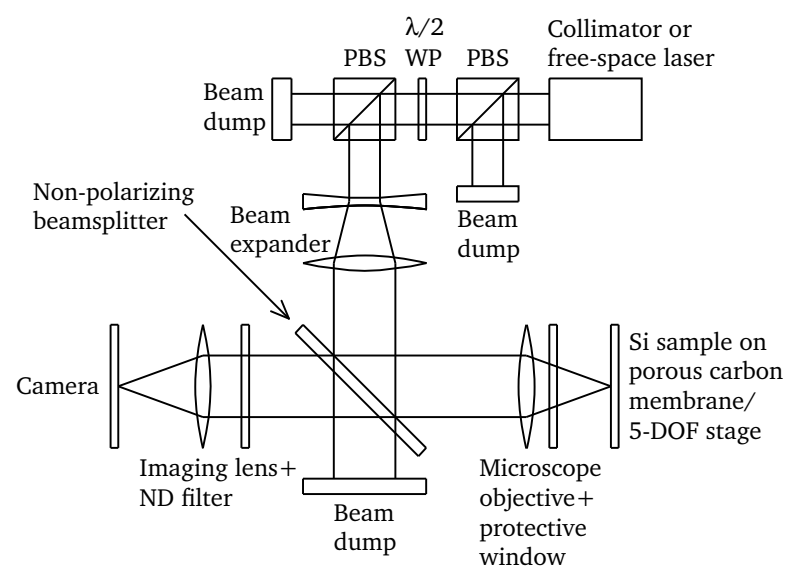

Figure 5.4: Drawing of the experimental set-up. Abbreviations used in the figure: PBS: polarizing beamsplitter, ND: neutral density, DOF: degrees of freedom, WP: waveplate.

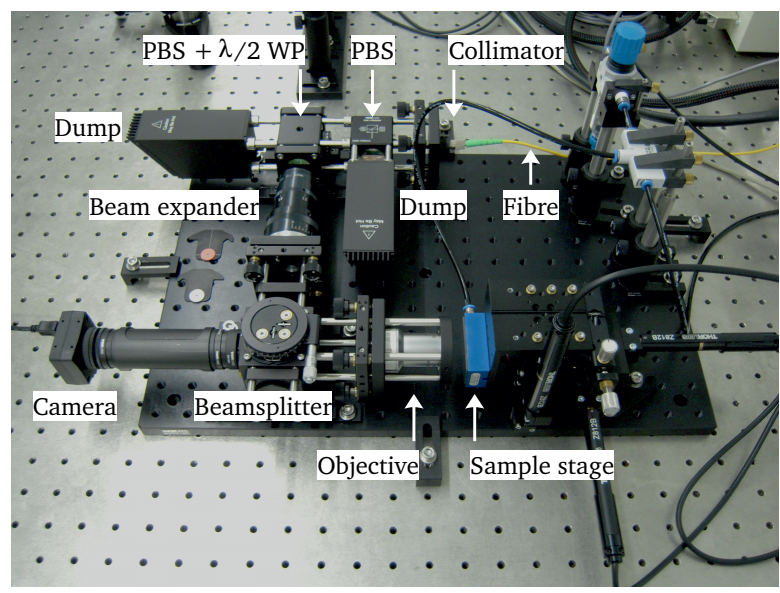

Figure 5.5: Photograph of the experimental set-up attached to the $1549 \mathrm{~nm}$ laser source. Abbreviations used in the figure: PBS: polarizing beamsplitter, WP: waveplate.

sources were terminated by SPI PT-POO577 and Thorlabs F24OAPC-155 collimators. As these lasers emit randomly polarized light, the collimated beams were subsequently polarized by polarizing beamsplitters.

Next, in order to obtain the desired pulse energy, the laser beam was attenuated by a half-lambda waveplate and another polarizing beamsplitter. The Spectra Physics laser source emits high energy pulses. Therefore, an additional attenuation step had to be incorporated after this source to prevent damage to the components located further along the beam path. This attenuation step consisted of a glass window with an uncoated front surface and an anti-reflection coated back surface. Only the Fresnel reflections from the front surface were directed towards the rest of the set-up. 
After exiting the variable attenuator, the beam was vertically polarized. The laser beam was subsequently expanded to obtain the desired $1 / e^{2}$ beam diameter with respect to the back aperture of the microscope objective. The microscope objective provides a beam that is tightly focussed inside the silicon sample.

To calibrate the focus position and to correctly align the samples with respect to the objective, a non-polarizing beamsplitter was employed that transmits a small percentage of the light reflected from the silicon surface. A plano-convex lens was used to image the reflected laser spot on a camera chip. When the focus of the microscope objective was located on the sample surface, the reflected light was also in focus on the camera. To adjust the intensity of the spot to the requirements of the camera, an infrared neutral density filter was placed next to the imaging lens. For the $1064 \mathrm{~nm}$ wavelength, a silicon complementary metal-oxide-semiconductor (cMos) camera was employed. However, silicon sensors cannot detect light with a $1550 \mathrm{~nm}$ wavelength. For this wavelength, a silicon charge-coupled device (CCD) with an anti-stokes phosphor coating to upconvert the energy of the photons was selected.

The ability to position the samples with micrometre accuracy is essential, as a positioning error of one micrometre results in an error of the focus position inside the sample of $3.5 \mu \mathrm{m}$. This difference is due to the high refractive index of silicon. By measuring the positions of laser-induced modifications inside silicon samples after laser processing, it was found that the alignment error of the samples was below a micrometre.

The samples were mounted on a porous carbon membrane with a flatness below $500 \mathrm{~nm}$. A chamber behind this membrane was kept at a low vacuum by means of a venturi. The sample holder could be manipulated by a stage with five degrees of freedom. Three translation axes equipped with DC servo motors were available. Both rotation axes were manually controlled to ensure that the focal spot was always at the same depth inside the sample, when translating the sample horizontally or vertically. The translation axes are constructed using crossed roller bearings and provide a range of $13 \mathrm{~mm}$. The servo motors have a maximum speed of $2.3 \mathrm{~mm} / \mathrm{s}$ and a bidirectional repeatability below $1.5 \mu \mathrm{m}$ after backlash correction.

Power measurements The energy of the laser pulses was measured behind the focussing objective to obtain the on-sample pulse energy. For this purpose, a laser power meter consisting of a transimpedance amplifier (Thorlabs PM1OOA) and a measurement head (Thorlabs S132C) containing a Germanium photodiode and a neutral density filter were employed. The uncertainty in the power measurements is $\pm 5 \%$. Since the pulse repetition rate of the laser is known, the measured average power can be converted to an energy value per pulse. A narrow laser beam was employed for the power measurements to prevent errors due to the dependence of the sensor sensitivity on the angle of incidence. When extrapolating the data to larger beam widths, the aperture losses were taken into account.

\subsection{Microscope objective}

To tightly focus laser pulses inside silicon, a microscope objective was employed, which was manufactured by Leica Microsystems and has type number 11101666 . The specifications of 
this objective are listed in table $5 \cdot 3$. According to a transmission spectrum supplied by Leica Microsystems, the objective provides better than 90 percent transmission for wavelengths between 1050 and $1650 \mathrm{~nm}$, while proving a value close to 90 percent for a wavelength of $1030 \mathrm{~nm}$. Therefore, the objective is usable when combined with all laser sources listed in table 5.1 .

Table 5.3: Specifications of the Leica Microsystems 11101666 focussing objective.

\begin{tabular}{ll}
\hline Property & Value \\
\hline Back focal length & $\infty$ \\
Design wavelength & $1300 \mathrm{~nm}$ \\
Focal length & $3.27 \mathrm{~mm}$ \\
Working distance & $>2.49 \mathrm{~mm}$ \\
Back aperture & $6 \mathrm{~mm}$ \\
Numerical aperture & 0.7 \\
Magnification (1.6x tube lens) & $100 \mathrm{x}$ \\
Medium between objective and sample & air \\
Coverslip correction & $100 \mu \mathrm{m}$ silicon \\
\hline
\end{tabular}

The focussing objective has a numerical aperture (NA) of 0.7. This value is a compromise between different aspects of the subsurface modification process. A too low NA results in insufficient confinement of the laser energy absorption. On the other hand, higher numerical apertures result in a larger beam diameter at the surface of the wafer, implying that a larger area has to be free of structures. Moreover, the working distance of high NA objectives is generally limited. Small working distances have a number of disadvantages for the formation of subsurface modifications. First, it is impossible to focus deep inside bulk materials. Secondly, there is a high risk of contact between the objective and the sample in case of alignment errors. Finally, the risk of damage when inadvertently ablating material from the sample surface is increased. Based on the above, a large working distance objective was selected that provides a good balance between a sufficiently high NA and a large enough working distance.

The microscope objective is designed to work together with a tube lens. Parts of the corrections regarding the field curvature are not located in the objective but in the tube lens. Since only a single laser beam with a constant position and angle with respect to the back aperture of the objective was used during the experiments, the field curvature does not constitute a problem. Therefore, no tube lens was added to the experimental set-up.

Damage threshold measurements The microscope objective contains three lenses. Damage threshold data of these lenses were unavailable from the supplier. Therefore, damage tests were performed on uncoated samples of the lenses inside the objective, using the picosecond source listed in table 5.1. The pulse energy and average power 
were gradually increased during these tests. After each test, the lenses were optically inspected to check for damage. Up to a pulse energy of $125 \mu \mathrm{J}$ and an average power of $10 \mathrm{~W}$, no damage was found. As these conditions are far above the requirements for the process, it was concluded that the substrate material of the lenses is sufficiently resistant to laser-induced damage.

Since no samples of the lenses including an anti-reflection coating were available, the damage threshold of the coating could not be verified. No problems regarding coating damage were observed during experiments with pico- and nanosecond pulses using the complete objective. The damage threshold for nanosecond pulses will be substantially higher than for picosecond pulses, due to the lower light intensities that the lenses are exposed to. Since the successful production of subsurface modifications was only achieved when employing nanosecond pulses (see section 6.5), a reasonable safety margin is expected to be present for the processing conditions that were suitable for the subsurface modification process.

\subsubsection{Coverslip correction}

When focussing a laser beam inside a transparent medium using a high numerical aperture microscope objective, a coverslip correction matched to the focus depth and the refractive index of the medium has to be applied, to prevent spherical aberrations.

As indicated in table 5.3, the microscope objective has a fixed coverslip correction for $100 \mu \mathrm{m}$ of silicon. Consequently, if the focus depth deviates from the ideal value, aberrations will occur. In this section, the cause of the aberrations will be discussed, followed by quantitative computations to assess the magnitude of these aberrations.

Coverslip correction based on ray optics The reason why a coverslip correction is necessary can be understood from the refraction of optical rays based on Snell's law. The refraction of a single ray is shown in figure 5.6. When a ray propagating through a medium with index $n_{1}$ goes through an interface with a medium with index $n_{2}$, the corresponding angles with the optical axis $\theta_{1}$ and $\theta_{2}$ change according to [158]

$$
\frac{\sin \left(\theta_{2}\right)}{\sin \left(\theta_{1}\right)}=\frac{n_{1}}{n_{2}} \text {. }
$$

To evaluate what happens when the focus location is moved into the medium with index $n_{2}$, a lens is assumed that was designed such that all rays cross the optical axis in a single point, when focussing in a medium with index $n_{1}$. The working distance between the lens and the interface between both media is selected such that the focus is located at the interface. To place the focus inside the medium with index $n_{2}$, the working distance has to be reduced by $\Delta$ wd. Consequently, the focus will be shifted inside the material with index $n_{2}$ by a distance $d_{\text {foc }}$. The intercept height $h$ of the ray at the interface (see figure 5.6), can be connected to $\theta_{1}$ and $\Delta$ wd by

$$
h=\tan \left(\theta_{1}\right) \Delta \mathrm{wd} .
$$

The actual focus depth $d_{\text {foc }}$, in the presence of refraction, is related to $h$ by

$$
h=\tan \left(\theta_{2}\right) d_{\text {foc }} \text {. }
$$




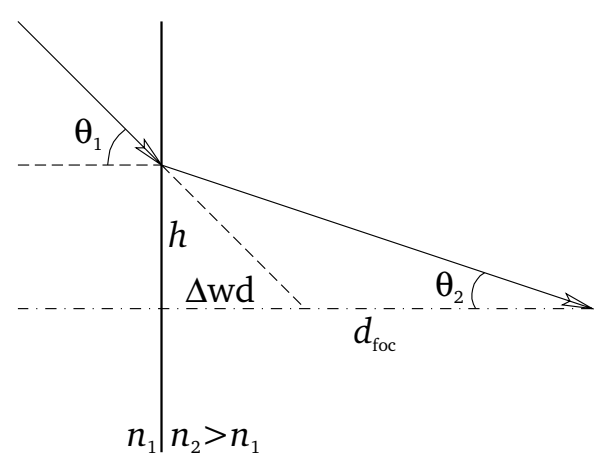

Figure 5.6: Drawing of the refraction of an optical ray, when propagating from a medium with refractive index $n_{1}$ into a medium with refractive index $n_{2}$. The drawing corresponds to a value of $n_{2}$ that is greater than $n_{1}$.

Substitution of equation (5.2) in equation (5.3) gives

$$
d_{\text {foc }}=\frac{\tan \left(\theta_{1}\right)}{\tan \left(\theta_{2}\right)} \Delta \mathrm{wd} .
$$

The problem that arises here is that according to equation 5.1), the ratio between the sines instead of the tangents of $\theta_{1}$ and $\theta_{2}$ is fixed, implying that the ratio between $d_{\text {foc }}$ and $\Delta \mathrm{wd}$, as given by equation 5.4 , is not. Consequently, rays arriving at the interface at different angles, cross the optical axis at different locations. For small angles, this does not constitute a practical problem $\operatorname{since} \sin (\theta) \approx \tan (\theta)$ for $\theta \approx 0$. Therefore, objectives with a low NA do not require corrections adapted to the focus depth and the refractive index of the medium. For high NA objectives, spherical aberrations will occur when corrections are absent (see figure 5.7).

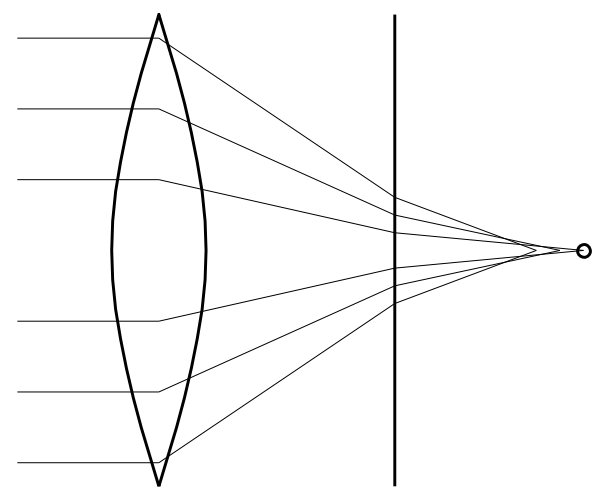

Figure 5.7: Drawing of the negative spherical aberrations that occur when focussing a laser beam inside a material with a refractive index greater than the medium that the objective was designed for, without applying a coverslip correction. Different rays do not cross the optical axis at the same point. 
Coverslip correction based on electromagnetic optics To quantitatively assess the effect of a mismatch in the coverslip correction, ray tracing cannot be employed due to the small size of the focal spot compared with the wavelength of the light. Moreover, the Gaussian laser beam profile and its scaling with respect to the aperture of the objective have to be considered. The beam profile affects the impact of a mismatch in the coverslip correction, as it determines the distribution of power between the paraxial and peripheral rays. Therefore, simulations have been run using the PSF Lab software [159] (see section 4.2). The PSF Lab software computes the required coverslip correction for a certain design case and evaluates the effect of specific deviations from the design conditions.

In figure 5.8, the laser intensity profile for the ideal case is shown, in which the laser beam is focussed $100 \mu \mathrm{m}$ below the surface, which matches the coverslip correction. The conditions were identical to those used in section 4.2. In this case, the intensity profile is symmetric around the focus.

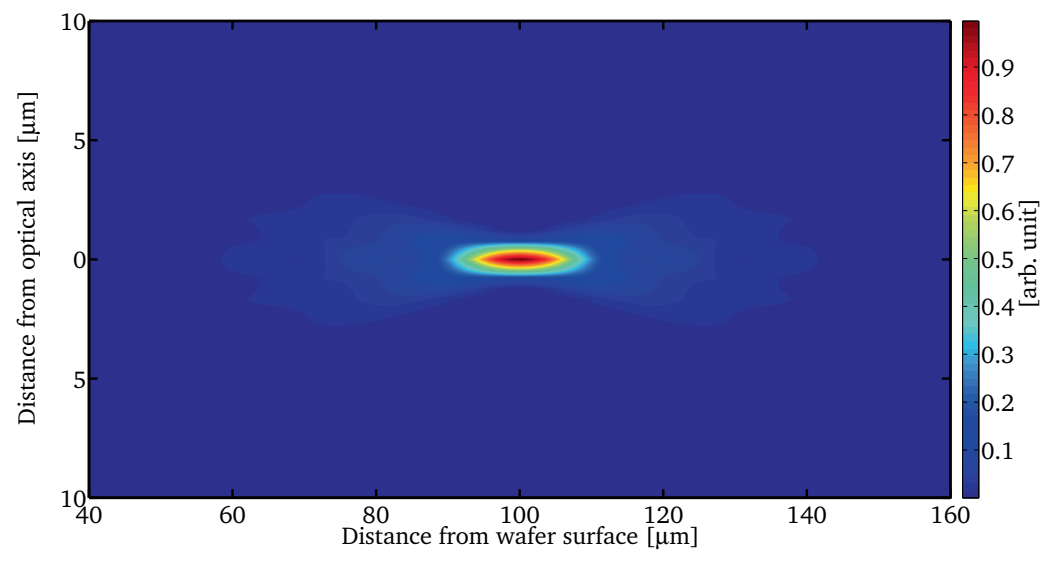

Figure 5.8: Cross-section of the laser intensity distribution [arbitrary unit] in the bulk of silicon, based on an electromagnetic wave that satisfies Maxwell's equations. The coverslip correction of the objective was matched to the refractive index of silicon and the focus depth. Wavelength: $1064 \mathrm{~nm}$. The beam propagation direction is from left to right.

During some experiments, the objective was protected by a quartz window to prevent damage when material was ablated from the surface. Ablation may happen inadvertently when an incorrect focus depth or pulse energy is selected. It may also be required to produce surface alignment marks (see section 5.5). In figure 5.9. a focus depth of $100 \mu \mathrm{m}$ has been simulated in the presence of a $250-\mu \mathrm{m}$ thick quartz window. Here, negative spherical aberrations are visible resulting in a larger depth of focus. The intensities have been scaled, such that the peak intensity occurs at a value of one. When comparing the unscaled values of the intensities, it was found that the peak intensity in the focus is reduced by a factor two compared with the ideal case, due to the spherical aberrations caused by the presence of the quartz window.

For the current research, $160-\mu \mathrm{m}$ thick wafers were used (see section 6.2). Therefore, the largest deviation from the design value of the coverslip correction, without the presence of a protective window, would occur when the beam is focussed close to the surface. In 


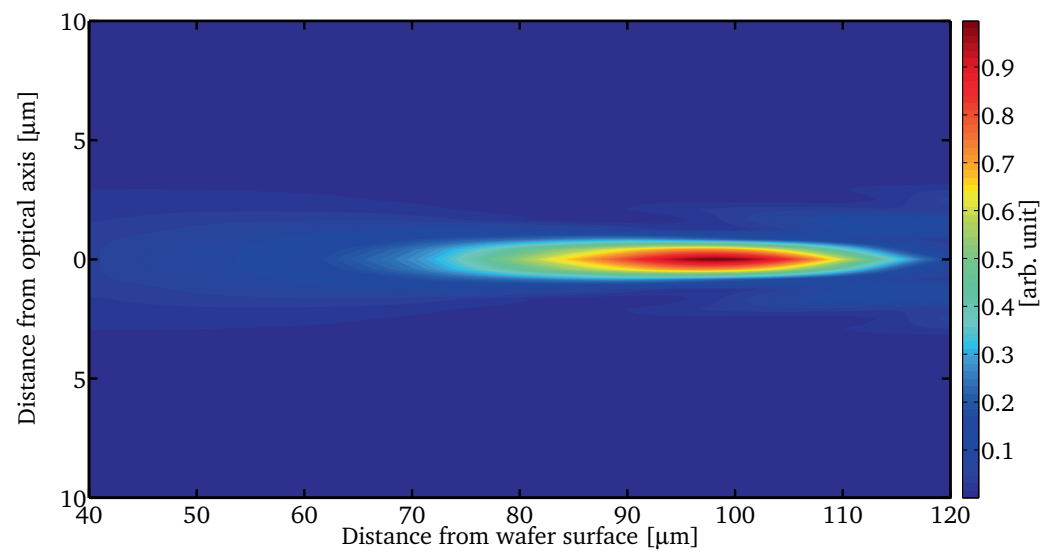

Figure 5.9: Cross-section of the laser intensity distribution [arbitrary unit] in the bulk of silicon, based on an electromagnetic wave that satisfies Maxwell's equations. The coverslip correction of the objective and the focus were fixed at $100 \mu \mathrm{m}$ while an additional $250-\mu \mathrm{m}$ thick quartz window was present. Wavelength: $1064 \mathrm{~nm}$. The beam propagation direction is from left to right. Negative spherical aberrations are visible.

figure 5.10, a focus depth of $20 \mu \mathrm{m}$ has been simulated. Since in this case the actual silicon cover is less than the design value, positive spherical aberrations can be observed. Nevertheless, the aberrations are less severe than in the presence of a $250-\mu \mathrm{m}$ thick quartz window. The decrease in peak intensity was approximately 15 percent.

\subsection{Sample geometry}

The repetition rate of the laser sources was fixed at 10 or $100 \mathrm{~Hz}$ during the production of subsurface modifications. By varying the speed of the stage, the spacing between the subsurface modifications was adjusted. At $100 \mathrm{~Hz}$, a pulse-to-pulse spacing up to $23 \mu \mathrm{m}$ could be obtained, within the maximum speed of the stage (see section 5.3). If required, the generation of pulses by the laser source was enabled or disabled to limit the production of modifications to specific locations.

When modifications were produced at different focus depths, the deepest modifications were created first, to preclude the laser beam from being affected by previously created modifications. To prevent the process from being influenced by absorption of laser energy by the sample holder, a layer of transparent tape was attached to the back surface of the samples.

Four different sample geometries were produced:

a. Samples containing multiple layers of closely spaced modifications for the purpose of wafer dicing (see figure 5.11a).

b. Samples containing one or more layers of closely spaced modifications, combined with a layer of modifications with a large spacing between the pulses (see figure 5.11b). 


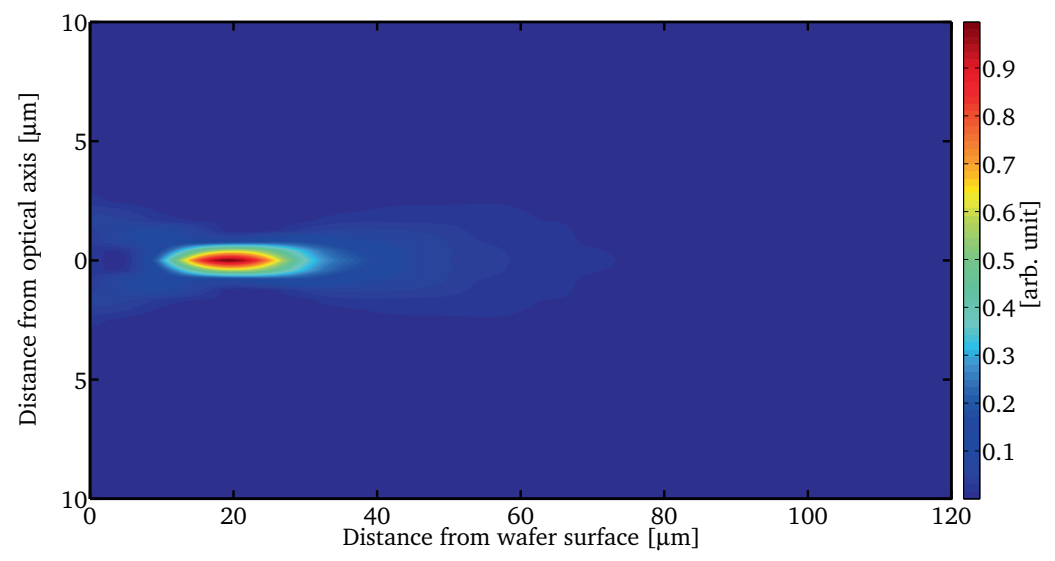

Figure 5.10: Cross-section of the laser intensity distribution [arbitrary unit] in the bulk of silicon, based on an electromagnetic wave that satisfies Maxwell's equations. The coverslip correction of the objective was fixed at $100 \mu \mathrm{m}$ while the focus was located at a depth of $20 \mu \mathrm{m}$. Wavelength: $1064 \mathrm{~nm}$. The beam propagation direction is from left to right. Positive spherical aberrations are visible.

This geometry allows for the samples to be diced, while the cross-sections still contain single-pulse modifications that can be subject to further analysis.

c. Samples containing modifications with a large spacing between the pulses for the analysis of single-pulse modifications (see figure 5.11c). Laser-induced surface marks may be needed to localize the subsurface modifications.

d. Samples containing streets with dense modifications in the vicinity of streets containing modifications that are to be analysed (see figure 5.11d). These samples are designed to fracture along the dense modifications. Failure of the samples along the plane with the modifications that are intended for analysis should be prevented.

When samples of geometry type (c) or (d) are subject to further analysis based on surface analysis methods, additional material removal techniques have to be applied to expose the modifications (see section 5.7).

\subsection{Non-destructive analysis tools}

When investigating subsurface damage in silicon, non-destructive analysis methods are preferred, as they do not introduce additional uncertainty regarding the cause of the observed damage. Moreover, problems with locating the subsurface modifications are avoided. Two methods to detect damage in a non-destructive manner were evaluated: infrared microscopy and X-ray computed tomography. 

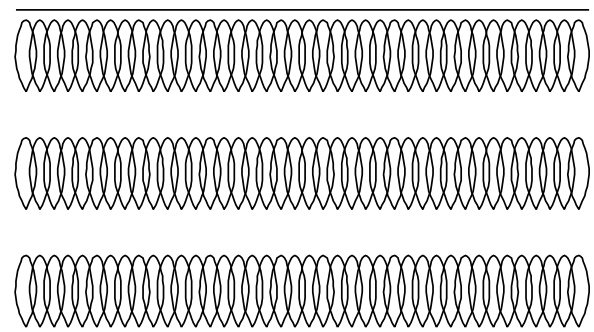

(a) Sample geometry type (a).

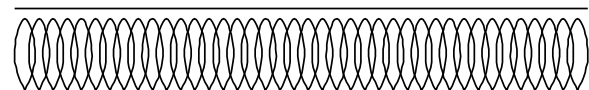

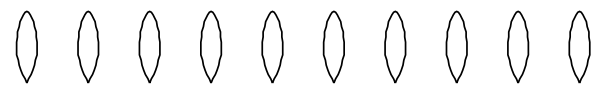

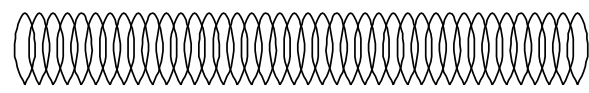

(b) Sample geometry type (b).

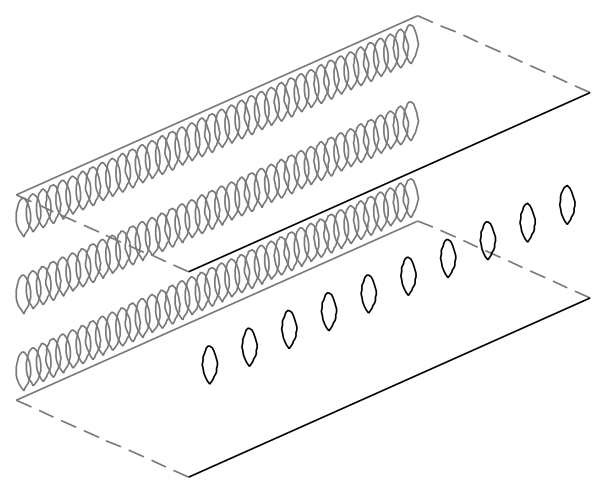

(d) Sample geometry type (d). (c) Sample geometry type (c).

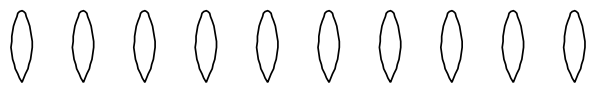

Figure 5.11: Cross-sections corresponding to different sample geometries. Each elliptic contour represents a subsurface modification. The laser beam propagation direction is from top to bottom.

\section{Infrared microscopy}

To establish whether subsurface modifications were present inside silicon, a Leica DMRM microscope equipped with an infrared light source below the sample stage was employed. This infrared source consists of a halogen light bulb and a longpass filter. For the observation of the transmitted light, a silicon cmos camera was used (IDS uEye $1240 \mathrm{ME}$ ). The infrared blocking filter in this camera was replaced by uncoated float glass. As both the sample and sensor were made of silicon, a narrow wavelength range around one micrometre, where the sample becomes transparent while the camera still provides some sensitivity, was imaged.

When comparing images obtained by infrared transmitted light with images recorded using visible light reflected from the surface, it was found to be possible to identify whether the damage was located on or below the surface. Subsurface damage that is not visible using reflected visible light, showed up as a dark spot when employing infrared illumination. For the observation of the sample surface, both bright- and darkfield illumination were employed.

As surface damage is also visible in infrared, it has to be ruled out before concluding that a modification is located below the surface. By comparing geometric profile data 
with images obtained using optical microscopy, it was found that optical detection is the most sensitive method to detect the first signs of inadvertent surface damage. The same conclusion was drawn in reference [64].

Further, it was found that due to the small wavelength range that was imaged during infrared transmission measurements, even objectives that are only corrected for chromatic aberrations in the visible spectrum, provide an acceptable image quality. The best image quality and light transmittance were obtained when employing the same objective as used for the machining of the samples (Leica Microsystems 11101666 , see section 5.4). The level of detail that can be resolved by infrared microscopy is inferior compared with regular optical microscopy using visible light. While it is possible to identify the presence of subsurface modifications, limited information about their shape can be extracted.

\section{X-ray computed tomography}

The University of Leuven (Belgium) has conducted an initial feasibility study using samples produced during this work, regarding the application of a computed tomography (Ст) scanner to analyse subsurface modifications in silicon. In a CT-scan, contrast is generated by differences in X-ray absorption, which is mainly related to the density of the material. A General Electric Nanotom scanner was used for the feasibility study. This scanner is capable of imaging voxels with a size of $500 \mathrm{~nm}$. Due to the noise that is present in the images, the smallest detectable feature is somewhat larger than the voxel size.

A 2 by $3 \mathrm{~mm}$ silicon sample containing subsurface modifications was placed in front of the X-ray source. By rotating the sample, images were recorded at different angles to reconstruct a three-dimensional image. No contrast was observed in the images obtained from the CT-scan. This implies that any voids that are present in the material are smaller than the minimum detectable feature size of the cT-scanner.

\subsection{Destructive analysis tools}

While infrared microscopy was found to be suitable to detect the presence of laser-induced subsurface modifications in silicon, it has limitations with respect to the information about the shape and size of the modifications that can be obtained. To acquire detailed information about the geometry and material structure of subsurface modifications, destructive methods were employed. One of the main challenges when preparing samples for further analysis is to locate the micrometre-sized subsurface modifications, which are hidden inside the bulk of the material. In this section, the destructive sample preparation methods will be discussed, followed by the analysis techniques that were employed after the sample preparation.

\subsubsection{Sample preparation}

The purpose of destructive sample preparation techniques is to expose subsurface modifications on a surface, or to obtain thin lamellae that contain cross-sections of the modifications. Three preparation methods have been used, which are listed below. 


\section{Laser-induced subsurface separation}

Subsurface modifications were used to fracture silicon samples along a predefined plane. Any features present on this plane may then be analysed using surface analysis methods. Sample geometry types (a) and (b) (see section 5.5) were employed for this preparation technique. Laser-induced subsurface separation is the most efficient sample preparation method. A disadvantage of this approach is that the fracture planes have a roughness of a few hundred nanometres (see section 6.7).

\section{Focussed ion beam milling}

To create cross-sections of subsurface modifications along the optical axis, focussed ion beam (FIB) milling was applied. For the coarse removal of material a FEI 200TEM single beam system was used, while for nanometre-scale processing a FEI Helios dual beam system consisting of a FIB and a scanning electron microscope (SEM) was employed. The resulting lamellae are electron-transparent. Sample geometry type (c) (see section 5.5) was used for this preparation technique. Additionally, surface marks were produced by the laser in the vicinity of the subsurface modifications, to start the ion milling procedure at the correct location.

\section{Mechanical polishing}

To create cross-sections perpendicular to the optical axis, samples were first polished to approach the modifications within a few micrometres. Next, electron-transparent lamellae were produced by a FEI Helios 60o NanoLab FIB at specific locations along the optical axis. Sample geometry type (d) (see section 5.5) was used for this preparation technique. The advantage of this sample geometry is that a plane formed by fracturing the sample, which is parallel to the plane containing the modifications that are to be analysed, is available. This plane was used as a starting plane for the polishing procedure.

\subsubsection{Surface and lamella analysis tools}

After exposing subsurface modifications on a surface or in a thin lamella, several analysis techniques can be applied. The results of these analyses are presented in chapters 6 and 7 The analysis techniques that were applied are listed below.

\section{Optical microscopy}

For the analysis of fracture planes with moderate resolution, a Leica DMRM microscope equipped with a cmos camera was employed. For each objective lens, the distance corresponding to the camera pixels was calibrated using a calibration slide to enable quantitative distance measurements.

\section{Laser scanning confocal microscopy (LSCM)}

To analyse fracture planes with higher resolution and depth of focus compared with optical microscopy, a laser scanning confocal microscope (Keyence vk-9710) was employed. This microscope contains a $408 \mathrm{~nm}$ ultraviolet laser source. Two types of analyses were performed. First, integrated intensity images were recorded which resemble brightfield optical microscopy images. Secondly, by measuring the sample height corresponding to the peak intensity for each pixel, height maps were produced to estimate the roughness of the fracture planes. 


\section{Scanning electron microscopy (SEM)}

A Jeol JSM-6400 scanning electron microscope was used to obtain the most detailed micrographs of fracture planes. Since secondary electrons were measured, contrast is mainly generated by differences in surface angle.

\section{Raman spectroscopy}

To identify the material structure of silicon in a thin layer below the surface, a Renishaw 2000 Raman spectroscopy system equipped with a HeNe laser source emitting at $633 \mathrm{~nm}$ was employed. By comparing the wavelengths of inelastically scattered light with known reference data, the presence of specific phases can be detected [165]. The diameter of the spot of the Raman microscope is approximately $2 \mu \mathrm{m}$, so data is collected from a relatively large area. The absorption coefficient of diamond cubic silicon at $633 \mathrm{~nm}$ is $3.75 \cdot 10^{5} 1 / \mathrm{m}[166$, resulting in a $1 / e$ optical penetration depth of approximately $2.6 \mu \mathrm{m}$.

\section{Transmission electron microscopy (TEM)}

For the analysis of electron-transparent lamellae with nanometre resolution, a Philips Tecnai, a Phillips CM300 and a Jeol JEM-210of transmission electron microscope were used. In addition to bright- and darkfield micrographs in conventional TEM mode, scanning transmission electron microscopy (STEM) images were obtained using a high-angle annular darkfield (HAADF) detector. Advantage of STEM compared with conventional TEM is that bend contours are suppressed [167], allowing the shape of the subsurface modifications to be clearly identified. Moreover, it is less affected by the sample thickness. Additionally, selected area diffraction (SAD) patterns were recorded, to identify the material phases that were present in specific parts of the laser modifications.

\subsection{Summary}

An experimental set-up was developed to focus infrared laser pulses with wavelengths around 1064 and $1550 \mathrm{~nm}$ inside silicon samples. This set-up is capable of aligning the position of the samples along the optical axis with micrometre accuracy. The sample position is controlled by three automated translation axes. Both the energy of the laser pulses and the beam diameter are adjustable. An infrared microscope objective with a numerical aperture of 0.7 and a working distance of $2.5 \mathrm{~mm}$ was selected to focus the laser beam. This microscope objective contains corrections to prevent spherical aberrations for a focus depth of $100 \mu \mathrm{m}$ inside silicon.

The laser pulses were generated by four laser sources. Three sources provide photons with an energy close to the band gap of silicon. These lasers have pulse durations of $6.6 \mathrm{ps}, 8 \mathrm{~ns}$ and 2-46o ns and wavelengths of 1030, 1064 and $1061 \mathrm{~nm}$ respectively. The fourth source generates pulses of $3.5 \mathrm{~ns}$ with a longer wavelength of $1549 \mathrm{~nm}$, resulting in a photon energy below the band gap. The laser with a tunable pulse duration allows for the instantaneous power of the pulses to be varied, to enable or disable two-photon absorption. Two of the laser sources are fibre lasers. Fibre lasers are well suited for the subsurface modification process, as they provide a sufficiently high pulse energy and have an excellent beam quality. All lasers were configured for low repetition frequencies, either 
by pulse picking or pulsed pumping. Therefore, separate single-pulse modifications can be generated.

Four different sample geometries were proposed that are adapted to various nondestructive or destructive analysis techniques. To detect the presence of subsurface modifications in a non-destructive manner, infrared microscopy was found to be suitable. No contrast was observed when analysing subsurface modifications using a CT-scan. To obtain detailed information about the geometry and crystal structure of modifications, methods to expose the subsurface modifications on a surface or in a lamella were proposed. Afterwards, optical microscopy, laser scanning confocal microscopy, Raman spectroscopy, scanning electron microscopy or transmission electron microscopy can be employed. 


\section{Experimental results: geometry}

In this chapter, ${ }^{*}$ the geometries of subsurface modifications, which were produced using the experimental set-up described in chapter 5, will be presented. The geometries are compared with numerical predictions to validate the numerical model. Additionally, the modification mechanisms and the suitability of the modifications for wafer dicing will be considered.

\subsection{Introduction}

This chapter is focussed on the experimental investigation of the geometry of laser-induced subsurface modifications. The analysis of the crystal structure of the modifications can be found in chapter 7. During the experimental studies, the influence of the pulse duration, pulse energy and wavelength on the shape of the subsurface modifications was investigated. Based on the results from numerical simulations (see chapter 4), these parameters are expected to have the largest influence on the outcome of the laser process.

An important goal of the experimental data is to validate the numerical model. Therefore, the geometries of the subsurface modifications will be compared with simulation results, to assess the accuracy of the numerical model under various processing conditions. Moreover, the suitability of the subsurface modifications for wafer dicing will be discussed, including the mechanisms that contributed to the separation of the dies. Additionally, the information that the fracture planes provide regarding the modification mechanisms that occurred during the laser-material interaction will be considered.

\subsection{Experimental conditions}

The wafers that were selected for the experiments are $160-\mu \mathrm{m}$ thick monocrystalline silicon wafers with a $<100>$ crystal orientation and a moderate boron doping. The silicon wafers were of a high quality grade that is suited to the production of integrated circuits. The resistivity of one of the wafers was measured by the supplier using a Prometrix Omnimap Rs75 resistivity mapping system. The measured resistivity of $10.3 \Omega \cdot \mathrm{cm}$ is sufficiently high to prevent significant free carrier absorption of laser energy under equilibrium conditions [58]. The experiments were performed at a controlled ambient temperature of $293 \mathrm{~K}$.

Entire wafers are too large to be handled by the experimental set-up, as it can only translate samples by up to $13 \mathrm{~mm}$ (see section 5.3). Therefore, the wafers were pre-diced to form samples measuring 20 by $5 \mathrm{~mm}$. During the dicing tests, the samples were split in half resulting in two dies of 10 by $5 \mathrm{~mm}$. The dicing plane was approximately aligned with a $\{110\}$ plane.

*Experimental results from the $1549 \mathrm{~nm}$ process have been published in Optics Express, 22(18):21958-21971, 2014 (P. C. Verburg, G. R. B. E. Römer, and A. J. Huis in 't Veld). 
When quantitative measurements were performed, the focal spot was located at a depth of $100 \mu \mathrm{m}$ inside the silicon and no window was placed behind the objective lens. This ensures that no spherical aberrations are present (see section 5.4.1). During all other experiments, unless mentioned otherwise, a $250-\mu \mathrm{m}$ thick quartz window was present behind the objective to protect it against damage. This window induces negative spherical aberrations, which have an effect on the shape of the modifications.

The beam diameter at the back aperture of the objective was chosen such that the $1 / e^{2}$ diameter fills 80 percent of the aperture. This choice results in a sufficiently small focal spot inside silicon, while preventing excessive energy losses or diffraction effects (see section 4.2). An overview of the resulting spot sizes in silicon for the different laser sources can be found in table 6.1. These sizes were computed based on non-paraxial computations using the PSF Lab software [159] (see section 4.2.)

Table 6.1: Theoretical spot size inside silicon for the different laser sources. The focus was assumed to be located $100 \mu \mathrm{m}$ below the wafer surface, which matches the coverslip correction of the microscope objective. The spot size of the Spectra Physics source could not be computed as its beam profile is undefined.

\begin{tabular}{llll}
\hline $\begin{array}{l}\text { Manufacturer and } \\
\text { type }\end{array}$ & Wavelength & $\begin{array}{l}\text { Beam quality } \\
\left(M^{2}\right)\end{array}$ & $\begin{array}{l}\text { Spot size } \\
\left(1 / e^{2} \text { diameter }\right)\end{array}$ \\
\hline $\begin{array}{l}\text { Trumpf } \\
\text { Trumicro 5050 }\end{array}$ & $1030 \mathrm{~nm}$ & 1.3 & $1.84 \mu \mathrm{m}$ \\
\hline $\begin{array}{l}\text { Spectra Physics } \\
\text { Quanta Ray GCR-270 }\end{array}$ & $1064 \mathrm{~nm}$ & - & - \\
\hline $\begin{array}{l}\text { SPI } \\
\text { SP-020P-A-EP-S-A-Y }\end{array}$ & $1061 \mathrm{~nm}$ & 1.3 & $1.92 \mu \mathrm{m}$ \\
\hline $\begin{array}{l}\text { MWTechnologies } \\
\text { MOPA-LF-1550 }\end{array}$ & $1549 \mathrm{~nm}$ & 1.1 & $2.37 \mu \mathrm{m}$ \\
\hline
\end{tabular}

\subsection{Model validation for surface damage thresholds}

The quantitative measurements of the geometry of the subsurface modifications were based on a destructive sample preparation method (see section 6.4). Since destructive analysis techniques impose a risk of causing further damage beyond the damage induced by the laser-material interaction, the performance of the model has first been verified with experimental data on surface instead of subsurface damage thresholds. Not all aspects of the model, including the beam propagation inside the material, can be validated in this manner. Nevertheless, a comparison between simulated and measured surface damage thresholds allows for the mechanisms of laser energy absorption to be validated. Moreover, surface damage thresholds can be reliably measured and are readily available in literature. 
The numerical predictions of surface damage were based on simulations of a laser beam with a Gaussian power density profile interacting with a silicon substrate. The temporal profile of the laser pulses was also assumed to be Gaussian (see section 3.3.1). By measuring the radial extent of the molten material during post-processing, the required fluence (energy per unit area) to damage the silicon surface was established.

The experimental data on surface modification thresholds were collected from literature on damage observed by optical microscopy or reflectivity measurements for picosecond [168], $10 \mathrm{~ns}[76,169], 30 \mathrm{~ns}$ [170] and $40 \mathrm{~ns}$ pulses [74], all at wavelengths ranging from 1060 to $1064 \mathrm{~nm}$.

The comparison of the simulation results with experimental data is presented in figure 6.1 The vertical error bars show, if available, the combined ranges of o to 100 percent damage probability, compiled from the references stated above. The data markers indicate the midpoint of this range. An excellent agreement between the numerical and experimental results is observed.

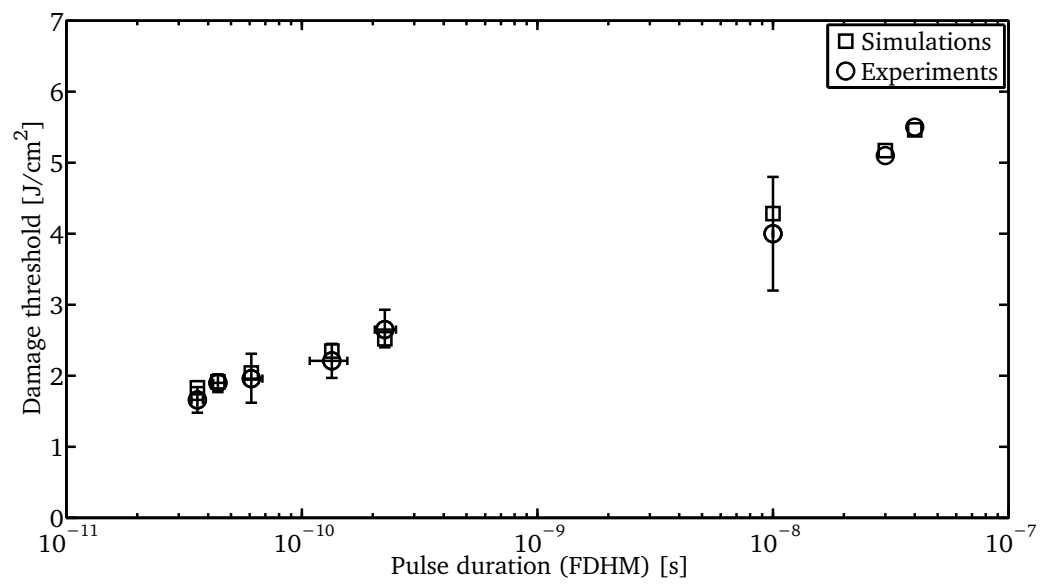

Figure 6.1: Comparison of experimental and simulated surface damage thresholds. Wavelength: $1064 \mathrm{~nm}$. The experimental data were obtained from references $[74,76,168,-170]$.

According to the numerical simulations, the main reason for the decrease in surface damage thresholds for shorter pulse durations was found to be the increasing contribution of two-photon absorption. Since linear absorption at a wavelength of $1064 \mathrm{~nm}$ is not very effective at room temperature, two-photon absorption at high laser intensities helps to absorb the laser energy in a thin surface layer.

\subsection{Measurement of the modification threshold and geometry}

The aim of the sample analyses that are presented in this chapter is twofold. First, the existence of subsurface modifications has to be established as a function of the relevant laser processing conditions. For the conditions that were found to result in modifications, 
the length of the subsurface modifications along the optical axis was measured. The analysis methods that were employed for both purposes are discussed below.

Measurement of the existence of modifications To establish whether subsurface modifications are present, infrared microscopy (see section 5.6) was employed, based on sample geometry type (c) (see section 5.5). The main advantage of infrared microscopy is that it is non-destructive. Consequently, it can be stated with certainty that the features that are observed on infrared micrographs are a direct result of the laser process.

To ensure that the presence of modifications is reliably detected, even when their visibility is poor, laser-induced surface marks were created in the vicinity of the locations where the laser pulses were focussed inside silicon. In this manner, the locations where subsurface modification might be present are precisely known during the sample analysis.

An example of this type of measurement is shown in figure 6.2. A reflected light brightfield micrograph and an infrared transmission micrograph, corresponding to the same location, are shown. Both the subsurface modifications and the surface marks are visible in infrared, while the visible light only shows the surface marks. Some debris originating from ablated material is visible which originated from the formation of these marks. To prevent this material from interfering with the propagation of the laser beam into the sample, the marks were created after the production of the subsurface modifications.

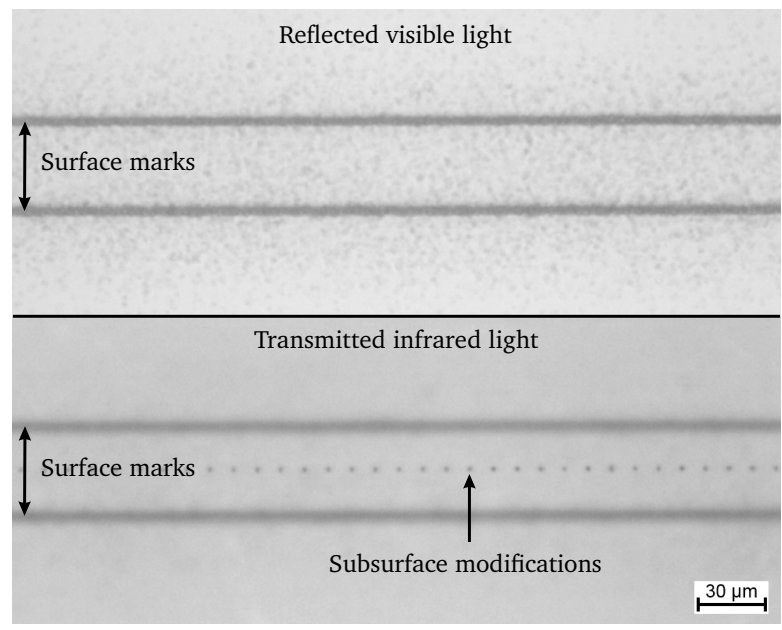

Figure 6.2: Optical (top) and infrared (bottom) micrographs of a silicon sample containing subsurface modifications spaced $10 \mu \mathrm{m}$ apart, located between two surface marks. Wavelength: $1549 \mathrm{~nm}$, pulse energy: $1.3 \mu \mathrm{J}$, pulse duration: $3.5 \mathrm{~ns}$, focus depth: $70 \mu \mathrm{m}$ below the surface.

To measure the thresholds for the formation of subsurface modifications, laser pulses were focussed inside silicon samples along numerous tracks. For each track, different processing conditions were selected. Based on which tracks contained visible spots, the process boundaries were established. 
Measurement of the modification geometry While infrared microscopy is suitable to determine whether or not modifications are present, it does not provide accurate data concerning their geometry. To measure the length of the subsurface modifications along the optical axis, a destructive analysis method based on fracturing was employed (see section 5.7.1). The modification length was also used to parametrise the geometry of the modifications during the numerical simulations (see section 4.5.1). Sample geometry type (b) (see section 5.5) was used for the measurements. This sample geometry consists of separate modifications to measure the length of single-pulse modifications, which are surrounded by dense layers of modifications to allow the sample to be fractured. A laser scanning confocal microscopy (LSCM) micrograph of this sample type is shown in figure 6.3

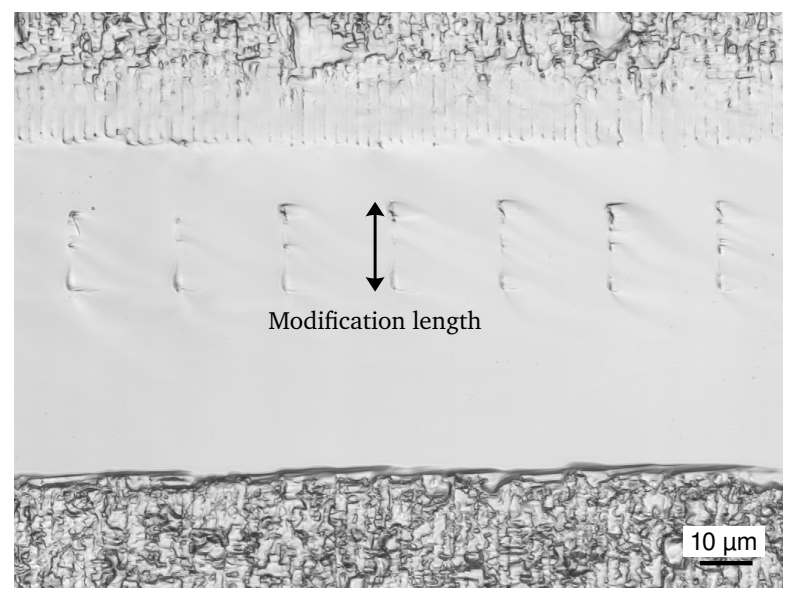

Figure 6.3: LSCM micrograph of a sample of geometry type (b). Two layers of dense modifications for sample separation (top and bottom) and a layer of single-pulse modifications for analysis (middle) are visible. Wavelength: $1549 \mathrm{~nm}$, pulse energy: $2 \mu \mathrm{J}$ (dense layers), $0.7 \mu \mathrm{J}$ (single-pulse modifications), transverse spacing: $2 \mu \mathrm{m}$ (dense layers), $20 \mu \mathrm{m}$ (single-pulse modifications). The laser beam propagation direction is from top to bottom.

The largest stochastic variations that were observed when measuring the length of different single-pulse modifications inside the same sample, or when comparing multiple samples obtained using the same processing conditions, were $\pm 2 \mu \mathrm{m}$. The most probable cause of these variations is the presence of small fluctuations in the laser pulse energy (see section 5.2.

\subsection{Parameter window for the formation of modifications}

To assess the suitability of different laser conditions for the formation of subsurface modifications in crystalline silicon, an experimental study has been performed using the total range of pulse durations that can be generated by the laser sources described in section 5.2. Only the presence of modifications was established by infrared microscopy, to 
find out which conditions should be selected for more detailed studies of the properties of the modifications. The results are summarised in table 6.2. For each laser source, it is indicated whether subsurface damage could be produced inside crystalline silicon.

Table 6.2: Suitability of lasers sources with various wavelengths and pulse durations for the formation of subsurface modifications in silicon, when combined with the optical system described in chapter 5 Depending on the processing conditions, different pulse energies are required. The pulse duration is defined as the full duration at half maximum (FDHM).

\begin{tabular}{lccl}
\hline Manufacturer and type & Wavelength & Pulse duration & Modifications found \\
\hline $\begin{array}{l}\text { Trumpf } \\
\text { Trumicro 5050 }\end{array}$ & $1030 \mathrm{~nm}$ & $6.6 \mathrm{ps}$ & No \\
\hline $\begin{array}{l}\text { Spectra Physics } \\
\text { Quanta Ray GCR-270 }\end{array}$ & $1064 \mathrm{~nm}$ & $8 \mathrm{~ns}$ & Yes \\
\hline $\begin{array}{l}\text { SPI } \\
\text { SP-020P-A-EP-S-A-Y }\end{array}$ & $1061 \mathrm{~nm}$ & $2-460 \mathrm{~ns}$ & Yes (all durations) \\
\hline $\begin{array}{l}\text { MWTechnologies } \\
\text { MOPA-LF-1550 }\end{array}$ & $1549 \mathrm{~nm}$ & & \\
\hline
\end{tabular}

Short nanosecond pulses combined with a wavelength of $1549 \mathrm{~nm}$ and both long and short nanosecond pulses with a wavelength around $1064 \mathrm{~nm}$ were found to be suitable for the formation of subsurface modifications. However, short picosecond pulses did not yield a positive result.

As the SPI laser source provides similar conditions as the Spectra Physics source while it has a better beam quality (see section 5.2 ), the modifications that were generated using the Spectra Physics source will be not further considered. However, it is interesting to note that a high beam quality is not a necessary condition for the formation of subsurface modifications in silicon.

When considering the results listed in table 6.2, the trends are consistent with the numerical data (see section 4.5.2). The suitability of short and long nanosecond pulses with a photon energy near the band gap, the suitability of short nanosecond pulses with a photon energy below the band gap and the unsuitability of picosecond pulses were all predicted by the numerical model.

Lower boundary of the pulse duration The shortest pulse duration of 2 ns that the $1061 \mathrm{~nm}$ source could emit was tested to check for evidence concerning the lower boundary of the pulse duration. For 2 ns pulses, modifications were only found for pulse energies between 0.14 and $0.91 \mu \mathrm{J}$, while changing the pulse energy in steps of $0.07 \mu \mathrm{J}$. Their visibility in infrared microscopy was poor at pulse energies of $0.7 \mu \mathrm{J}$ and above. For pulses with a duration of $8 \mathrm{~ns}$ and longer, clearly visible modifications were found for all pulse energies above the modifications threshold that were investigated (up to $4 \mu \mathrm{J}$ ). 
Consequently, the 2 ns pulse duration is expected to be close to the lower limit to the pulse duration. For a wavelength of $1064 \mathrm{~nm}$ and a perfect beam quality, the numerical prediction of this limit was approximately one nanosecond (see section 4.5.2.

The numerical simulations predicted that excessive two-photon absorption above the focus is an important factor causing the existence of a lower boundary of the pulse duration (see section 4.5.2). Supporting evidence for the correctness of this hypothesis is provided by the experiments with the $1549 \mathrm{~nm}$ laser source. This wavelength does not allow for linear interband absorption of laser energy, while the silicon is at room temperature. It was found that effective absorption of laser energy near the focus was possible at a pulse duration of $3.5 \mathrm{~ns}$ combined with a wavelength of $1549 \mathrm{~nm}$. As the equilibrium concentration of free carriers was too low to result in significant free carrier absorption (see section 6.2), the energy absorption is initially dominated by two-photon absorption. When reducing the pulse duration below $3.5 \mathrm{~ns}$, while keeping the pulse energy constant, effective two-photon absorption will also be possible further away from the focus.

The experimental data are consistent with the numerical prediction that self-focussing due to the Kerr effect is not a limiting factor for the parameter window of the pulse duration (see section 4.5.2). For $2 \mathrm{~ns}$ pulses at a wavelength of $1061 \mathrm{~nm}$, pulse energies of $0.98 \mu \mathrm{J}$ and above did not yield subsurface modifications. At this wavelength, equation (2.1) gives a critical power for self-focussing of $17 \mathrm{~kW}$. The corresponding critical energy, for laser pulses with a duration of $2 \mathrm{~ns}$ (FDHM) and a Gaussian temporal profile, is $36 \mu \mathrm{J}$. This value is well above the experimental limit of $0.98 \mu \mathrm{J}$. Consequently, other physical phenomena than the Kerr effect are determining the process window.

Suitability of picosecond pulses For 6.6 ps pulses combined with a wavelength of $1030 \mathrm{~nm}$, no conditions were found that were capable of producing any subsurface damage that could be observed by infrared microscopy. Pulse energies from $17.5 \mathrm{~nJ}$ to $2.1 \mu \mathrm{J}$ were tested. For a pulse energy of $2.1 \mu \mathrm{J}$, surface damage was observed, in spite of the fact that the focus of the beam was located $100 \mu \mathrm{m}$ below the sample surface. The following two questions may be raised regarding this experimental observation:

1. The wavelength $(1030 \mathrm{~nm})$ of the picosecond laser source is $31 \mathrm{~nm}$ lower than the nanosecond source with the shortest wavelength $(1061 \mathrm{~nm})$ that was found to be suitable (see table 6.2). In addition to the difference in pulse duration, the difference in wavelength may play a role, since the linear absorption coefficient is strongly wavelength-dependent for photon energies close to the band gap of silicon.

2. When using infrared microscopy, which has a limited resolution, there could be a risk of not detecting the presence of subsurface modifications.

To assess the influence of the difference in wavelength, some of the numerical simulations performed for $1064 \mathrm{~nm}$ have been repeated using a wavelength of $1030 \mathrm{~nm}$. The results of this comparison are shown in figure 6.4. While processing with the $1064 \mathrm{~nm}$ wavelength results in longer modifications according to the simulation results, the difference in wavelength alone is not expected to be the cause why no modifications could be obtained when processing with picosecond pulses. 


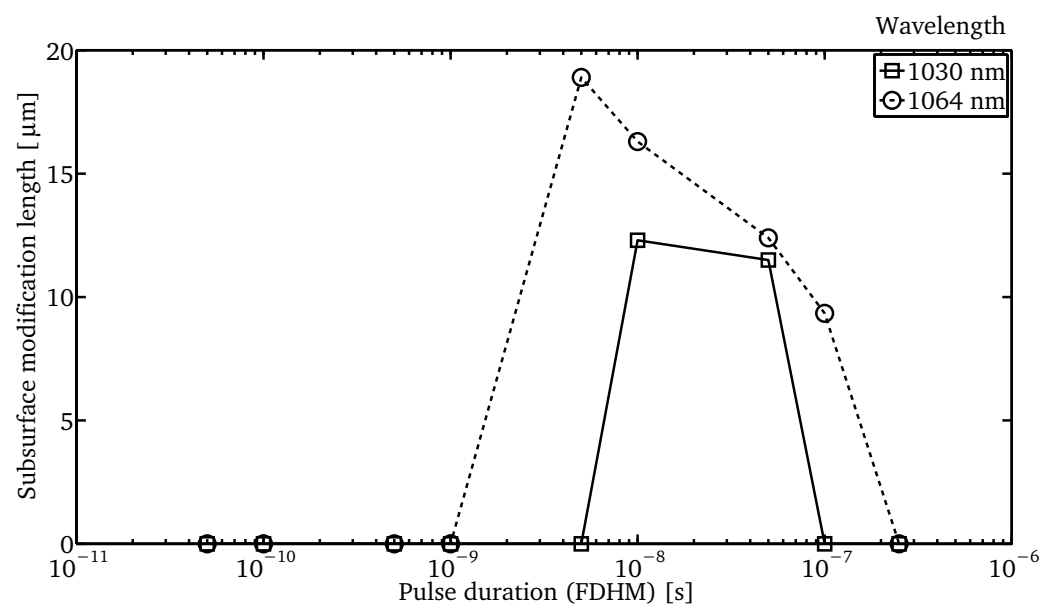

Figure 6.4: Subsurface modification length as a function of the pulse duration for two wavelengths. Pulse energy: $1 \mu \mathrm{J}$, focus depth: $100 \mu \mathrm{m}$.

Regarding question (2), infrared microscopy does not provide an absolute proof that no change to the material structure has occurred. To rule out the possibility of undetected damage to the best possible extent, the picosecond experiments have been repeated while applying many laser pulses to the same location. If undetectable subsurface damage would have been created by the first pulse, preferential laser energy absorption in the damaged volume is expected, causing additional damage during subsequent pulses. Even when applying twenty thousand pulses to the same location, no trace of subsurface damage could be found by infrared microscopy.

While excessive two-photon absorption above the focus was found to be the limiting factor regarding the use of ultra-short pulses, the critical pulse energy for self-focussing is in the order of a hundred nanojoules for the wavelength and pulse duration corresponding to the Trumpf laser source. Consequently, for most pulse energies that were tested, the Kerr effect is a contributing factor to the delocalization of the laser energy absorption when focussing picosecond pulses inside silicon.

In section 6.9 a number of possibilities to improve the usability of ultra-short pulses for the formation of subsurface modifications in crystalline silicon will be discussed.

\subsection{Model validation for subsurface modifications}

In addition to the validation of the numerical predictions regarding the feasibility of producing subsurface modifications, an explicit comparison between the experimental and numerical results concerning the geometry of the modifications was performed. In this section, the results of this comparison will be discussed for the $1061 \mathrm{~nm}$ and $1549 \mathrm{~nm}$ wavelengths. The presence of subsurface modifications and their geometry were measured according to the methods described in section 6.4 
$1061 \mathrm{~nm}$ wavelength The $1061 \mathrm{~nm}$ fibre laser can emit pulses with different temporal power profiles, by changing the profile of the seed pulses. Therefore, both the influence of the pulse duration and energy could be investigated.

Three different pulse durations were selected: 8,240 and 460 ns. The duration of $8 \mathrm{~ns}$ is slightly above the minimum achievable pulse duration of the laser source of $2 \mathrm{~ns}$. The $2 \mathrm{~ns}$ pulses were found to be unsuitable for the generation of modifications over a reasonable range of pulse energies (see section 6.5). The pulse duration of $460 \mathrm{~ns}$ represents the maximum pulse duration that can be emitted by the laser source. The $8 \mathrm{~ns}$ pulses correspond to a regime where both single- and two-photon absorption contribute to the generation of an electron-hole plasma in the bulk of silicon. For the 240 and $460 \mathrm{~ns}$ pulses, the intensities are too low to trigger two-photon absorption.

Comparisons between the experimental and simulated lengths of the modifications along the optical axis, as a function of the pulse energy, are shown in figures 6.5, 6.6 and 6.7 for the pulse durations of 8,240 and 460 ns respectively. The vertical error bars indicate the largest stochastic variations in the measurements of the modification lengths (see section 6.4). The horizontal error bars show the combined values of the uncertainty in the pulse energy measurements (see section 5.3 ) and the root mean square of the pulse energy fluctuations (see section 5.2 .

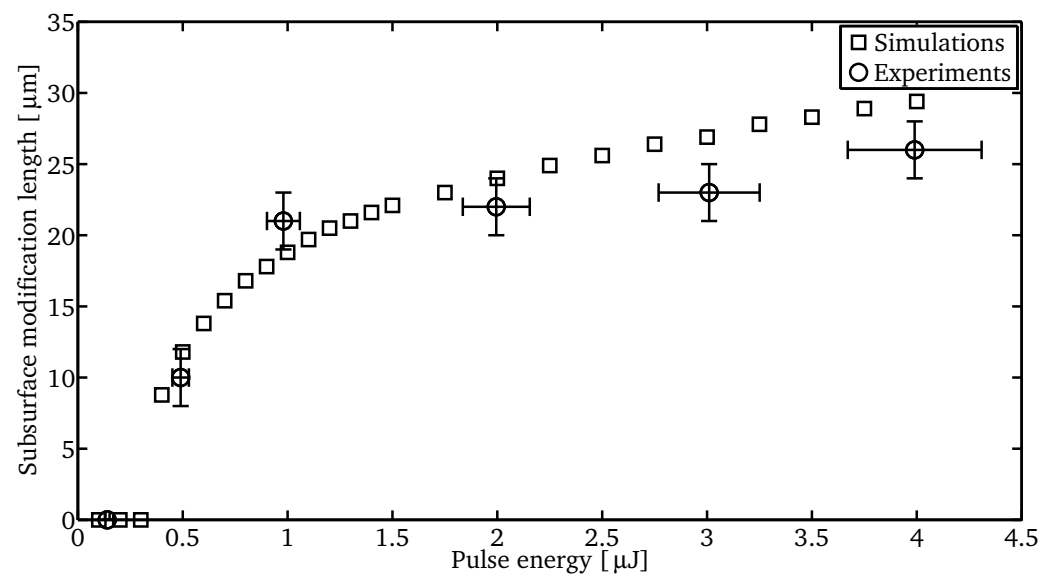

Figure 6.5: Comparison of experimental and simulated modification lengths along the optical axis. Wavelength: $1061 \mathrm{~nm}$, temporal pulse profile: Gaussian, pulse duration (FDHM): $8 \mathrm{ns,}$ $M^{2}=1.3$, focus depth: $100 \mu \mathrm{m}$. Spherical aberrations were compensated for during the production of the laser modifications.

The simulations and experiments show the same trend when increasing the pulse energy above the modification threshold. At first, the modification length quickly increases, while it saturates at higher pulse energies. Both the increasing diameter of the laser beam above the focus and plasma defocussing (see section 4.3.4) are responsible for this saturation behaviour. The modification threshold increases for longer pulse durations, as the lack of two-photon absorption and the diffusion of heat and free carriers result in less effective localised absorption of laser energy. 


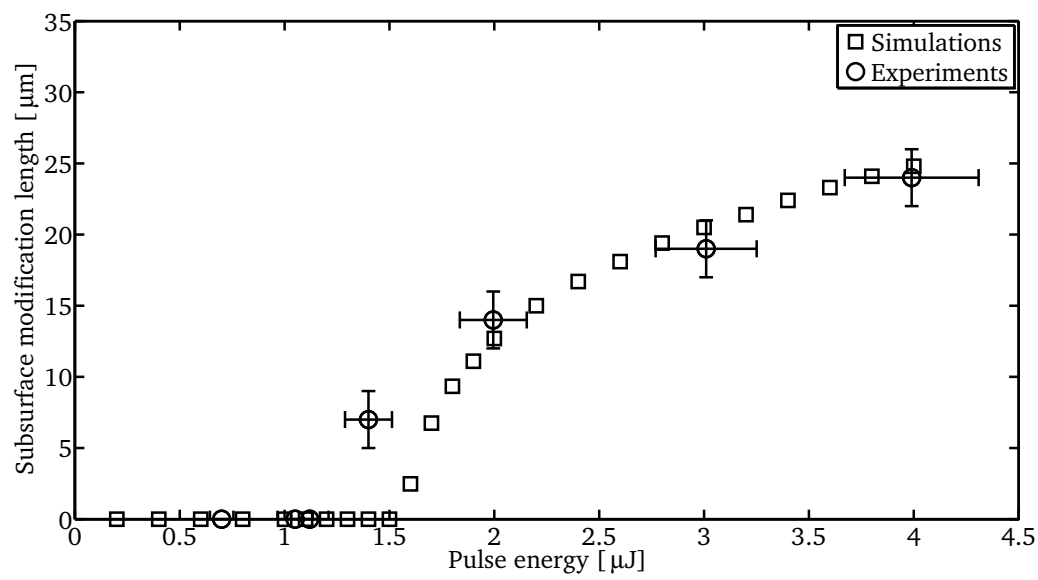

Figure 6.6: Comparison of experimental and simulated modification lengths along the optical axis. Wavelength: $1061 \mathrm{~nm}$, temporal pulse profile: constant power, pulse duration: $240 \mathrm{ns,}$ $M^{2}=1.3$, focus depth: $100 \mu \mathrm{m}$. Spherical aberrations were compensated for during the production of the laser modifications.

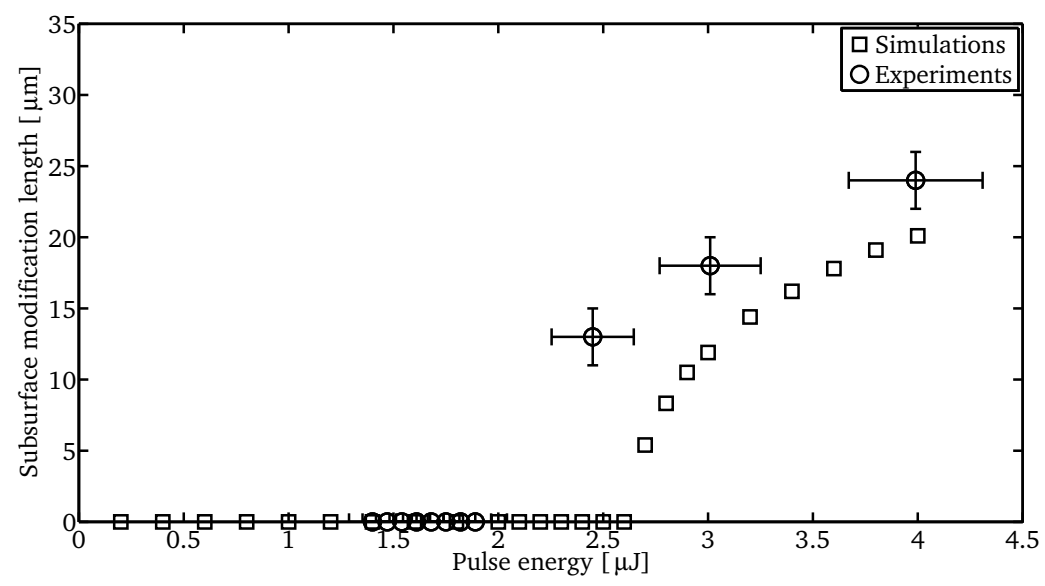

Figure 6.7: Comparison of experimental and simulated modification lengths along the optical axis. Wavelength: $1061 \mathrm{~nm}$, temporal pulse profile: constant power, pulse duration: $460 \mathrm{ns,}$ $M^{2}=1.3$, focus depth: $100 \mu \mathrm{m}$. Spherical aberrations were compensated for during the production of the laser modifications. 
$1549 \mathrm{~nm}$ wavelength In addition to the $1061 \mathrm{~nm}$ wavelength, experiments for the purpose of model validation have been performed with the $1549 \mathrm{~nm}$ laser. This laser source has a fixed pulse duration of $3.5 \mathrm{~ns}$. A comparison between the experimental and numerically computed lengths of the modifications can be found in figure 6.8 . Since data on the pulse energy stability was not available for the $1549 \mathrm{~nm}$ source (see section 5.2 ), it was assumed that the uncertainty in the peak power is representative for the uncertainty in the pulse energy.

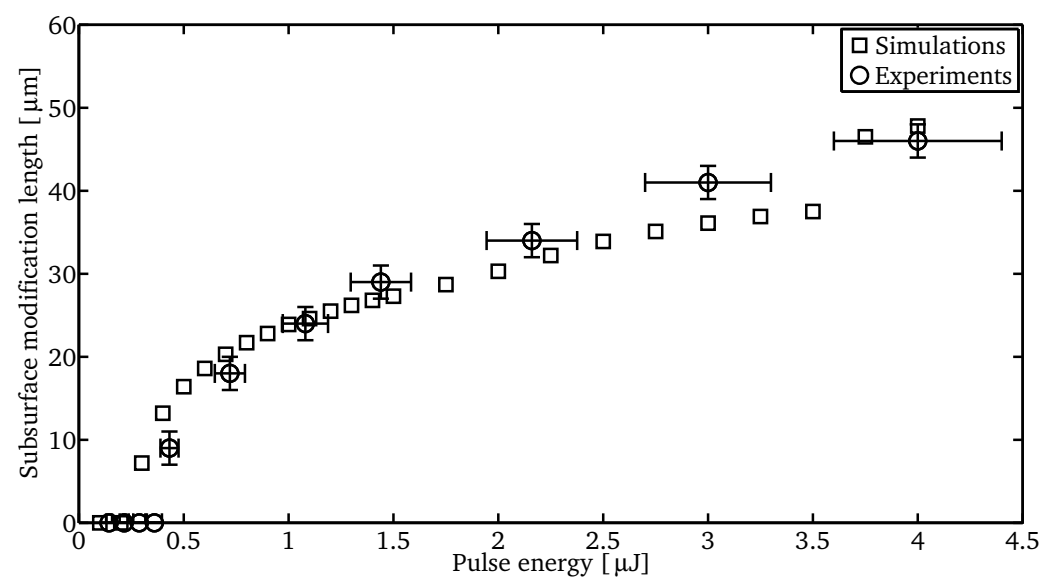

Figure 6.8: Comparison of experimental and simulated modification lengths along the optical axis. Wavelength: $1549 \mathrm{~nm}$, temporal pulse profile: Gaussian, pulse duration (FDHM): $3.5 \mathrm{ns,}$ $M^{2}=1.1$, focus depth: $100 \mu \mathrm{m}$. Spherical aberrations were compensated for during the production of the laser modifications.

The $1549 \mathrm{~nm}$ wavelength resulted in the generation of significantly longer modifications compared with the $1061 \mathrm{~nm}$ process. While this is partly caused by the slower decay of the laser intensity along the optical axis due to the longer wavelength, an important factor is that the generation of free carriers at undesired locations has been prevented. Due to the linear interband absorption that occurs at $1061 \mathrm{~nm}$, additional free carriers are generated throughout the beam path inside silicon, proportional to the total energy that passes through this trajectory. If the concentration of free carries along the path of the laser beam becomes too high, any further energy that is emitted by the laser source will not effectively contribute to growth of the subsurface modifications due to plasma defocussing (see section 4.3.4).

Accuracy of the numerical predictions When comparing the experimental and numerical results in figures $6.5,6.8$, a reasonable match can be observed. The majority of the numerical predictions of the modification length are within $5 \mu \mathrm{m}$ of the experimental data. The main factors that are expected to be responsible for the observed deviations between the predicted and measured modification lengths are listed below.

- The imperfect description of the physical reality by the equations employed in the numerical model. Especially the assumptions that were made during the derivation 
of the beam propagation model from Maxwell's equations (see section 3.3.2) will result in errors.

- Inaccuracies in the material properties that were implemented in the numerical model (see section 3.5). These are in particular caused by the extrapolation of material data towards temperatures and carrier densities beyond the range of conditions that were measured.

Due to the large number of equations and material properties that affect the numerical results shown in this section, limited differences between experimental and numerical results cannot be attributed to a specific cause. However, one discrepancy can be related to sources of errors with reasonable certainty. Significantly larger deviations are visible for 460 ns pulses compared with shorter pulse durations. The numerical predictions overestimate the threshold energy and consequently underestimate the modification lengths. Due to the dependence on the pulse duration, this discrepancy is likely to be caused by the accuracy of material data that are related to physical phenomena that are important for the modelling of long pulses. These phenomena are the conduction of heat and free carriers and the recombination of electron-hole pairs (see section 2.5).

Since some deviations between the simulated and measured modification shapes were observed, it is recommended to select processing conditions that are not too close to the predicted process boundaries. This ensures that the selected conditions do not fall outside the actual process window due to inaccuracies in the numerical results.

\subsection{Wafer dicing}

In this section, the suitability of the subsurface modifications for wafer dicing will be considered. For this purpose, samples of geometry type (a) (see section 5.5) were produced using various processing parameters. After laser processing, the samples were slightly bent to induce stresses, which should cause the samples to fracture along the modifications. It was found to be possible to create fracture planes that follow the intended planes throughout the entire thickness of the wafer, as required for wafer dicing (see figure 6.9). Successful dicing was achieved using both the 1061 and $1549 \mathrm{~nm}$ processes.

In section 1.3.2 an overview of the criteria to assess the quality of the dicing process has been given. During the experimental study, the kerf geometry and damage to the side walls were considered. The die strength was not measured. Three out of four edges of the dies that were created by subsurface separation were pre-diced. When measuring the strength of the dies, it is generally unknown which side wall induced the catastrophic failure of the die. Therefore, for die strength measurements, all edges should be diced using the same technology.

While quantitative measurements were not performed, it was found that the application of pulses of several hundred nanoseconds resulted in lower separation forces compared with short nanosecond pulses. This was deduced from the amount of elastic deformation of the samples before they fractured. Long nanosecond pulses would sometimes induce die separation during the laser process, before a force was exerted on the sample. This behaviour was not observed for shorter pulses. 


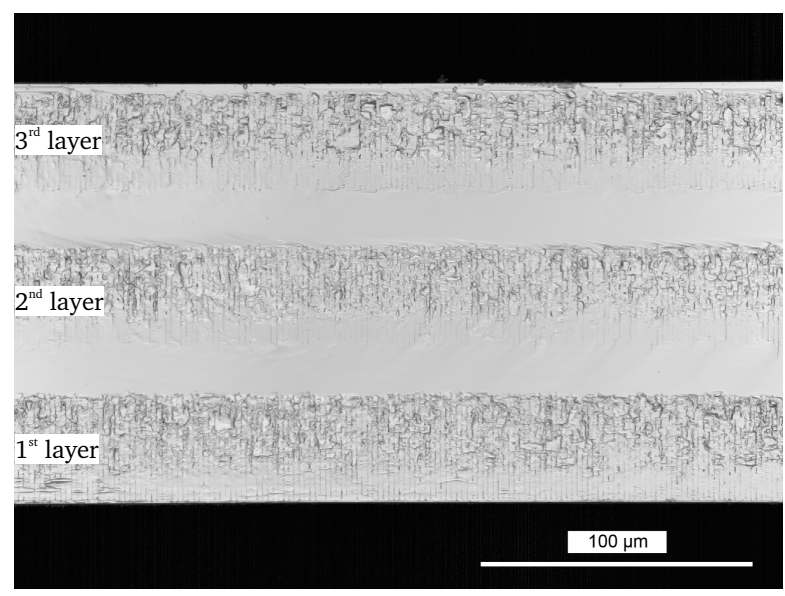

Figure 6.9: LSCM micrograph of a fracture plane obtained after dicing a 160- $\mu \mathrm{m}$ thick silicon wafer. Wavelength: $1549 \mathrm{~nm}$, pulse energy: $1.3 \mu \mathrm{J}$, transverse spacing modifications: $2 \mu \mathrm{m}$. Three different focus depths inside the wafer were employed. The laser beam propagation direction is from top to bottom.

\section{Kerf geometry}

It was found that a transverse spacing between the subsurface modifications below $2-3 \mu \mathrm{m}$ is a necessary condition for the fracture plane to coincide with the modifications. For a spacing of $4 \mu \mathrm{m}$, the samples broke around the intended dicing streets, but the fracture planes were not aligned with the modifications. When placing modifications with a transverse spacing of $2 \mu \mathrm{m}$ near the front and back surface of the wafer, a reasonable kerf geometry was often observed (see figure 6.10). However, deviations of the kerf geometry from a flat plane were occasionally found between modified layers, as shown in figure 6.11

Issues concerning the kerf geometry were mostly encountered near the front or back surface of the wafer. An example can be found near the bottom surface of the die shown in figure 6.11. The reason why this die was affected by kerf geometry deviations near the surface, is because the modifications were located at larger distances from the wafer surface compared with the result presented in figure 6.9. If the distances between both surfaces and the nearest subsurface modifications were unequal, the best kerf geometry was obtained when inducing tensile stresses near the surface that is closest to the modifications.

As is shown in figure 6.11 issues with the kerf geometry are not limited to the vicinity of the surface. A trench is visible at a distance of tens of micrometres from the back surface of the wafer. It was found that the formation of such trenches can be prevented by modifying a large part of the intended fracture plane. For a fixed wafer thickness, this can be accomplished by fewer modified layers consisting of tall modifications, or by a larger number of layers build-up of smaller modifications. Tall modifications can be produced when selecting a pulse energy well above the modification threshold. Examples of both approaches are shown in figures 6.12 and 6.13 . No appreciable differences have been found between these approaches. Consequently, the use of a limited number of modified layers containing tall modifications is recommended from a process efficiency point of view. The 


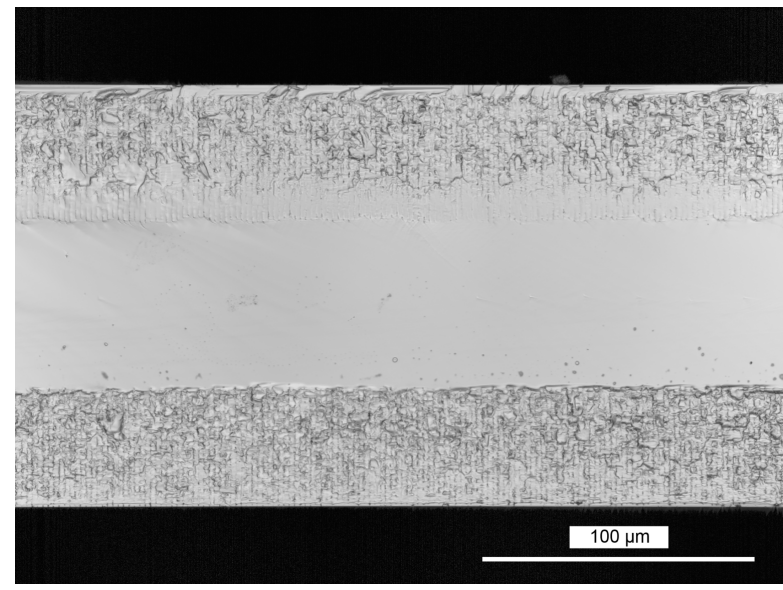

Figure 6.10: LSCM micrograph of a fracture plane obtained after dicing a 160- $\mu \mathrm{m}$ thick silicon wafer. Wavelength: $1549 \mathrm{~nm}$, pulse energy: $2 \mu \mathrm{J}$, transverse spacing modifications: $2 \mu \mathrm{m}$. Two different focus depths inside the wafer were employed. The laser beam propagation direction is from top to bottom.

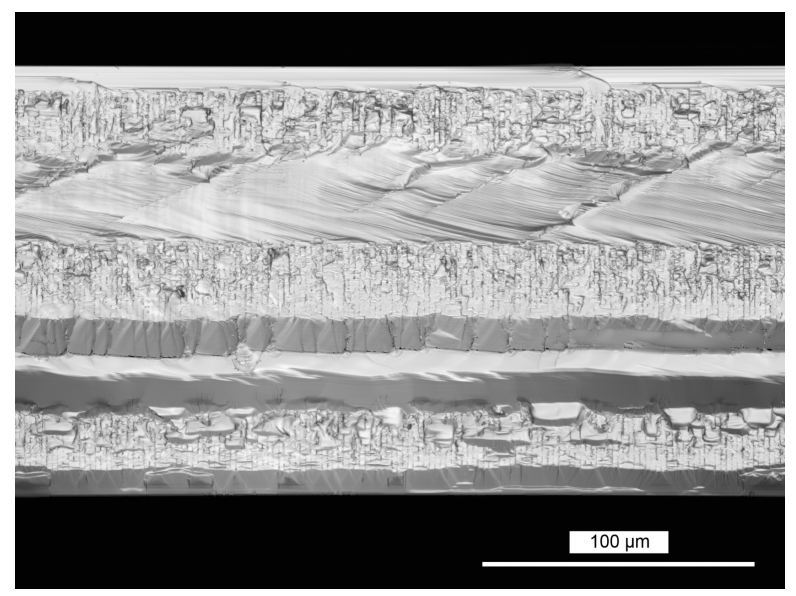

Figure 6.11: LSCM micrograph of a fracture plane obtained after dicing a $160-\mu \mathrm{m}$ thick silicon wafer. Wavelength: $1549 \mathrm{~nm}$, pulse energy: $1.3 \mu \mathrm{J}$, transverse spacing modifications: $2 \mu \mathrm{m}$. Three different focus depths inside the wafer were employed. The laser beam propagation direction is from top to bottom. Deviations from the intended kerf geometry between modified layers and at the bottom of the wafer are visible. 


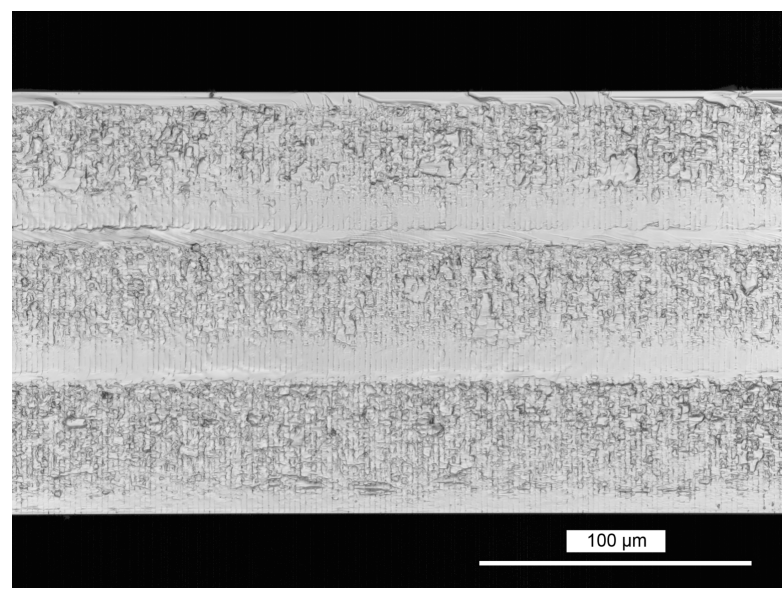

Figure 6.12: LSCM micrograph of a fracture plane obtained after dicing a 160- $\mu \mathrm{m}$ thick silicon wafer. Wavelength: $1549 \mathrm{~nm}$, pulse energy: $2 \mu \mathrm{J}$, transverse spacing modifications: $2 \mu \mathrm{m}$. Three different focus depths inside the wafer were employed. The laser beam propagation direction is from top to bottom.

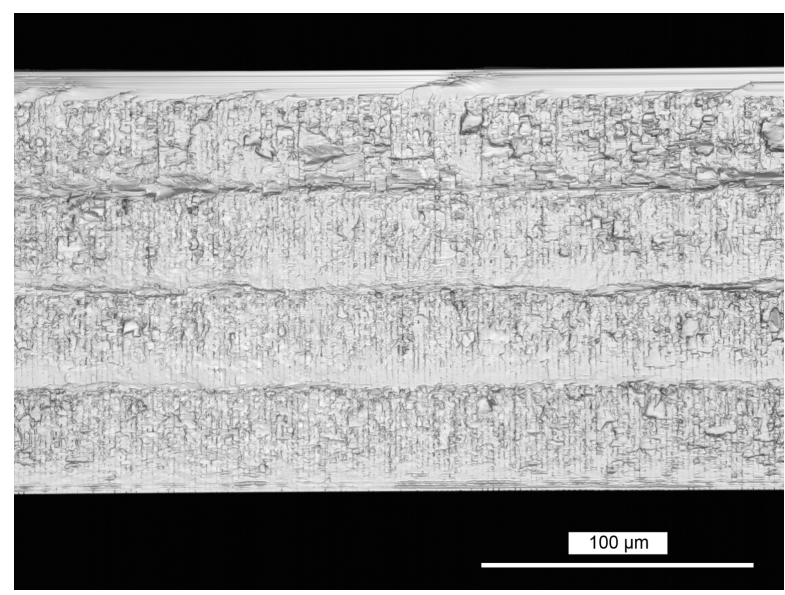

Figure 6.13: LSCM micrograph of a fracture plane obtained after dicing a $160-\mu \mathrm{m}$ thick silicon wafer. Wavelength: $1549 \mathrm{~nm}$, pulse energy: $1.3 \mu \mathrm{J}$, transverse spacing modifications: $2 \mu \mathrm{m}$. Four different focus depths inside the wafer were employed. The laser beam propagation direction is from top to bottom. 
required pulse energy to form a sufficiently tall layer of modifications may deviate from measurements of the geometry of single-pulse modifications. This is due to the fact that closely spaced laser pulses interact with previously modified material.

It was found that deviations from the intended kerf geometry are caused by the fracture plane switching to a preferential cleavage plane of the silicon crystal. The angles of the side walls of the trench shown in figure 6.11, were measured using laser scanning confocal microscopy. The preferential \{111\} cleavage planes (see section 2.7) of diamond cubic silicon intersect the surface of a $<100>$ wafer at an angle of $\operatorname{atan}(\sqrt{2}) \approx 55^{\circ}$ (see figure 2.1). The measured angles were close to this value. Due to the influence of preferential cleavage planes, the orientation of the dicing streets with respect to the silicon crystal will affect the dicing process.

\section{Side wall damage}

The surface profile of the side wall of the die presented in figure 6.9 was measured by confocal microscopy, along lines perpendicular to the optical axis. The worst-case deviations from a flat surface were found to be approximately $\pm_{1} \mu \mathrm{m}$. The $R_{a}$ roughness of the modified layers was between 150 and $200 \mathrm{~nm}$, while the roughness of the unmodified material was smaller than $100 \mathrm{~nm}$.

Wafer separation mechanisms The failure mode of Si-I at room temperature is brittle failure by cleavage along specific crystallographic planes (see section 2.6). When performing dicing tests, the laser-modified samples were also found to break in a brittle manner.

Evidence of crack growth, starting at the laser-induced modifications, has been found on fracture planes, as shown in figure 6.14. The initiation of cracks was observed for both separate and overlapping laser modifications. Cracks were also detected by infrared microscopy, before separating the wafer (see section 6.8). These subsurface cracks serve as initiation points for further crack growth (see section 2.7).

Because it was found that modifying a large part of the fracture plane results in the best kerf geometry, the modifications are likely to have a second function beyond the initiation of cracks. A hypothesis is that the cracks follow boundaries between different material phases.

\subsection{Modification mechanisms}

In this section, the modification mechanisms during laser processing are discussed, based on the information that can be extracted from fracture planes. In section 7.7 , this discussion will be continued based on analyses of the crystal structure of the subsurface modifications.

\section{Melting and resolidification}

The modifications shown in figure 6.3 have a line shape and do not have a larger width further away from the focus. Similar lines were also observed when analysing closely spaced modifications. A scanning electron microscopy (SEM) micrograph of the second modified layer in figure 6.9 is shown in figure 6.15. Vertical lines are visible, with a spacing of $2 \mu \mathrm{m}$ that corresponds to the transverse spacing between the laser pulses. 


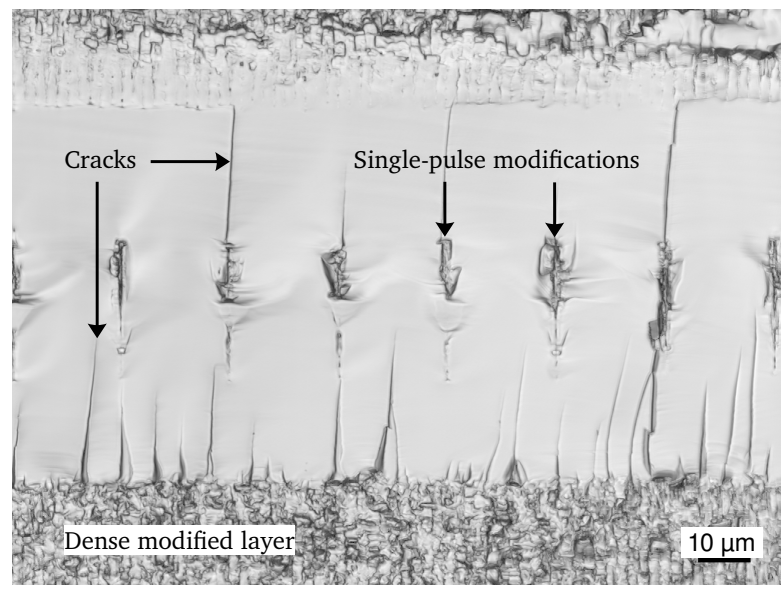

Figure 6.14: LSCM micrograph of a fracture plane obtained after dicing a 160- $\mu \mathrm{m}$ thick silicon wafer. Spherical aberrations were compensated for during the production of the single-pulse modifications. Wavelength: $1549 \mathrm{~nm}$, pulse energy: $1.1 \mu \mathrm{J}$. The laser beam propagation direction is from top to bottom. The propagation of cracks from the laser modifications is visible.

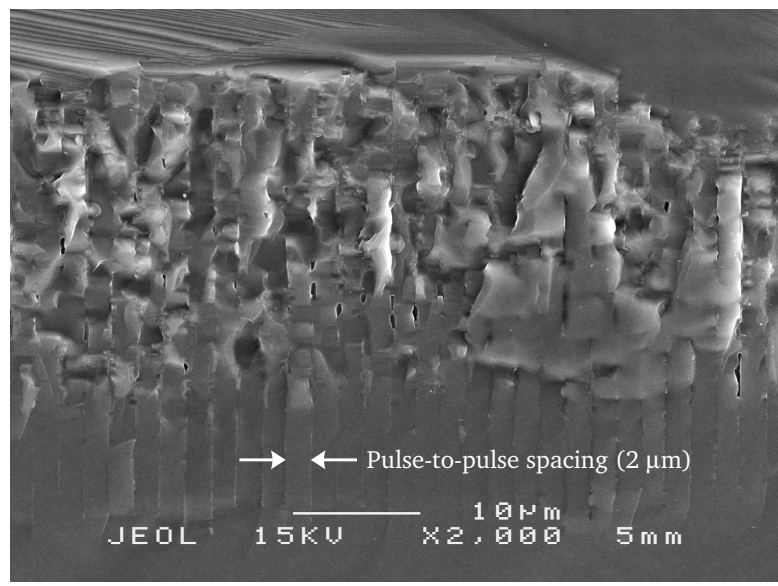

Figure 6.15: SEM micrograph (secondary electrons) of a fracture plane obtained after dicing a $160-\mu \mathrm{m}$ thick silicon wafer. Wavelength: $1549 \mathrm{~nm}$, pulse energy: $1.3 \mu \mathrm{J}$, transverse spacing modifications: $2 \mu \mathrm{m}$. The second layer indicated in figure 6.9 is shown. The laser beam propagation direction is from top to bottom. 
The line-shapes do not match the beam profile inside the sample, as the beam diverges above the focus. As the modification process starts in the focus of the beam, it is reasonable to assume that the line-shaped modifications are located on the optical axis. A hypothesis regarding this shape is that it is related to the location where the silicon resolidifies last.

\section{Formation of voids}

A possible secondary modification mechanism after a volume of silicon has reached the vapour phase is the formation of voids. Randomly distributed voids were found on fracture planes containing closely spaced modifications. A detail of the modifications that are presented in figure 6.15 is shown in figure 6.16. Several voids can be observed.

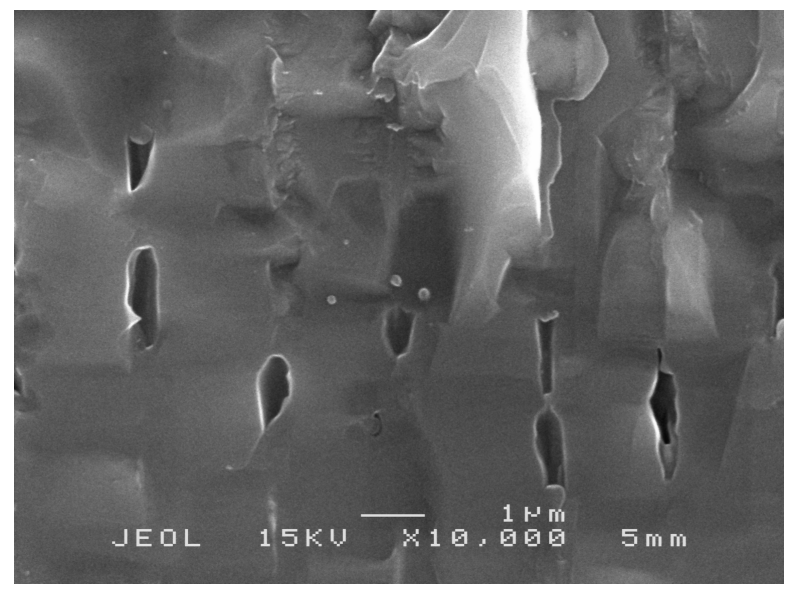

Figure 6.16: SEM micrograph (secondary electrons) of a fracture plane obtained after dicing a $160-\mu \mathrm{m}$ thick silicon wafer. Wavelength: $1549 \mathrm{~nm}$, pulse energy: $1.3 \mu \mathrm{J}$, transverse spacing modifications: $2 \mu \mathrm{m}$. A detail of the modifications presented in figure 6.15 is shown. The laser beam propagation direction is from top to bottom.

Perpendicular to the optical axis, the dimensions of the voids are in the order of 100$500 \mathrm{~nm}$. This small size can explain the inability to detect them using a CT-scan (see section 5.6. Since a void should provide a strong difference in X-ray absorption compared with the surrounding material, any void that would be present has to be smaller than the detectable feature size. The voxel size of the cT-scanner that was employed is $500 \mathrm{~nm}$.

A disadvantage of the analysis of voids after fracturing is that it does not constitute a proof that the voids are a direct result of the laser-material interaction. Possible explanations for the formation of voids can be found in section $7 \cdot 7$.

\section{Formation of cracks}

Evidence of the formation of cracks during laser processing with long nanosecond pulses was found by infrared transmission microscopy. A comparison between 8 and $460 \mathrm{~ns}$ pulses with a wavelength of $1061 \mathrm{~nm}$ and the same pulse energy is shown in figures $6.17 \mathrm{a}$ and $6.17 \mathrm{~b}$. While no cracks are visible for the $8 \mathrm{~ns}$ pulse duration, the formation of cracks that sometimes interconnect the modifications can be observed for the $460 \mathrm{~ns}$ pulses. An 


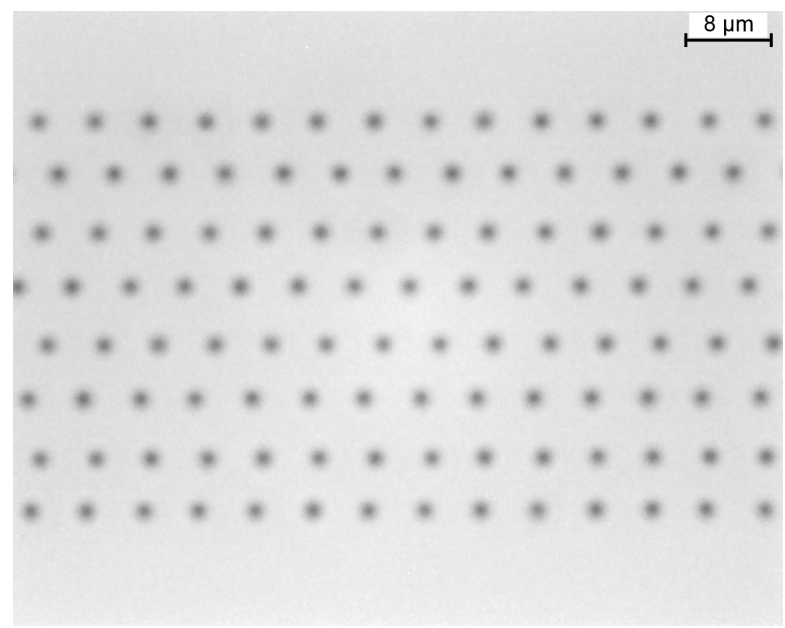

(a) Pulse duration (FDHM): 8 ns.

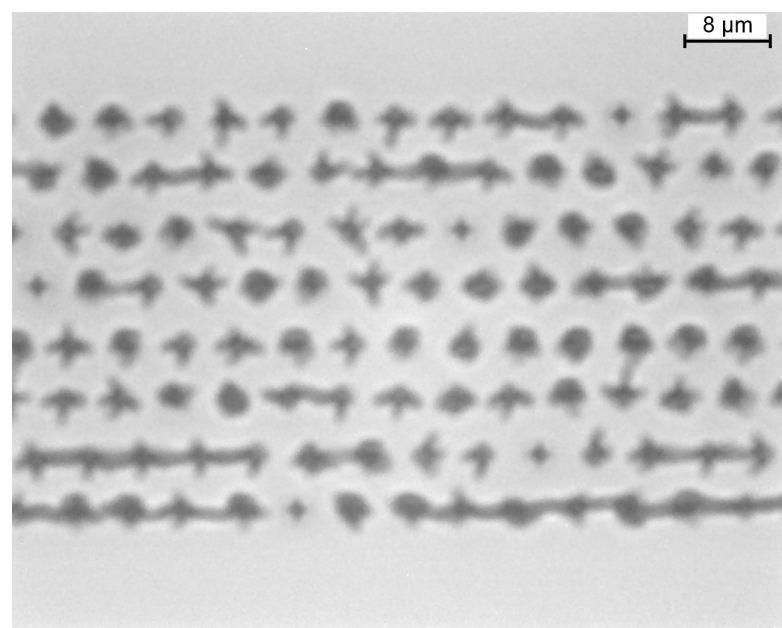

(b) Pulse duration (FDHM): 460 ns.

Figure 6.17: Infrared transmission micrographs of subsurface modifications inside silicon (top view). Eight horizontal laser tracks were produced containing modifications spaced $5 \mu \mathrm{m}$ apart, while compensating for spherical aberrations. Pulse energy: $2.8 \mu \mathrm{J}$, wavelength: $1061 \mathrm{~nm}$, focus depth: $100 \mu \mathrm{m}$. 
effect of the pulse duration has also been found during subsurface processing of fused silica, when comparing pico- and femtosecond pulses [17].

Modifications obtained using long nanosecond pulses were found to result in a lower separation force compared with short nanosecond pulses (see section 6.7). It is plausible that the cracks shown in figure $6.17 \mathrm{~b}$ are responsible for the decrease in the separation force. However, some of these cracks will also be present near the edges of separated dies, which is likely to have a negative impact on the die strength.

Numerical simulations showed that sufficiently high stresses to induce failure of silicon are unlikely to occur before the onset of subsurface melting (see section 4.4). Therefore, the crack growth presumably took place after an initial defect was created by thermal melting, either during the laser pulse, during the cooling phase after the pulse or due to thermal stresses imposed by the formation of neighbouring modifications.

\subsection{Improving the usability of ultra-short pulses}

Subsurface modifications could not be created using 6.6 ps pulses, while nanosecond pulses were successfully applied (see section 6.5). In this section, possible changes to the experimental conditions to improve the usability of ultra-short pico- to femtosecond pulses are discussed. Whether such pulses have advantages for the fabrication of modifications inside silicon is presently unknown. It is possible that shortening the pulse duration results in different material structures of the subsurface modifications compared with a nanosecond process. Moreover, it is likely that non-thermal melting (see section 2.6) instead of thermal melting will occur. However, to be able to create subsurface modifications with sub-nanosecond pulses, the problem of delocalization of the laser energy absorption (see section 4.5.2. has to be addressed.

\section{Higher-order nonlinear absorption}

A solution could be to employ higher-order nonlinear absorption to improve the spatial confinement of the laser energy absorption. The lattice absorption in silicon is limited until a wavelength of $6 \mu \mathrm{m}$ [94]. Therefore, the use of longer wavelengths is possible. This would result in an increase in the intensity at which efficient absorption of laser energy occurs. Disadvantage of the use of longer wavelengths is that the size of the focal spot increases. Moreover, up to a wavelength of $6 \mu \mathrm{m}$, the Kerr coefficient is either similar to or above its value at the wavelengths that were considered in this work [90]. Consequently, it is important to ensure that the required instantaneous power to obtain sufficiently high intensities for multiphoton absorption does not exceed the critical power for self-focussing (see section 2.3). Additionally, the availability of laser sources will have to be considered. The development of high efficiency fibre lasers for mid-infrared wavelengths has not been as successful as for near-infrared radiation [171], although significant progress is still being made [172].

\section{Increasing the numerical aperture}

Apart from exploiting higher-order nonlinear processes, employing an objective with a higher numerical aperture than 0.7 would improve the usability of short pulses, by inducing a faster decay of the laser intensity above the focus. Consequently, inadvertent multiphoton 
absorption of laser energy outside the vicinity of the focal plane can be reduced. However, previous research has shown that only using an oil immersion objective with a numerical aperture greater than one, is insufficient to prevent surface damage during a femtosecond process [64]. Moreover, there are practical issues associated with objectives which have a very high numerical aperture. In general, higher numerical aperture objectives have a shorter working distance, preventing the focus from being placed deep inside the material. Additionally, numerical apertures greater than one are only possible when using an immersion fluid, which partly negates the cleanliness of the subsurface dicing process. Furthermore, higher numerical apertures require a larger area on the surface of the wafer to be free of structures, to prevent distortion of the laser beam as it propagates into the wafer. Consequently, wider dicing streets are needed.

\section{Temporal focussing}

Finally, a third option exists to enhance the suitability of ultra-short pulses, which is temporal confinement [173, 174]. This method is based on the fact that a femtosecond pulse will by definition have a relatively large bandwidth, as the amplitude of the optical wave is modulated on a short time scale. This relation between the pulse duration and bandwidth directly follows from the Fourier transform. When the different wavelengths are separated in space, the pulse duration will increase. By recombining the wavelengths in the geometrical focus of the beam, the pulse duration in the focus is shortened compared with its surroundings.

\subsection{Summary}

In this chapter, experimental results regarding the geometry of laser-induced subsurface modifications were presented, including an assessment of their suitability for the subsurface dicing process. Subsurface modifications were successfully created using the following combinations of wavelengths and pulse durations: $1061 \mathrm{~nm} / 2-460 \mathrm{~ns}, 1064 \mathrm{~nm} / 8 \mathrm{~ns}$ and $1549 \mathrm{~nm} / 3.5 \mathrm{~ns}$.

All modifications that were produced were found to be suitable for the laser-induced subsurface separation method. The geometry and positioning of the modifications had a strong influence on the quality of the dicing process. Observed quality issues were deviations from the desirable kerf geometry and rough fracture planes. To prevent issues with the kerf geometry, locating the modifications close to both surfaces of the wafers and modifying a large part of the intended fracture plane were found to be useful strategies. The $1549 \mathrm{~nm}$ process was found to be better suited for the efficient production of tall modifications. The $1061 \mathrm{~nm}$ process allows for long nanosecond pulses to be employed, which yield a lower separation force compared with short nanosecond pulses, presumably due to the formation of subsurface cracks. However, some of these cracks will also remain in the finished product and potentially have a negative influence on the die strength.

The functions of the subsurface modifications during the dicing process are likely to be the initiation of cracks and the guidance of their propagation. Since quality issues were often a result of the fracture plane switching to a preferential cleavage plane of the silicon crystal, the orientation of the dicing streets with respect to the crystal is of importance. 
The $1549 \mathrm{~nm}$ process relies on two-photon absorption to generate an electron-hole plasma. Therefore, the objective of developing a multiphoton process, which is suitable to create subsurface modifications near the focus of the laser beam, has been achieved. The research concerning the formation of subsurface modifications using 6.6 ps pulses had a negative outcome. As only a narrow range of pulse energies resulted in the formation of modifications for the combination of a $1061 \mathrm{~nm}$ wavelength with a $2 \mathrm{~ns}$ pulse duration, this duration is expected to be close to the lower boundary of the process window for this wavelength.

The experimental results were compared with data from numerical simulations. A reasonable match between the experimental and simulated modification thresholds and shapes was obtained. Moreover, the numerical model correctly predicted which laser sources were suitable for the formation of subsurface modifications. Consequently, both the primary and secondary goals (see section 3.1) of the numerical model have been fulfilled. 


\section{Experimental results: crystal structure}

In this chapter, ${ }^{*}$ the analysis of the crystal structure of modifications that were produced using the set-up described in chapter 5 will be presented. Based on this analysis, the mechanisms that contributed to the formation of subsurface modifications are identified.

\subsection{Introduction}

In chapter 6, the geometry of laser-induced subsurface modifications has been evaluated. In this chapter, the crystal structure of the modifications will be considered. Lamellae containing cross-sections of modifications were prepared for analysis by transmission electron microscopy. In addition to brightfield micrographs, selected area diffraction patterns were recorded to investigate the crystal structure of specific parts of the subsurface modifications. Moreover, Raman spectroscopy was employed. Special attention was given to the detection of high pressure phases, as the generation of significant pressures is known to occur when focussing laser pulses inside dielectrics (see section 2.6).

The purpose of the analysis of the crystal structure is twofold. First, the analysis results are used to identify the material modification mechanisms that occur during the lasermaterial interaction. Consequently, the mechanisms that were included in the numerical model can be validated. Secondly, the obtained overview of the different crystal structures that are created by the laser process, can be employed to assess the feasibility of applications of laser-induced modifications in bulk silicon.

The modifications that are analysed in this chapter were created inside the same base material as described in section 6.2, using the $1549 \mathrm{~nm}$ laser source with a pulse duration of $3.5 \mathrm{~ns}$ (see section 5.2). A 250- $\mu \mathrm{m}$ thick quartz window was located behind the microscope objective during the formation of the modifications. Consequently, spherical aberrations were present that affect the shape of the laser modifications (see section 5.4.1).

\subsection{Lamella preparation}

As indicated in section 5.7, two methods to produce electron-transparent lamellae for transmission electron microscopy (TEM) have been applied. These methods result in cross-sections along and perpendicular to the optical axis (see figure 7.1). In this section, micrographs obtained during the preparation of cross-sections with both orientations are presented.

\footnotetext{
${ }^{*}$ The transmission electron microscopy analysis of cross-sections along the optical axis has been performed at NXP Semiconductors, Nijmegen, The Netherlands. The analysis of cross-sections perpendicular to the optical axis and the Raman Spectroscopy were performed by L. Smillie of The Australian National University, Canberra, Australia.
} 


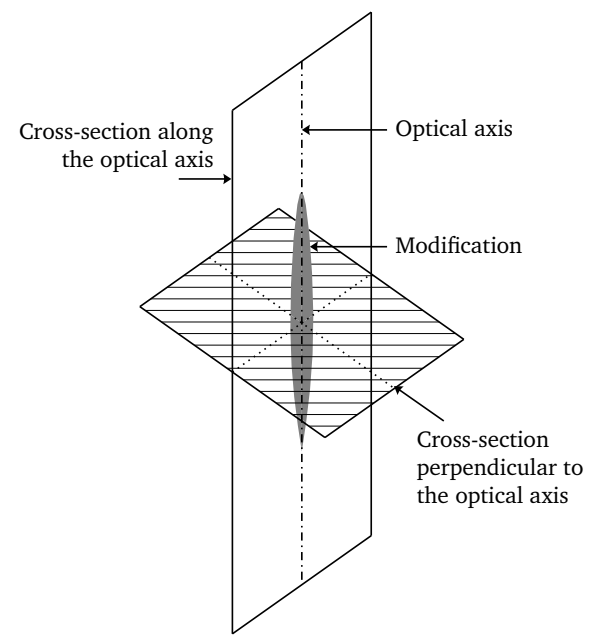

Figure 7.1: Drawing of the orientations of the TEM lamellae with respect to the modifications and the optical axis.

\subsubsection{Cross-sections along the optical axis}

In order to produce cross-sections along the optical axis (see figure 7.1), tracks of subsurface modifications were created adjacent to surface marks (see sample geometry type (c) in section 5.5). These marks allow the subsurface modifications to be located. A scanning electron microscopy (SEM) micrograph of this sample type, before the production of the lamella, is shown in figure 7.2 .

As the amount of material that can be removed by focussed ion beam (FIB) milling is limited, the modifications had to be positioned just below the surface. To achieve this goal, the distance between the sample and the objective was increased by $500 \mathrm{~nm}$ per track, until the modifications were visible on the surface (see figure 7.2). The deepest modifications were created first, while the surface marks were produced last. Consequently, surface damage was only induced after the production of subsurface modifications was finished. Surface damage or debris has a negative influence on the propagation of the laser beam into the sample. A pulse energy of $0.6 \mu \mathrm{J}$ was selected, which is close to the modification threshold, to create short modifications that fit inside the lamellae.

The tracks of modifications that were closest to the surface, without showing surface damage, were used to expose modifications by FIB milling. Platinum was deposited on the wafer surface at the locations where lamellae were produced, to prevent surface damage during the ion beam milling. The lamellae were "plucked-out" in-situ.

SEM micrographs of the lamellae were recorded during thinning. Two of these micrographs are shown in figures $7.3 \mathrm{a}$ and $7.3 \mathrm{~b}$, corresponding to different stages of the thinning process. Cracks are visible at the locations where modifications are present. While the cracks are initially located below the surface (see figure $7 \cdot 3 \mathrm{a}$ ), they grow towards the wafer surface during thinning (see figure 7.3b). Therefore, the lamellae were kept relatively thick to prevent structural failure. 


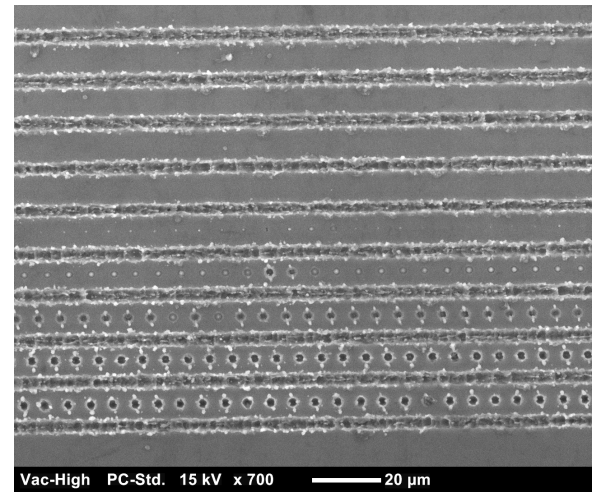

Figure 7.2: SEM micrograph of the surface of a sample of geometry type (c), intended for further processing by FIB. Nine tracks of separate modifications were created between surface marks. From top to bottom, the sample was moved $500 \mathrm{~nm}$ further away from the focussing objective per track. Wavelength: $1549 \mathrm{~nm}$, pulse energy: $0.6 \mu \mathrm{J}$, transverse spacing modifications: $5 \mu \mathrm{m}$.

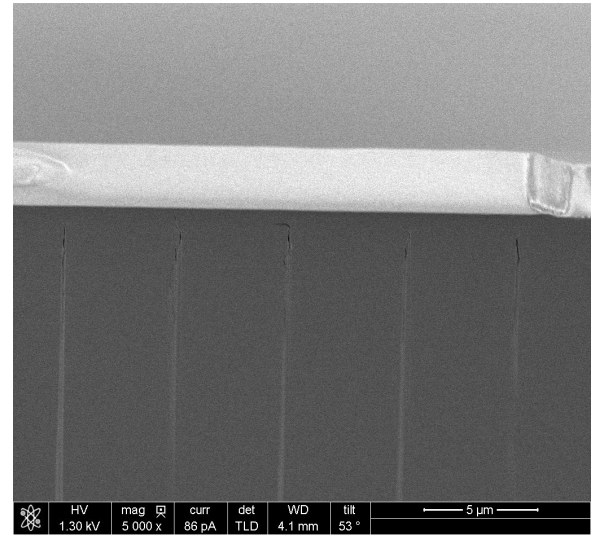

(a) Lamella with a thickness of several micrometres.

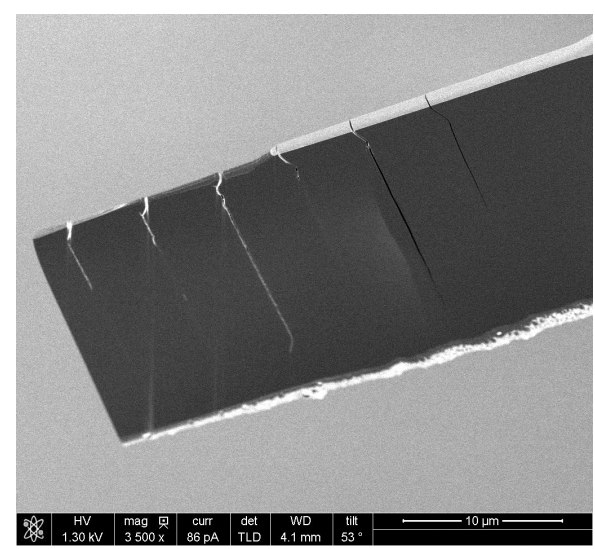

(b) Electron-transparent lamella.

Figure 7.3: SEM micrographs of the production of a TEM lamella using a FIB. The laser modifications were located just below the surface and are spaced $5 \mu \mathrm{m}$ apart. Wavelength: $1549 \mathrm{~nm}$, pulse energy: $0.6 \mu \mathrm{J}$. The propagation direction of the laser beam is from top to bottom. 


\subsubsection{Cross-sections perpendicular to the optical axis}

To create lamellae containing cross-sections of subsurface modifications perpendicular to the optical axis (see figure 7.1), a combination of mechanical polishing and FIB milling was employed. Sample geometry type (d) (see section 5.5) was used for this purpose. The polishing procedure was started at a fracture plane that was parallel to a plane with modifications that were to be analysed. After the polishing almost reached the location of the modifications, the final milling was performed by a FIB, after which the samples were "plucked-out" ex-situ. Again, platinum was deposited to prevent inadvertent damage during the ion beam milling. To stop the polishing at the right location, the progress was frequently checked using infrared microscopy.

Two types of modifications were created: separate single-pulse modifications (see figure 7.4a) and planes of closely spaced modifications (see figure 7.4b). The latter enables the influence of the laser beam interacting with previously modified material to be investigated. This matches the processing conditions that were used for wafer dicing. When producing closely spaced modifications, intermittent tracks were made (see figure $7.4 \mathrm{~b}$ ), to prevent the sample from breaking along the modifications that were to be analysed.

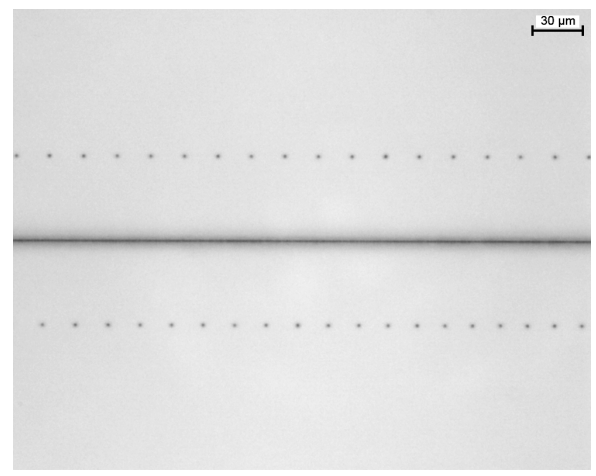

(a) Single-pulse modifications.

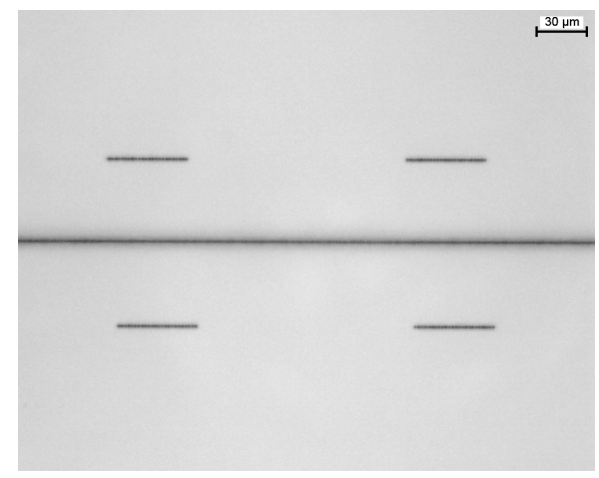

(b) Intermittent planes consisting of closely spaced modifications.

Figure 7.4: Transmission infrared micrographs of samples intended for the production of TEM lamellae by polishing (top view). The continuous line of subsurface modifications in the centre is used to fracture the samples, creating a starting plane for the polishing procedure. Wavelength: $1549 \mathrm{~nm}$, pulse energy: $2 \mu \mathrm{J}$.

An overview of the lamellae containing single-pulse modifications is shown in figure $7.5 \mathrm{a}$ The lamella numbers will be referred to when showing the corresponding analysis results. The lamellae were created at various positions along the optical axis, to assess whether the distance from the focus affects the structure of the modifications. A detail of a single lamella can be found in figure $7 \cdot 5 \mathrm{~b}$.

The lamellae containing closely spaced modifications were not "plucked-out". When several laser modifications are present in a single lamella, this may have a detrimental effect on its mechanical strength. Therefore, so-called "H-bar" lamellae were produced instead. 


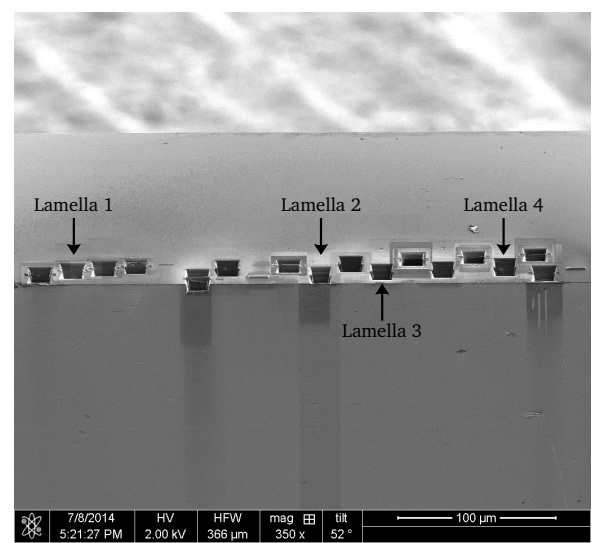

(a) Overview of lamellae including their numbers. The lamellae are close to the back surface of the wafer.

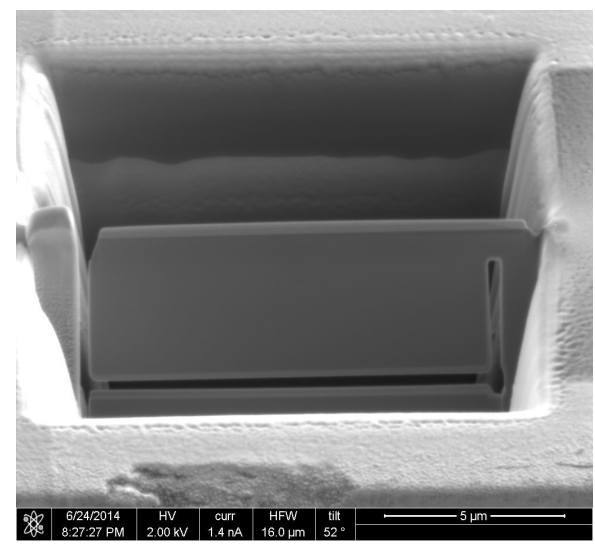

(b) Detail of a single lamella.

Figure 7.5: SEM micrographs showing TEM lamellae of single-pulse modifications prepared by a FIB. Each lamella contains a cross-section perpendicular to the optical axis, at a specific distance from the focus of the laser beam.

A detail of an "H-bar" lamella is shown in figure 7.6a, while an entire sample containing "H-bar" lamellae can be found in figure $7.6 \mathrm{~b}$. The thinned region is still connected to bulk material at three out of its four sides. Moreover, a subsequent plucking step is avoided. Consequently, this procedure helps to maintain the structural integrity of the lamellae.

During the production of lamellae perpendicular to the optical axis, their surface did not show any contrast when analysing them using SEM. Only after the TEM analyses, the presence of laser-induced modifications could be confirmed. As a significant amount of material had to be removed around the lamellae, only a single lamella could be extracted from each modification. To obtain cross-sections corresponding to different locations along the optical axis, several modifications had to be used.

\subsection{Scanning transmission electron microscopy}

In this section, scanning transmission electron microscopy (STEM) high-angle annular darkfield (HAADF) micrographs are presented. STEM was only employed for lamellae containing cross-sections along the optical axis of the laser beam. The use of STEM instead of conventional TEM is advantageous for these cross-sections, as they had to be kept relatively thick to maintain their structural integrity.

An STEM micrograph of two modifications is presented in figure $7 \cdot 7$. An area showing contrast with its surroundings is visible, implying that the corresponding material is in a modified state. Since the material in this area was found to be diamond cubic silicon with the same orientation as the base material (see section 7.5 ), lattice defects are responsible for the observed contrast. 


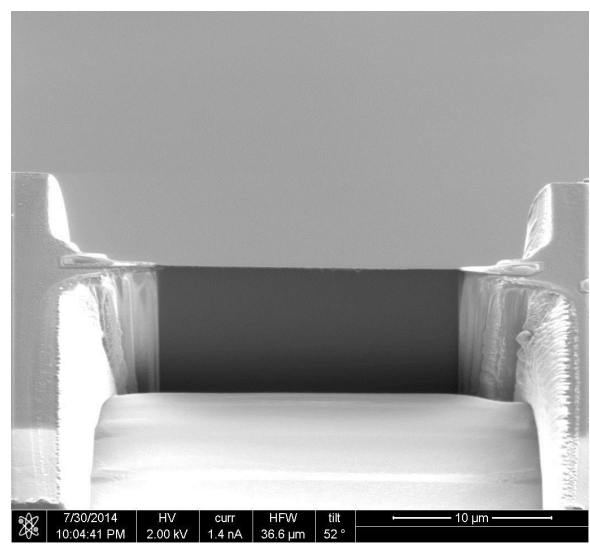

(a) Detail of a single "H-bar".

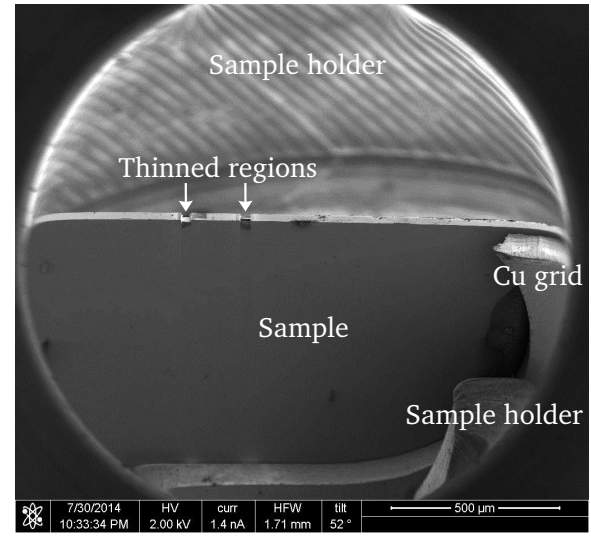

(b) Overview of an entire sample containing multiple "H-bar" lamellae.

Figure 7.6: SEM micrographs of TEM lamellae containing closely spaced modifications prepared by a FIB. This type of sample is referred to as "H-bar".

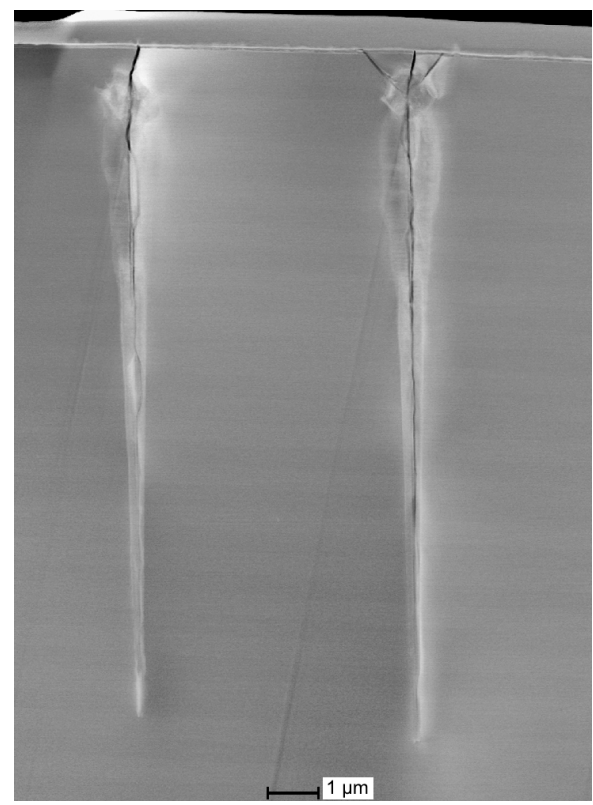

Figure 7.7: STEM-HAADF micrograph of two subsurface modifications obtained by stitching two images (top and bottom). Cross-section: along the optical axis. Transverse spacing modifications: $5 \mu \mathrm{m}$. Wavelength: $1549 \mathrm{~nm}$, pulse energy: $0.6 \mu \mathrm{J}$. The propagation direction of the laser beam is from top to bottom. 
Several cracks are present in the micrograph shown in figure 7.7 The crack that coincides with the optical axis of the laser beam was consistently present in all modifications. A detail of the top half of the right modification in figure 7.7 is shown in figure 7.8 . The irregular shape at the top of the modification might be caused by the proximity of the wafer surface.

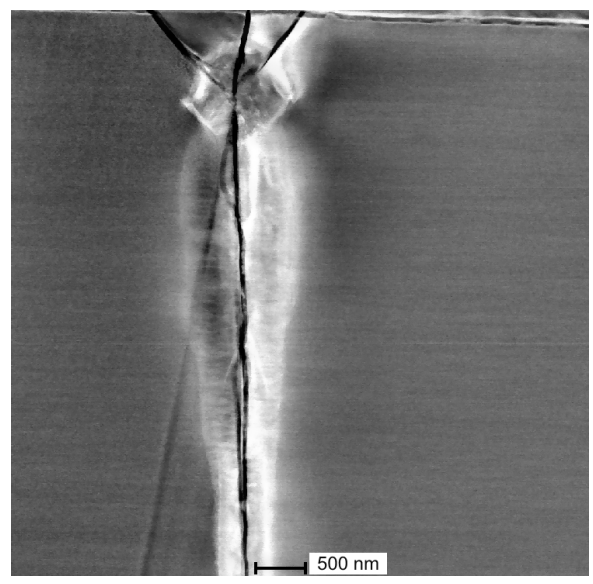

Figure 7.8: STEM-HAADF micrograph of the top half of the right modification in figure 7.7 Crosssection: along the optical axis. The image was filtered to remove scan lines. Transverse spacing modifications: $5 \mu \mathrm{m}$. Wavelength: $1549 \mathrm{~nm}$, pulse energy: $0.6 \mu \mathrm{J}$. The propagation direction of the laser beam is from top to bottom.

\subsection{Transmission electron microscopy}

In this section, the brightfield transmission electron micrographs obtained from the lamellae described in section 7.2 will be presented. The identification of the phase transformations that correspond to the features that are visible in the brightfield images will be discussed in sections 7.5 and 7.6 .

\subsubsection{Cross-sections along the optical axis}

In addition to STEM micrographs, conventional TEM micrographs of cross-sections along the optical axis were recorded. A TEM micrograph corresponding to the same area as the STEM micrograph in figure 7.8 can be found in figure $7.9 \mathrm{a}$. The material showing contrast in TEM mode is the same as in STEM mode, confirming that the micrographs presented in section 7.3 reveal all the laser-modified material. Additionally, a large number of bend contours are visible. Due to the thickness of the lamella and the presence of bend contrast, the outer contour of the subsurface modification is less clear compared with the STEM micrograph.

Figure $7.9 \mathrm{~b}$ shows a detail of the middle part of the same modification. The bottom part can be found in figure $7.9 \mathrm{c}$. Based on these micrographs, it was found that the crack along the optical axis, which is also visible in the STEM micrographs, coincides with the total length of the modified area. However, cracks that coincide with the optical axis 


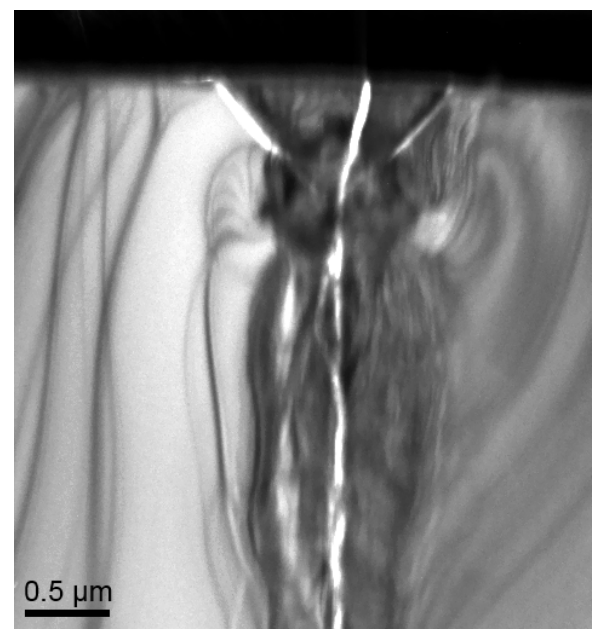

(a) Top part.

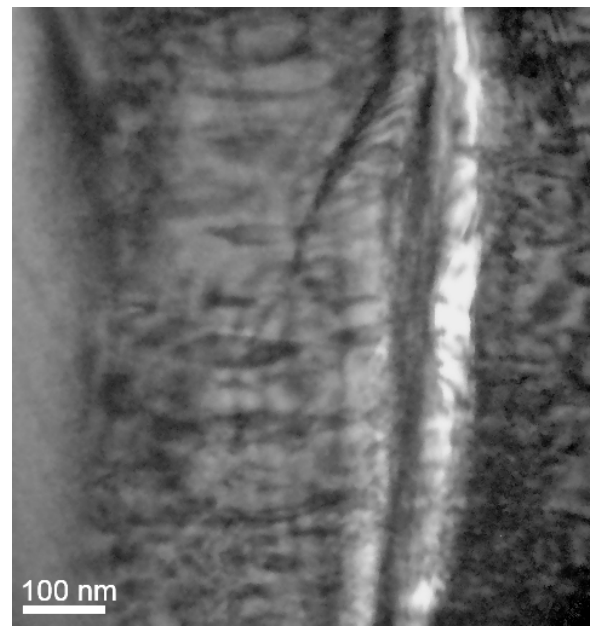

(b) Middle part.

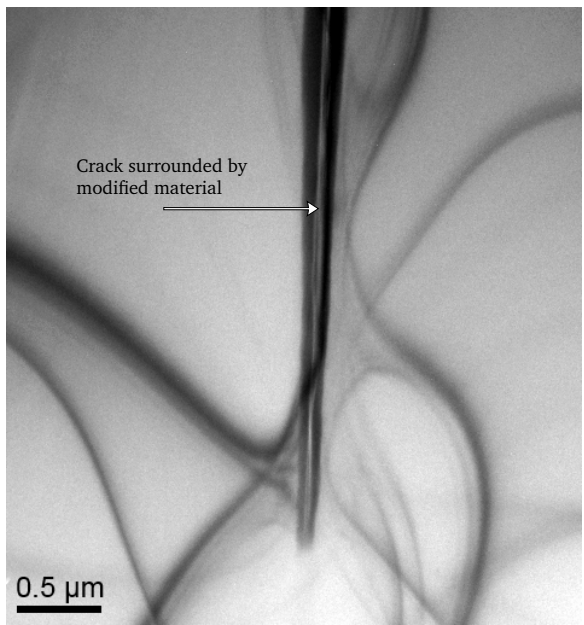

(c) Bottom part.

Figure 7.9: TEM micrographs of the right modification in figure 7.7 Cross-section: along the optical axis. Transverse spacing modifications: $5 \mu \mathrm{m}$. Wavelength: $1549 \mathrm{~nm}$, pulse energy: $0.6 \mu \mathrm{J}$. The irregular black lines outside the modification are bend contours. The propagation direction of the laser beam is from top to bottom. 
were not found during the analysis of cross-sections perpendicular to the optical axis (see section 7.4.2), implying that they are not a direct result of the laser-material interaction. Therefore, these cracks should be attributed to stress relief during the ion milling process.

\subsubsection{Cross-sections perpendicular to the optical axis}

In this section, the TEM results obtained from lamellae containing cross-sections perpendicular to the optical axis will be discussed. Both single-pulse modifications and closely spaced modifications are considered. The single-pulse modifications were placed at sufficiently large distances to prevent interaction between neighbouring modifications.

Single-pulse modifications Brightfield TEM micrographs of cross-sections obtained from positions close to the focus of the laser beam are shown in figures 7.10a, 7.10b and 7.11 These micrographs correspond to lamellae 2, 3 and 4 in figure $7.5 \mathrm{a}$ respectively. Each lamella contains a single modification. All micrographs include an edge, which is the plane where the polishing procedure was stopped. This edge can be used as a reference line for the orientation of the modifications. The polishing plane is approximately aligned with a $\{110\}$ plane.

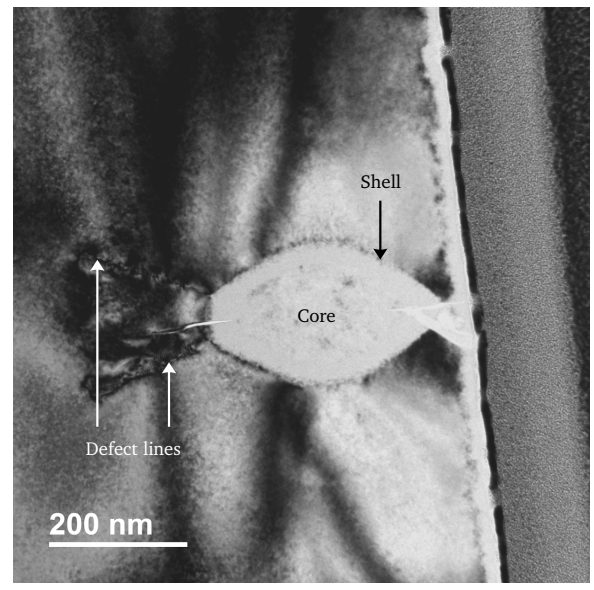

(a) Lamella 2 (location: close to the focus).

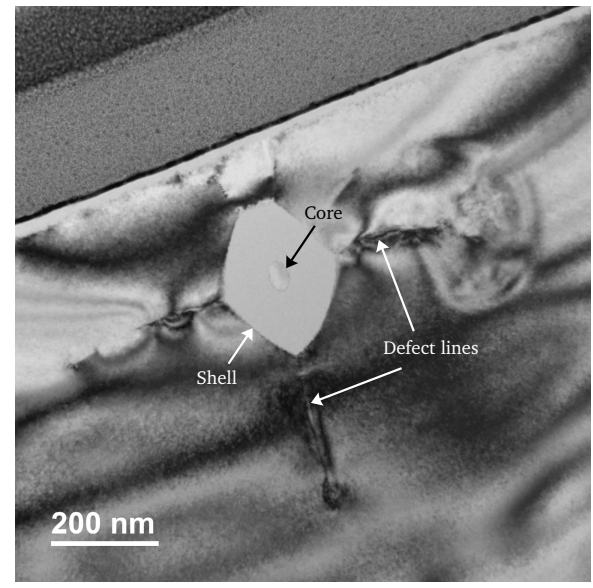

(b) Lamella 3 (location: $2 \mu \mathrm{m}$ further away from the focus compared with lamella 2).

Figure 7.10: TEM micrographs of single-pulse modifications. Cross-section: perpendicular to the optical axis. Wavelength: $1549 \mathrm{~nm}$, pulse energy: $2 \mu \mathrm{J}$.

The cross-sections perpendicular to the optical axis contain several of the following features (see figure 7.12):

1. A circular core located on the optical axis.

2. A shell surrounding the core that frequently has a square pattern. 


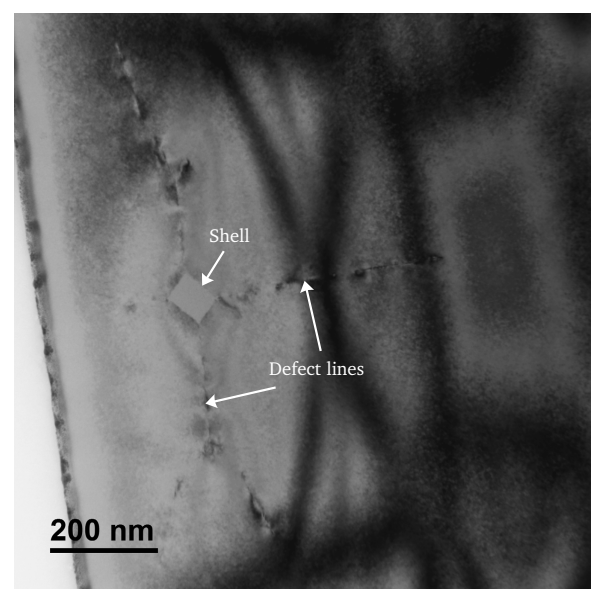

Figure 7.11: TEM micrograph of a single-pulse modification (lamella 4). Cross-section: perpendicular to the optical axis. Location: $6 \mu \mathrm{m}$ further away from the focus compared with lamella 2. Wavelength: $1549 \mathrm{~nm}$, pulse energy: $2 \mu \mathrm{J}$.

3. Defect lines radiating out from the corners of the shell.

4. Unmodified material with the same crystal structure and orientation as the unprocessed wafer.

\section{Bend contours.}

Features (1), (2) and (3) are also indicated in the brightfield micrographs. The probable causes of the formation of these features will be discussed in section 7.7. Holes corresponding to the cracks along the optical axis, which were observed on the micrographs shown in section 7.4.1 were not detected. Note that the presence of unmodified material does not imply that the material was not in a modified state during or shortly after the laser pulse. The sample analysis only provides information about permanent modifications after the laser process.

The modification in lamella 3 (see figure 7.10b) contains all features discussed above. Lamella 4 (see figure 7.11) is missing the core while lamellae 2 (see figure 7.10a) and 3 both contain a core. However, the core of lamella 3 has a homogeneous crystal structure while the core of lamella 2 is inhomogeneous. The fact that the shapes of the observed features do not correspond to the spatial profile of the laser beam, implies that they are related to the crystal structure of silicon. The features generally have a consistent orientation with respect to the edges of the lamellae, irrespective of their location along the optical axis. Consequently, the crystal planes that the features coincide with can be identified, in spite of the fact that the micrographs only show two-dimensional cross-sections. The defect lines are most commonly aligned with a $\{110\}$ plane, although several variations can be observed. In figure 7.11, the bottom defect line splits in two different directions. The edges of the shells generally coincide with $\{100\}$ planes. 


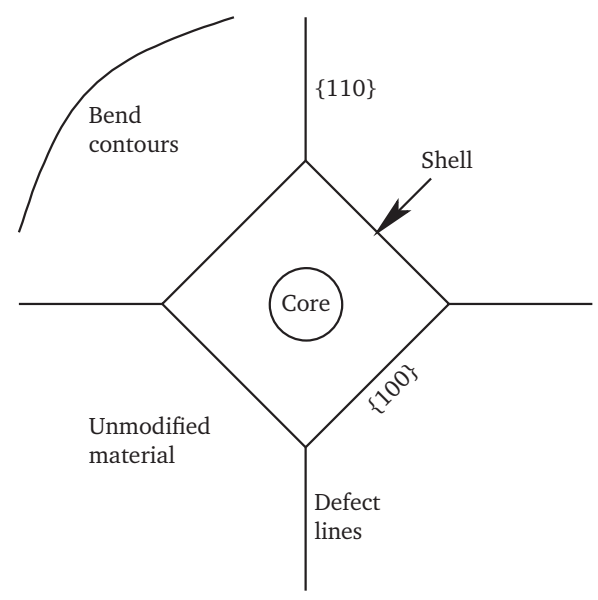

Figure 7.12: Drawing of the features observed by TEM in lamellae containing cross-sections perpendicular to the optical axis.

As mentioned before, a single modification could only be used to produce a single cross-section (see section 7.2.2). Therefore, it was not possible to study the influence of the location along the optical axis, without analysing another modification. Based on the analysis of ten of the lamellae shown in figure $7.5 \mathrm{a}$ it was found that the differences between the micrographs presented in this section are mainly due to stochastic variations and not due to the positions of the cross-sections along the optical axis.

In figure 7.13, a TEM micrograph of lamella 1 is shown that corresponds to a cross-section at the same distance from the focus as lamella 3. This lamella is especially of interest because the area labelled as "phase transformed region", which is located along one of the defect lines, was found to contain high-pressure phases. In section $7 \cdot 5$, the analysis of the crystal structure of this region by selected area diffraction will be presented.

Closely spaced modifications Apart from single-pulse modifications, cross-sections containing modifications spaced $2 \mu \mathrm{m}$ apart were analysed. This value corresponds to the required spacing to reliably dice wafers (see section 6.7). Two brightfield micrographs of such modifications are shown in figures $7.14 \mathrm{a}$ and $7.14 \mathrm{~b}$. These modifications were located in a single lamella, ensuring that they were obtained from exactly the same location along the optical axis. Nevertheless, relatively large differences between both modifications are visible. The modification in figure $7.14 \mathrm{a}$ contains a shell with amorphous silicon, while the modification in figure $7.14 \mathrm{~b}$ shows crystalline silicon with defects at the same location (see section 7.5).

The closely spaced modifications are similar to the single-pulse modifications. However, compared with single-pulse modifications, closely spaced modifications were found to contain higher concentrations of cracks and lattice defects. An example of a crack can be found in figure $7.14 \mathrm{~b}$. This crack is located along a defect line, which was the case for the majority of the cracks. 


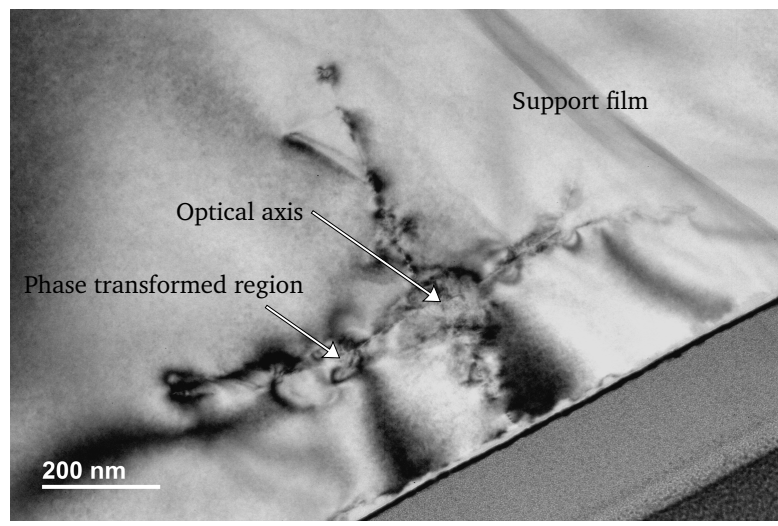

Figure 7.13: TEM micrograph of a single-pulse modification (lamella 1). Cross-section: perpendicular to the optical axis. Wavelength: $1549 \mathrm{~nm}$, pulse energy: $2 \mu \mathrm{J}$. The location labelled as "phase transformed region" contains high pressure phases.

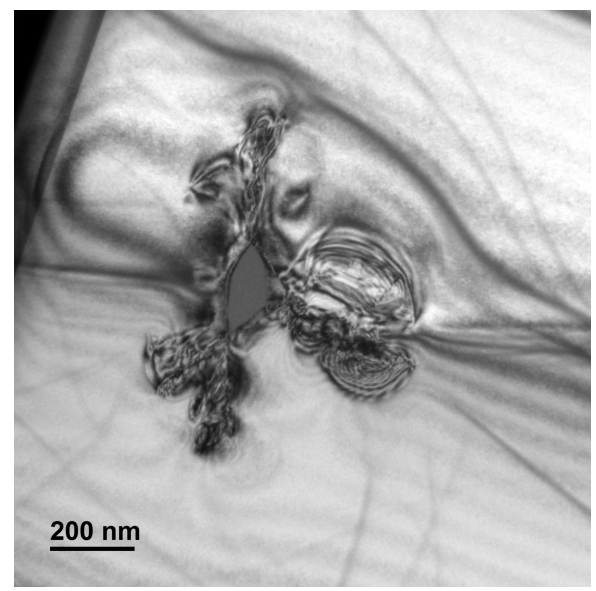

(a) Modification containing a shell that consists of amorphous silicon.

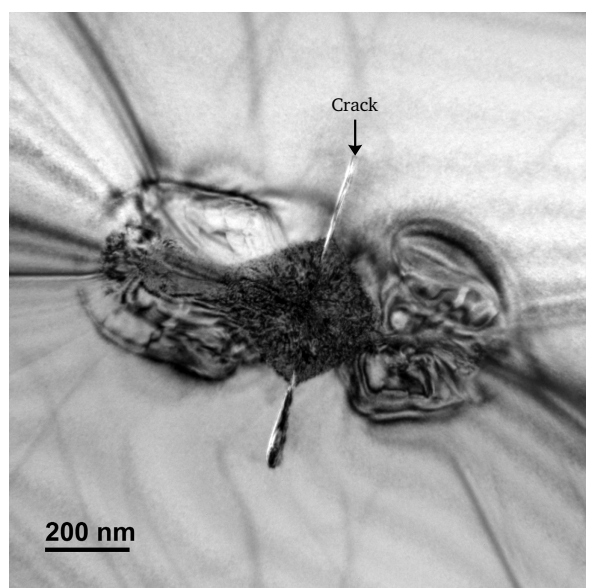

(b) Modification containing a shell that consists of crystalline silicon with defects.

Figure 7.14: TEM micrographs of closely spaced modifications. Cross-section: perpendicular to the optical axis. Transverse spacing modifications: $2 \mu \mathrm{m}$. Wavelength: $1549 \mathrm{~nm}$, pulse energy: $2 \mu \mathrm{J}$. 


\subsection{Selected area diffraction}

To identify the crystal structure of the laser modifications, selected area diffraction (SAD) patterns were recorded. All diffraction patterns corresponding to cross-sections along the optical axis were found to be identical to the unmodified material, i.e. only Si-I was present with a consistent orientation of the crystal. Nevertheless, significant contrast was visible in STEM and TEM micrographs (see sections 7.3 and 7.4.1). Therefore, the only plausible cause of this contrast is the presence of a high density of defects.

A larger variety of material structures was found for the cross-sections perpendicular to the optical axis. There are two possible explanations for this difference: (1) a higher pulse energy was used for the cross-sections perpendicular to the optical axis, as there was no limitation on the modification length for the lamella preparation and (2) the material with a different structure than Si-I may have been removed during the ion beam milling of the lamellae containing cross-sections along the optical axis.

Core and shell The SAD results showed that the shell, which was consistently observed on cross-sections perpendicular to the optical axis, either consists of amorphous silicon or diamond cubic silicon with defects with an orientation that is consistent with the original material. Which type of modified material is present can be directly identified from the brightfield micrographs presented in section $7 \cdot 4.2$. The shapes that show uniform contrast are amorphous, while the shapes that contain defects are crystalline. This difference is clearly visible when comparing e.g. figures $7.14 \mathrm{a}$ and $7.14 \mathrm{~b}$.

If a core was present, it contained monocrystalline silicon with a different orientation compared with the original wafer or polycrystalline silicon. This crystalline silicon was sometimes found to be mixed with amorphous silicon. Cores were only detected when the shell consisted of amorphous silicon.

Two SAD patterns obtained from the shell and unmodified material and core and shell of lamella 3 are shown in figures $7.15 \mathrm{a}$ and $7.15 \mathrm{~b}$ respectively. The corresponding brightfield micrograph can be found in figure $7.10 \mathrm{~b}$. The core consists of a single crystal of diamond cubic silicon. However, its orientation is different compared with the unmodified material. The shell contains amorphous silicon, resulting in rings in the diffraction pattern.

The analysis of the core of lamella 2 is shown in figure 7.16 . Besides amorphous silicon, a large number of weak spots are present originating from nanometre-scale crystals. The nanocrystals are consistent with the irregular appearance of the core in the corresponding brightfield micrograph (see figure 7.10a).

Defect lines High pressure phases were found in two modifications. In both cases, these phases were present along one of the defect lines. Lamella 1 is one of the lamellae that contained high pressure phases. The brightfield micrograph of this lamella can be found in figure 7.13. The area that was used to generate the diffraction pattern is labelled as "phase transformed region" in the brightfield image and is located at about $200 \mathrm{~nm}$ from the optical axis. The SAD pattern of this region is shown in figure 7.17

Several diffraction spots belonging to Si-I with the same orientation as the unmodified material are directly identified in figure 7.17 . Spots which are not related to the base 


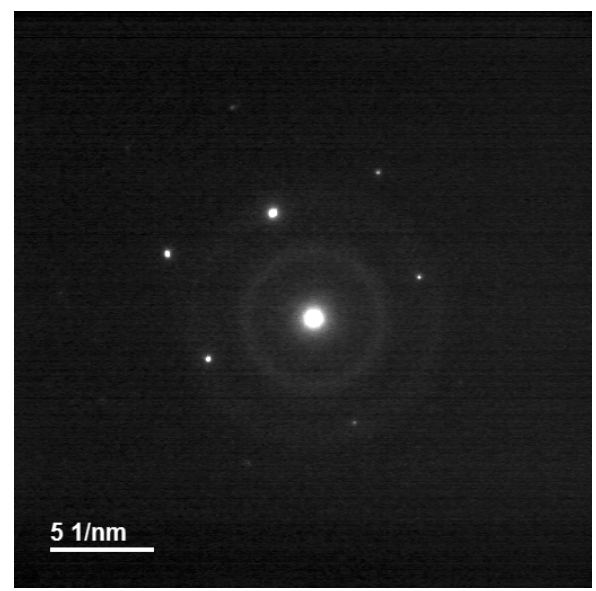

(a) Pattern of the shell and unmodified silicon. Both amorphous silicon and Si-I are visible.

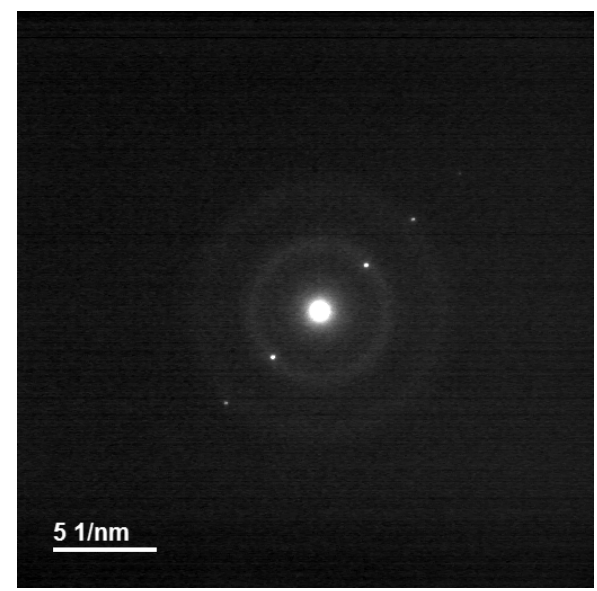

(b) Pattern of the core and shell. Both amorphous silicon and Si-I are visible. However, the crystalline silicon has a different orientation compared with the unmodified material shown in figure (a).

Figure 7.15: SAD patterns of lamella 3 (see figure 7.10b).

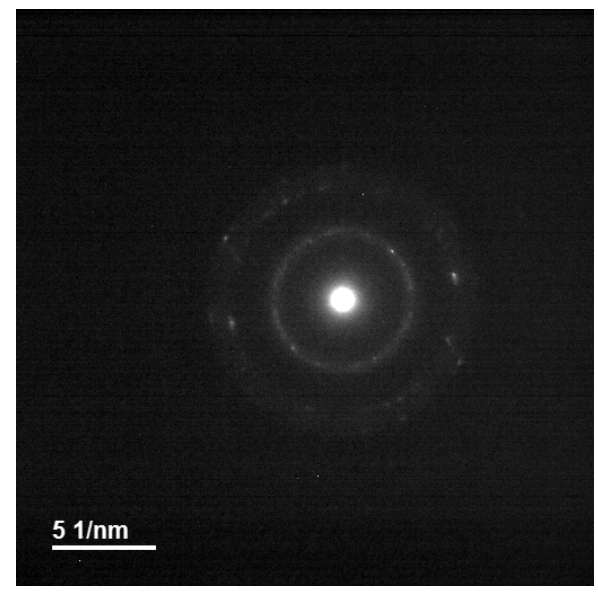

Figure 7.16: SAD pattern of the core of lamella 2 (see figure 7.10a). Amorphous silicon is present. Moreover, many weak spots originating from nanocrystals can be observed. 


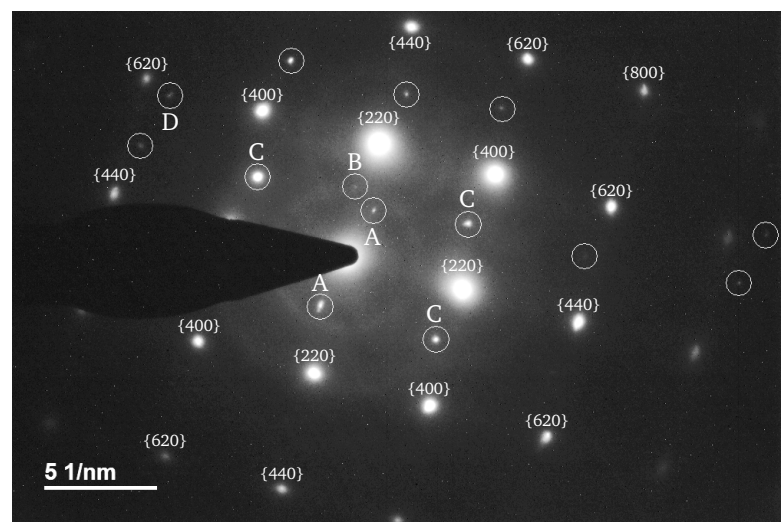

Figure 7.17: SAD pattern of the area labelled as "phase transformed region" in figure 7.13 The spots for which the Miller indices are given correspond to Si-I with the same orientation as the base material. The circled spots belong to a different crystal orientation or structure.

material are circled. From these spots, the ones that match a limited number of lattice planes are marked by letters. Since spot D is located at twice the distance from the undiffracted beam compared with spot $\mathrm{c}$, along the same line starting from the undiffracted beam, spot D corresponds to higher order diffraction from the same set of lattice planes. Darkfield images were recorded from the diffraction spots marked by letters. Based on these images, it was found that the phase transformed region does not have a homogeneous crystal structure, but consists of a mix of different phases and/or crystal orientations.

Except for the unmodified material and spots $\mathrm{C} / \mathrm{D}$, the number of detectable diffraction spots was insufficient to directly identify the lattice structure of the corresponding phases. The identification of spots A and в was based on comparing the measured spacings between lattice planes with data of known crystalline phases of silicon. The distances between the diffraction spots and the undiffracted beam, as measured using the scale bars shown in the figures, correspond to the reciprocal of the spacing between lattice planes. For spots C/D, additional symmetry information was available to identify the lattice structure.

The possible phases and lattice planes that match the spots that are marked by letters, within the measurement uncertainty, are listed in table 7.1. Since residual stresses beyond approximately $10 \mathrm{GPa}$ are unlikely to occur in a thin lamella, phases which require higher residual stresses were classified as unlikely. Moreover, based on the concentrations of the different phases as observed on the darkfield images, certain phases are a more likely match than others. High pressure phases are expected to be more localised than the presence of Si-I, which is the stable phase of silicon at atmospheric pressure. Finally, phases that match the Raman spectroscopy data (see section 7.6) are likely to be the correct match, as it is known that these phases are present in the samples.

Spots C/D match Si-I with a large degree of certainty. Spot B may also be due to the presence of Si-I with a different orientation compared with the unmodified material, although there are also matching high pressure phases. For spots A, no match with Si-I was identified, which implies that a high pressure phase was formed. This result was 
Table 7.1: Analysis of the additional spots in the SAD pattern shown in figure 7.17 The data on the spacings between lattice planes of the phases of Si originates from a database of the Department of Electronic Materials Engineering of the Australian National University.

\begin{tabular}{llll}
\hline Phase & Planes & \multicolumn{1}{c}{ Spacing $(\AA)$} & Assessment \\
\hline A spots. & Measured spacing between adjacent lattice planes: $4.23 \AA$. \\
\hline Si-XII & $\{100\}$ & 4.492 & Most likely, consistent with Raman spectroscopy. \\
Si-XIII & $\{101\}$ & 4.392 & Possible. \\
Si-VIII & $\{200\}$ & 4.314 & Unlikely, limited data in literature on this phase. \\
\hline B spot. Measured spacing between adjacent lattice planes: $3.13 \AA$. \\
\hline Si-I & $\{101\}$ & 3.135 & Possible, low concentration for Si-I. \\
Si-II & $\{110\}$ & 3.316 & Unlikely, unrealistic residual stresses. \\
Si-III & $\{200\}$ & 3.320 & Most likely, consistent with Raman spectroscopy. \\
Si-IV & $\{002\}$ & 3.14 & Possible. \\
Si-XII & $\{111\}$ & 3.213 & Possible. \\
\hline C/D spots. Measured spacing between adjacent lattice planes: $1.89 \AA$. \\
\hline Si-I & $\{220\}$ & 1.92 & Spots match Si-I observed along < $111>$ axis. \\
\hline
\end{tabular}

confirmed by Raman spectroscopy (see section 7.6 ). When assuming that the known pathways of phase transformations in silicon have been responsible for the formation of the high pressure phases, the pressure induced by the laser process must have exceeded 8.8 GPa (see section 2.2).

\subsection{Raman spectroscopy}

In addition to SAD, Raman spectroscopy was used to identify the phases that are present in the laser-modified material. To produce samples for Raman spectroscopy, polishing was employed to expose modifications that were generated using $3.5 \mathrm{~ns}$ pulses with a wavelength of $1549 \mathrm{~nm}$ and a pulse energy of $2 \mu \mathrm{J}$. The advantage of polishing over fracturing is the superior flatness of the exposed plane. A reference spectrum was recorded of unmodified material, which is shown as a solid line in figure 7.18 .

Both single-pulse modifications and closely spaced modifications with a transverse spacing of $2 \mu \mathrm{m}$ were analysed. No high pressure phases were found during the analysis of single-pulse modifications, which is inconsistent with the SAD results (see section 7.5). This is probably caused by the limited number of modifications that were sampled.

When analysing tracks of closely spaced modifications, phase-changed material was consistently found, with no clear correlation to the location along the optical axis. A 


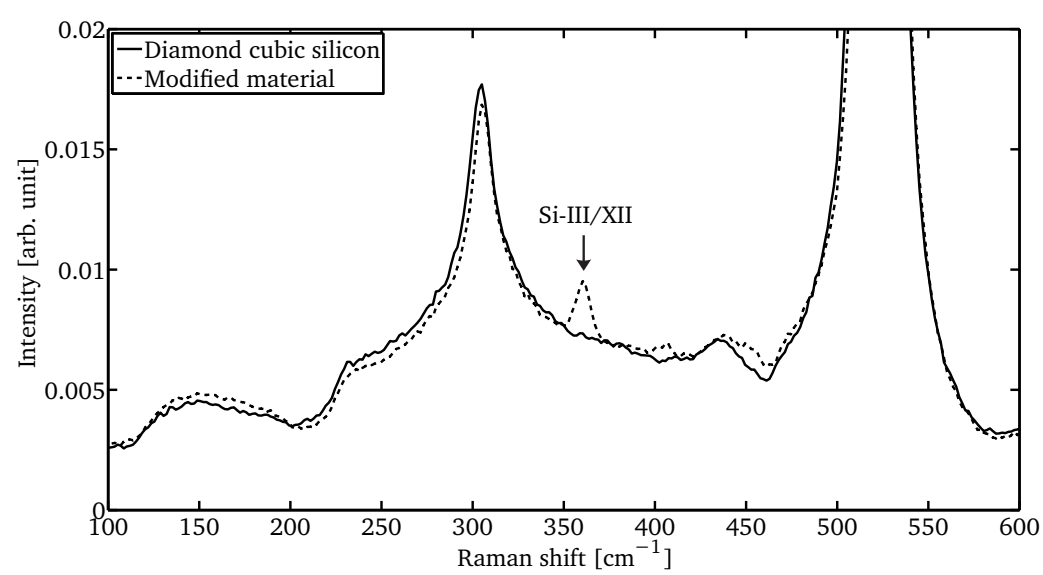

Figure 7.18: Raman spectra obtained from unmodified Si-I and laser-modified material. The intensity is shown on the vertical axis in an arbitrary unit; the values were scaled such that the highest peak is equal to one. For clarity, one of the Si-I peaks at $521 \mathrm{~cm}^{-1}$ is not fully shown.

spectrum recorded near the location of the focus of the laser beam is shown as a dashed line in figure 7.18. A strong peak is observed around a Raman shift of $36 \mathrm{o} \mathrm{cm}^{-1}$, which indicates the presence of Si-III/Si-XII [80]. It was not possible to distinguish between both phases due to the similarity of their Raman spectra. Previously, both phases were found to coexist in nanoindentation experiments [80]. Consequently, it is likely that this is also the case for the phase changed silicon that was found in laser-induced subsurface modifications. The presence of both Si-III and Si-XII is consistent with the SAD results (see table 7.1).

\subsection{Modification mechanisms}

One of the purposes of the analysis of the crystal structure presented in this chapter, is to assess what modification mechanisms contributed to the formation of the subsurface modifications. In this section, these modification mechanisms will be considered.

\section{Melting and resolidification}

The brightfield TEM micrographs presented in section 7.4.2 show non-axisymmetric features including defect lines and a shell. Since the transverse intensity profile of the laser beam was axisymmetric, this implies that anisotropic behaviour of the material determines the crystal structure after laser processing. The following interpretation of the features observed on the TEM micrographs, in terms of the modification mechanisms that contributed to their formation, is based on the features that are drawn in figure 7.12. While this figure corresponds to the dominant orientation of these features, their orientation was not always consistent due to the presence of stochastic variations.

A drawing of the features observed on a cross-section perpendicular to the optical axis, including the probable causes of their formation, is shown in figure 7.19 . The shell is 
expected to be formed due to the preferential directions of crystal growth from a melt. Epitaxial regrowth seeded by the unmodified material can explain why the material outside the shell is identical to the unmodified material. As a result of such perfect epitaxial silicon, it was not possible to determine the outer extent of the melt front with certainty. Therefore, a lower estimate of its penetration depth at the extremities of the defect lines is provided in figure 7.19

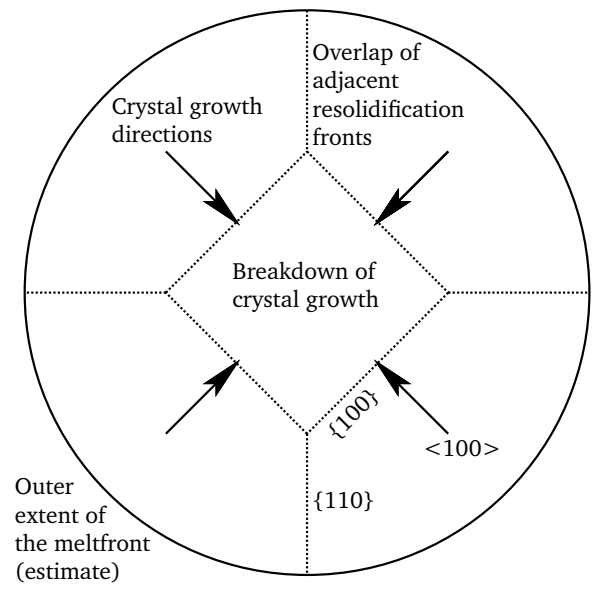

Figure 7.19: Drawing of the shape of a cross-section of a subsurface modification perpendicular to the optical axis. The probable causes of the different features are indicated.

Closer to the optical axis, the solidification speed is too high to enable the material to regain a defect-free crystalline state. The maximum velocity for defect-free crystal growth is between 5 and $15 \mathrm{~m} / \mathrm{s}$, depending on the growth direction [102]. When this velocity is exceeded, a shell consisting of crystalline silicon with defects or amorphous silicon is formed. Amorphous silicon was only found in the vicinity of the focus of the beam. This is likely to be caused by the fact that the highest cooling rates are obtained near the focus, as the best confinement of the laser energy absorption is achieved at this location.

Solidification was found to predominantly occur along $<100>$ directions. These directions correspond to the most efficient growth directions of crystalline silicon from a melt [102]. The threshold for defect-free crystal growth along a $<100>$ direction is $15 \mathrm{~m} / \mathrm{s}$ [102]. The defect lines frequently coincide with the overlap of adjacent $\{100\}$ solidifications fronts.

Since there is no preferential direction for the growth of amorphous material, the crystalline cores that were sometimes observed inside shells consisting of amorphous silicon are axisymmetric. The presence of crystalline cores implies that the solidification speed was reduced near the optical axis. This might be caused by the lower thermal conductivity of amorphous silicon near room temperature [175], compared with diamond cubic silicon [149].

A recent molecular dynamics study of the solidification of a subsurface melt and vapour in silicon, predicted both recrystallization and amorphization [176]. This provides supporting 
evidence that a thermal damage mechanism can explain the features that were observed by TEM.

The fact that melting and resolidification was found to be an important material modification mechanism, explains why simulations of subsurface melting were capable of predicting the existence and length of the modifications with reasonable accuracy (see chapter 6). This mechanism was included in the numerical model (see section 3.2.6).

\section{Pressure-induced phase changes}

Based on the SAD and Raman spectroscopy data presented in sections 7.5 and 7.6 , pressureinduced phase transformations were found to be a contributing damage mechanism. High pressure phases were detected along one of the defect lines shown in figure 7.19. As these lines are expected to originate from the overlap of adjacent resolidification fronts, this implies that the silicon at the location where the high pressure phases are present has been molten. Since resolidification is a relatively slow process (see section 2.5), the formation of the high pressure phases presumably occurred after the laser pulse. Therefore, it is unlikely that a confined microexplosion induced by the laser pulse, to which the formation of high pressure phases during previous experiments on dielectric materials has been attributed (see section 2.6), has been responsible for their formation.

According to the phase diagram of silicon [177], Si-II can be directly formed from the liquid phase, when pressures similar to the required pressures for solid-solid phase transitions are present. Si-II will transform into Si-III/Si-XII upon (partial) release of the pressure (see section 2.2). Consequently, the formation of high pressure phases from a melt is plausible. A possible explanation for the generation of high pressures is that liquid silicon was trapped by surrounded material that had already been resolidified. Since liquid silicon is denser than diamond cubic silicon [178], this would result in insufficient space for the silicon to recrystallize in its diamond cubic form.

The numerical model can only accurately compute pressures while the material is in the solid phase. Since the high pressure phases were found at locations where the silicon has been molten, these locations were already predicted to be in a modified state by the model. Therefore, the presence of these phases does not invalidate the simulated shapes of the subsurface modifications.

\section{Formation of voids}

Voids were detected along the optical axis during the analysis of fracture planes (see section 6.8. . However, they were not identified in TEM lamellae, which poses the question whether the voids are a direct result of the laser-material interaction. Since the voids that were found on fracture planes were randomly distributed, their absence from the TEM lamellae that were produced may be a matter of chance.

Matching voids were generally found on both sides of the fracture planes. Moreover, previous measurements by infrared laser scanning confocal microscopy suggested that the voids were already present before fracturing [55]. Based on the above, the voids are likely to be a direct result of the laser-material interaction, although the possibility that nanometre-scale particles escaped from the sample during fracturing cannot be ruled out.

The excess material due to the void formation has to be accommodated for, either by (1) an increase in density elsewhere in the bulk of the material or (2) deformation of the 
outer contour of the sample. No evidence for effect (2) was found during the analysis of laser-processed samples. An increase in density could be caused by the presence of strain or by a transformation of Si-I to a denser packed phase. Si-III/Si-XII phases, which were found to be present in the laser-processed samples (see section 7.6), have a density of approximately $2.55 \cdot 10^{3} \mathrm{~kg} / \mathrm{m}^{3}$ at atmospheric pressure [106], compared with $2.33 \cdot 10^{3} \mathrm{~kg} / \mathrm{m}^{3}$ for Si-I (see section 3.5). High density amorphous silicon can also accommodate for the excess material due to the presence of voids.

\subsection{Summary}

Electron-transparent lamellae were produced by focussed ion beam milling and mechanical polishing for analysis by transmission electron microscopy. These lamellae contained crosssections along or perpendicular to the optical axis. The transmission electron microscopy results are consistent with the result from the simulations that melting and resolidification is the primary damage mechanism, as anisotropy related to preferential solidification directions was detected.

Several types of modified silicon were found, namely diamond cubic silicon with a changed orientation, diamond cubic silicon with a high concentration of defects, amorphous silicon and high pressure phases. Variations between different modifications were detected, in spite of the use of identical processing conditions. To identify the material structure of the subsurface modifications, selected area diffraction and Raman spectroscopy were employed. Both analysis techniques confirmed the presence of one or more high pressure phases. Based on the Raman spectroscopy results, Si-III/Si-XII phases were found to be present.

The fact that high pressure phases were detected implies that pressures exceeding 8.8 GPa have been induced by the laser process, if the phase transformations followed one of the known pathways. The high pressure phases were found at a distance of about $200 \mathrm{~nm}$ from the optical axis, where the silicon has presumably undergone melting and resolidification. Therefore, a different mechanism than a confined microexplosion is likely to be responsible for their formation. 


\section{Conclusions and recommendations}

In this chapter, the answers to the research objectives listed in section 1.6 will be summarized. Moreover, suggestions for future research are given.

\subsection{Conclusions}

Objective: to investigate the suitability of ultra-short pico- to femtosecond pulses for the formation of subsurface modifications in crystalline silicon.

The shortest pulses that were successfully used to generate subsurface modifications in silicon had a pulse duration of $2 \mathrm{~ns}$. The research on creating bulk modifications using $6.6 \mathrm{ps}$ pulses with a wavelength of $1030 \mathrm{~nm}$ had a negative outcome, which is consistent with recent studies employing femtosecond pulses with moderate pulse repetition frequencies [62, 63].

Excessive two-photon absorption above the focus was identified as the cause for the failure of using picosecond laser pulses. The process boundary for the pulse energy is governed by the required energy to melt a volume of material below the surface. For a fixed pulse energy, the instantaneous power of the laser beam will eventually become too high to confine the two-photon absorption to the vicinity of the focus, when decreasing the pulse duration. This effect becomes relevant at power levels below the critical power for self-focussing due to the Kerr effect.

Two-photon absorption at undesired locations does not only result in a direct loss of laser energy. The photo-ionized carriers further enhance the laser energy absorption. Moreover, plasma-induced defocussing of the laser beam will occur, preventing the laser energy from being selectively absorbed near the geometrical focus of the beam. Plasma defocussing is caused by the dependence of the refractive index on the density of electron-hole pairs.

Objective: to develop a multiphoton subsurface modification method for crystalline silicon, which is capable of producing modifications near the focus of the laser beam.

Based on the results of numerical simulations, it was found that previously reported difficulties to create modifications at any desirable depth inside a silicon wafer [62, 66], were not due to a physical limitation. However, it was found that a limited window for the pulse duration exists, depending on the wavelength and the parameters of the optical system including the focussing objective. By combining a relatively long pulse duration of $3.5 \mathrm{~ns}$ with a wavelength of $1549 \mathrm{~nm}$, multiphoton-induced subsurface modifications were successfully created at various focus depths.

Of particular interest is the result that nanosecond pulsed erbium-doped fibre lasers, operating in the optical communications C-band, perform satisfactorily for the multiphoton process. This wavelength window is ubiquitous in telecommunications, resulting in a good availability of components. 
The multiphoton process has been compared with the use of a photon energy near the band gap of silicon. Advantage of exclusively employing multiphoton absorption for the generation of an initial electron-hole plasma, is that free carriers will not be created throughout the entire path of the laser beam inside the silicon wafer. Hence, energy losses and plasma-induced defocussing of the laser beam can be prevented. Additionally, based on simulation results, the multiphoton process is expected to be more robust when varying the focus depth or running the laser source at high pulse repetition frequencies.

Objective: to develop a numerical model to simulate the laser-material interaction inside crystalline silicon that is sufficiently accurate to select suitable laser processing conditions.

A model was proposed that is based on a two-temperature model to simulate the response of the material to the absorption of laser energy and a nonlinear Schrödinger equation to model the propagation of the laser beam.

The numerical results showed that for the processing conditions that were investigated, thermal melting and subsequent resolidification is likely to be the primary damage mechanism, implying that an energy density of $6.97 \cdot 10^{-3} \mu \mathrm{J} / \mu \mathrm{m}^{3}$ is required for the formation of a subsurface modification.

The model was capable of predicting which laser sources are suitable for the formation of subsurface modifications. Moreover, a reasonable agreement between the numerical predictions of the modification geometry and the actual shapes was found.

Objective: to develop an experimental set-up to produce subsurface modifications in crystalline silicon using different processing conditions.

An experimental set-up was developed to focus laser pulses inside silicon samples. Four solid state lasers were incorporated in the set-up, to collect experimental data using different processing conditions. Two of these sources were fibre lasers, as they provide pulses with a sufficiently high pulse energy and have an excellent beam quality. A microscope objective was selected to focus the laser beam, which combines a relatively high numerical aperture with a reasonable working distance.

Wavelengths of 1061, 1064 and $1549 \mathrm{~nm}$, together with pulse durations of 2-46o, 8 and 3.5 ns respectively, were all found to be suitable for the synthesis of subsurface modifications. These conditions cover single-photon absorption, two-photon absorption and a combination of both. The pulse energies varied between a few tenth of a microjoule and several microjoules, depending of the choice of the pulse duration and the desired size of the modifications. The required laser pulses for all processes that were investigated in this work can be generated by common industrial laser sources. This makes it economically feasible to integrate the laser processes in industrial equipment.

Objective: to develop analysis methods to identify the geometry and material structure of subsurface modifications in crystalline silicon.

It was found that infrared microscopy is suitable to detect the presence of subsurface modifications in a non-destructive manner. A cT-scan of subsurface modifications did not show any contrast. To obtain quantitative information about the shape of the modifications, 
fracture planes containing modifications were analysed by optical microscopy, laser scanning confocal microscopy and scanning electron microscopy.

To acquire detailed information about the material structure of the subsurface modifications, electron-transparent lamellae were produced by ion beam milling and mechanical polishing. These lamellae were analysed by transmission electron microscopy. The analysis results were consistent with the result from the numerical simulations that melting and resolidification is the primary damage mechanism.

The types of modified material that were found are amorphous silicon, crystalline silicon with defects or a changed orientation and high pressure phases. The high pressure phases were identified by Raman spectroscopy as Si-III/Si-XII. These phases were detected at a location where the material has presumably been molten during the laser pulse, implying that a different mechanism than a confined microexplosion is responsible for their formation. A hypothesis is that liquid silicon, which has a higher density than diamond cubic silicon, was trapped during the solidification process. Consequently, insufficient space is available for the liquid silicon to recrystallize to its original diamond cubic structure. Within the known pathways of phase transformations in silicon, the presence of high pressure phases implies that the pressure during the laser process has exceeded $8.8 \mathrm{GPa}$.

Objective: to assess the suitability of subsurface modifications, obtained using different processing conditions, for the dicing of crystalline silicon wafers.

It was found that the subsurface modifications that were produced, using all processing conditions which were investigated, can be employed for wafer dicing. The geometry and positioning of the modifications had a large influence on the outcome of the dicing process. Suboptimal processing conditions cause the fracture plane to switch to a preferential cleavage plane of the silicon crystal. The best kerf geometry was obtained when the laser modifications cover a large part of the intended fracture plane.

Pulses of several hundred nanoseconds combined with a wavelength of $1061 \mathrm{~nm}$, were found to result in the formation of large subsurface cracks. Such cracks have a positive effect on the required separation force. However, since some of these cracks will also be present near the edges of the separated dies, they are expected to have a negative impact on the die strength.

Based on the analysis of fracture planes, the functions of the modifications during the dicing process were identified. That is, the modifications initiate crack growth inside the wafer and are likely to guide the propagation of cracks along the desired fracture plane.

\subsection{Recommendations}

A number of opportunities for future work have been established. These include recommendations to improve the accuracy of the results presented in this thesis, to extend the parameter range that was investigated and to investigate applications of subsurface modifications beyond wafer dicing. An overview of the recommendations is given below.

\section{Improve the usability of ultra-short pulses}

It is worthwhile to investigate methods to improve the usability of ultra-short pulses for the production of modifications inside silicon, to establish whether such pulses result in 
different modifications compared with a nanosecond process. Pulses of a few nanoseconds are short enough to limit the effect of diffusion during the pulse. However, ultra-short pulses could allow for higher pressures to be generated or induce non-thermal melting.

A number of solutions may be investigated to improve the confinement of the energy absorption during subsurface processing with ultra-short pulses: (1) to use a focussing objective with a higher numerical aperture than $0.7,(2)$ to employ higher-order nonlinear absorption than two-photon absorption and (3) to create a temporal confinement of the laser pulse.

Solution (1) is related to the fact that the laser intensity above and below the focus will decay faster when using a higher numerical aperture. This results in less multiphoton absorption of laser energy outside the focus.

To implement solution (2), one could consider increasing the wavelength beyond $1549 \mathrm{~nm}$. The longest wavelength that was previously evaluated is $2.4 \mu \mathrm{m}$ [66], which corresponds to a three-photon process. As the lattice absorption in silicon is limited until a wavelength of $6 \mu \mathrm{m}$ [94], it is possible to employ even longer wavelengths. However, at wavelengths of 1.5 to $6 \mu \mathrm{m}$, the Kerr coefficient of silicon is close to or above its value at $1549 \mathrm{~nm}$ [90]. Therefore, self-focussing due to the Kerr effect may restrict the usability of long wavelengths, as high instantaneous laser powers are required to induce higher-order nonlinear absorption. Moreover, the spot size will increase at longer wavelengths, which has a negative effect on the confinement of the laser energy absorption.

Solution (3) is based on the use of ultra-short pulses that, as given by the Fourier transform, have a relatively large bandwidth. By making different wavelengths follow different paths through the material towards the focus, the pulse duration outside the focus will be increased compared with the duration in the focal spot [173, 174].

\section{Improve the accuracy and capabilities of the numerical model}

To improve the numerical model, it is recommended to enhance the availability of reliable material data under conditions of high temperatures and carrier densities. An approach to address this issue could be to first perform sensitivity studies concerning the influence of inaccuracies in the material data. Secondly, methods to accurately measure the parameters that were found to have the largest influence on the model outcome could be developed. Based on a comparison of simulations with experimental data on the shape of subsurface modifications, the largest errors in the material properties are expected to be present in the data that affect the results for pulses of several hundred nanoseconds.

Improvements to the beam propagation model could also be considered. Within the present capabilities of computer systems, a rigorous solution of Maxwell's equations on a nanosecond scale, coupled with a model for the behaviour of the material, is a formidable task. It is however worthwhile to investigate the use of a unidirectional propagation model that does not require any assumptions regarding the angles of the rays with the optical axis. This would be especially beneficial to the modelling of the behaviour of the beam at the outer edge of the liquid volume inside the wafer, due to the high gradients in the optical properties that arise in this area. Moreover, it is of interest to simulate the effect of temporal focussing of the beam, which is discussed above as one of the solutions to improve the usability of ultra-short pulses. To include this phenomenon, it is necessary to remove the assumption of monochromaticity from the propagation model. 
Finally, the results of the two-temperature model could be coupled to a model to simulate the crystal growth during the resolidification of molten silicon, to assess the feasibility of predicting the exact crystal structure of the subsurface modifications.

\section{Extend the capabilities of the experimental set-up}

Experiments have so far been performed on thin wafers with a thickness of $160 \mu \mathrm{m}$. Since the performance of the processing strategies based on wavelengths of 1061 and $1549 \mathrm{~nm}$ is expected to differ for modifications at a depth of several hundred micrometres below the surface, it is of interest to investigate larger focus depths. To prevent spherical aberrations when focussing deep inside silicon, it is recommended to equip the experimental set-up with a microscope objective that contains a variable coverslip correction, preferably with some form of automated control.

Moreover, it is desirable to increase the degree of automation of the set-up, e.g. concerning the alignment of the samples, to be able to efficiently process large numbers of samples. This would enable the die strength and separation force to be reliably measured, as these quantities are stochastically distributed.

Finally, real-time measurements of the subsurface modification process are of interest. Measurements during the process can provide additional data to assess the correctness of the simulations of various physical phenomena, including the extent of the subsurface melt. A method for real-time measurements is to employ a second laser beam to probe the process, e.g. to measure the refractive index distribution inside the material by infrared interferometry [62].

\section{Additional research on analysis techniques}

Uncertainties exist in the results of the destructive analysis methods, as the possibility that the destructive sample preparation affected the measurement results cannot be ruled out. Therefore, further research on non-destructive analysis techniques is recommended.

So far, only infrared microscopy was found to be capable of detecting the presence of subsurface modifications in a non-destructive manner. However, no detailed information about the geometry of the samples could be extracted using infrared light. Possible improvements to the infrared microscopy include using coherent instead of incoherent light and applying confocal microscopy instead of regular optical microscopy.

Additionally, since a CT-scan was not capable of detecting subsurface modifications in silicon, it is worthwhile to investigate the use of a synchrotron. Synchrotron radiation allows for the three-dimensional analysis of the properties of silicon with high resolution [179].

Finally, the transmission electron microscopy results indicated that molten material resolidifies into both undamaged and damaged crystalline silicon. This observation leads to two recommendations for future research. First, for comparison with numerical modelling, it would be beneficial to identify the exact extent of the molten volume. As crystalline silicon without defects cannot be distinguished from the base material, this information could not be extracted from the transmission electron micrographs. A potential solution to this issue is to create laser modifications inside highly doped wafers, as the analysis of the transport of the dopant might enable the molten zone to be identified. However, it is necessary to ensure that the free carrier absorption as a result of ionized impurities does not prevent the successful fabrication of subsurface modifications. Secondly, it is 
recommended to investigate the exact types of lattice defects that are present, to establish their effect on the material properties of the modifications.

\section{Investigate applications beyond wafer dicing}

It is of interest to study the suitability of subsurface modifications in silicon for other applications than wafer dicing. The use of laser-induced subsurface modifications in silicon for optical applications has been proposed [64,66]. A first step in this direction would be to identify the optical properties of subsurface modifications, including the changes to the refractive index.

Apart from optical applications, laser modifications have shown to be capable of reducing the resistivity of polycrystalline silicon covered with dielectric material [180, 181]. Therefore, it is worthwhile to investigate the electrical properties of tracks of laser-induced subsurface modifications, to find out whether resistivity changes can also be induced inside (poly)silicon.

Finally, it is recommended to investigate the sensitivity of the laser modifications to specific etchants. If the response of the laser-induced modifications is different compared with the unmodified material, this implies that subsurface modifications in crystalline silicon could be applied for selective etching.

For applications in which exact control over the optical or electrical properties of subsurface modifications is required, the repeatability of the process is important. However, different crystal structures of modifications were found despite the use of identical processing conditions. Therefore, it is recommended to investigate the causes of the observed variations in the crystal structure and develop measures to eliminate these causes. 


\section{References}

[1] T. Maiman. Stimulated optical radiation in ruby. Nature, 187(4736):493-494, 1960.

[2] W. M. Steen and J. Mazumder. Laser Material Processing. Springer-Verlag, 4th edition, 2010.

[3] D. Bäuerle. Laser Processing and Chemistry. Springer-Verlag, 4th edition, 2011.

[4] R. R. Gattass and E. Mazur. Femtosecond laser micromachining in transparent materials. Nature Photonics, 2(4):219-225, 2008.

[5] J. Qiu, K. Miura, and K. Hirao. Femtosecond laser-induced microfeatures in glasses and their applications. Journal of Non-Crystalline Solids, 354:1100-1111, 2008.

[6] N. M. Bulgakova, R. Stoian, and A. Rosenfeld. Laser-induced modification of transparent crystals and glasses. Quantum Electronics, 40(11):966-985, 2010.

[7] E. G. Gamaly and A. V. Rode. Physics of ultra-short laser interaction with matter: From phonon excitation to ultimate transformations. Progress in Quantum Electronics, 37(5):215-323, 2013.

[8] S. Juodkazis, K. Nishimura, S. Tanaka, H. Misawa, E. G. Gamaly, B. Luther-Davies, L. Hallo, P. Nicolai, and V. T. Tikhonchuk. Laser-induced microexplosion confined in the bulk of a sapphire crystal: Evidence of multimegabar pressures. Physical Review Letters, 96(16):166101, 2006.

[9] E. N. Glezer and E. Mazur. Ultrafast-laser driven micro-explosions in transparent materials. Applied Physics Letters, 71(7):882-884, 1997.

[10] E. G. Gamaly, S. Juodkazis, K. Nishimura, H. Misawa, B. Luther-Davies, L. Hallo, P. Nicolai, and V. T. Tikhonchuk. Laser-matter interaction in the bulk of a transparent solid: Confined microexplosion and void formation. Physical Review B, 73(21):214101, 2006.

[11] K. Miura, J. Qiu, H. Inouye, T. Mitsuyu, and K. Hirao. Photowritten optical waveguides in various glasses with ultrashort pulse laser. Applied Physics Letters, 71(23):3329-3331, 1997.

[12] H.-B. Sun, Y. Xu, S. Juodkazis, K. Sun, M. Watanabe, S. Matsuo, H. Misawa, and J. Nishii. Arbitrary-lattice photonic crystals created by multiphoton microfabrication. Optics Letters, 26(6):325-327, 2001. 
[13] Y. Kondo, K. Nouchi, T. Mitsuyu, M. Watanabe, P. G. Kazansky, and K. Hirao. Fabrication of long-period fiber gratings by focused irradiation of infrared femtosecond laser pulses. Optics Letters, 24(10):646-648, 1999.

[14] Y. Cheng, K. Sugioka, K. Midorikawa, M. Masuda, K. Toyoda, M. Kawachi, and K. Shihoyama. Three-dimensional micro-optical components embedded in photosensitive glass by a femtosecond laser. Optics Letters, 28(13):1144-1146, 2003.

[15] Y. Cheng, H. L. Tsai, K. Sugioka, and K. Midorikawa. Fabrication of 3D microoptical lenses in photosensitive glass using femtosecond laser micromachining. Applied Physics A: Materials Science \& Processing, 85(1):11-14, 2006.

[16] Y. Hanada, K. Sugioka, I. Shihira-Ishikawa, H. Kawano, A. Miyawaki, and K. Midorikawa. 3D microfluidic chips with integrated functional microelements fabricated by a femtosecond laser for studying the gliding mechanism of cyanobacteria. Lab on a Chip, 11(12):2109-2115, 2011.

[17] E. Glezer, M. Milosavljevic, L. Huang, R. Finlay, T.-H. Her, J. Callan, and E. Mazur. Three-dimensional optical storage inside transparent materials. Optics Letters, 21(24):2023-2025, 1996.

[18] W. W. Hansen, S. W. Janson, and H. Helvajian. Direct-write UV-laser microfabrication of 3D structures in lithium-aluminosilicate glass. In Proceedings of SPIE, volume 2991, pages 104-112, 1997.

[19] S. Matsuo, S. Kiyama, Y. Shichijo, T. Tomita, S. Hashimoto, Y. Hosokawa, and H. Masuhara. Laser microfabrication and rotation of ship-in-a-bottle optical rotators. Applied Physics Letters, 93(5):051107, 2008.

[20] M. Masuda, K. Sugioka, Y. Cheng, T. Hongo, K. Shihoyama, H. Takai, I. Miyamoto, and K. Midorikawa. Direct fabrication of freely movable microplate inside photosensitive glass by femtosecond laser for lab-on-chip application. Applied Physics A: Materials Science \& Processing, 78(7):1029-1032, 2004.

[21] K. Sugioka, T. Hongo, H. Takai, and K. Midorikawa. Selective metallization of internal walls of hollow structures inside glass using femtosecond laser. Applied Physics Letters, 86(17):171910, 2005.

[22] S. Maruo, O. Nakamura, and S. Kawata. Three-dimensional microfabrication with two-photon-absorbed photopolymerization. Optics Letters, 22(2):132-134, 1997.

[23] M. Mirkhalaf, A. K. Dastjerdi, and F. Barthelat. Overcoming the brittleness of glass through bio-inspiration and micro-architecture. Nature Communications, 5:3166, 2014.

[24] L. Rapp, B. Haberl, J. E. Bradby, E. G. Gamaly, J. S. Williams, and A. V. Rode. Confined micro-explosion induced by ultrashort laser pulse at SiO2/Si interface. Applied Physics A: Materials Science \& Processing, 114(1):33-43, 2014. 
[25] P. Gise and R. Blanchard. Modern semiconductor fabrication technology. PrenticeHall, 1986.

[26] J. Czochralski. Ein neues verfahren zur messung der kristallisationsgeschwindigkeit der metalle. Zeitschrift für Physikalische Chemie, 92:219-221, 1918.

[27] W.-S. Lei, A. Kumar, and R. Yalamanchili. Die singulation technologies for advanced packaging: A critical review. Journal of Vacuum Science and Technology B, 30(4):040801, 2012.

[28] L. T. Beng, C. L. W. Yew, K. W. Shi, S. C. Teck, and K. Y. Yow. Laser grooving process development for low-k / ultra low-k devices. In Electronic Manufacturing Technology Symposium (IEMT), 2008 33rd IEEE/CPMT International, pages 1-6, 2008.

[29] J. N. Burghartz, W. Appel, H. D. Rempp, and M. Zimmermann. A new fabrication and assembly process for ultrathin chips. IEEE Transactions on Electron Devices, 56(2):321-327, 2009.

[30] B. Black, M. Annavaram, N. Brekelbaum, J. DeVale, L. Jiang, G. H. Loh, D. McCauley, P. Morrow, D. W. Nelson, D. Pantuso, P. Reed, J. Rupley, S. Shankar, J. Shen, and C. Webb. Die stacking (3D) microarchitecture. In The 39th Annual IEEE/ACM International Symposium on Microarchitecture, pages 469-479, 2006.

[31] J. van Borkulo, R. Evertsen, and R. Hendriks. Enabling technology in thin wafer dicing. In ECS Transactions, volume 18, pages 837-842, 2009.

[32] R. Fox. Dicing improvements: Yield enhancement, throughput increase and die size reduction in M/A-COM's GaAs fab. In Digest of Papers, GaAs Mantech Conference, May 21-24, Las Vegas, pages 48-51, 2001.

[33] R. Bogue. The fast-moving world of MEMS technology. Assembly Automation, 29(4):313-320, 2009.

[34] R. Albalak. Dicing MEMS handling with care. Advanced Packaging, 16(5):20-22, 2007.

[35] Y. Izawa, S. Tanaka, H. Kikuchi, Y. Tsurumi, N. Miyanaga, M. Esashi, and M. Fujita. Debris-free in-air laser dicing for multi-layer MEMS by perforated internal transformation and thermally-induced crack propagation. In IEEE 21st International Conference on Micro Electro Mechanical Systems, 2008.

[36] W. Weibull. A statistical distribution function of wide applicability. Journal of Applied Mechanics, 18(3):293-297, 1951.

[37] M. Cooke. Scribe and dice. III-Vs Review, 19(4):20-24, 2006.

[38] H. H. Gatzen. Dicing challenges in microelectronics and micro electro-mechanical systems (MEMS). Microsystem Technologies, 7(4):151-154, 2001. 
[39] K. Venkatakrishnan, N. Sudani, and B. Tan. A high-repetition-rate femtosecond laser for thin silicon wafer dicing. Journal of Micromechanics and Microengineering, 18:075032, 2008.

[40] O. Haupt, F. Siegel, A. Schoonderbeek, L. Richter, R. Kling, and A. Ostendorf. Laser dicing of silicon: Comparison of ablation mechanisms with a novel technology of thermally induced stress. Journal of Laser Micro/Nanoengineering, 3:135-140, 2008.

[41] N. Sudani, K. Venkatakrishnan, and B. Tan. Laser singulation of thin wafer: Die strength and surface roughness analysis of $80 \mu \mathrm{m}$ silicon dice. Optics and Lasers in Engineering, 47:850-854, 2009.

[42] D. Perrottet, S. Green, and B. Richerzhagen. Clean dicing of compound semiconductors using the water-jet guided laser technology. In 2006 IEEE/SEMI Advanced Semiconductor Manufacturing Conference, pages 233-236, 2006.

[43] H.-U. Zühlke, G. Eberhardt, and R. Ullmann. TLS-dicing - an innovative alternative to known technologies. In SEMI/IEEE Advanced Semiconductor Manufacturing Conference 2009, pages 28-32, 2009.

[44] F. Yoshimaro, F. Fumitsugo, F. Kenshi, and U. Naoki. Method for dicing substrate. Patent number EP1494271, Applicant: Hamamatsu Photonics K.K., 2005.

[45] F. Kenshi, F. Fumitsugu, and U. Naoki. Laser beam machining method. Patent number EP1610364, Applicant: Hamamatsu Photonics K.K., 2005.

[46] K. Fukumitsu. Laser beam machining method, laser beam machining apparatus and laser machined product. Patent number EP1649965, Applicant: Hamamatsu Photonics K.K., 2006.

[47] F. Fumitsugu, F. Kenshi, U. Naoki, and W. Toshimitsu. Laser processing apparatus with controller for positioning the focus point at different levels in the object to be processed. Patent number EP2204255, Applicant: Hamamatsu Photonics K.K., 2010.

[48] F. Fumisugo and F. Kenshi. Method of cutting object to be processed. Patent number EP2216128, Applicant: Hamamatsu Photonics K.K., 2010.

[49] K. Koji, M. Kenichi, A. Kazuhiro, and O. Tetsuya. Laser material processing system. Patent number EP2223770, Applicant: Hamamatsu Photonics K.K., 2010.

[50] A. Yoshiyuki and M. Chuichi. Semiconductor manufacturing method. Patent number US2007066044, Applicant: Renesas Technology Corporation, 2007.

[51] A. Yoshiyuki, M. Chuichi, M. Hideo, and H. Tomoko. Semiconductor device manufacturing method and semiconductor. Patent number US2009121337, Applicant: Renesas Electronics Corporation, 2009. 
[52] F. Yoshimaro, F. Fumitsugu, F. Kenshi, and U. Naoki. Substrate dividing method. Patent number EP1632997, Applicant: Hamamatsu Photonics K.K., 2006.

[53] H. Keiichi and N. Yuuki. Method for producing semiconductor chip with adhesive film, adhsive film for semiconductor used in the method, and method for producing semiconductor device. Patent number EP2200075, Applicant: Hitachi Chemical Company, Ltd., 2010.

[54] E. Ohmura, F. Fukuyo, K. Fukumitsu, and H. Morita. Internal modified-layer formation mechanism into silicon with nanosecond laser. Journal of Achievements in Materials and Manufacturing Engineering, 17:381-384, 2006.

[55] E. Ohmura, K. Ogawa, M. Kumagai, M. Nakano, K. Fukumitsu, and H. Morita. Analysis of crack propagation in stealth dicing using stress intesity factor. In Proceedings of LAMP2009 - the 5th International Congress on Laser Advanced Materials Processing, 2009.

[56] M. Kumagai, N. Uchiyama, E. Ohmura, R. Sugiura, K. Atsumi, and K. Fukumitsu. Advanced dicing technology for semiconductor wafer-stealth dicing. IEEE Transactions on Semiconductor Manufacturing, 20(3):259-265, 2007.

[57] M. Birkholz, K.-E. Ehwald, M. Kaynak, T. Semperowitsch, B. Holz, and S. Nordhoff. Separation of extremely miniaturized medical sensors by IR laser dicing. Journal of Optoelectronics and Advanced Materials, 12:479-483, 2010.

[58] M. Kumagai, T. Sakamoto, and E. Ohmura. Laser processing of doped silicon wafer by the stealth dicing. In International Symposium on Semiconductor Manufacturing 2007, 2007.

[59] C. Li, X. Shi, J. Si, F. Chen, T. Chen, Y. Zhang, and X. Hou. Photoinduced multiple microchannels inside silicon produced by a femtosecond laser. Applied Physics B: Lasers and Optics, 98(2-3):377-381, 2010.

[60] T. Chen, J. Si, X. Hou, S. Kanehira, K. Miura, and K. Hirao. Photoinduced microchannels inside silicon by femtosecond pulses. Applied Physics Letters, 93(5):051112, 2008.

[61] E. Ohmura. Temperature rise of silicon due to absorption of permeable pulse laser. In V. S. Vikhrenko, editor, Heat Transfer - Engineering Applications, chapter 2. InTech, 2011.

[62] V. V. Kononenko, V. V. Konov, and E. M. Dianov. Delocalization of femtosecond radiation in silicon. Optics Letters, 37(16):3369-3371, 2012.

[63] S. Leyder, D. Grojo, P. Delaporte, W. Marine, M. Sentis, and O. Utéza. Multiphoton absorption of $1.3 \mu \mathrm{m}$ wavelength femtosecond laser pulses focused inside Si and $\mathrm{SiO}_{2}$. In Proceedings of SPIE, volume 8770, paper 877004, 2013. 
[64] V. V. Parsi Sreenivas, M. Bülters, and R. B. Bergmann. Microsized subsurface modification of mono-crystalline silicon via non-linear absorption. Journal of the European Optical Society - Rapid publications, 7:12035, 2012.

[65] Y. Ito, H. Sakashita, R. Suzuki, M. Uewada, K. P. Luong, and R. Tanabe. Modification and machining on back surface of a silicon substrate by femtosecond laser pulses at $1552 \mathrm{~nm}$. Journal of Laser Micro/Nanoengineering, 9(2):98-102, 2014.

[66] A. H. Nejadmalayeri, P. R. Herman, J. Burghoff, M. Will, S. Nolte, and A. Tünnermann. Inscription of optical waveguides in crystalline silicon by mid-infrared femtosecond laser pulses. Optics Letters, 30(9):964-966, 2005.

[67] G. E. Jellison, Jr. and D. H. Lowndes. Optical absorption coefficient of silicon at $1.152 \mu \mathrm{m}$ at elevated temperatures. Applied Physics Letters, 41(7):594-596, 1982.

[68] B. Dillingh. Laser-matter interaction modelling for application in the ALISS-process. Technical report, TNO, 2010.

[69] I. B. Bogatyrev, D. Grojo, P. Delaporte, S. Leyder, M. Sentis, W. Marine, and T. E. Itina. Non-linear absorption of 1.3- $\mu \mathrm{m}$ wavelength femtosecond laser pulses focused inside semiconductors: Finite difference time domain-two temperature model combined computational study. Journal of Applied Physics, 110(10):103106, 2011.

[70] K. Yee. Numerical solution of initial boundary value problems involving Maxwell's equations in isotropic media. IEEE Transactions on Antennas and Propagation, 14(3):302-307, 1966.

[71] E. V. Zavedeev, V. V. Kononenko, V. M. Gololobov, and V. I. Konov. Modeling the effect of fs light delocalization in Si bulk. Laser Physics Letters, 11(3):036002, 2014.

[72] H. M. van Driel. Kinetics of high-density plasmas generated in Si by 1.06- and 0.53- $\mu \mathrm{m}$ picosecond laser pulses. Physical Review B, 35(15):8166-8176, 1987.

[73] A. L. Smirl, I. W. Boyd, T. F. Boggess, S. C. Moss, and H. M. van Driel. Structural changes produced in silicon by intense 1- $\mathrm{mm}$ ps pulses. Journal of Applied Physics, 60(3):1169-1182, 1986.

[74] A. Lietoila and J. F. Gibbons. Computer modeling of the temperature rise and carrier concentration induced in silicon by nanosecond laser pulses. Journal of Applied Physics, 53(4):3207-3213, 1982.

[75] J. Chen, D. Tzou, and J. Beraun. Numerical investigation of ultrashort laser damage in semiconductors. International Journal of Heat and Mass Transfer, 48(3-4):501509, 2005.

[76] C. Ma, W.-Y. Ho, R. M. Walser, and M. F. Becker. Transient laser-induced surface deformation of silicon in relation to damage. In Proceedings of SPIE, volume 1848, pages 59-71, 1993. 
[77] C. Kittel. Introduction to Solid State Physics. John Wiley \& Sons, 8th edition, 2005.

[78] R. Hull, editor. Properties of Crystalline Silicon. The Institution of Electrical Engineering, 1999.

[79] T. Morishita. High density amorphous form and polyamorphic transformations of silicon. Physical Review Letters, 93(5):055503, 2004.

[80] V. Domnich and Y. Gogotsi. Phase transformations in silicon under contact loading. Reviews on Advanced Materials Science, 3:1-36, 2002.

[81] P. Pirouz, R. Chaim, U. Dahmen, and K. H. Westmacott. The martensitic transformation in silicon-I experimental observations. Acta Materialia, 38(2):313-322, 1990.

[82] M. Hanfland, U. Schwarz, K. Syassen, and K. Takemura. Crystal structure of the high-pressure phase silicon VI. Physical Review Letters, 82(6):1197-1200, 1999.

[83] D. Ge, V. Domnich, and Y. Gogotsi. Thermal stability of metastable silicon phases produced by nanoindentation. Journal of Applied Physics, 95(5):2725-2731, 2004.

[84] A. K. McMahan and J. A. Moriarty. Structural phase stability in third-period simple metals. Physical Review B, 27(6):3235-3251, 1983.

[85] B. E. A. Saleh and M. C. Teich. Fundamentals of Photonics. John Wiley \& Sons, second edition, 2007.

[86] A. Einstein. Über einen die erzeugung und verwandlung des lichtes betreffenden heuristischen gesichtspunkt. Annalen der Physik, 322(6):132-148, 1905.

[87] H. H. Li. Refractive index of silicon and germanium and its wavelength and temperature derivatives. Journal of Physical and Chemical Reference Data, 9(3):561-658, 1980.

[88] A. D. Bristow, N. Rotenberg, and H. M. van Driel. Two-photon absorption and Kerr coefficients of silicon for 850-2200 nm. Applied Physics Letters, 90(19):191104, 2007.

[89] G. Fibich and A. L. Gaeta. Critical power for self-focusing in bulk media and in hollow waveguides. Optics Letters, 25(5):335-337, 2000.

[90] T. Wang, N. Venkatram, J. Gosciniak, Y. Cui, G. Qian, W. Ji, and D. T. H. Tan. Multi-photon absorption and third-order nonlinearity in silicon at mid-infrared wavelengths. Optics Express, 21(26):32192-32198, 2013.

[91] A. Singh. Free charge carrier induced refractive index modulation of crystalline silicon. In IEEE International Conference on Group IV Photonics, pages 102-104, 2010 . 
[92] S. M. Sze and K. K. Ng. Physics of Semiconductor Devices. John Wiley \& Sons, third edition, 2007.

[93] Y. P. Varshni. Temperature dependence of the energy gap in semiconductors. Physica, 34(1):149-154, 1967.

[94] J. E. Peters, P. D. Ownby, C. R. Poznich, J. C. Richter, and D. W. Thomas. Infrared absorption of Czochralski germanium and silicon. In Proceedings of SPIE, volume 4452, pages 17-24, 2001.

[95] M. V. Ammosov, N. B. Delone, and V. P. Krainov. Tunnel ionization of complex atoms and atomic ions in electromagnetic field. Soviet Physics, JETP, 64:1191-1194, 1986.

[96] D. Grojo, S. Leyder, P. Delaporte, W. Marine, M. Sentis, and O. Utéza. Longwavelength multiphoton ionization inside band-gap solids. Physical Review B, 88(19):195135, 2013.

[97] S. K. Sundaram and E. Mazur. Inducing and probing non-thermal transitions in semiconductors using femtosecond laser pulses. Nature Materials, 1(4):217-224, 2002.

[98] J. Jia, M. Li, and C. V. Thompson. Amorphization of silicon by femtosecond laser pulses. Applied Physics Letters, 84(16):3205-3207, 2004.

[99] M. Amer, M. El-Ashry, L. Dosser, K. Hix, J. Maguire, and B. Irwin. Femtosecond versus nanosecond laser machining: comparison of induced stresses and structural changes in silicon wafers. Applied Surface Science, 242(1-2):162-167, 2005.

[100] A. L. Smirl, T. F. Boggess, S. C. Moss, and I. W. Boyd. Pulsewidth-dependence of nonlinear energy deposition and redistribution in Si, GaAs and Ge during $1 \mu \mathrm{m}$ picosecond irradiation. Journal of Luminescence, 30(1-4):272-289, 1985.

[101] K. Yamaguchi and K. Itagaki. Measurement of high temperature heat content of silicon by drop calorimetry. Journal of Thermal Analysis and Calorimetry, 69(3):10591066, 2002.

[102] A. G. Cullis, N. G. Chew, H. C. Webber, and D. J. Smith. Orientation dependence of high speed silicon crystal growth from the melt. Journal of Crystal Growth, 68(2):624-638, 1984.

[103] K. E. Petersen. Silicon as a mechanical material. Proceedings of the IEEE, 70(5):420457, 1982.

[104] M. Brede. The brittle-to-ductile transition in silicon. Acta Materialia, 41(1):211228, 1993.

[105] P. Stampfli and K. H. Bennemann. Theory for the instability of the diamond structure of Si, Ge, and C induced by a dense electron-hole plasma. Physical Review B, 42(11):7163-7173, 1990. 
[106] J.-T. Wang, C. Chen, H. Mizuseki, and Y. Kawazoe. Kinetic origin of divergent decompression pathways in silicon and germanium. Physical Review Letters, 110(16):165503, 2013.

[107] G. Cerullo and S. De Silvestri. Ultrafast optical parametric amplifiers. Review of Scientific Instruments, 74(1):1-18, 2003.

[108] G. Keiser. Optical Fiber Communications. McGraw-Hill, 4th edition, 2011.

[109] M. I. Kaganov, I. M. Livshitz, and L. V. Tanatarov. Relaxation between electrons and the crystalline lattice. Soviet Physics, JETP, 4:173-178, 1957.

[110] F. Berz, R. W. Cooper, and S. Fagg. Recombination in the end regions of pin diodes. Solid-State Electronics, 22(3):293-301, 1979.

[111] A. Rousse, C. Rischel, S. Fourmaux, I. Uschmann, S. Sebban, G. Grillon, P. Balcou, E. Förster, J. P. Geindre, P. Audebert, J. C. Gauthier, and D. Hulin. Non-thermal melting in semiconductors measured at femtosecond resolution. Nature, 410(6824):6567, 2001.

[112] C. Bonacina, G. Comini, A. Fasano, and M. Primicerio. Numerical solution of phasechange problems. International Journal of Heat and Mass Transfer, 16(10):18251832, 1973.

[113] H. R. Shanks, P. D. Maycock, P. H. Sidles, and G. C. Danielson. Thermal conductivity of silicon from 300 to $1400^{\circ}$ K. Physical Review, 130(5):1743-1748, 1963.

[114] N. G. Nilsson. Band-to-band Auger recombination in silicon and germanium. Physica Scripta, 8(4):165-176, 1973.

[115] W. van Roosbroeck and W. Shockley. Photon-radiative recombination of electrons and holes in germanium. Physical Review, 94(6):1558-1560, 1954.

[116] W. Shockley and W. T. Read, Jr. Statistics of the recombinations of holes and electrons. Physical Review, 87(5):835-842, 1952.

[117] C. G. B. Garrett and W. H. Brattain. Physical theory of semiconductor surfaces. Physical Review, 99(2):376-387, 1955.

[118] R. D. Cook, D. S. Malkus, M. E. Plesha, and R. J. Witt. Concepts and Applications of Finite Element Analysis. John Wiley \& Sons, 2002.

[119] P. Varga and P. Török. The Gaussian wave solution of Maxwell's equations and the validity of scalar wave approximation. Optics Communications, 152(1-3):108-118, 1998.

[120] E. Wolf. A scalar representation of electromagnetic fields: II. Proceedings of the Physical Society, 74(3):269-280, 1959. 
[121] A. C. Newell and J. V. Moloney. Nonlinear Optics. Addison-Wesley Publishing Company, 1992.

[122] A. Couairon, E. Brambilla, T. Corti, D. Majus, O. de J. Ramírez-Góngora, and M. Kolesik. Practitioner's guide to laser pulse propagation models and simulation. The European Physical Journal - Special Topics, 199:5-76, 2011.

[123] R. Temam and A. Miranville. Mathematical Modeling in Continuum Mechanics. Cambridge University Press, 2nd edition, 2005.

[124] S. A. Collins, Jr. Lens-system diffraction integral written in terms of matrix optics. Journal of the Optical Society of America, 60(9):1168-1177, 1970.

[125] L. Vicari and F. Bloisi. Matrix representation of axisymmetric optical systems including spatial filters. Applied Optics, 28(21):4682-4686, 1989.

[126] A. E. Siegman. Lasers. University Science Books, 1986.

[127] A. E. Siegman. How to (maybe) measure laser beam quality. In DPSS (Diode Pumped Solid State) Lasers: Applications and Issues, paper MQ1. Optical Society of America, 1998.

[128] MSC Software Corporation. Marc 2008 r1. Volume A: Theory and User Information, 2008.

[129] S.-C. Sheng and A. E. Siegman. URL https : / / web . archive . org / web / 20080813082836/http://www. stanford . edu/ siegman/sheng_fht_package txt. URL refers to a permanent archive containing a copy retrieved 13-8-2008.

[130] ISO/IEC. 1539-1:2010: Information technology - programming languages - Fortran - part 1: Base language. Technical report, International Organization for Standardization, 2010.

[131] D. S. Kershaw. The incomplete Cholesky-conjugate gradient method for the iterative solution of systems of linear equations. Journal of Computational Physics, 26(1):43-65, 1978.

[132] K. H. Huebner, E. A. Thornton, and T. G. Byrom. The Finite Element Method for Engineers. John Wiley \& Sons, 1995.

[133] MSC. Software. Marc 120 - Section 16 - Heat Transfer Analysis, 2001.

[134] A. E. Siegman. Quasi fast Hankel transform. Optics Letters, 1(1):13-15, 1977.

[135] Intel Corporation. Intel® Math Kernel Library 10.3 Update 8 Reference Manual, 2011.

[136] A. Agnesi, G. C. Reali, G. Patrini, and A. Tomaselli. Numerical evaluation of the Hankel transform: remarks. Journal of the Optical Society of America A, Optics and Image Science, 10(9):1872-1874, 1993. 
[137] R. J. LeVeque. Finite Difference Methods for Ordinary and Partial Differential Equations. Society for Industrial and Applied Mathematics, Philadelphia, 2007.

[138] P. D. Lax and R. D. Richtmyer. Survey of the stability of linear finite difference equations. Communications on Pure and Applied Mathematics, 9(2):267-293, 1956.

[139] M. Özişik. Boundary Value Problems of Heat Conduction. Dover Publications, 2002.

[140] J. Crank and E. Nicolson. A practical method for numerical evaluation of solutions of partial differential equations of the heat-conduction type. Proceedings of the Cambridge Philosophical Society, 43:50-67, 1947.

[141] E. Anderson, Z. Bai, C. Bischof, S. Blackford, J. Demmel, J. Dongarra, J. Du Croz, A. Greenbaum, S. Hammarling, A. McKenney, and D. Sorensen. LAPACK Users' Guide. Society for Industrial and Applied Mathematics, Philadelphia, PA, third edition, 1999.

[142] F. Bashforth and J. C. Adams. An Attempt to test the Theories of Capillary Action by comparing the theoretical and measured forms of drops of fluid. With an explanation of the method of integration employed in constructing the tables which give the theoretical forms of such drops. Cambridge University Press, 1883.

[143] Y. Chung and N. Dagli. Analysis of z-invariant and z-variant semiconductor rib waveguides by explicit finite difference beam propagation method with nonuniform mesh configuration. IEEE Journal of Quantum Electronics, 27(10):2296 -2305, 1991.

[144] D. R. Durran. The third-order Adams-Bashforth method: an attractive alternative to leapfrog time differencing. Monthly Weather Review, 119(3):702-720, 1991.

[145] R. Asselin. Frequency filter for time integrations. Monthly Weather Review, 100(6):487-490, 1972.

[146] R. E. Hummel. Electronic Properties of Materials. Springer-Verlag, 3rd edition, 2001.

[147] T. Sjodin, H. Petek, and H.-L. Dai. Ultrafast carrier dynamics in silicon: A twocolor transient reflection grating study on a (111) surface. Physical Review Letters, 81(25):5664-5667, 1998.

[148] M. W. Chase. NIST-JANAF thermochemical tables. Journal of Physical and Chemical Reference Data Monographs \& Supplements, (9), 1998.

[149] C. J. Glassbrenner and G. A. Slack. Thermal conductivity of silicon and germanium from $3^{\circ} \mathrm{K}$ to the melting point. Physical Review, 134(4A):A1058-A1069, 1964.

[150] K. G. Svantesson and N. G. Nilsson. Determination of the temperature dependence of the free carrier and interband absorption in silicon at $1.06 \mu \mathrm{m}$. Journal of Physics C: Solid State Physics, 12(18):3837-3842, 1979. 
[151] G. E. Jellison, Jr. and F. A. Modine. Optical functions of silicon between 1.7 and $4.7 \mathrm{eV}$ at elevated temperatures. Physical Review B, 27(12):7466-7472, 1983.

[152] P. M. Fauchet and K. D. Li. The dielectric function of laser-produced molten Si. Journal of Non-Crystalline Solids, 97-98:1267-1270, 1987.

[153] P. Jonsson, H. Bleichner, M. Isberg, and E. Nordlander. The ambipolar Auger coefficient: Measured temperature dependence in electron irradiated and highly injected n-type silicon. Journal of Applied Physics, 81(5):2256-2262, 1997.

[154] J. M. Dorkel and P. Leturcq. Carrier mobilities in silicon semi-empirically related to temperature, doping and injection level. Solid-State Electronics, 24(9):821-825, 1981.

[155] Y. Okada and Y. Tokumaru. Precise determination of lattice parameter and thermal expansion coefficient of silicon between 300 and 1500 K. Journal of Applied Physics, 56(2):314-320, 1984.

[156] V. L. Spiering, S. Bouwstra, and R. M. E. J. Spiering. On-chip decoupling zone for package-stress reduction. Sensors and Actuators A: Physical, 39(2):149-156, 1993.

[157] G. B. Airy. On the diffraction of an object-glass with circular aperture. Transactions of the Cambridge Philosophical Society, 5:283-291, 1835.

[158] F. L. Pedrotti and L. S. Pedrotti. Introduction to Optics. Prentice-Hall, 1993.

[159] M. J. Nasse and J. C. Woehl. Realistic modeling of the illumination point spread function in confocal scanning optical microscopy. Journal of the Optical Society of America A, Optics and Image Science, 27(2):295-302, 2010.

[160] P. Török and P. Varga. Electromagnetic diffraction of light focused through a stratified medium. Applied Optics, 36(11):2305-2312, 1997.

[161] J. H. Marburger. Self-focusing: Theory. Progress in Quantum Electronics, 4:35-110, 1975.

[162] J. Wagner and J. A. del Alamo. Band-gap narrowing in heavily doped silicon: A comparison of optical and electrical data. Journal of Applied Physics, 63(2):425429, 1988.

[163] G. Canat, L. Lombard, S. Jetschke, S. Unger, J. Kirchhof, H.-R. Müller, A. Durécu, V. Jolivet, and P. Bourdon. Er-Yb-doped LMA fiber structures for high energy amplification of narrow linewidth pulses at $1.5 \mu \mathrm{m}$. In Conference on Lasers and Electro Optics and the Quantum Electronics and Laser Science Conference, paper CTuBB1. Optical Society of America, 2007.

[164] P. Wan, J. Liu, L.-M. Yang, and F. Amzajerdian. Low repetition rate high energy $1.5 \mu \mathrm{m}$ fiber laser. Optics Express, 19(19):18067-18071, 2011. 
[165] Y. Leng. Materials Characterization: Introduction to Microscopic and Spectroscopic Methods. John Wiley \& Sons, 2008.

[166] W. C. Dash and R. Newman. Intrinsic optical absorption in single-crystal germanium and silicon at 77K and 300K. Physical Review, 99(4):1151-1155, 1955.

[167] P. J. Phillips, M. J. Mills, and M. De Graef. STEM dislocation analysis and image simulations. Microscopy and Microanalysis, 17:912-913, 2011.

[168] I. W. Boyd, S. C. Moss, T. F. Boggess, and A. L. Smirl. Various phase transitions and changes in surface morphology of crystalline silicon induced by 4-260-ps pulses of 1- $\mu \mathrm{m}$ radiation. Applied Physics Letters, 45(1):80-82, 1984.

[169] X. Wang, Z. H. Shen, J. Lu, and X. W. Ni. Laser-induced damage threshold of silicon in millisecond, nanosecond, and picosecond regimes. Journal of Applied Physics, 108(3):033103, 2010.

[170] J. R. Meyer, M. R. Kruer, and F. J. Bartoli. Optical heating in semiconductors: Laser damage in Ge, Si, InSb, and GaAs. Journal of Applied Physics, 51(10):5513-5522, 1980.

[171] S. D. Jackson. Towards high-power mid-infrared emission from a fibre laser. Nature Photonics, 6(7):423-431, 2012.

[172] O. Henderson-Sapir, J. Munch, and D. J. Ottaway. Mid-infrared fiber lasers at and beyond $3.5 \mu \mathrm{m}$ using dual-wavelength pumping. Optics Letters, 39(3):493-496, 2014.

[173] D. Oron, E. Tal, and Y. Silberberg. Scanningless depth-resolved microscopy. Optics Express, 13(5):1468-1476, 2005.

[174] G. Zhu, J. van Howe, M. Durst, W. Zipfel, and C. Xu. Simultaneous spatial and temporal focusing of femtosecond pulses. Optics Express, 13(6):2153-2159, 2005.

[175] Y. H. Lee, R. Biswas, C. M. Soukoulis, C. Z. Wang, C. T. Chan, and K. M. Ho. Molecular-dynamics simulation of thermal conductivity in amorphous silicon. Physical Review B, 43(8):6573-6580, 1991.

[176] K. Shimamura, J. Okuma, S. Ohmura, and F. Shimojo. Molecular-dynamics study of void-formation inside silicon wafers in stealth dicing. Journal of Physics: Conference Series, 402(1):012044, 2012.

[177] C. C. Yang, J. C. Li, and Q. Jiang. Temperature-pressure phase diagram of silicon determined by Clapeyron equation. Solid State Communications, 129(7):437-441, 2004.

[178] Z. Zhou, S. Mukherjee, and W.-K. Rhim. Measurement of thermophysical properties of molten silicon using an upgraded electrostatic levitator. Journal of Crystal Growth, 257(3-4):350-358, 2003. 
[179] B. C. Larson, C. W. White, T. S. Noggle, and D. Mills. Synchrotron X-ray diffraction study of silicon during pulsed-laser annealing. Physical Review Letters, 48(5):337340, 1982.

[180] E. Boulais, J. Fantoni, A. Chateauneuf, Y. Savaria, and M. Meunier. Laser-induced resistance fine tuning of integrated polysilicon thin-film resistors. IEEE Transactions on Electron Devices, 58(2):572-575, 2011.

[181] R. Singh, Y. Audet, Y. Gagnon, Y. Savaria, E. Boulais, and M. Meunier. A lasertrimmed rail-to-rail precision CMOS operational amplifier. Transactions on Circuits and Systems Part II - Express Briefs, 58(2):75-79, 2011. 


\section{Acknowledgements}

The work that I performed during the last four years would not have been possible without the support of several people. First, I would like to thank my supervisor, Bert Huis in 't Veld and my co-supervisor, Gert-Willem Römer, for providing me the opportunity to perform this research and their support. Martina Tjapkes also deserves a thank you for her help with all the formalities. I acknowledge the members of the graduation committee, prof. dr. ir. H.W.M. Hoeijmakers, prof. dr. J.G.E. Gardeniers, prof. Dr.-Ing. habil. A. Ostendorf, prof. dr. ir. M.G.D. Geers and prof. dr. ir. A. de Boer for evaluating my thesis. Financial support was provided by Point-One and Advanced Laser Separation International N.V. (ALSI). I appreciate the advice that I received from the employees of ALSI, including Guido Knippels, Peter Chall, Jürgen Betz and Mark Müller.

My work depended on specialised equipment and analysis techniques. I am grateful to the people who made this possible. Leo Tiemersma fabricated components for my set-up. Gerald Ebberink was in charge of the maintenance of the lab and, together with Laura Vargas, assisted me with microscopy. Fred van Goor lent me a laser source. Jürgen Betz supplied the silicon base material. Lachlan Smillie of the Australian National University, with help from Jim Williams, Jodie Bradby and Bianca Haberl, has invested significant effort in analysing the material structure of my samples, using equipment from the Australian National Fabrication Facility and the Centre for Advanced Microscopy. Additional sample analyses were performed by Frank Rietveld, Harry Roberts and Xiao-Mei Zhang of NXP Semiconductors Regional Quality Center Nijmegen. Finally, Bert Dillingh of TNO Science and Industry supplied code for interfacing with finite element software.

I would also like to thank the rest of the staff of the WA group and M2i: Ronald, Dannis, Johannes, B. Pathiraj, Jaap, Wouter and Jonathan and the staff of the spin-offs: Laser Applicatie Centrum and Kite Robotics for their advice and the serious and less serious discussions during lunch and coffee breaks. Apart from discussing research, my $\mathrm{PhD}$ and Postdoc colleagues: Johann, Jüstus, Steven, Jozef, Ralph, Davide, Ni Ya, Bert, Ger, Daniël, Jitendra, Ali, Volkert and Dirk, made my time at the university enjoyable. Ralph: thank you for being my paranymph. Johann, Steven, Jozef and Satadal: thank you for being friends and all the fun we had while having dinner, drinks or watching movies at Steven's place.

Finally, a big thank you goes to my mother, father and brother for their support. 




\section{Laser-Induced Subsurface Modification of Silicon Wafers}

Wafer dicing is the technology to separate wafers into individual components known as dies. New developments in the semiconductor industry, such as die stacking and the development of microelectromechanical systems, present significant challenges to the dicing process.

A promising wafer dicing method is laser-induced subsurface separation, as it is dry and debris-free. This method consists of two steps. First, subsurface modifications are created inside the wafer by pulsed lasers, without inducing any damage to the surface. Secondly, a force is exerted on the wafer, causing it to separate along the laser-induced modifications.

The objective of this thesis is to investigate the laser-material interaction during the formation of subsurface modifications in crystalline silicon. Based on experiments and numerical simulations, laser processes are proposed that are suitable for integration in industrial equipment. 